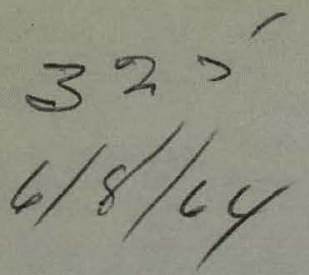

\title{
X-RAY FLUORESCENCE TABLES: LITHIUM FLUORIDE CRYSTAL
}
W. P. Amsbury
W. W. Lee
J. H. Rowan
G. E. Walden 


\section{DISCLAIMER}

This report was prepared as an account of work sponsored by an agency of the United States Government. Neither the United States Government nor any agency Thereof, nor any of their employees, makes any warranty, express or implied, or assumes any legal liability or responsibility for the accuracy, completeness, or usefulness of any information, apparatus, product, or process disclosed, or represents that its use would not infringe privately owned rights. Reference herein to any specific commercial product, process, or service by trade name, trademark, manufacturer, or otherwise does not necessarily constitute or imply its endorsement, recommendation, or favoring by the United States Government or any agency thereof. The views and opinions of authors expressed herein do not necessarily state or reflect those of the United States Government or any agency thereof. 


\section{DISCLAIMER}

Portions of this document may be illegible in electronic image products. Images are produced from the best available original document. 
Printed in USA. Price: $\$ 2.50$ Available from the

Office of Technical Services

U. S. Department of Commerce

Washington 25, D. C.

\section{LEGAL NOTICE}

This report was prepared as an account of Government sponsored work. Neither the United States, nor the Commission, nor any person acting on behalf of the Commission:

A. Makes any warranty or representation, expressed or implied, with respect to the accuracy, completeness, or usefulness of the information contained in this report, or that the use of any information, apparatus, method, or process disclosed in this report may not infringe privately owned rights; or

B. Assumes any liabilities with respect to the use of, or for damages resulting from the use of any information, apparatus, method, or process disclosed in this report.

As used in the above, "person acting on behalf of the Commission" includes any employee or contractor of the Commission, or employee of such contractor, to the extent that such employee or contractor of the Commission, or employee of such contractor prepares, disseminates, or provides access to, any information pursuant to his employment or contract with the Commission, or his employment with such contractor. 


\title{
UNION CARBIDE CORPORATION \\ Nuclear Division
}

\section{Y-12 PLANT}

$$
\text { Contract W-7405-eng-26 }
$$

With the US Atomic Energy Commission

\author{
X-RAY FLUORESCENCE TABLES: LITHIUM FLUORIDE CRYSTAL \\ W. P. Amsbury \\ W. W. Lee \\ J. H. Rowan \\ G. E. Walden
}

Oak Ridge, Tennessee

April 21, 1961 
Report Number $Y-1470-C$

Chemistry

TID-4500 (29th Edition)

Distribution

Amsbury, W. P.

Bailey, E.W.

Ballenger, $H$. F.

Barton, T. H.

Bell, B. B.

Bernander, N.K.

Briscoe, O. W.

Burkhart, L. E.

Center, C. E.

Cowen, D. D.

(ORGDP)

(ORNL)

Feldman, $C$.

(ORNL)

Googin, J. M.

Harwell, W. L.

Hemphill, L. F.

(ORGDP) (5)

Huber, A. P.

(ORGDP)

Keller, C. A:

(AEC-ORO) (4)

Kienberger, C. A. (ORGDP)

Lee, W. W.

Lewis, $F . O$.

(ORGDP)

McLendon, J. D.

Mitchel, G.W.

Patton, F.S.

Postma, F.W.

Rowan, J. H.

Stewart, J. H.

Tench, F. M.

Walden, G. E.

Weber, C. W.

Whitson, W. K.

(ORGDP)

Winkel, R. A.

$Y-12$ Central Files

(Paducah)

$Y-12$ Central Files

$(\mathrm{Y}-12 \mathrm{RC})$

In addition, this report is distributed in accordance with the category, Chemistry as given in the "USAEC Standard Distribution Lists. for Unclassified Scientific and Technical Reports", TID-4500 (29th Edition), April 1, 1964. 
ABSTRACT

A critical compilation of standard reference tables of $X$-ray fluorescence lines has been published in convenient form for experimental use. This section of the report contains tables calculated for a lithium fluoride crystal. 


\section{THIS PAGE}

WAS INTENTIONALLY

LEFT BLANK 


\section{CONTENTS}

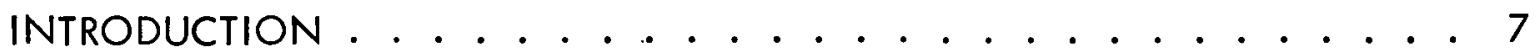

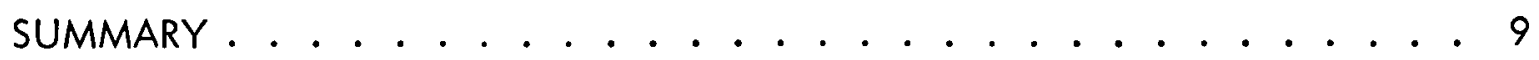

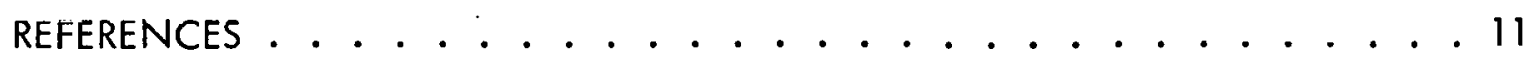

TABLE I (GONIOMETER ANGLES ARRANGED BY ELEMENT, LINE, AND ORDER)........ 13

TABLE 2 (GONIOMETER ANGLES ARRANGED BY ANGLE MAGNITUDE). . . 57 


\section{THIS PAGE}

\section{WAS INTENTIONALLY \\ LEFT BLANK}




\section{INTRODUCTION}

Standard $X$-ray fluorescence tables contain rounding errors and a few disjointed entries. Furthermore, their format is not the most convenient one in some experimental situations, leaving to the experimenter the task of hunting and comparing before coming to a definite conclusion about the composition of his sample. For a specific analyzing crystal, the experimenter needs a table that is arranged according to element, order, and line; and an angular tabulation. This section of the report provides these tables for the lithium fluoride crystal.

Wavelength data from three authors: Müller, ${ }^{(1)}$ Powers, $^{(2)}$ and Liebhafsky, ${ }^{(3)}$ were punched into cards, compared, sorted, and sifted with a computer, and a "best" value determined from the acceptable values. Goniometer angles were generated for specific analyzing crystals and the resulting angles were printed in two tables. One of the tables is arranged by element, order, and line and the other by angle magnitude. Tables may be generated for any crystal with known spacing.

The tables have been compiled for all emissions having $2 \theta$ reflections less than 150 degrees. Reflections above $150^{\circ} 2 \theta$ are not considered as practical since the reflection efficiency becomes increasingly poorer with increasing angles. Few instruments are capable of even operating in this range. The logical approach when the desired emission falls at these high angles would be the selection of an analyzing crystal with more appropriate d-spacing. Orders from 1 through 5 for elements from sodium (11) through californium (98) have been included in the tables. Data where available for the more prominent $M$ spectra have also been included.

Relative intensity data have not been included as a part of this report since such data are readily available to those who desire it; however, in some cases there are large variations in the relative values that are assigned to a given energy level. It is felt that the data of Powers (2) are satisfactory for most uses so to include such data in this report would only consume unnecessary space.

This report is being published in several sections. Each section contains both tables for a specific analyzing crystal. The first section(4) lists and explains the operation of the computer programs which have generated the tables. The reader is invited to refer to this section of the report for further information. 
THIS PAGE

\section{WAS INTENTIONALLY LEFT BLANK}


SUMMARY

Tables arranged by element, order, and line, and also angular tabulations for a specific analyzing crystal, were derived from a critical amalgamation of three standard reference tables of $X$-ray fluorescence lines. The format of these tables was designed to increase the efficiency of the experimenter by decreasing the time spent in table searches. This section of the report contains both tables for a lithium fluoride crystal. 
THIS PAGE

\section{WAS INTENTIONALLY LEFT BLANK}




\section{REFERENCES}

(1) Müller, Dr. Phil.E. A.W., Wellenlängen - und Winkeltafeln zur Rontgenspektralanalyse, Siemens and Halske Aktiengesellschaft, Karlsruhe, West Germany (1960).

(2) Powers, Maurice C., X-Ray Fluorescent Spectrometer Conversion Tables for Topaz, LiF, NaCl, EDDT, and ADP Crystals, Phillips Electronics, Inc, Instruments Division, Mount Vernon, New York (1957).

(3) Liebhafsky, H. A., Pfeiffer, H. A., Winslow, E. W., Zemany, P. D., X-Ray Absorption and Emission in Analytical Chemistry, John Wiley and Sons, Inc, New York (1960).

(4) Amsbury, W. P., Lee, W. W., Rowan, J. H., Walden, G. E., X-Ray Fluorescence Tables: Program Description, $Y-1470-A$, Union Carbide Corporation, Nuclear Division, Y-12 Plant, Oak Ridge, Tennessee (to be issued). 
THIS PAGE

\section{WAS INTENTIONALLY LEFT BLANK}


TABLE 1

GONIOMETER ANGLES ARRANGED BY ELEMENT, LINE, AND ORDER 
THIS PAGE

\section{WAS INTENTIONALLY LEFT BLANK}




\begin{tabular}{|c|c|c|c|c|c|c|c|c|c|c|c|}
\hline 1) & $\mathrm{CL}$ & & & LITHIUM & FLIJORIDE CRYSTAL, & D\# 2.014 & ANGSTROMS & & & & \\
\hline & $\begin{array}{c}\text { ORDER } \\
1 \\
2 \\
3 \\
4 \\
5\end{array}$ & KAI & KA2 & $K B I$ & KB2 & $\mathrm{KB3}$ & KB 4 & KBS & LAI & LA2 & LBI \\
\hline & $\begin{array}{l}1 \\
2 \\
3 \\
4 \\
5\end{array}$ & LB2 & LB3 & L84 & L85 & LB6 & LB7 & L89 & LBIO & $|Y|$ & LY2 \\
\hline & $\begin{array}{l}1 \\
2 \\
3 \\
4 \\
5\end{array}$ & L.Y3 & LY4 & LYS & LYo & LN & LL & MAI & MA2 & MB & MY \\
\hline & $\begin{array}{l}1 \\
2 \\
3 \\
4 \\
5\end{array}$ & $\mathrm{KBC}$ & 1815 & 1817 & LBPT & LY8 & $\{Y \mid 3$ & LYP4 & LYPB & LS & $L T$ \\
\hline \multicolumn{12}{|c|}{18} \\
\hline & $\begin{array}{c}\text { ORDER } \\
1 \\
2 \\
3 \\
4 \\
5\end{array}$ & KAI & KA2 & $\begin{array}{c}k 81 \\
1.7 .27\end{array}$ & $\mathrm{KB2}$ & KB3 & KB4 & K85 & LAI & LA2 & LBI \\
\hline & $\begin{array}{l}1 \\
2 \\
3 \\
4 \\
5\end{array}$ & L82 & $\angle 83$ & LB4 & LB5 & 186 & LB7 & LB9 & LBIO & $|Y|$ & LY2 \\
\hline & $\begin{array}{l}1 \\
2 \\
3 \\
4 \\
5\end{array}$ & LY.3 & $\mathrm{LY}_{4}$ & LYS & LYO & LN & LL & MAI & MA2 & $M B$ & MY \\
\hline & $\begin{array}{l}1 \\
2 \\
3 \\
4 \\
5\end{array}$ & KBI & LBIS & LB17 & $\angle B P 7$ & LY8 & LYI3 & LYPL & LYPB & LS & LT \\
\hline
\end{tabular}




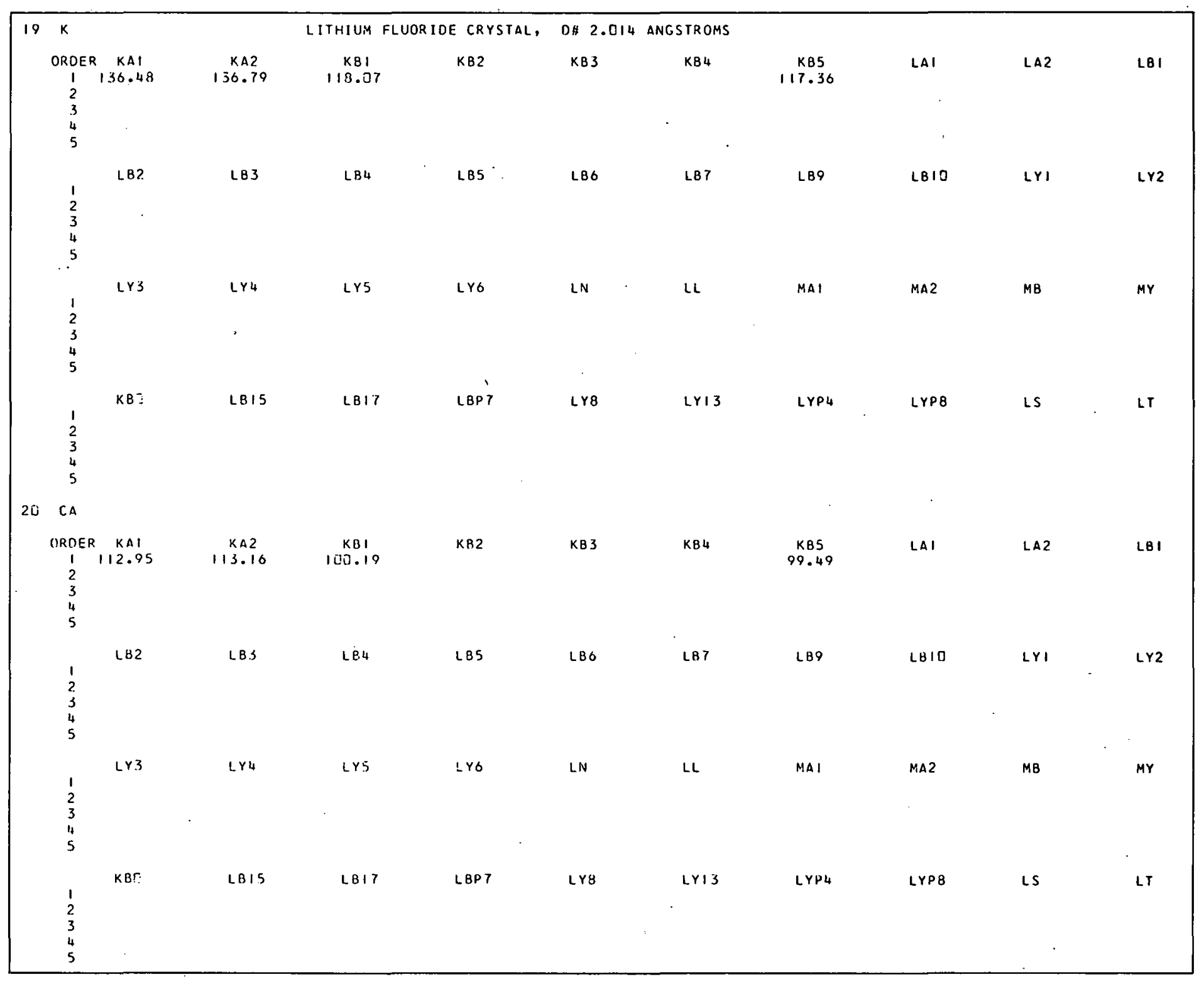




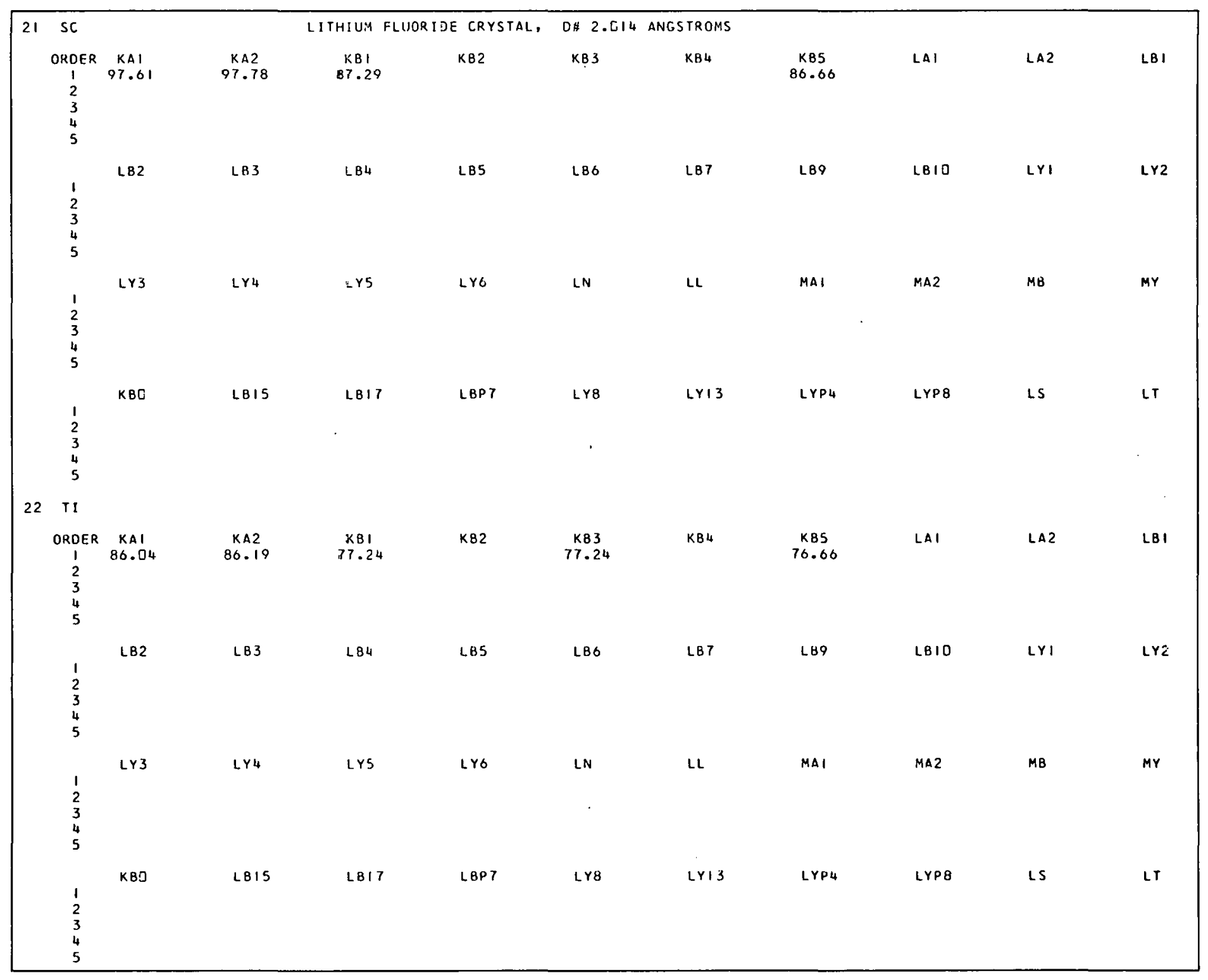




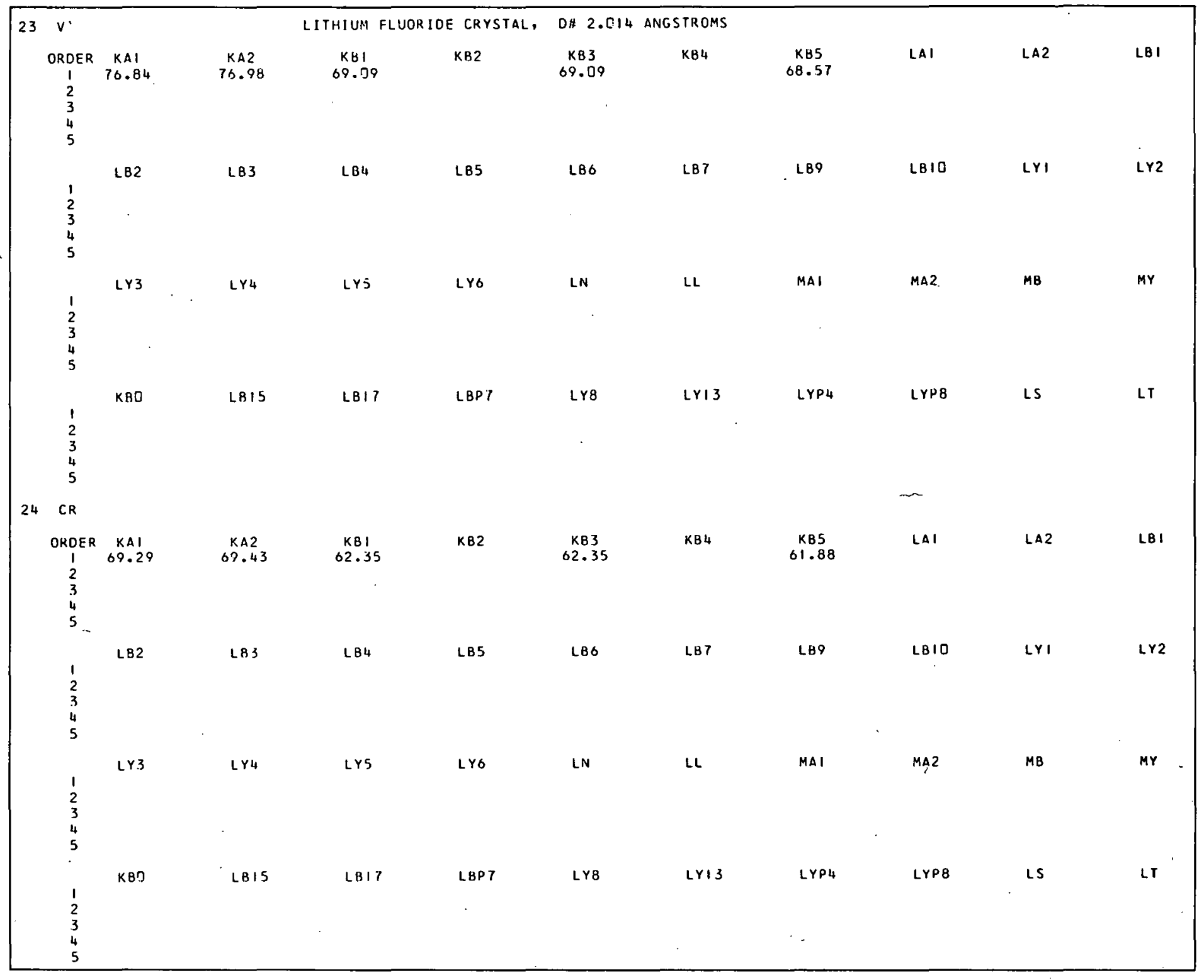




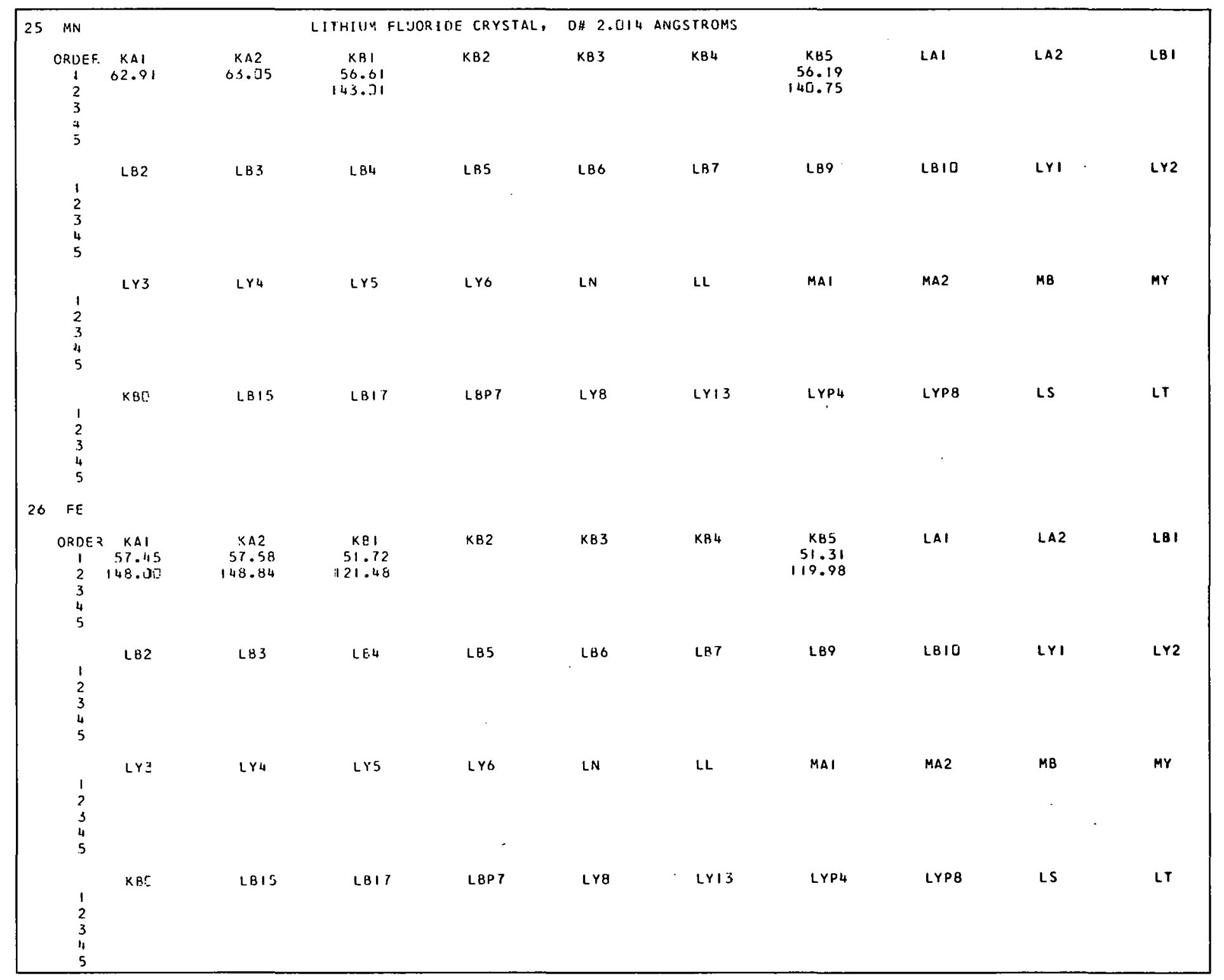




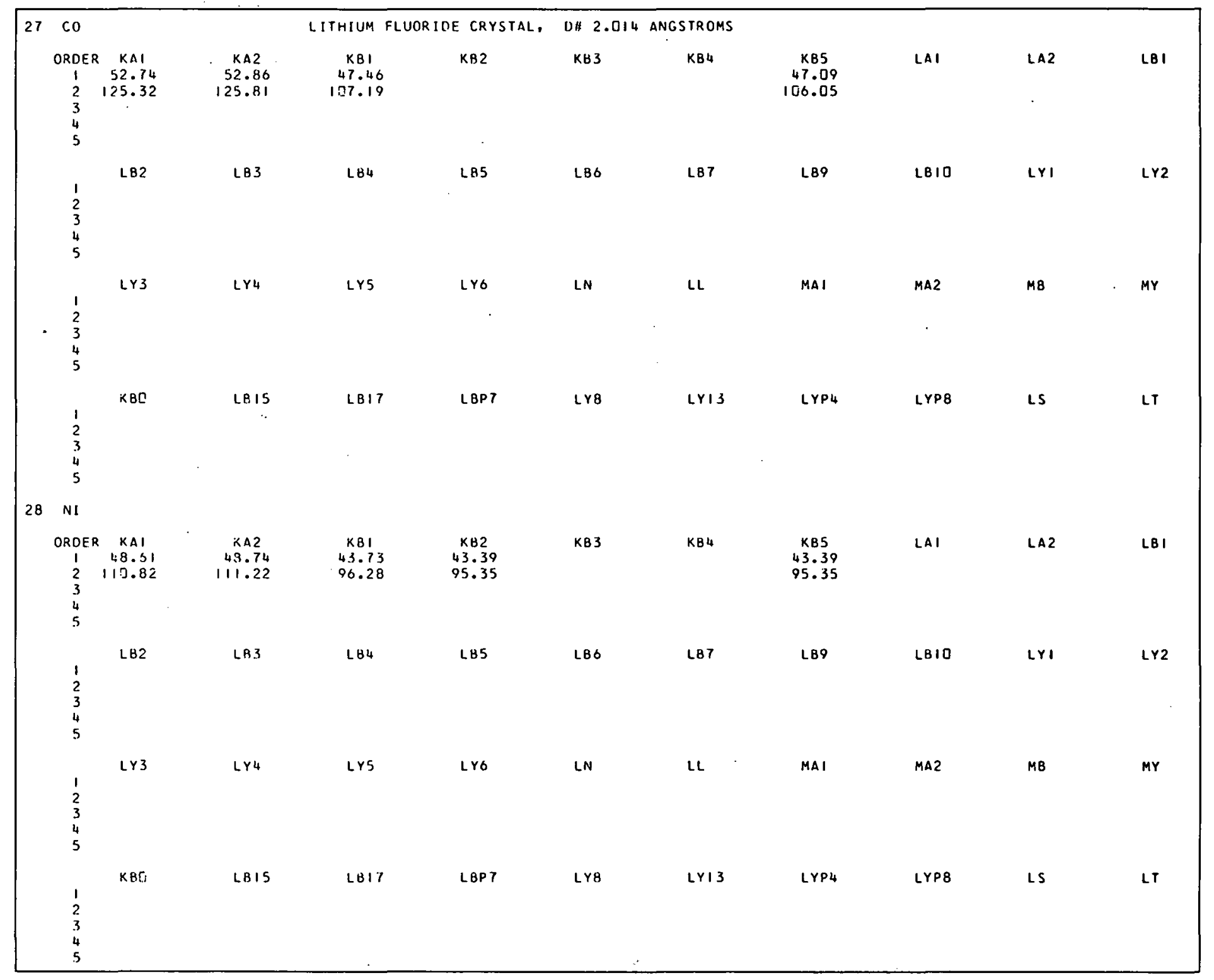




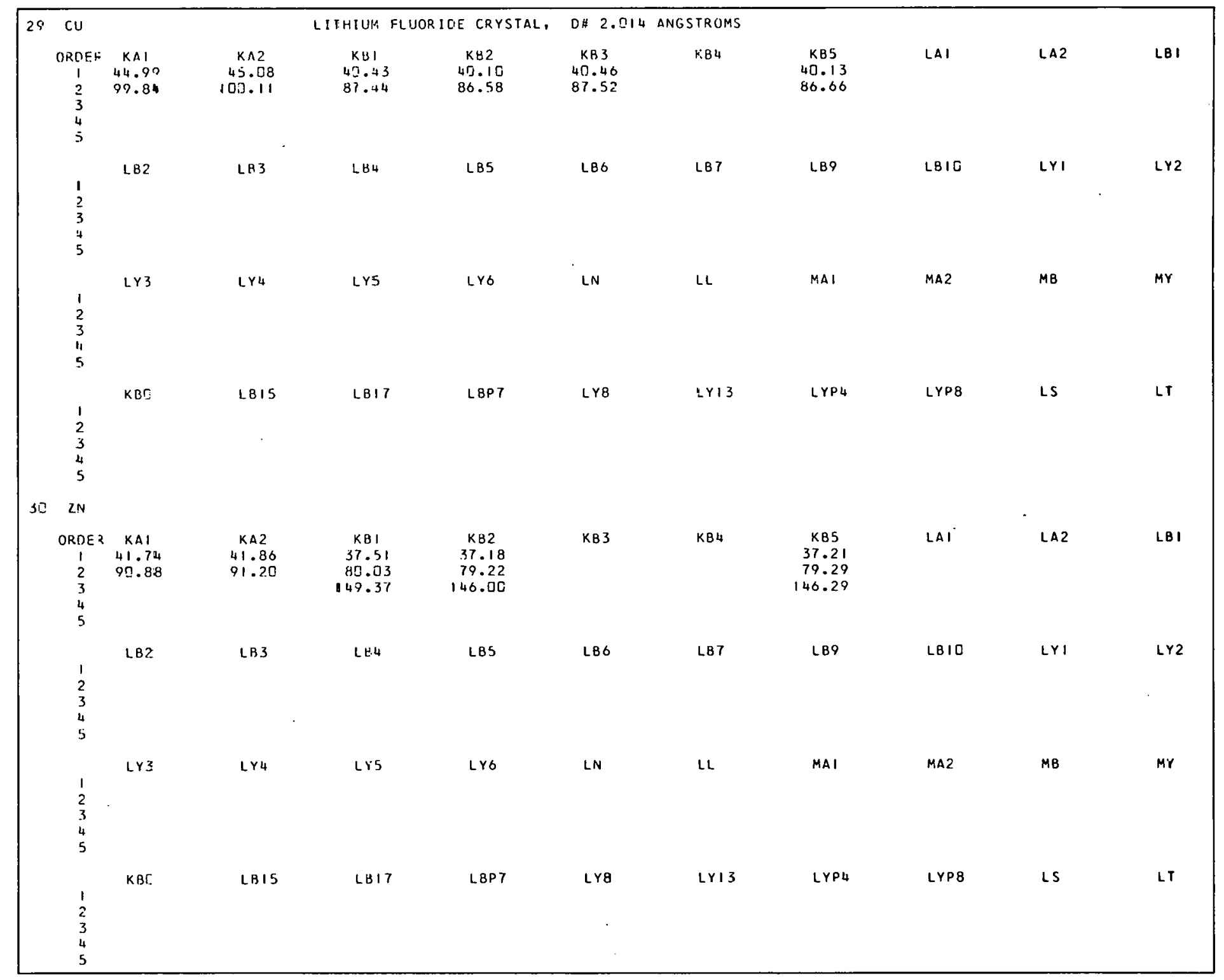




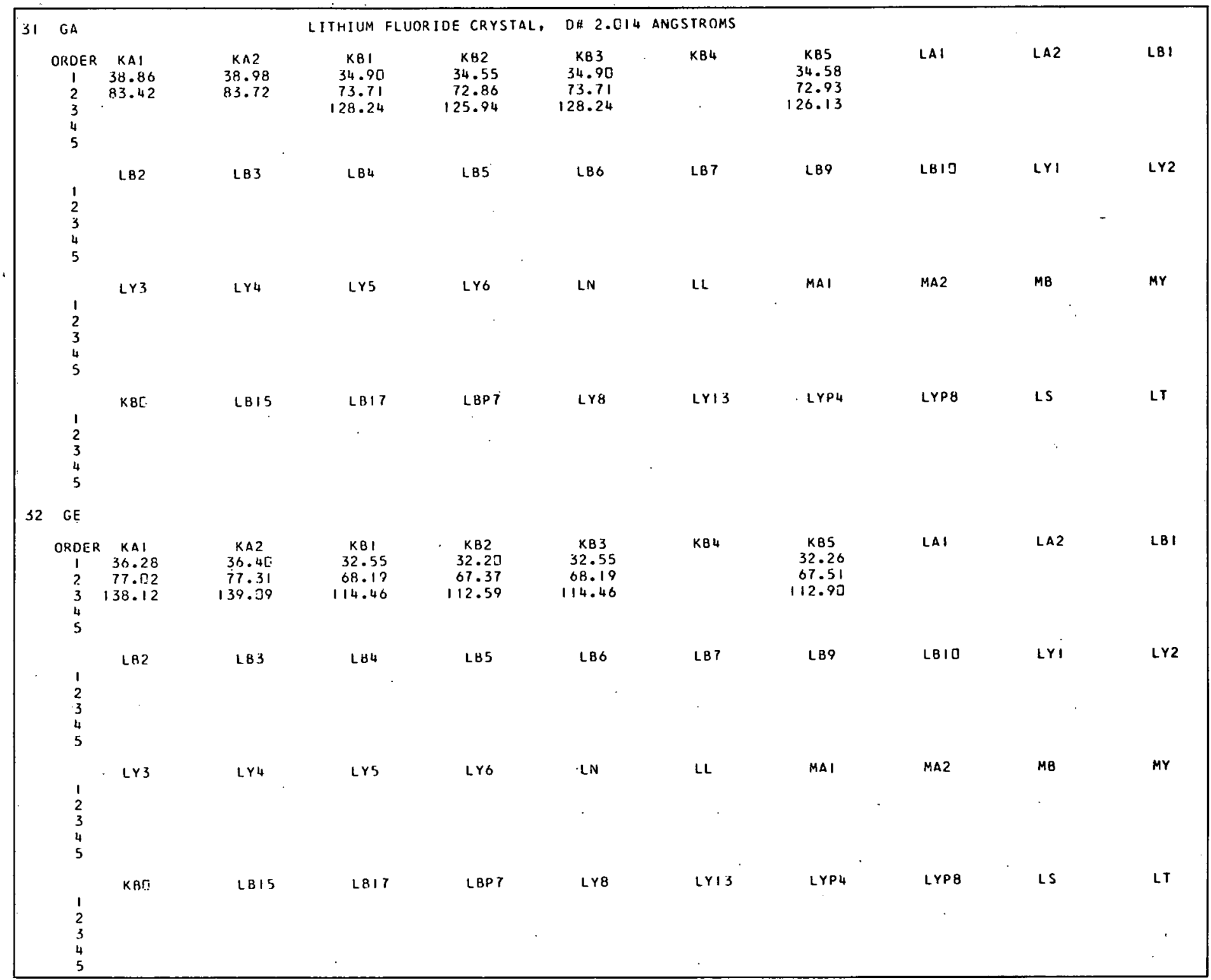




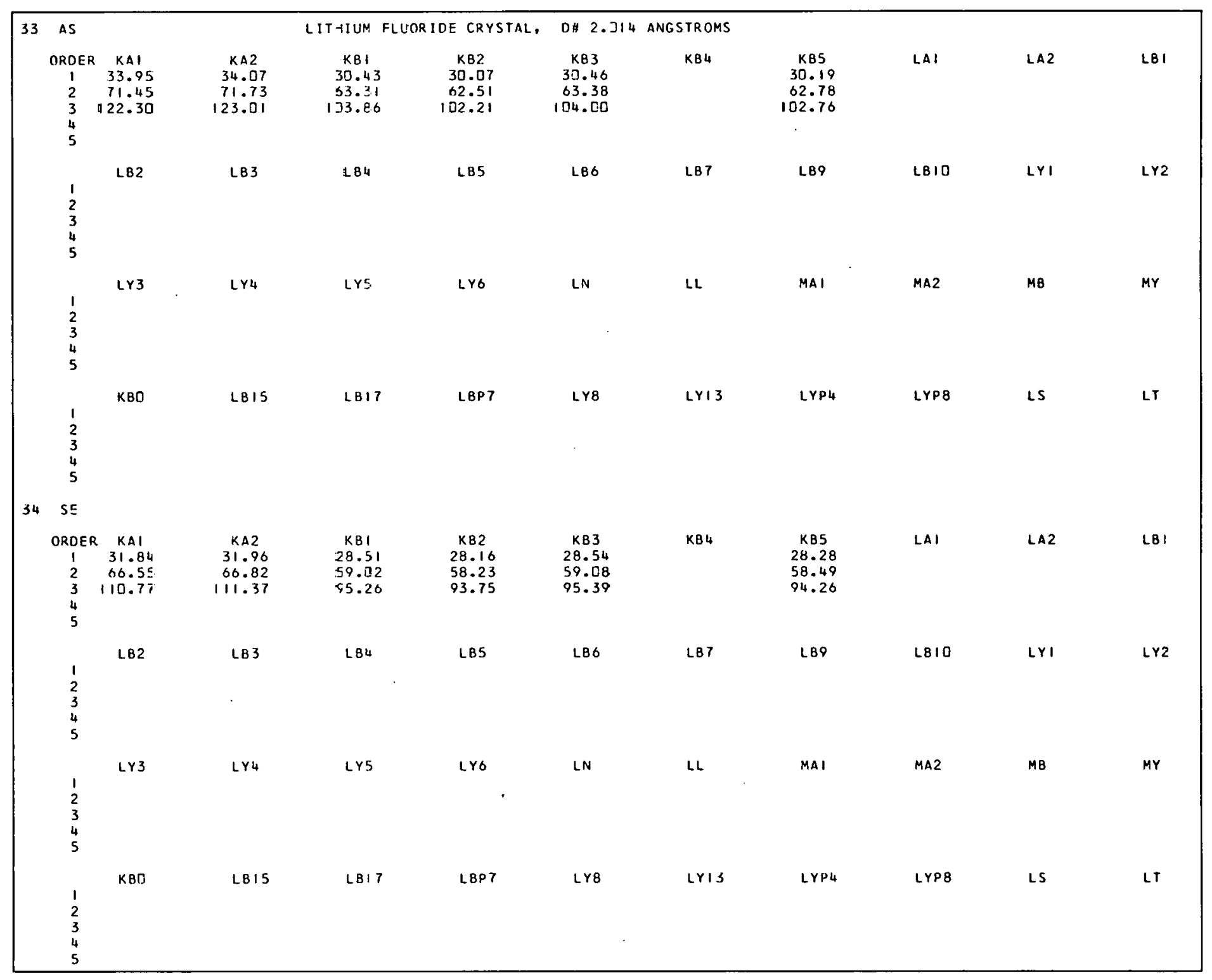




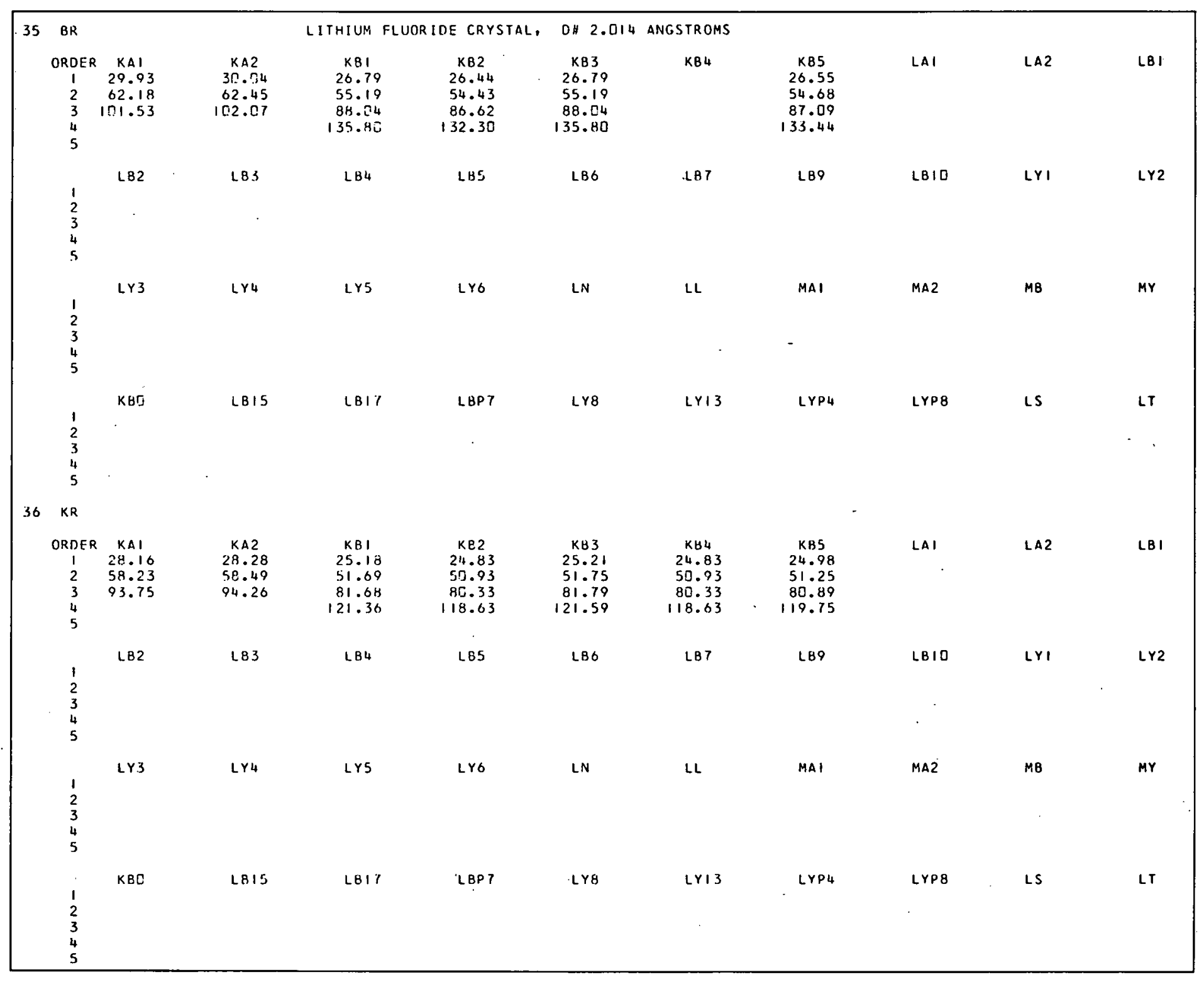




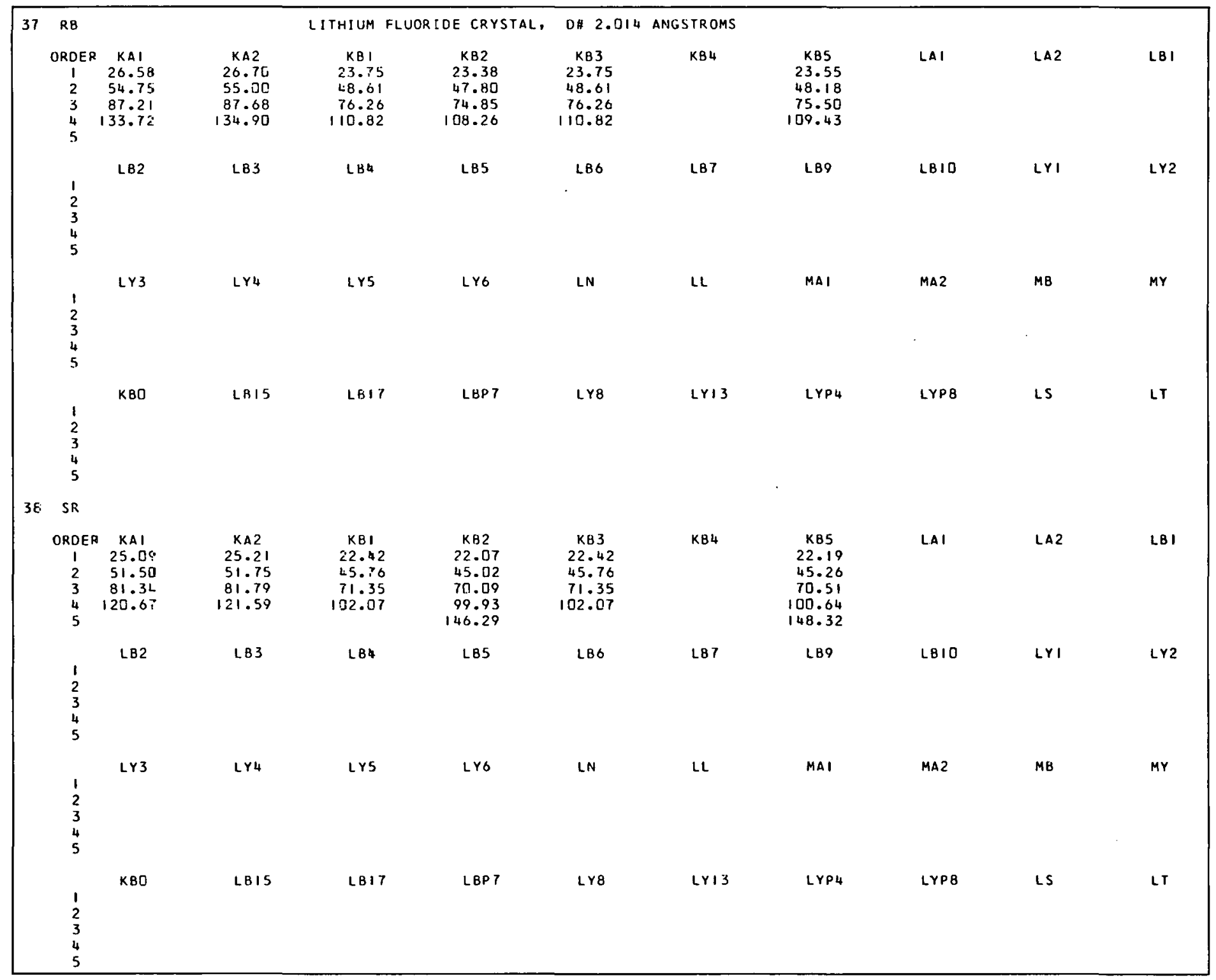




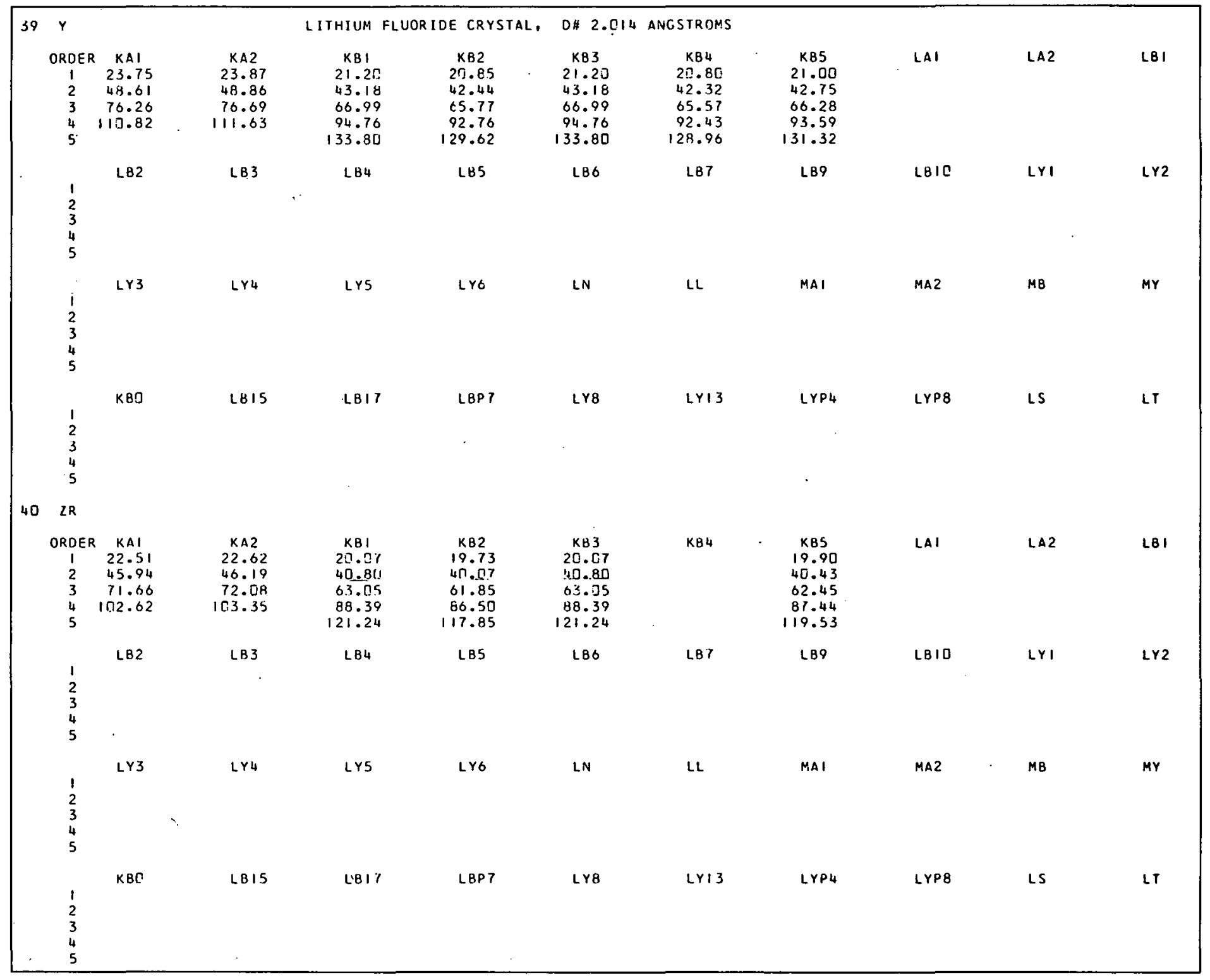




\begin{tabular}{|c|c|c|c|c|c|c|c|c|c|c|c|}
\hline \multirow[t]{5}{*}{41} & NB & & & LITHIUM F & FLUOF. IDE CRYSTAL, & $0 \# 2.014$ & ANGSIROAS & & & & \\
\hline & $\begin{array}{l}\text { OF:DER } \\
1 \\
2 \\
3 \\
4 \\
5\end{array}$ & $\begin{array}{c}\text { KAI } \\
21.35 \\
43.48 \\
67.51 \\
95.60 \\
135.64\end{array}$ & $\begin{array}{r}\text { KA2 } \\
21.46 \\
43.73 \\
67.72 \\
96.28 \\
137.18\end{array}$ & $\begin{array}{c}k 81 \\
19.23 \\
38.62 \\
59.48 \\
82.81 \\
111.53\end{array}$ & $\begin{array}{r}K 82 \\
18.09 \\
37.90 \\
58.30 \\
81.00 \\
108.55\end{array}$ & $\begin{array}{c}k 83 \\
19.03 \\
38.62 \\
59.48 \\
82.81 \\
111.53\end{array}$ & K84 & $\begin{array}{l}K 85 \\
18.86 \\
38.26 \\
58.89 \\
81.90 \\
110.02\end{array}$ & LAI & LA2 & LB। \\
\hline & $\begin{array}{l}1 \\
2 \\
3 \\
4 \\
5\end{array}$ & LB2 & LH3 & LB4 & LBS & L86 & LB7 & L89 & L810 & LYI & LYY \\
\hline & $\begin{array}{l}1 \\
2 \\
3 \\
4 \\
5\end{array}$ & LY. & $L_{Y} 4$ & LYS & LYO & LN & LL & MAI & MA2 & MB & MY \\
\hline & $\begin{array}{l}1 \\
2 \\
3 \\
4 \\
5\end{array}$ & $\mathrm{KBO}$ & LBIS & 4817 & $\angle B P 7$ & LYB & $L Y \mid j$ & LYP4 & LYPB & LS & LT \\
\hline \multirow[t]{5}{*}{42} & mo & & & & & & & & & & \\
\hline & $\begin{array}{c}\text { ORDER } \\
1 \\
2 \\
3 \\
4 \\
5\end{array}$ & $\begin{array}{c}\text { KAI } \\
25.28 \\
41.22 \\
63.75 \\
89.51 \\
123.31\end{array}$ & $\begin{array}{l}K A 2 \\
20.39 \\
41.47 \\
64.15 \\
93.15 \\
124.52\end{array}$ & $\begin{array}{c}K B 1 \\
18.55 \\
36.58 \\
56.16 \\
77.75 \\
103.35\end{array}$ & $\begin{array}{c}K B 2 \\
17.74 \\
35.92 \\
55.10 \\
76.15 \\
100.86\end{array}$ & $\begin{array}{c}K B 3 \\
18.08 \\
36.64 \\
56.26 \\
77.89 \\
103.58\end{array}$ & $\begin{array}{c}K 84 \\
17.71 \\
35.86 \\
55.00 \\
76.00 \\
100.64\end{array}$ & $\begin{array}{c}\text { KB5 } \\
17.91 \\
36.28 \\
55.68 \\
77.02 \\
102.21\end{array}$ & LAI & LA2 & L3 I \\
\hline & $\begin{array}{l}1 \\
2 \\
3 \\
4 \\
5\end{array}$ & $\angle B 2$ & LB3 & L. 84 & LBS & L86 & LB 7 & LB9 & LBIO & $\lfloor Y \mid$ & $\operatorname{LY} 2$ \\
\hline & $\begin{array}{l}1 \\
2 \\
3 \\
4 \\
5\end{array}$ & LY3 & LY4 & LYS & LYo & LN & LL. & MAI & MA2 & MB & MY \\
\hline & $\begin{array}{l}1 \\
2 \\
3 \\
4 \\
5\end{array}$ & KRD & 2815 & LBIT & $\angle B P 7$ & LYE & $L Y \mid 3$ & LYP4 & LYP 8 & LS & LT \\
\hline
\end{tabular}




\begin{tabular}{|c|c|c|c|c|c|c|c|c|c|c|c|}
\hline \multirow[t]{5}{*}{43} & $3 r c$ & & & LITHIUM & FLUORIDE CRYSTAL. & $0 \# 2.014$ & ANGSTROMS & & & & \\
\hline & $\begin{array}{c}\text { ORDER } \\
1 \\
2 \\
3 \\
4 \\
5\end{array}$ & $\begin{array}{r}K A 1 \\
19.24 \\
37.94 \\
60.16 \\
93.88 \\
113.32\end{array}$ & $\begin{array}{c}\text { KA2 } \\
19.32 \\
39.22 \\
60.46 \\
84.34 \\
114.10\end{array}$ & $\begin{array}{l}\text { KB1 } \\
17.19 \\
34.78 \\
53.28 \\
73.43 \\
96.71\end{array}$ & $\begin{array}{l}K 82 \\
16.85 \\
34.07 \\
52.13 \\
71.7 .3 \\
94.17\end{array}$ & $\begin{array}{c}K 83 \\
17.16 \\
34.72 \\
53.18 \\
73.29 \\
96.49\end{array}$ & $\begin{array}{l}K B 4 \\
16.85 \\
34.07 \\
52.1 .3 \\
71.73 \\
94.17\end{array}$ & K85 & (A) & LAZ & LBI \\
\hline & $\begin{array}{l}1 \\
2 \\
3 \\
4 \\
5\end{array}$ & LB2 & LB3 & L84 & LB5 & 186 & LB 7 & L89 & L810 & $\lfloor Y \mid$ & LYZ \\
\hline & $\begin{array}{l}1 \\
2 \\
3 \\
4 \\
5\end{array}$ & LY3 & LY4 & LYS & LYG & LN & LL & MAI & MA2 & MB & MY \\
\hline & $\begin{array}{l}1 \\
2 \\
3 \\
4 \\
5\end{array}$ & KUS & LBIS & LUIT & LBP 7 & LY8 & LYI3 & $\begin{array}{l}\text { LYPL } \\
\cdot \\
\cdots\end{array}$ & LYP8 & LS & LT \\
\hline \multicolumn{12}{|c|}{ RU } \\
\hline & $\begin{array}{c}\text { ORDER } \\
1 \\
. \quad 2 \\
3 \\
4 \\
5\end{array}$ & $\begin{array}{r}K A 1 \\
18.37 \\
37.24 \\
57.23 \\
79.36 \\
195.91\end{array}$ & $\begin{array}{c}K A 2 \\
18.49 \\
37.48 \\
57.62 \\
79.96 \\
106.86\end{array}$ & $\begin{array}{l}\text { KB1 } \\
16.33 \\
33.35 \\
50.43 \\
69.23 \\
90.47\end{array}$ & $\begin{array}{l}K 82 \\
16.04 \\
32.41 \\
49.49 \\
67.85 \\
88.47\end{array}$ & $\begin{array}{l}K 83 \\
16.36 \\
33.06 \\
50.52 \\
69.36 \\
90.68\end{array}$ & KBH & $\begin{array}{l}k 85 \\
16.21 \\
32.76 \\
50.05 \\
68.07 \\
89.67\end{array}$ & LAI & LA2 & LBI \\
\hline & $\begin{array}{l}1 \\
2 \\
3 \\
4 \\
5\end{array}$ & LB2 & LA? & LB4 & LES & L86 & 487 & L89 & LBIO & LYI & LY2 \\
\hline & $\begin{array}{l}1 \\
2 \\
3 \\
4 \\
5\end{array}$ & LY3 & LY4 & LYS & LYo & LN & $L L$ & MAI & MA2 & MB & MY \\
\hline & $\begin{array}{l}1 \\
2 \\
3 \\
4 \\
5\end{array}$ & KEIJ & 2815 & LBIT & LBP7 & LYB & LYI3 & LYP4 & LYPB & LS & LT \\
\hline
\end{tabular}




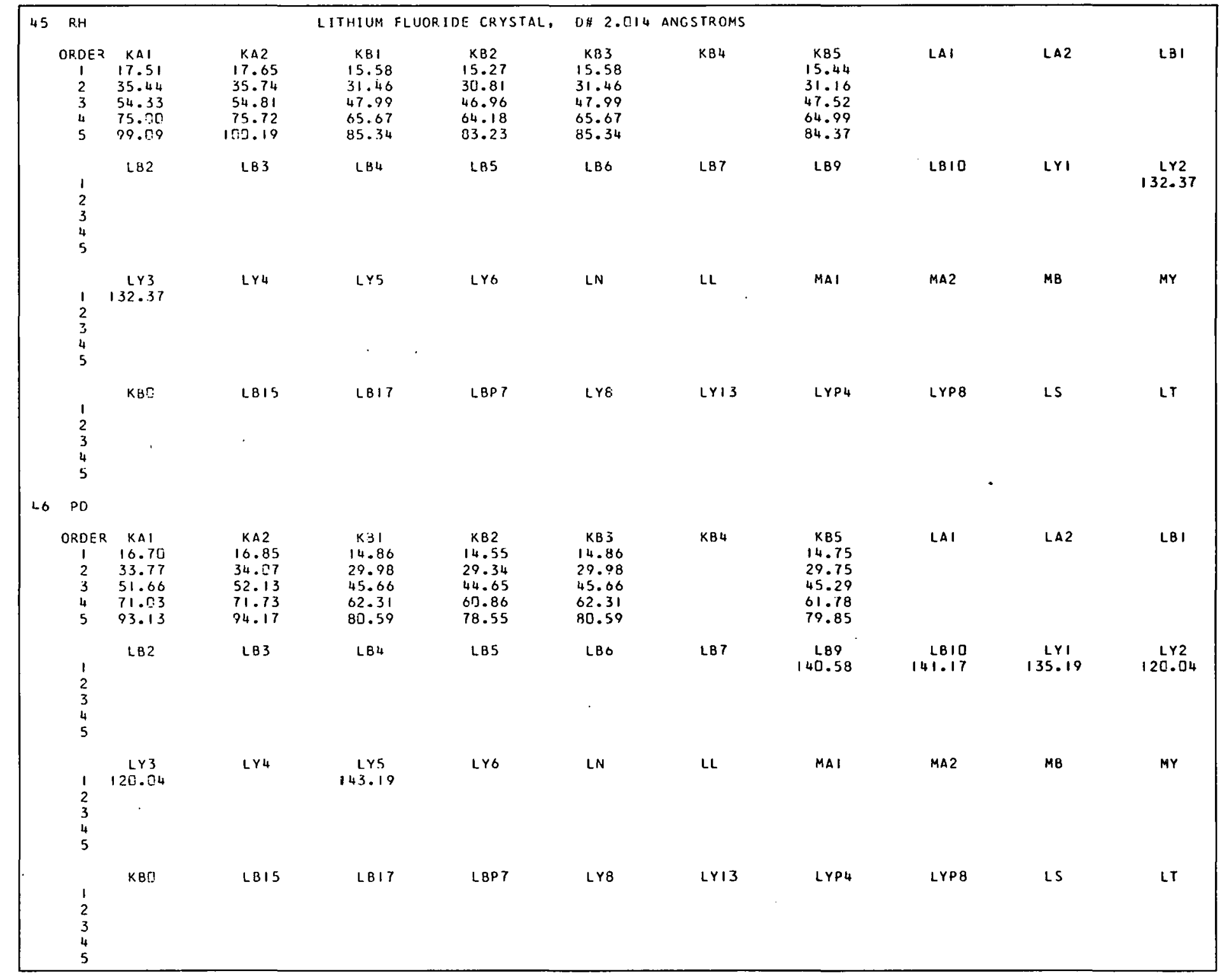




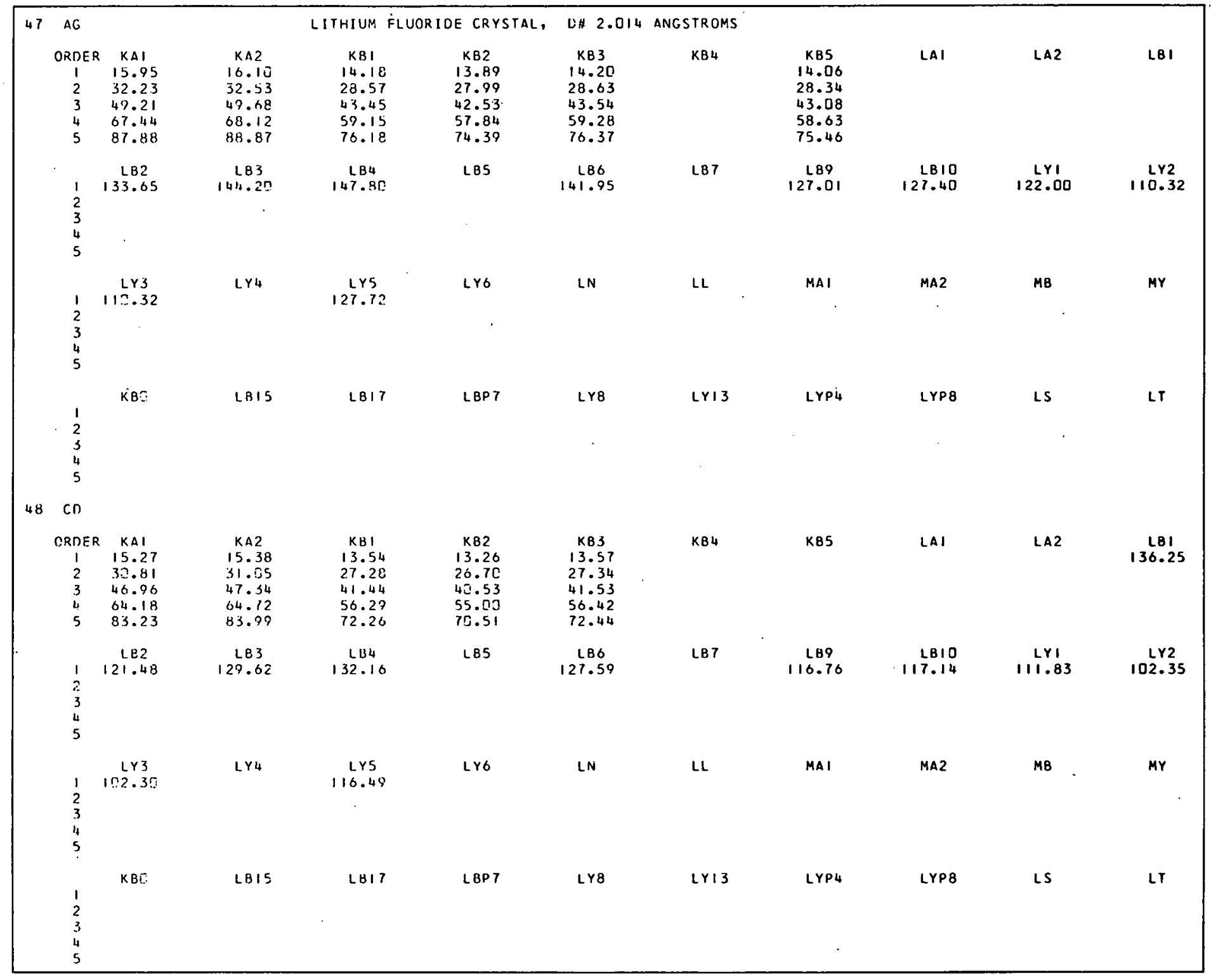




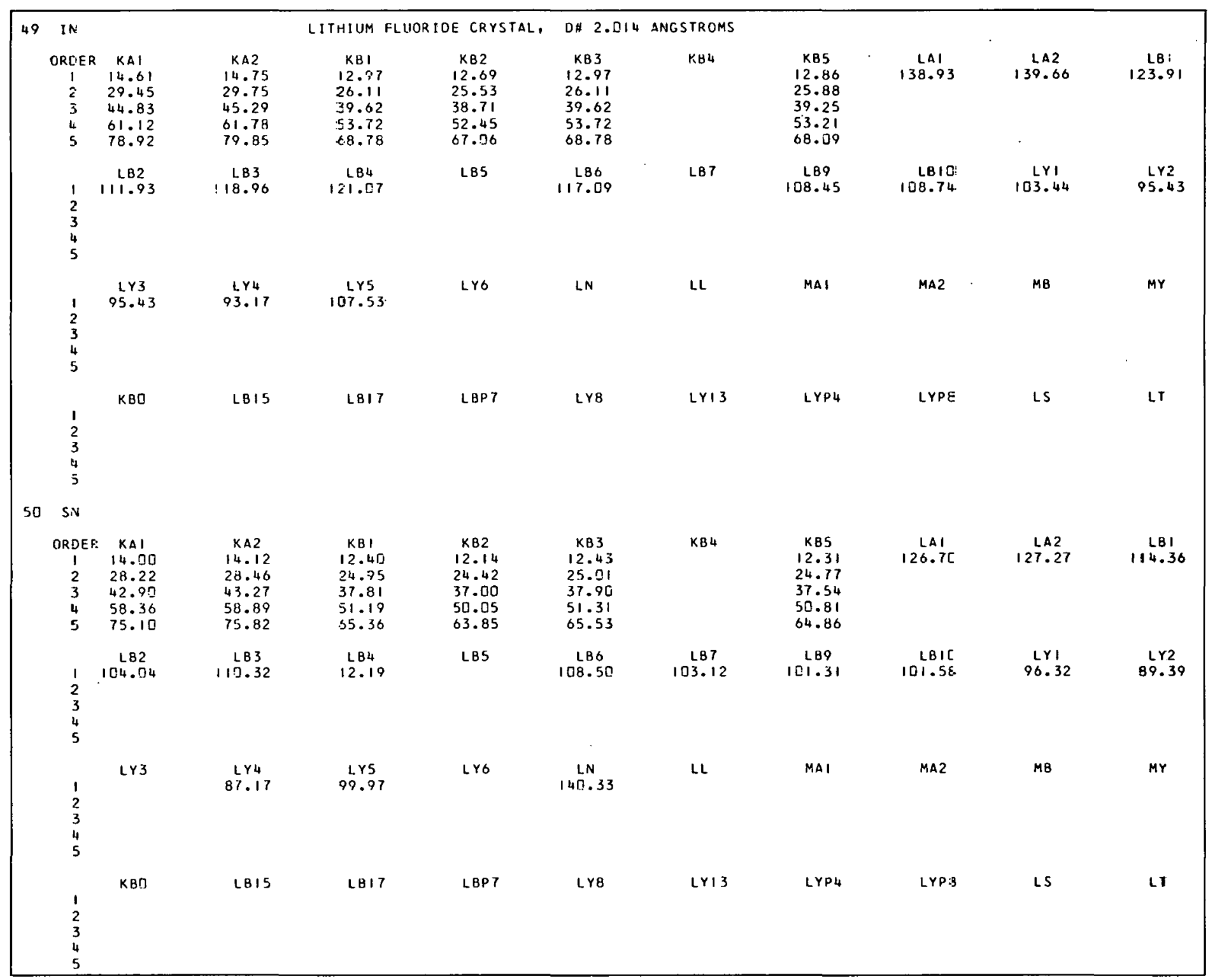




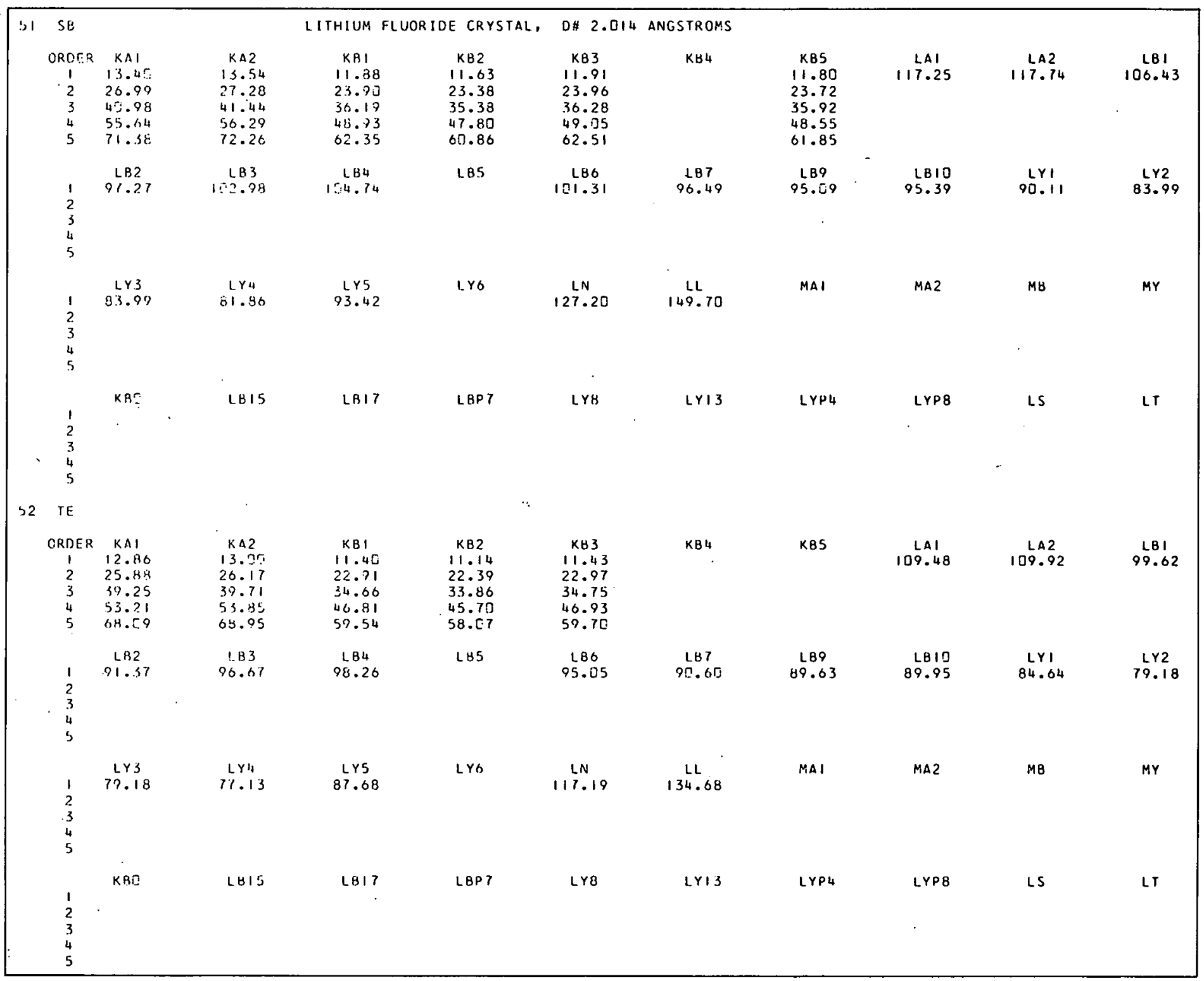




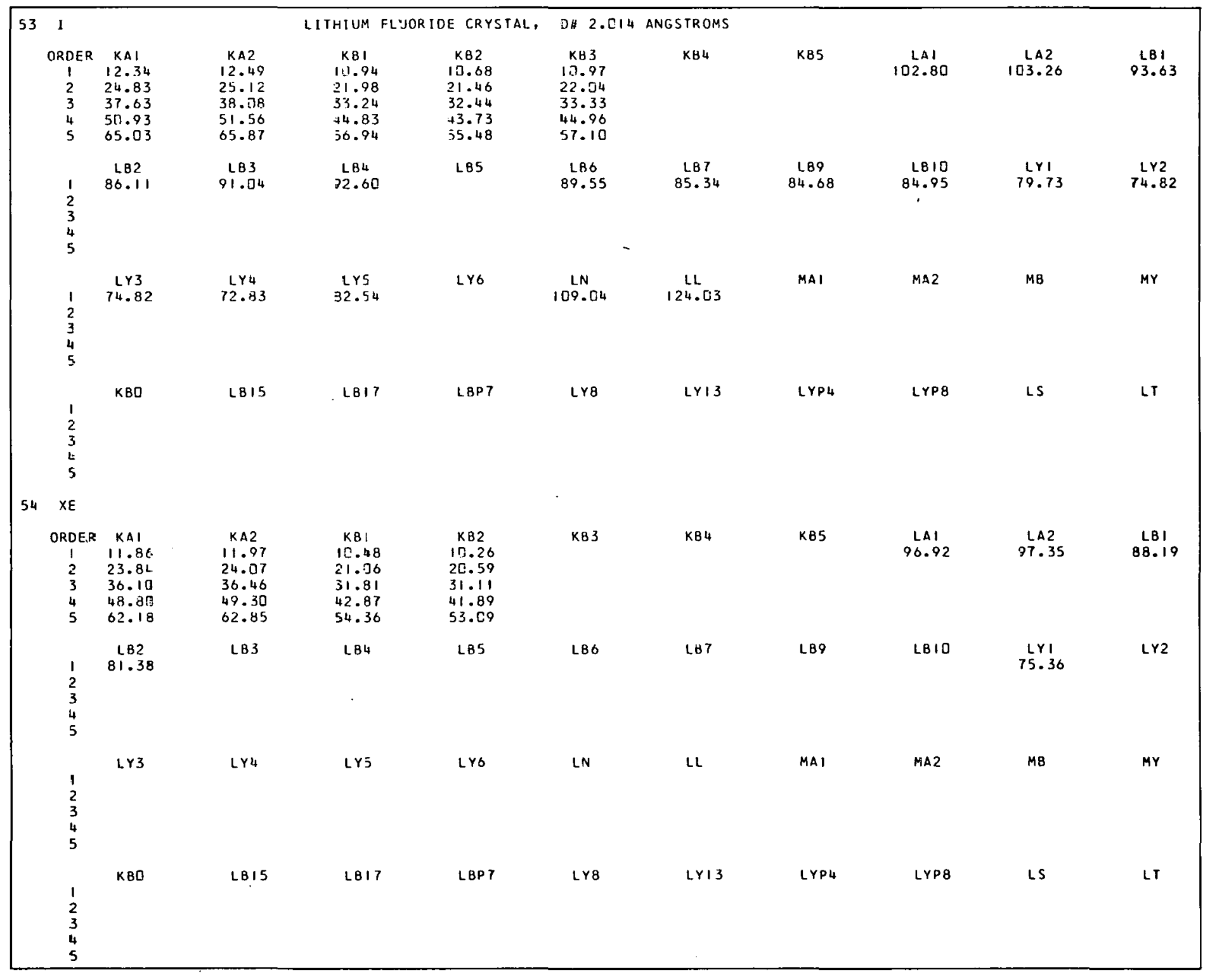




\begin{tabular}{|c|c|c|c|c|c|c|c|c|c|c|c|}
\hline \multirow[t]{5}{*}{55} & CS & & & LITHIUM F & DE CRYSTAL, & DH 2.014 & ANGSTROMS & & & & \\
\hline & $\begin{array}{c}\text { ORDER } \\
1 \\
2 \\
3 \\
4 \\
5\end{array}$ & $\begin{array}{c}K A 1 \\
11.4 C \\
22.91 \\
34.66 \\
46.81 \\
59.54\end{array}$ & $\begin{array}{l}K A 2 \\
11.54 \\
23.25 \\
35.11 \\
47.43 \\
67.36\end{array}$ & $\begin{array}{l}K B 1 \\
1.2 .08 \\
217.25 \\
30.57 \\
41.16 \\
52.13\end{array}$ & $\begin{array}{l}K B 2 \\
9.86 \\
17.78 \\
29.87 \\
49.19 \\
50.87\end{array}$ & $\begin{array}{c}K B 3 \\
19.11 \\
20.30 \\
30.66 \\
41.28 \\
52.29\end{array}$ & KB4 & KBS & $\begin{array}{c}\text { LAL } \\
91.77\end{array}$ & $\begin{array}{l}\text { LA2. } \\
92.18\end{array}$ & $\begin{array}{c}\text { LBI } \\
83.53\end{array}$ \\
\hline & $\begin{array}{l}1 \\
2 \\
3 \\
4 \\
5\end{array}$ & $\begin{array}{c}\angle B 2 \\
77.13\end{array}$ & $\begin{array}{c}283 \\
81.45\end{array}$ & $\begin{array}{c}L 84 \\
82.98\end{array}$ & LB5 & $\begin{array}{c}\text { LB6 } \\
80.14\end{array}$ & $\begin{array}{c}\text { LB7 } \\
76.18\end{array}$ & $\begin{array}{c}\text { LB9 } \\
75.93\end{array}$ & $\begin{array}{r}L B 10 \\
76.44\end{array}$ & $\begin{array}{c}\lfloor Y 1 \\
71.31\end{array}$ & $\begin{array}{l}\text { LY2 } \\
67.47\end{array}$ \\
\hline & $\begin{array}{l}1 \\
2 \\
3 \\
4 \\
5\end{array}$ & $\begin{array}{c}\text { LY3 } \\
67.33\end{array}$ & $\begin{array}{r}\mathrm{LY4} \\
65.33\end{array}$ & $\begin{array}{c}\text { LYS } \\
73.75\end{array}$ & LYO & $\begin{array}{l}\text { LN } \\
95.98\end{array}$ & $\begin{array}{c}L 2 \\
108.40\end{array}$ & MAI & MA2 & MB & MY \\
\hline & $\begin{array}{l}1 \\
2 \\
3 \\
4 \\
5\end{array}$ & $K B C$. & $L B 15$ & $\angle B \mid 7$ & $\angle B P 7$ & LY8 & $2 Y \mid 3$ & LYP4 & LYPB & LS & LT \\
\hline \multirow[t]{5}{*}{56} & BA & & & & & & & & & & \\
\hline & $\begin{array}{c}\text { ORDER } \\
1 \\
2 \\
3 \\
4 \\
5\end{array}$ & $\begin{array}{c}K A 1 \\
16.97 \\
22.5 .4 \\
33.33 \\
414.96 \\
57.17\end{array}$ & $\begin{array}{l}\text { KA2 } \\
11.11 \\
22.33 \\
33.77 \\
45.57 \\
57.91\end{array}$ & $\begin{array}{l}\text { KBI } \\
9.71 \\
19.55 \\
29.43 \\
39.59 \\
50.08\end{array}$ & $\begin{array}{c}K B 2 \\
9.48 \\
19.03 \\
28.72 \\
38.62 \\
43.83\end{array}$ & $\begin{array}{c}K B 3 \\
9.71 \\
19.50 \\
29.43 \\
39.59 \\
50.08\end{array}$ & $K 84$ & $\begin{array}{c}K B 5 \\
9.63 \\
19.32 \\
29.16 \\
39.22 \\
49.61\end{array}$ & $\begin{array}{l}\text { LAL } \\
87.09\end{array}$ & $\begin{array}{c}\text { LA2 } \\
87.48\end{array}$ & $\begin{array}{l}\text { LBI } \\
19.18\end{array}$ \\
\hline & $\begin{array}{l}1 \\
2 \\
3 \\
4 \\
5\end{array}$ & $\begin{array}{c}\angle 82 \\
73.29\end{array}$ & $\begin{array}{c}183 \\
77.31\end{array}$ & $\begin{array}{c}\text { LB4 } \\
78.74\end{array}$ & LB5 & $\begin{array}{c}\text { LB6 } \\
76.08\end{array}$ & 187 & $\begin{array}{c}\text { LB9 } \\
72.30\end{array}$ & $\begin{array}{r}\angle B 10 \\
72.68\end{array}$ & $\begin{array}{c}\text { LYI } \\
67.61\end{array}$ & $\begin{array}{l}\text { LY2 } \\
64.12\end{array}$ \\
\hline & $\begin{array}{l}1 \\
2 \\
3 \\
4 \\
5\end{array}$ & $\begin{array}{c}\operatorname{LY3} \\
63.98\end{array}$ & $\begin{array}{l}L Y 4 \\
62.01\end{array}$ & $\begin{array}{l}\text { LY5 } \\
69.92\end{array}$ & LYS & $\begin{array}{l}\text { LN } \\
90.56\end{array}$ & $\begin{array}{c}\mathrm{LL} \\
102.21\end{array}$ & MAI & MA2 & $M B$ & MY \\
\hline & $\begin{array}{l}1 \\
1 \\
2 \\
3 \\
4 \\
5\end{array}$ & KBC & $\angle B 15$ & LBIT & LBP 7 & LY8 & $\begin{array}{c}\text { LYI3 } \\
.\end{array}$ & LYP4 & LYPB & LS & $L T$ \\
\hline
\end{tabular}




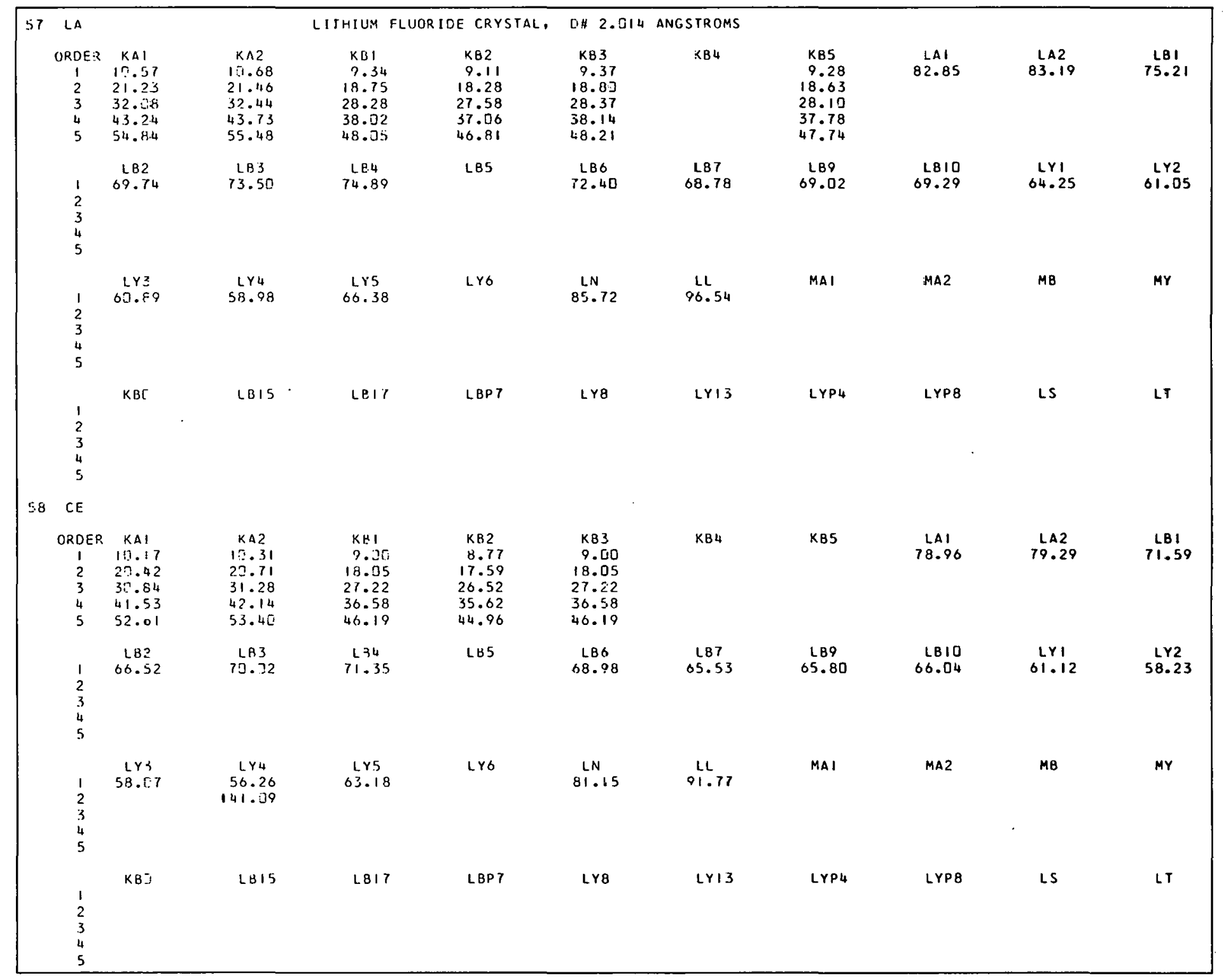




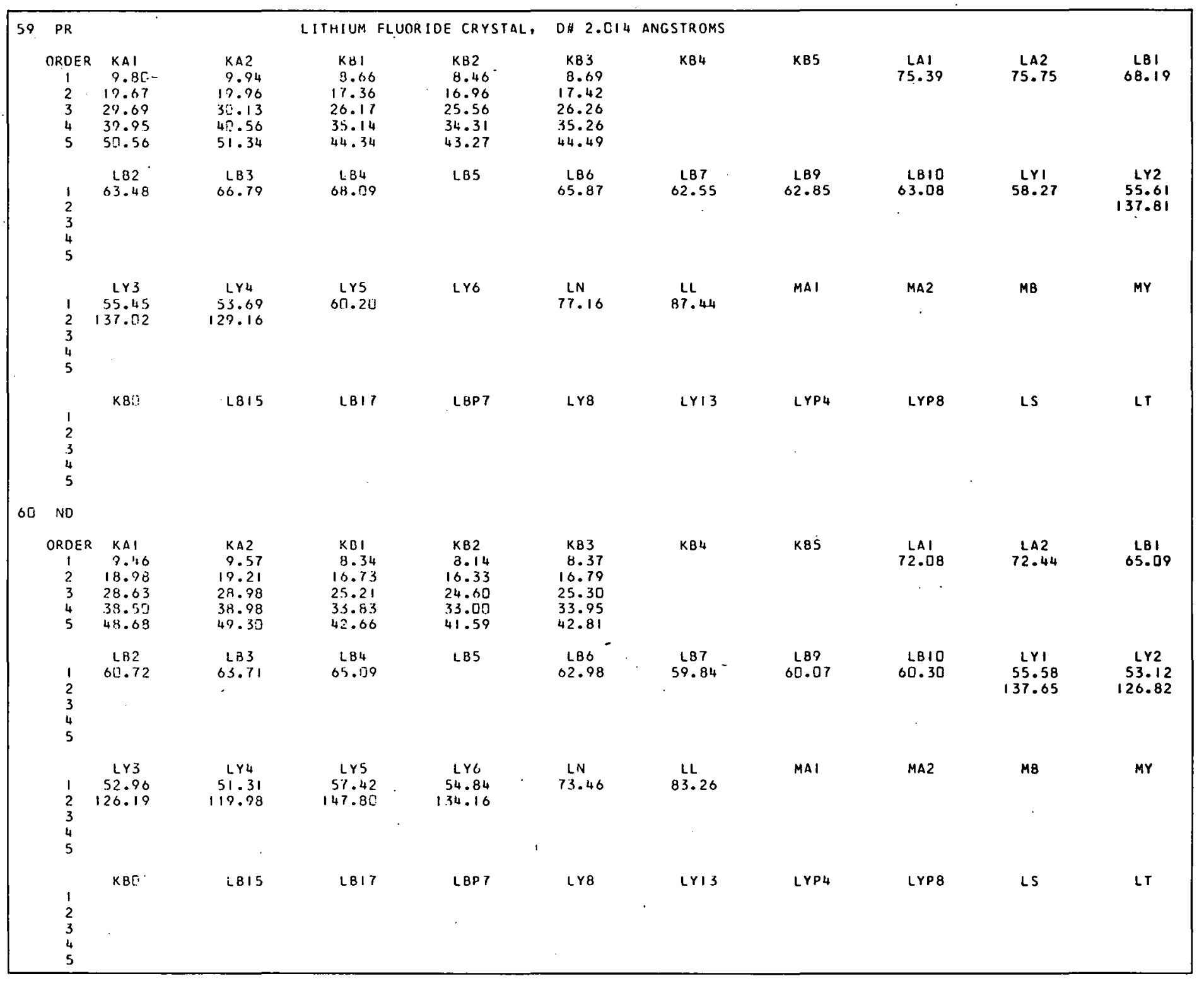




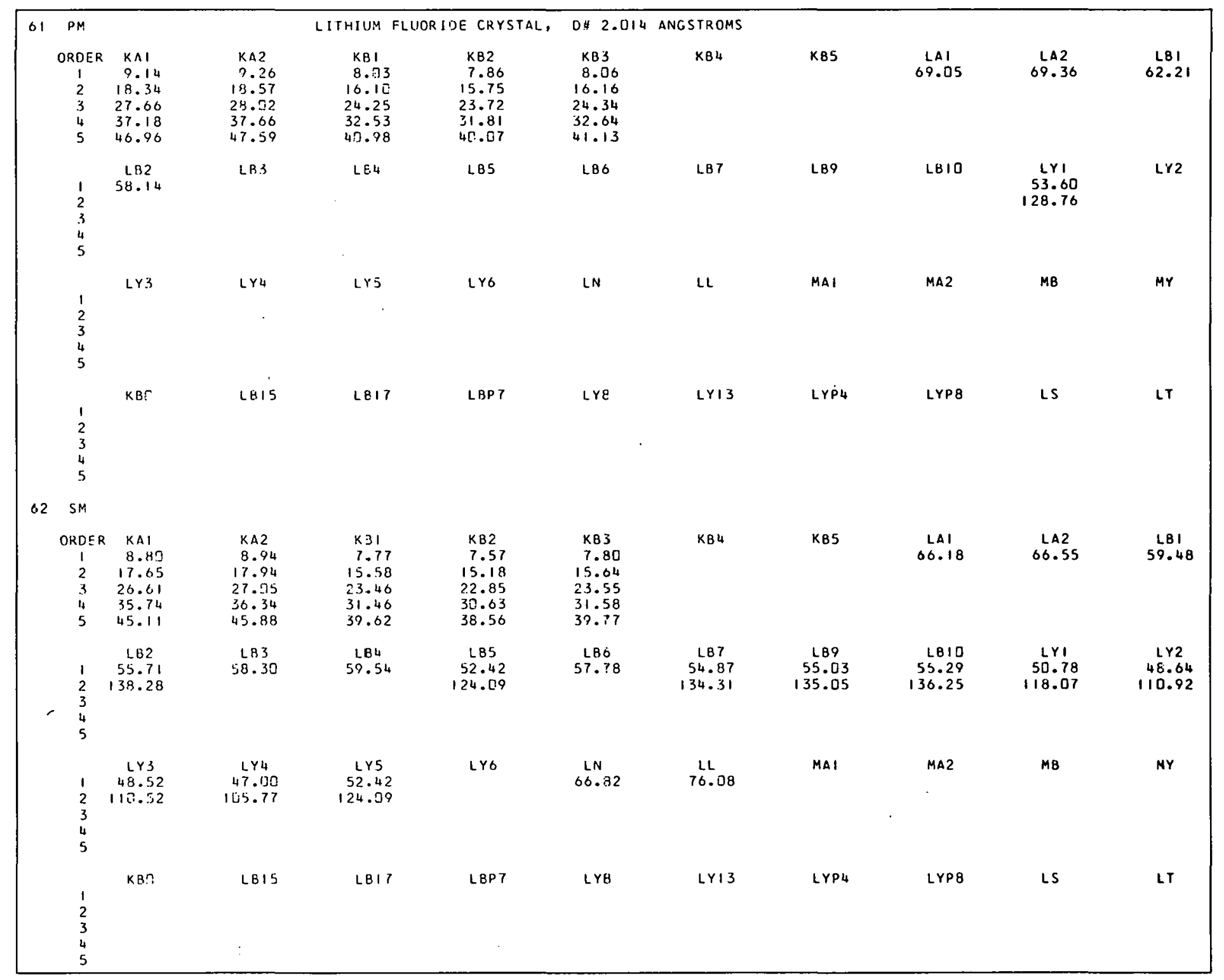




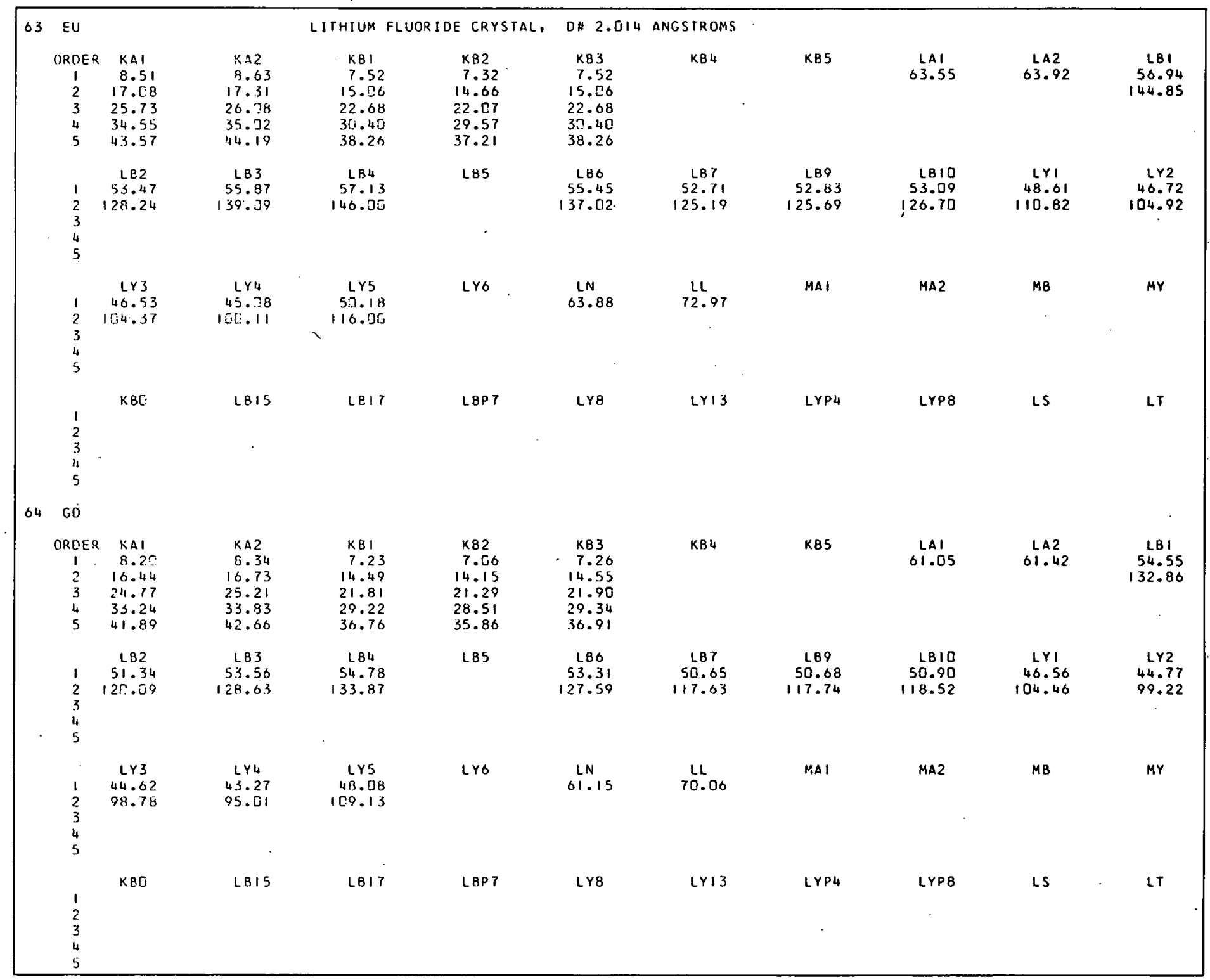




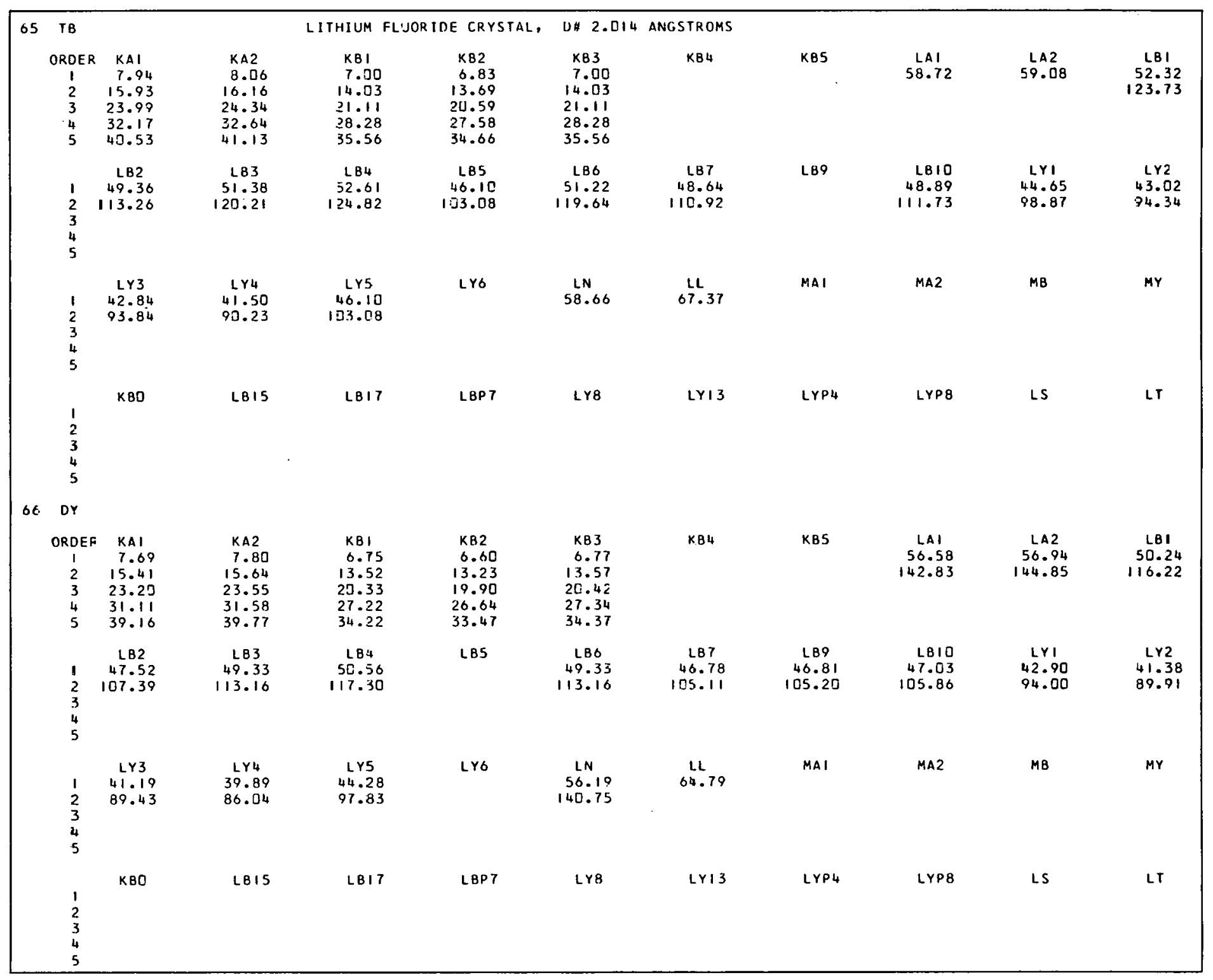




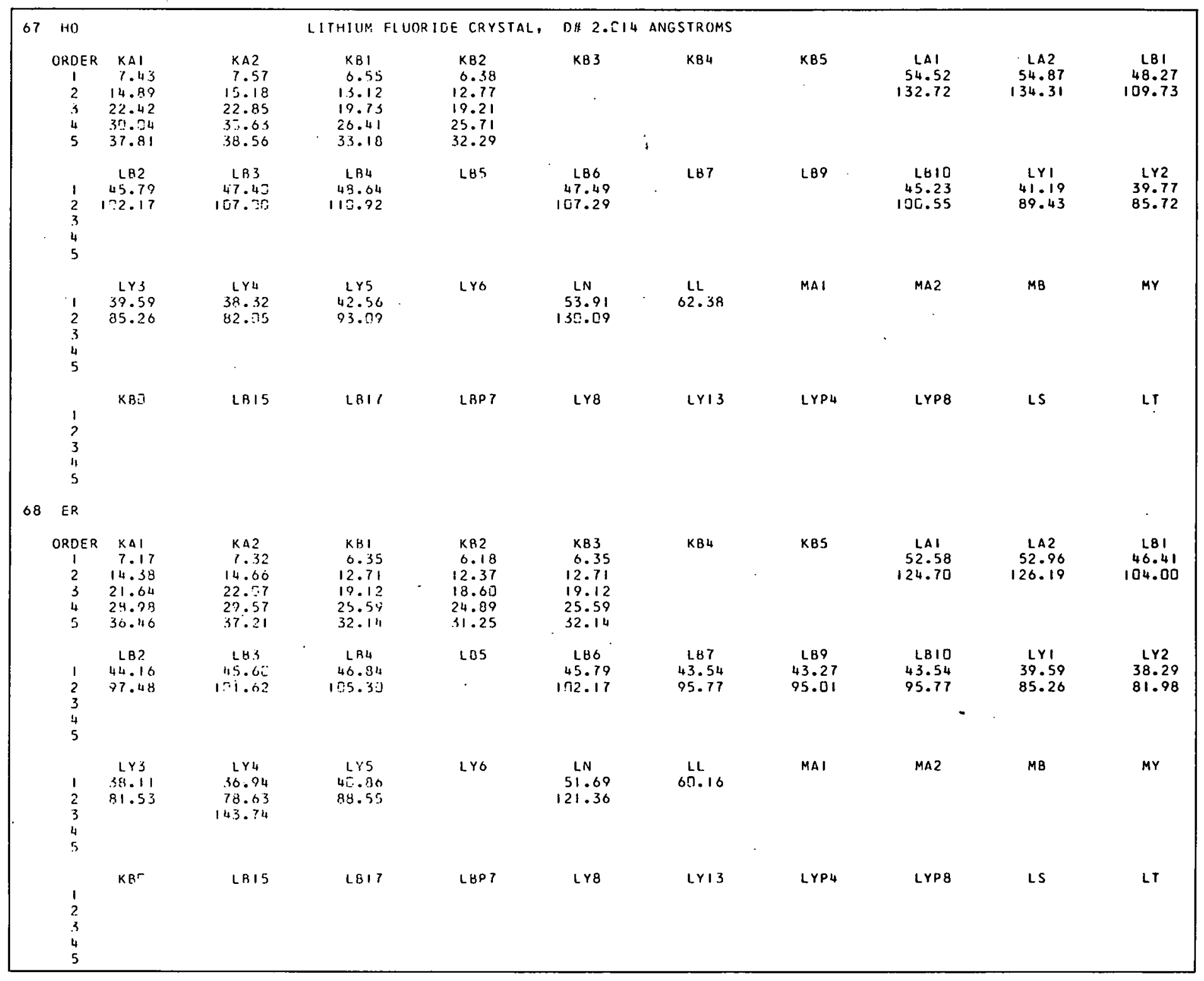




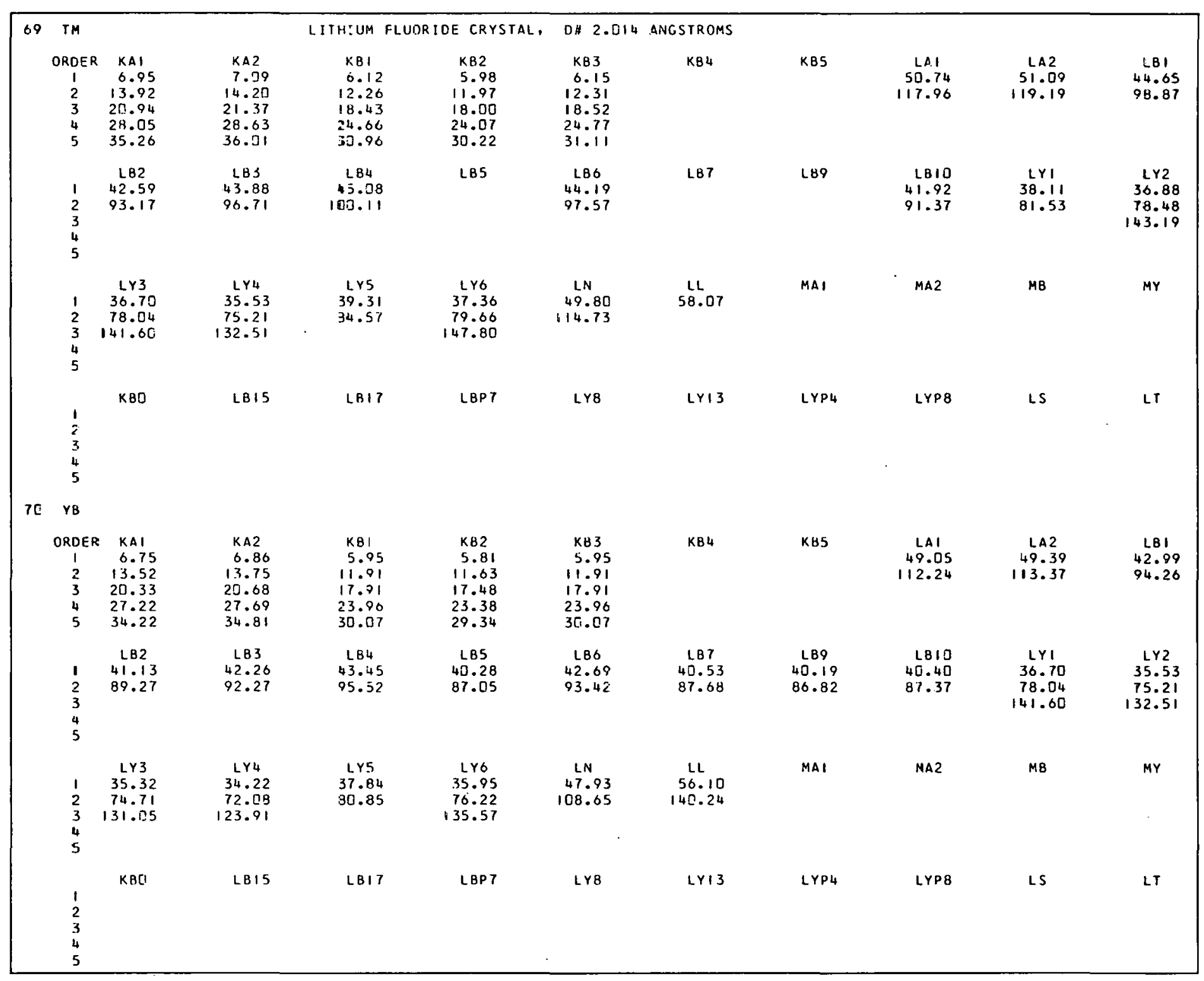




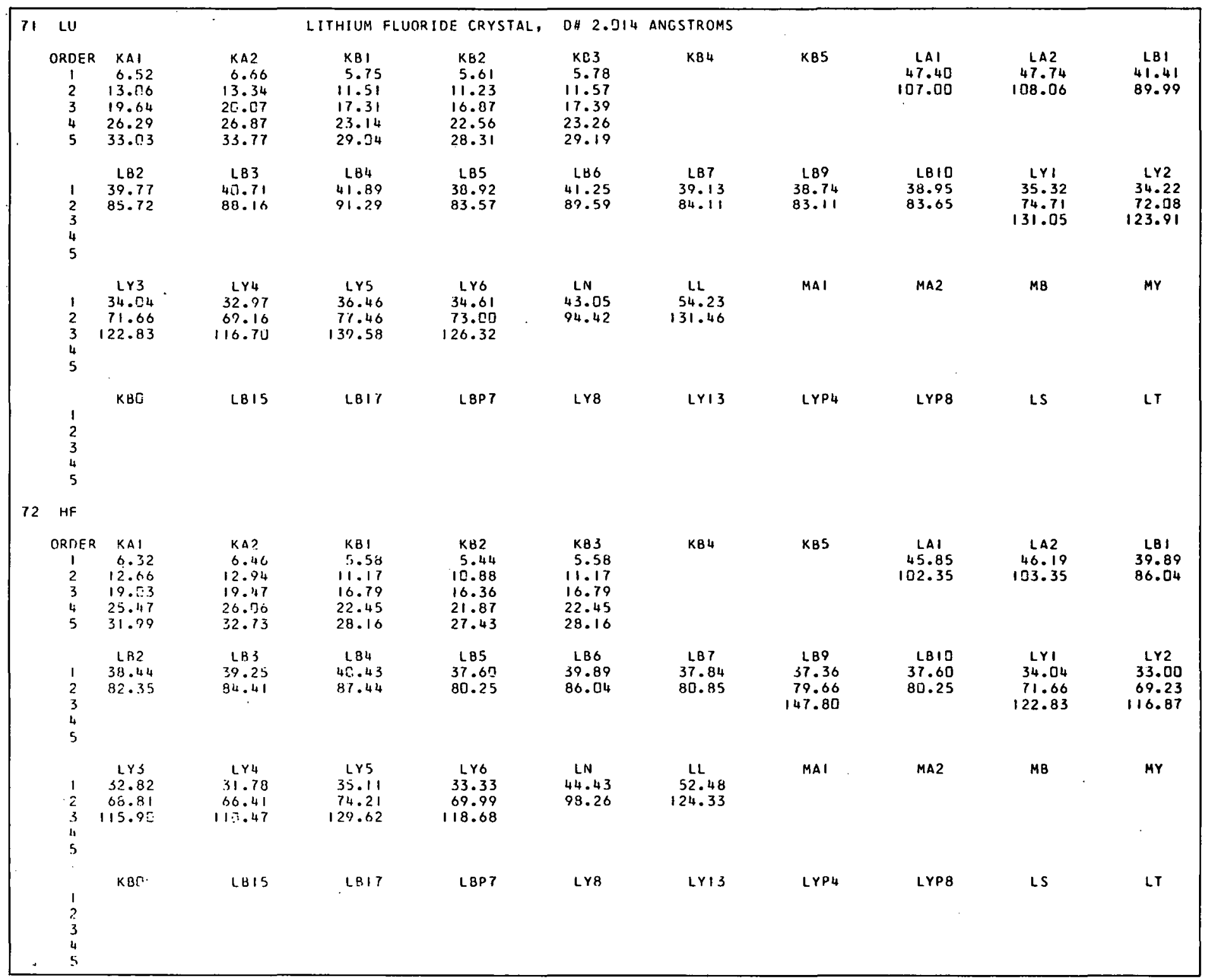




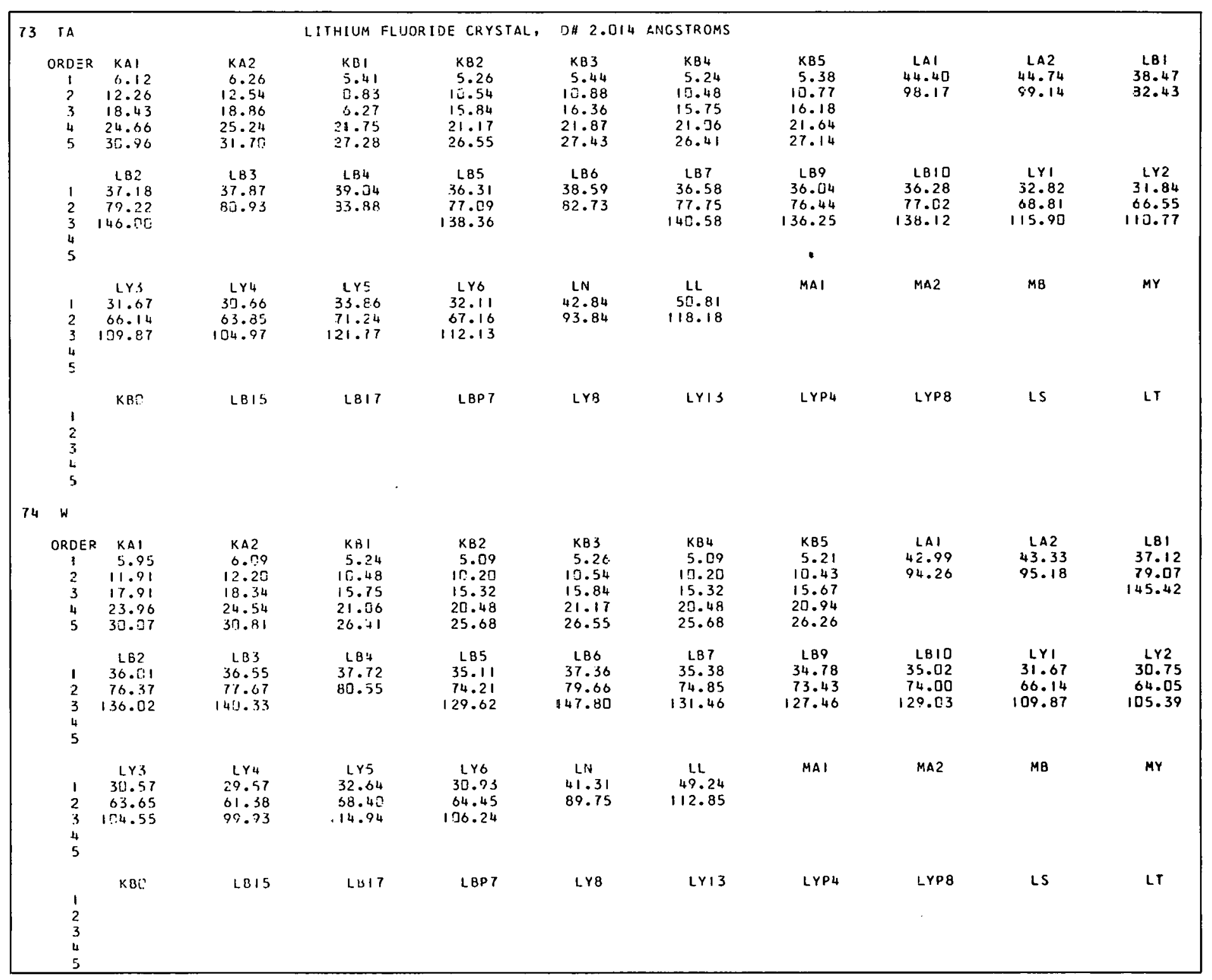




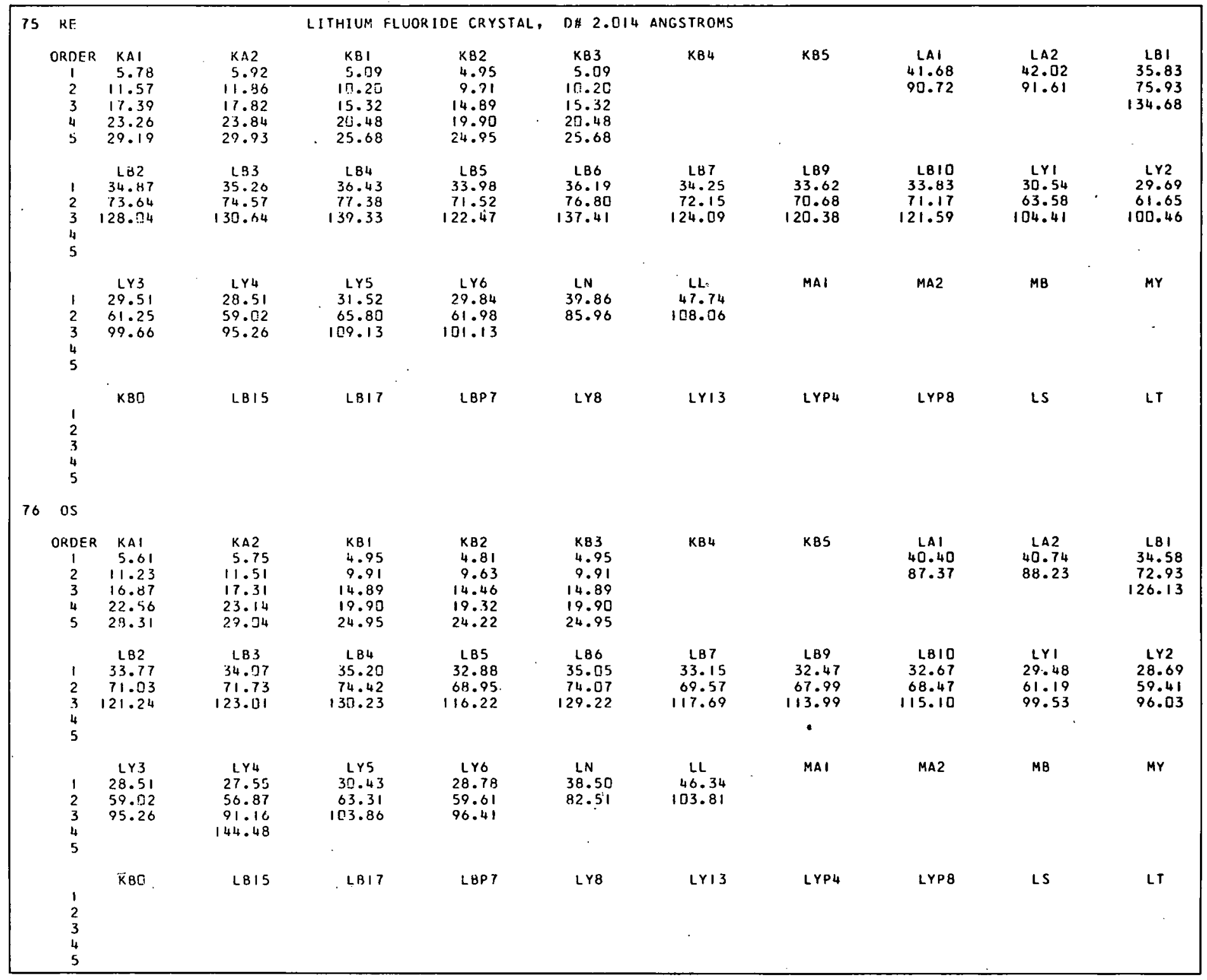




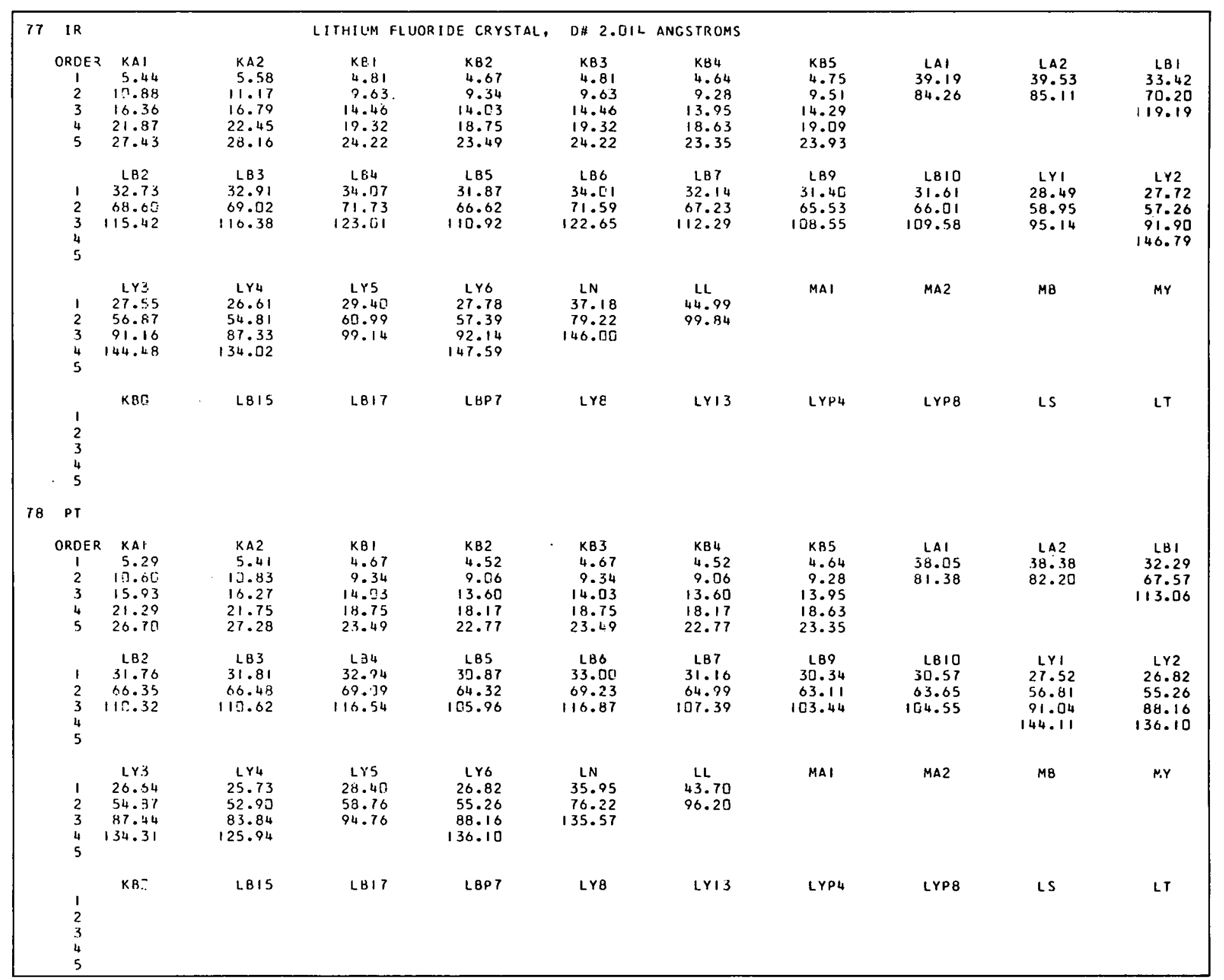




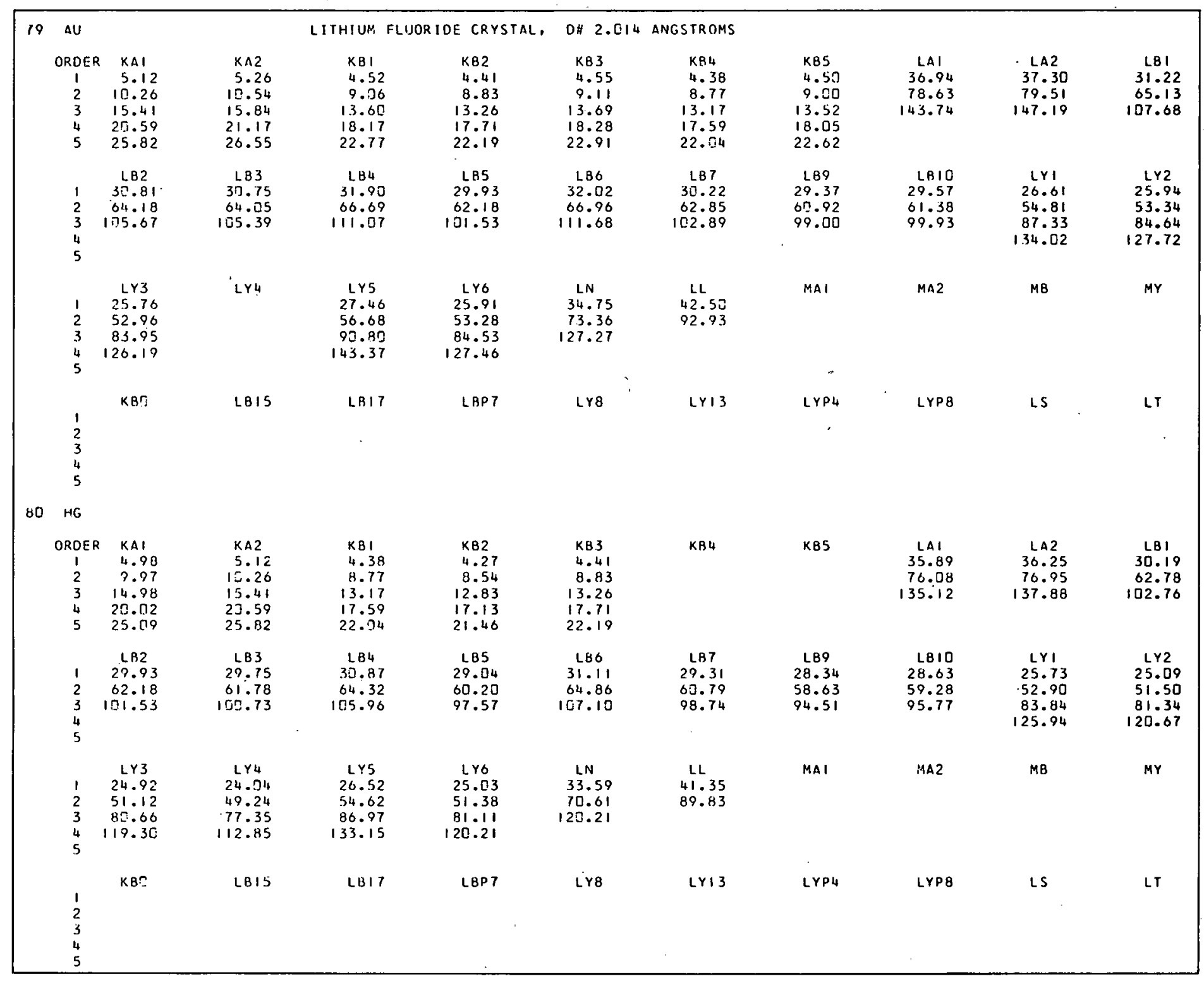




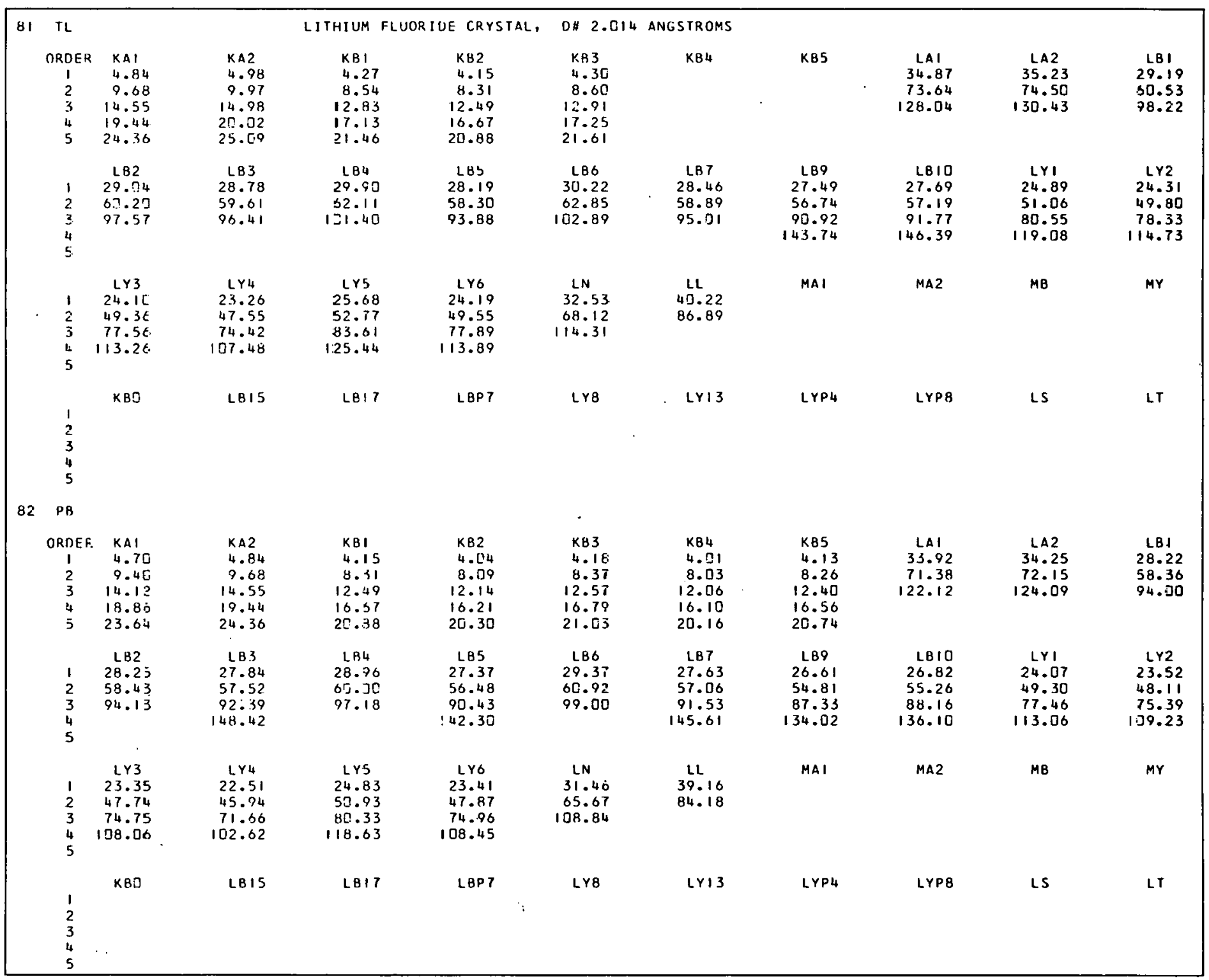




\begin{tabular}{|c|c|c|c|c|c|c|c|c|c|c|c|}
\hline 83 & BI & & & LITHILM & FLIJORIDE CRYSTAL, & D\# 2.514 & ANGSTROMS & & & & \\
\hline & ORDER & KAI & KA2. & K: I & KB2 & K83 & KB4 & KBS & LAI & LA2 & LB I \\
\hline & 1 & 4.58 & 4.72 & 4.54 & 3.73 & 4.07 & & & 33.00 & 33.33 & 27.34 \\
\hline & 2 & 9.17 & 9.46 & 8.09 & 7.86 & 8.14 & & & 69.23 & 69.99 & 56.42 \\
\hline & 3 & 13.77 & 14.25 & 12.14 & 11.30 & 12.23 & & & 116.87 & 118.68 & 90.31 \\
\hline & 4 & 18.40 & 18.98 & 16.21 & 15.75 & 16.3 .3 & & & & & 141.95 \\
\hline & 5 & 23.06 & 23.78 & 20.30 & 19.13 & 27.45 & & & & & \\
\hline & & LB2 & LB.3 & LB4 & LBS & LBO & LB 7 & LBQ & LBID & LYI & LY2 \\
\hline & $\mathbf{I}$ & 27.43 & 26.96 & 28.77 & 26.58 & 29.54 & 26.94 & 25.76 & 25.97 & 23.29 & 22.80 \\
\hline & 2 & 56.61 & 55.58 & 58.04 & 54.75 & 59.08 & 55.32 & 52.96 & 53.40 & 47.62 & 46.56 \\
\hline & 3 & 90.68 & 98.75 & 93.38 & 87.21 & 85.39 & 38.27 & 83.95 & 84.76 & 74.53 & 72.72 \\
\hline & 4 & 143.01 & 137.65 & & 133.72 & & 136.40 & 126.19 & 127.98 & 107.68 & 104.46 \\
\hline & & LY3. & LY4 & LY5 & LYO & LN & LL & MAI & MA2 & $M B$ & MY \\
\hline & 1 & 22.59 & 21.78 & 24.04 & 22.62 & 30.49 & 38.14 & & & & \\
\hline & 2 & 40.13 & 44.40 & 49.24 & 46.19 & $=63.45$ & 81.60 & & & & \\
\hline & 3 & 71.78 & 69.05 & 77.35 & 72.08 & $=104.13$ & & & & & \\
\hline & $\begin{array}{l}4 \\
5\end{array}$ & 103.17 & $\begin{array}{r}98.17 \\
141.69\end{array}$ & 112.85 & 103.35 & & & & & & \\
\hline & & KB? & $\angle B 15$ & $1 B 17$ & LBH 7 & LY8 & LYIS & LYP4 & LYPB & LS & $L T^{\prime}$ \\
\hline & 1 & & & & & & & & & & \\
\hline & $\begin{array}{l}2 \\
3\end{array}$ & & & & & & & & & & \\
\hline & 4 & & & & & & & & & & \\
\hline & 5 & & & & & & & & & & \\
\hline 81 & $4 \quad P O$ & & & & & & & & & & \\
\hline & ORDER & KAI & $\mathrm{KA2}$ & KBI & KB2 & K83 & K84 & KB5 & $\lfloor A \mid$ & LA2 & LBI \\
\hline & 1 & 4.44 & 4.58 & 3.95 & 3.81 & & & & 32.11 & 32.47 & 26.46 \\
\hline & 2 & 8.88 & 9.17 & 7.86 & 7.63 & & & & 67.16 & 67.99 & 54.49 \\
\hline & 3 & 13.34 & 13.77 & 11.80 & 11.46 & & & & 112.13 & 113.99 & 86.74 \\
\hline & 4 & 17.82 & 18.40 & 15.75 & 15.29 & & & & & & 132.58 \\
\hline & 5 & 22.33 & 23.156 & 19.73 & 19.15 & & & & & & \\
\hline & & LB? & LB3 & $\mathrm{LB4}$ & L. B.5 & LB 6 & LB7 & LB9 & LBIO & $\lfloor Y \mid$ & LY2 \\
\hline & 1 & 26.67 & 26.78 & 27.20 & 25.79 & 27.78 & & & & 22.53 & \\
\hline & 2. & 54.94 & 53.66 & 56.10 & 53.02 & 57.39 & & & & 46.00 & \\
\hline & 3 & 87.56 & 85.22 & 89.71 & 84.07 & 92.14 & & & & 71.77 & \\
\hline & 4 & 134.60 & 129.33 & 140.24 & 126.44 & 147.59 & & & & 102.880 & \\
\hline & & & & & & & & & . & & \\
\hline & & $\operatorname{tr} 3$ & $\mathrm{Lr}_{4}$ & LY5 & LYO & LN & LL & MAI & MA2 & M8 & MY \\
\hline & 1 & 21.87 & & & 2.1 .87 & & & & & & \\
\hline & 2 & 44.59 & & & 44.59 & & & & & & \\
\hline & 3 & 67.36 & & & $\begin{array}{r}69.36 \\
\end{array}$ & & & & & & \\
\hline & $\begin{array}{l}4 \\
5\end{array}$ & $\begin{array}{r}99.72 \\
143.71\end{array}$ & & & $\begin{array}{r}98.79 \\
143.91\end{array}$ & & & & & & \\
\hline & & KB: & $\angle B 15$ & LHIT & LBP7 & LYG & $\operatorname{LY} 13$ & LYP4 & LYP 8 & LS & LT \\
\hline & I & & & & & & & & & & \\
\hline & 2 & & . & & & & & & & & \\
\hline & $\frac{3}{4}$ & & & & & & & & & & \\
\hline & 5 & & & & & & & & & & \\
\hline
\end{tabular}




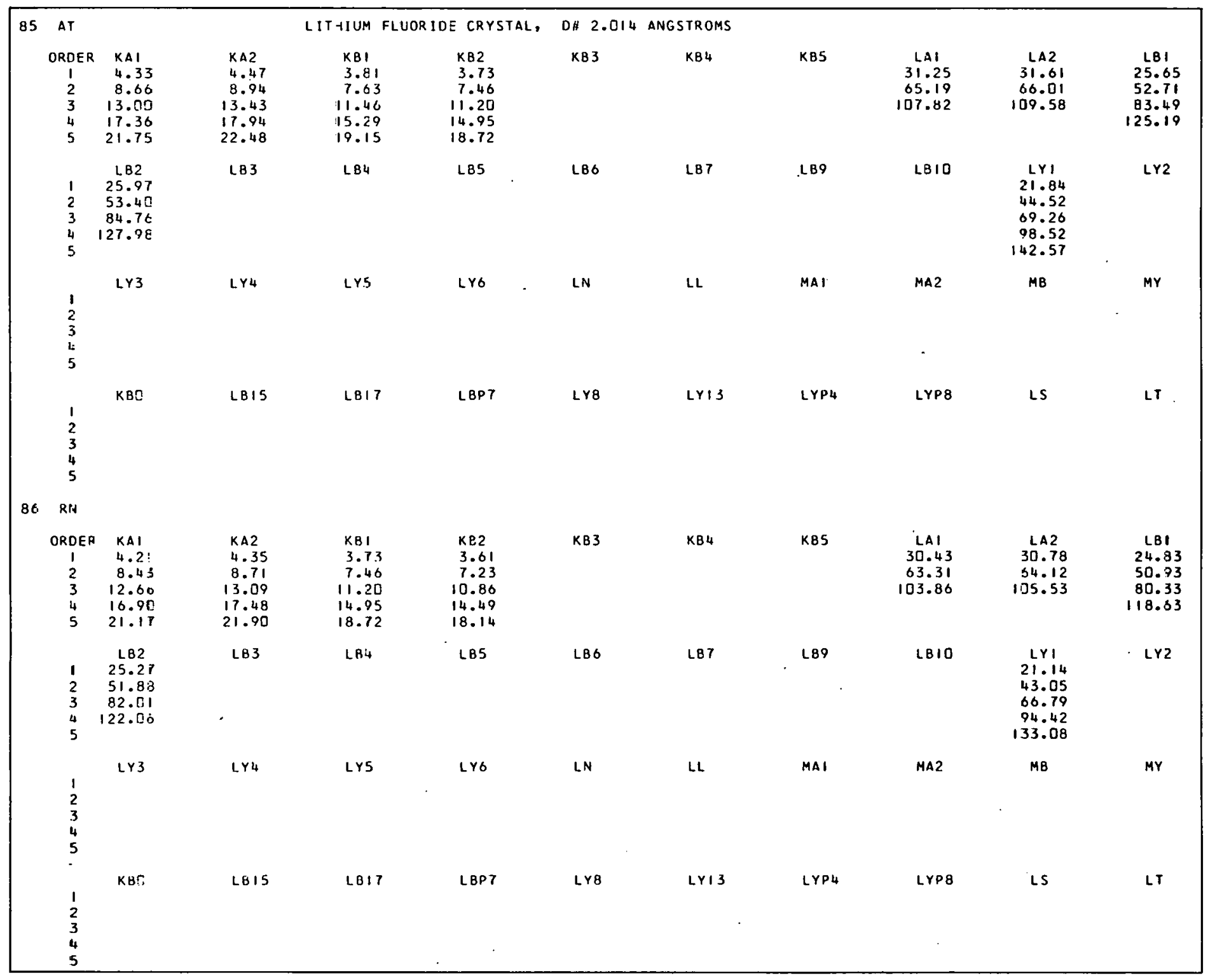




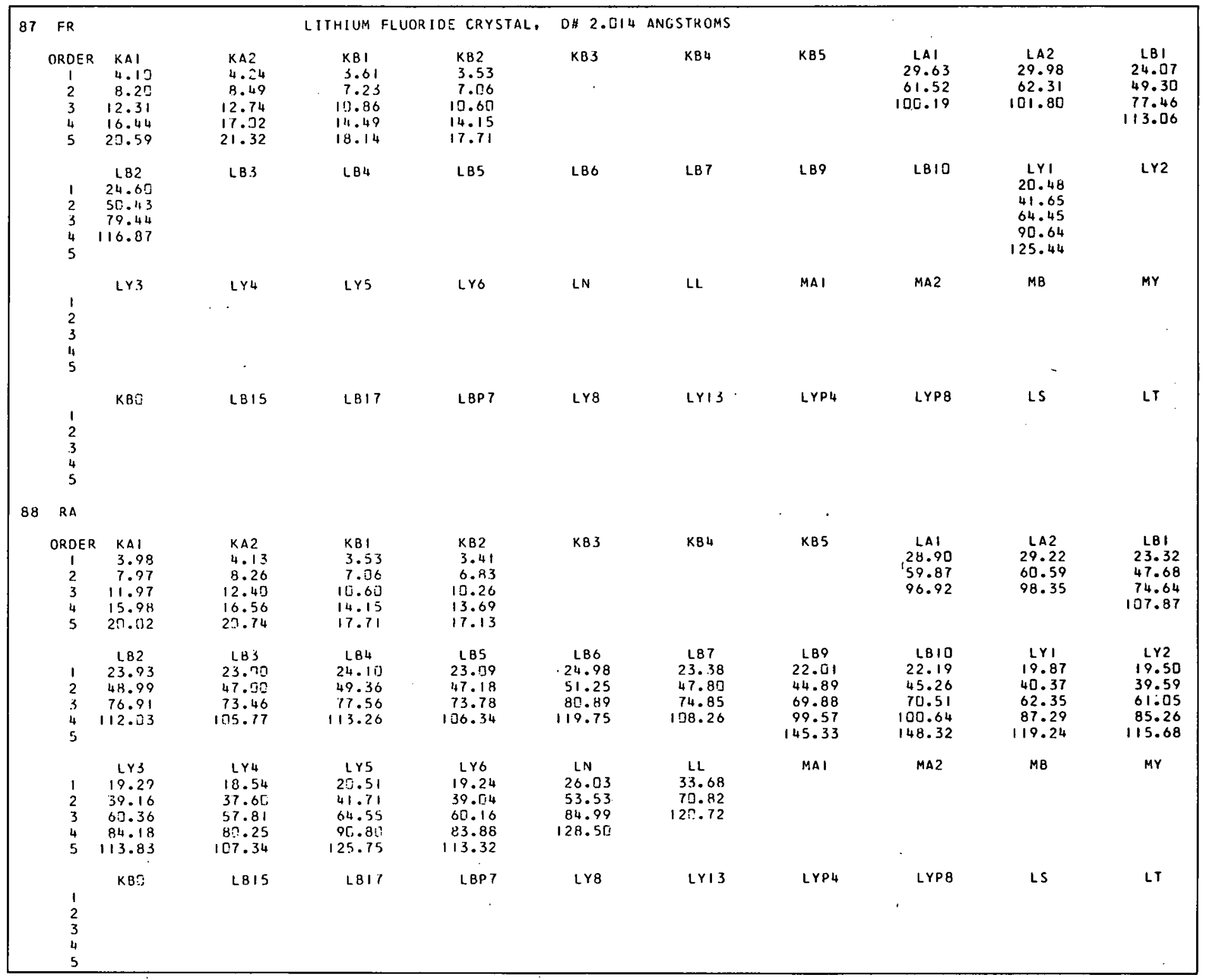




\begin{tabular}{|c|c|c|c|c|c|c|c|c|c|c|c|}
\hline \multirow[t]{5}{*}{87} & $A C$ & & & LITHIUM F & FLUOR IDE CRYSTAL, & D\# 2.014 & ANGSTROMS & & & & \\
\hline & $\begin{array}{c}\text { ORDER } \\
1 \\
2 \\
3 \\
4 \\
5\end{array}$ & $\begin{array}{l}K A 1 \\
3.87 \\
7.74 \\
11.63 \\
15.52 \\
19.44\end{array}$ & $\begin{array}{l}K A 2 \\
4.01 . \\
8.03 \\
12.06 \\
16.10 \\
20.16\end{array}$ & $\begin{array}{l}K B 1 \\
3.44 \\
6.89 \\
10.34 \\
13.83 \\
17.28\end{array}$ & $\begin{array}{l}K B 2 \\
3.33 \\
6.66 \\
10.00 \\
13.34 \\
16.70\end{array}$ & KB3 & KB4 & KBS & $\begin{array}{c}141 \\
23.16 \\
53.23 \\
93.75\end{array}$ & $\begin{array}{l}\text { LA2 } \\
28.51 \\
59.02 \\
95.26\end{array}$ & $\begin{array}{c}281 \\
22.59 \\
46.13 \\
71.98 \\
103.17\end{array}$ \\
\hline & $\begin{array}{l}1 \\
2 \\
3 \\
4 \\
5\end{array}$ & $\begin{array}{c}\text { LB2 } \\
2.3 .37 \\
47.68 \\
74.64 \\
107.87\end{array}$ & L83 & LE.4 & LBS & L86 & $\angle 87$ & LB9 & 2810 & $\begin{array}{l}\lfloor Y \mid \\
19.27 \\
39.10 \\
60.26 \\
84.03 \\
113.57\end{array}$ & LY2 \\
\hline & $\begin{array}{l}1 \\
2 \\
3 \\
4 \\
5\end{array}$ & LY3 & Lru & LY5 & LYo & LN & LL & MAI & MA2 & M8 & MY \\
\hline & $\begin{array}{l}1 \\
2 \\
3 \\
4 \\
5\end{array}$ & KBD & LBI5 & LBI 7 & LBP & LY8 & LYI 3 & LYP4 & LYP8 & LS & LI \\
\hline \multirow[t]{5}{*}{90} & in & & & & & & & & & & \\
\hline & $\begin{array}{c}\text { ORDER } \\
1 \\
2 \\
3 \\
4 \\
5\end{array}$ & $\begin{array}{r}\text { KA1 } \\
3.78 \\
7.57 \\
11.37 \\
15.18 \\
19.01\end{array}$ & $\begin{array}{r}K A 2 \\
3.93 \\
7.86 \\
11.80 \\
15.75 \\
19.73\end{array}$ & $\begin{array}{l}k e 1 \\
3.33 \\
6.06 \\
10.00 \\
13.34 \\
16.70\end{array}$ & $\begin{array}{l}\text { KB2 } \\
3.24 \\
6.49 \\
9.74 \\
13.00 \\
16.27\end{array}$ & $\begin{array}{c}K 83 \\
3.36 \\
6.72 \\
10.08 \\
13.46 \\
16.85\end{array}$ & KB4 & $\begin{array}{l}\text { K85 } \\
3.30 \\
6.60 \\
9.91 \\
13.23 \\
16.56\end{array}$ & $\begin{array}{c}\text { LAl } \\
27.46 \\
56.68 \\
90.80 \\
143.37\end{array}$ & $\begin{array}{c}\text { LA2 } \\
27.81 \\
57.45 \\
92.27 \\
148.00\end{array}$ & $\begin{array}{c}\text { LB1 } \\
21.90 \\
44.65 \\
69.47 \\
98.87 \\
143.46\end{array}$ \\
\hline & $\begin{array}{l}1 \\
2 \\
3 \\
4 \\
5\end{array}$ & $\begin{array}{c}\text { LB2 } \\
22.74 \\
46.44 \\
72.51 \\
104.09\end{array}$ & $\begin{array}{c}183 \\
21.01 \\
114.03 \\
08.43 \\
87.14 \\
139.17\end{array}$ & $\begin{array}{c}134 \\
22.71 \\
46.38 \\
72.40 \\
103.90\end{array}$ & $\begin{array}{r}\text { LB5 } \\
21.90 \\
44.65 \\
69.47 \\
98.87 \\
143.46\end{array}$ & $\begin{array}{c}\text { LB6 } \\
23.72 \\
48.55 \\
76.15 \\
110.62\end{array}$ & $\begin{array}{c}\angle 87 \\
22.16 \\
45.20 \\
70.40 \\
100.46 \\
147.80\end{array}$ & $\begin{array}{c}\text { LB9 } \\
20.68 \\
42.08 \\
65.16 \\
91.77 \\
127.65\end{array}$ & $\begin{array}{r}1810 \\
20.88 \\
42.50 \\
65.87 \\
92.93 \\
129.96\end{array}$ & $\begin{array}{c}\lfloor Y \mid \\
18.66 \\
37.84 \\
58.20 \\
80.85 \\
108.30\end{array}$ & $\begin{array}{r}\text { LY2 } \\
18.34 \\
37.18 \\
57.13 \\
79.22 \\
105.67\end{array}$ \\
\hline & $\begin{array}{l}1 \\
2 \\
3 \\
4 \\
5\end{array}$ & $\begin{array}{r}\operatorname{LY} 3 \\
18.17 \\
36.82 \\
56.55 \\
78.33 \\
104.27\end{array}$ & $\begin{array}{l}2 Y 4 \\
17.45 \\
35.32 \\
54.14 \\
74.71 \\
98.65\end{array}$ & $\begin{array}{r}\text { LYS } \\
19.29 \\
39.16 \\
60.30 \\
84.18 \\
113.83\end{array}$ & $\begin{array}{c}\text { LY6 } \\
18.05 \\
36.58 \\
56.16 \\
77.75 \\
103.35\end{array}$ & $\begin{array}{c}\text { LN } \\
24.48 \\
50.18 \\
79.00 \\
116.00\end{array}$ & $\begin{array}{r}\text { LL } \\
32.14 \\
07.23 \\
112.29\end{array}$ & MAI & MA2 & MB & MY \\
\hline & $\begin{array}{l}1 \\
2 \\
3 \\
4 \\
5\end{array}$ & KBO & 1815 & LBIT & LBPT & LY8 & LYI3 & LYP4 & LYPB & 'LS & LT \\
\hline
\end{tabular}




\begin{tabular}{|c|c|c|c|c|c|c|c|c|c|c|c|}
\hline 9 & $P A$ & & & LITHIUM F & IDE CRYS & $0 \# 2.011$ & ANGSTROMS & & & & \\
\hline \multirow[t]{4}{*}{, } & $\begin{array}{l}\text { ORDER } \\
1 \\
2 \\
3 \\
4 \\
5\end{array}$ & $\begin{array}{l}k A 1 \\
3.67 \\
7.34 \\
11.83 \\
14.72 \\
18.43\end{array}$ & $\begin{array}{l}K A 2 \\
3.81 \\
7.85 \\
11.46 \\
15.29 \\
17.15\end{array}$ & $\begin{array}{l}K B 1 \\
3.24 \\
0.49 \\
9.74 \\
13.90 \\
16.27\end{array}$ & $\begin{array}{l}\text { KB2 } \\
3.16 \\
6.32 \\
9.48 \\
12.68 \\
15.84\end{array}$ & $K B 3$ & K84 & $K B 5$ & $\begin{array}{c}\text { LAI } \\
26.79 \\
55.19 \\
88.04 \\
135.80\end{array}$ & $\begin{array}{c}\text { LA2 } \\
27.14 \\
55.97 \\
89.47 \\
139.58\end{array}$ & $\begin{array}{c}\text { LBI } \\
21.23 \\
43.24 \\
67.10 \\
94.93 \\
134.16\end{array}$ \\
\hline & $\begin{array}{l}1 \\
2 \\
3 \\
4 \\
5\end{array}$ & $\begin{array}{r}\text { LE2 } \\
22.16 \\
45.20 \\
70.40 \\
175.46 \\
147.80\end{array}$ & $\begin{array}{l}\text { LB3 } \\
20.94 \\
42.63 \\
66.07 \\
93.26 \\
130.64\end{array}$ & $\begin{array}{l}\text { LB4 } \\
22.74 \\
44.96 \\
69.99 \\
99.75 \\
145.81\end{array}$ & $\begin{array}{c}\text { LB5 } \\
21.32 \\
43.42 \\
57.40 \\
95.43 \\
135.27\end{array}$ & $\begin{array}{c}L B 6 \\
23.14 \\
47.31 \\
74.00 \\
156.72\end{array}$ & $\begin{array}{c}2 B 7 \\
21.01 \\
44.03 \\
68.43 \\
97.14 \\
139.17\end{array}$ & $\begin{array}{c}\text { L89 } \\
20.04 \\
40.74 \\
62.95 \\
88.23 \\
120.95\end{array}$ & $\begin{array}{r}2810 \\
20.25 \\
41.16 \\
63.65 \\
89.35 \\
123.01\end{array}$ & $\begin{array}{l}\lfloor Y \mid \\
18.11 \\
36.70 \\
56.35 \\
78.04 \\
103.81\end{array}$ & $\begin{array}{l}\text { LY2 } \\
17.82 \\
36.10 \\
55.39 \\
76.58 \\
101.53\end{array}$ \\
\hline & $\begin{array}{l}1 \\
2 \\
3 \\
4 \\
5\end{array}$ & $\begin{array}{l}\text { LY3 } \\
17.62 \\
35.68 \\
54.71 \\
75.57 \\
99.97\end{array}$ & $\begin{array}{l}L Y 4 \\
16.96 \\
34.31 \\
52.51 \\
72.30 \\
95.71\end{array}$ & $\begin{array}{l}\text { LY5 } \\
18.72 \\
37.96 \\
58.45 \\
81.15 \\
108.79\end{array}$ & $\begin{array}{l}\text { LY6 } \\
17.51 \\
35.44 \\
54.33 \\
85.00 \\
99.59\end{array}$ & $\begin{array}{l}\text { LN } \\
23.75 \\
48.61 \\
76.26 \\
110.82\end{array}$ & $\begin{array}{c}\text { LL } \\
31.43 \\
65.60 \\
108.69\end{array}$ & MAI & . & $M B$ & MY \\
\hline & $\begin{array}{l}1 \\
2 \\
3 \\
4 \\
5\end{array}$ & $\mathrm{KBr}$ & LBI5 & LBI7 & $\angle B P T$ & $\operatorname{Lr} 8^{\circ}$ & LYI3 & LYP4 & $\operatorname{LYP} 8$ & is & LT \\
\hline \multirow[t]{3}{*}{92} & $\mathrm{u}$ & & & & & & & & & & \\
\hline & $\begin{array}{c}\text { ORDER } \\
1 \\
2 \\
3 \\
4 \\
5\end{array}$ & $\begin{array}{l}K A 1 \\
3.59 \\
7.17 \\
1.7 .77 \\
14.38 \\
18.57\end{array}$ & $\begin{array}{l}K A 2 \\
3.73 \\
7.46 \\
11.26 \\
14.95 \\
18.72\end{array}$ & $\begin{array}{l}K B 1 \\
3.16 \\
6.32 \\
7.48 \\
12.66 \\
15.84\end{array}$ & $\begin{array}{l}k 82 \\
3.10 \\
0.20 \\
9.31 \\
12.43 \\
15.55\end{array}$ & $\begin{array}{c}K B 3 \\
3.19 \\
6.38 \\
9.57 \\
12.77 \\
15.78\end{array}$ & KB 4 & K85 & $\begin{array}{c}\text { LA1 } \\
26.14 \\
53.79 \\
85.45 \\
129.56\end{array}$ & $\begin{array}{c}\text { LA2 } \\
26.46 \\
54.49 \\
86.74 \\
132.58\end{array}$ & $\begin{array}{r}\text { LB1 } \\
20.59 \\
41.89 \\
64.86 \\
91.29 \\
126.70\end{array}$ \\
\hline & $\begin{array}{l}1 \\
2 \\
3 \\
4 \\
5\end{array}$ & $\begin{array}{r}\text { LB2 } \\
21.61 \\
44.73 \\
68.43 \\
97.14 \\
1.39 .17\end{array}$ & $\begin{array}{c}183 \\
20.30 \\
41.28 \\
63.85 \\
89.67 \\
123.61\end{array}$ & $\begin{array}{l}\text { LB4 } \\
21.4 C \\
43.60 \\
07.71 \\
95.94 \\
136.40\end{array}$ & $\begin{array}{l}\text { LB5 } \\
20.77 \\
42.26 \\
65.46 \\
92.27 \\
1.28 .63\end{array}$ & $\begin{array}{c}\text { LB6 } \\
22.56 \\
46.07 \\
71.87 \\
102.98 \\
.\end{array}$ & $\begin{array}{c}287 \\
21.06 \\
42.87 \\
66.48 \\
93.92 \\
132.02\end{array}$ & $\begin{array}{c}\text { LB9 } \\
19.47 \\
39.53 \\
60.95 \\
85.11 \\
115.42\end{array}$ & $\begin{array}{r}2810 \\
19.64 \\
39.89 \\
61.55 \\
86.04 \\
117.03\end{array}$ & $\begin{array}{c}L Y 1 \\
17.56 \\
35.56 \\
54.52 \\
75.28 \\
99.53\end{array}$ & $\begin{array}{l}\text { LY2 } \\
17.28 \\
34.96 \\
53.56 \\
73.85 \\
97.35\end{array}$ \\
\hline
\end{tabular}




\begin{tabular}{|c|c|c|c|c|c|c|c|c|c|c|c|}
\hline \multirow[t]{5}{*}{93} & $N P$ & & & LI-HIUM & FLUORIDE CRYSTAL, & D\# 2.014 & ANGSTROMS & & & & \\
\hline & $\begin{array}{c}\text { ORDER } \\
1 \\
2 \\
3 \\
4 \\
5\end{array}$ & $\begin{array}{c}K A 1 \\
3.50 \\
7.00 \\
19.51 \\
14.03 \\
17.56\end{array}$ & $\begin{array}{l}K A 2 \\
3.64 \\
7.29 \\
1.3 .94 \\
14.61 \\
18.28\end{array}$ & $\begin{array}{c}K 81 \\
3.10 \\
6.20 \\
7.31 \\
12.43 \\
15.55\end{array}$ & $\begin{array}{c}K B 2 \\
2.99 \\
5.98 \\
8.97 \\
11.97 \\
14.98\end{array}$ & KB3 & KB4 & KB5 & $\begin{array}{c}\text { LAl } \\
25.50 \\
52.39 \\
82.92 \\
123.97\end{array}$ & $\begin{array}{r}\text { LA2 } \\
25.85 \\
53.15 \\
84.30 \\
126.95\end{array}$ & $\begin{array}{r}181 \\
19.96 \\
40.56 \\
62.65 \\
87.76 \\
120.09\end{array}$ \\
\hline & $\begin{array}{l}1 \\
2 \\
3 \\
4 \\
5\end{array}$ & $\begin{array}{c}\text { LB2 } \\
21.96 \\
42.87 \\
66.48 \\
93.92 \\
132.72\end{array}$ & LB3 & $\mathrm{Le}_{4}$ & L85 & LB6 & LBT & L89 & LBIO & $\begin{array}{l}\text { LYI } \\
17.05 \\
34.49 \\
52.80 \\
72.72 \\
95.64\end{array}$ & LY2 \\
\hline & $\begin{array}{l}1 \\
2 \\
3 \\
4 \\
5\end{array}$ & LY3 & LY4 & LYS & LYo & LN & $L L$ & MAI & MA2 & M8 & MY \\
\hline & $\begin{array}{l}1 \\
2 \\
3 \\
4 \\
5\end{array}$ & KBO & LBIS & $\lfloor B \mid 7$ & LBP 7 & LY8 & LY13 & LYPH & LYP8 & LS & LT \\
\hline \multirow[t]{5}{*}{94} & $4 \mathrm{PU}$ & & & & & & & & & 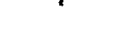 & \\
\hline & $\begin{array}{c}\text { ORDER } \\
1 \\
2 \\
3 \\
4 \\
5\end{array}$ & $\begin{array}{r}\text { RA1 } \\
3.41 \\
6.83 \\
10.26 \\
13.69 \\
17.13\end{array}$ & $\begin{array}{l}K A 2 \\
3.56 \\
7.12 \\
10.68 \\
14.26 \\
17.85\end{array}$ & $\begin{array}{r}K B 1 \\
3.02 \\
6.03 \\
9.06 \\
12.08 \\
15.12\end{array}$ & $\begin{array}{l}K B 2 \\
2.93 \\
5.86 \\
8.80 \\
11.74 \\
14.69\end{array}$ & KB3 & KंB 4 & KB5 & $\begin{array}{c}\text { LA1 } \\
24.89 \\
51.06 \\
80.55 \\
119.08\end{array}$ & $\begin{array}{r}\text { LA2 } \\
25.24 \\
51.82 \\
81.90 \\
121.83\end{array}$ & $\begin{array}{r}181 \\
19.38 \\
39.35 \\
60.66 \\
84.64 \\
114.62\end{array}$ \\
\hline & $\begin{array}{l}1 \\
2 \\
3 \\
4 \\
5\end{array}$ & $\begin{array}{l}\text { LA2 } \\
20.54 \\
41.77 \\
64.65 \\
90.96 \\
126.06\end{array}$ & LB3 & LB4 & LA5 & L86 & LB? & L89 & LB1O & $\begin{array}{l}L Y 1 \\
16.53 \\
33.42 \\
51.09 \\
70.20 \\
91.90\end{array}$ & LY2 \\
\hline & $\begin{array}{l}1 \\
2 \\
3 \\
4 \\
5\end{array}$ & LY3 & $\mathrm{Lr}_{4}$ & LY5 & LYo & $2 \mathrm{~N}$ & LL & MAI & MA2 & MB & MY \\
\hline & $\begin{array}{l}1 \\
2 \\
3 \\
4 \\
5\end{array}$ & KBo & L815 & LBI? & LBP 7 & LYB & LYIJ & LYP4 & LYPB & LS & LT \\
\hline
\end{tabular}




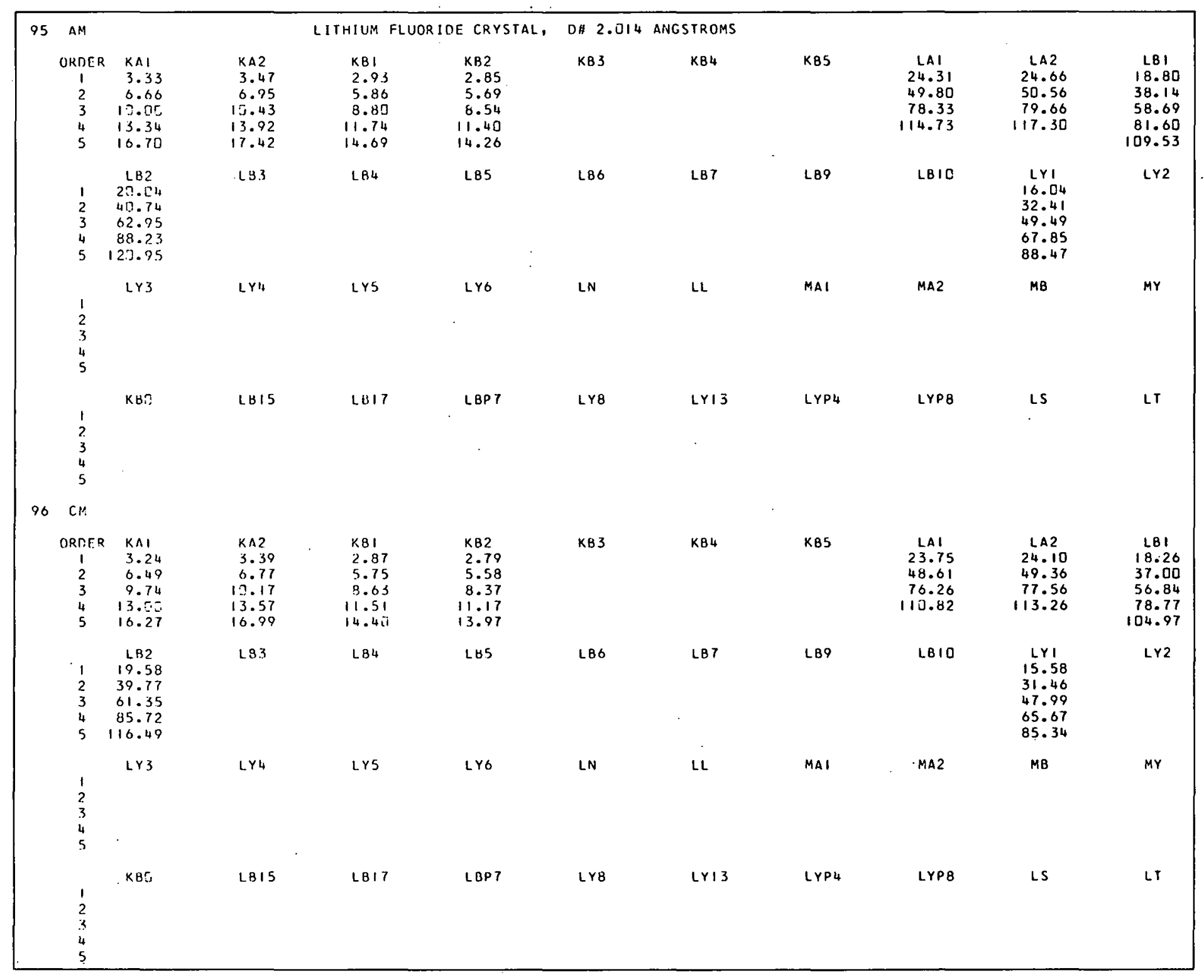




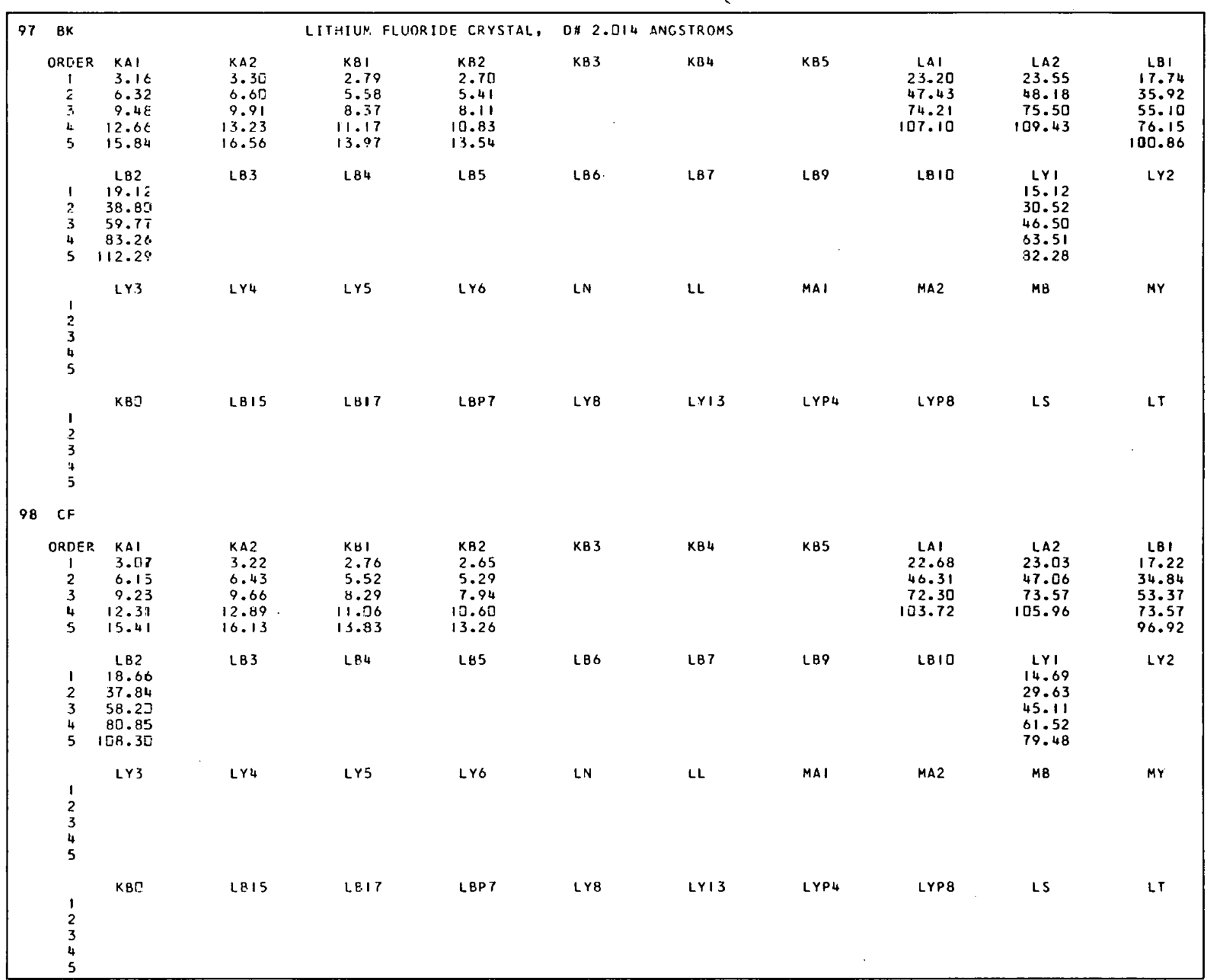


‘

THIS PAGE

WAS INTENTIONALLY

LEFT BLANK 
TABLE 2

GONIOMETER ANGLES ARRANGED BY ANGLE MAGNITUDE 


\section{THIS PAGE}

\section{WAS INTENTIONALLY \\ LEFT BLANK}




\begin{tabular}{|c|c|c|c|c|c|c|c|c|c|c|}
\hline & & LITH & IUM & FLUOR $1:)$ & E CRYSTAL, D\#2.014 A & ANGSTROMS & & & 2.6510 & 3.87 \\
\hline ANCLE & ELEM & LINE & $N$ & LAMBDA & $R \in F$ & ANGLE & ELEM & LINE & $N$ LAMBDA & REF \\
\hline 2.65 & $98 \mathrm{CF}$ & KB2 & 1 & 0.1 .93 & 1 & 3.33 & $95 \Delta M$ & KAI & 0.117 & 1 \\
\hline 2.70 & $97 \mathrm{BK}$ & $\mathrm{KB2}$ & 1 & 0.095 & 1 & 3.33 & $89 \mathrm{AC}$ & KB2 & 0.117 & 1 \\
\hline 2.76 & $78 \mathrm{CF}$ & KBI & 1 & C. 597 & 1 & 3.36 & $90 \mathrm{TH}$ & KB3 & 0.118 & 1.2 \\
\hline 2.79 & $96 \mathrm{CM}$ & KB2 & 1 & 0.098 & 1 & 3.39 & $96 \mathrm{CM}$ & $\mathrm{KA2}$ & 0.119 & $\mathbf{1}$ \\
\hline 2.79 & $97 \mathrm{BK}$ & KB। & 1 & 0.098 & 1 & 3.41 & $94 \mathrm{PU}$ & KAI & 0.120 & $\mathbf{1}$ \\
\hline 2.84 & 95 AM & kB2 & । & 0.100 & 1 & 3.41 & $88 \mathrm{RA}$ & KB2 & 0.120 & 1 \\
\hline 2.87 & $96 \mathrm{~cm}$ & KuI & 1 & 0.101 & 1 & 3.44 & $89 \mathrm{AC}$ & KBI & 0.121 & 1 \\
\hline 2.93 & $95 \Delta M$ & $K B I$ & 1 & 0.173 & 1 & 3.47 & $95 \mathrm{AM}$ & KA2 & 0.122 & 1 \\
\hline 2.93 & $94 \mathrm{PU}$ & $K B 2$ & 1 & 0.103 & 1 & 3.50 & $33 \mathrm{NP}$ & KAI & 0.123 & 1 \\
\hline 2.99 & $93 \mathrm{NP}$ & $\leqslant \mathrm{B} 2$ & 1 & 0.105 & 1 & 3.53 & $87 \mathrm{FR}$ & $K B Z$ & 0.124 & 1 \\
\hline 3.02 & $94 \mathrm{PU}$ & KBI & 1 & 0.106 & 1 & 3.53 & $88 \mathrm{RA}$ & KB I & 0.124 & 1 \\
\hline 3.07 & $98 \mathrm{CF}$ & KAI & 1 & 0.108 & 1 & 3.56 & $94 \mathrm{PU}$ & KA2 & 0.125 & 1 \\
\hline 3.10 & $92 \mathrm{U}$ & $K 82$ & 1 & 0.109 & $1,2,3$ & 3.58 & $92 \mathrm{U}$ & KAI & 0.126 & $1,2,3$ \\
\hline 3.15 & $93 \mathrm{NP}$ & KBI & 1 & 0.108 & 1 & 3.61 & $87 \mathrm{FR}$ & KBI & 0.127 & 1 \\
\hline 3.16 & $92 \mathrm{U}$ & KB'I & 1 & 0.111 & $1,2,3$ & 3.61 & $86 \mathrm{RN}$ & $\mathrm{KB2}$ & 0.127 & 1 \\
\hline 3.16 & $91 \mathrm{PA}$ & KB2 & 1 & 0.111 & 1 & 3.64 & $93 \mathrm{NP}$ & KA2 & 0.128 & 1 \\
\hline 3.16 & $97 \mathrm{BK}$ & KAI & 1 & 0.111 & 1 & 3.67 & $91 \mathrm{PA}$ & KAI & D. 129 & 1 \\
\hline 3.19 & $92 \mathrm{U}$ & $K B 3$ & 1 & 0.112 & 1,2 & 3.73 & $86 \mathrm{RN}$ & KBI & 0.131 & 1 \\
\hline 3.21 & $98 \mathrm{CF}$ & KA2 & 1 & 0.113 & 1 & 3.73 & $92 \mathrm{U}$ & KA2 & 0.131 & $1,2,3$ \\
\hline 3.24 & $90 \mathrm{TH}$ & $K B 2$ & 1 & 0.114 & 1,3 & 3.73 & BS AT & KB2 & 0.131 & 1 \\
\hline 3.24 & $91 P A$ & KBI & 1 & 0.114 & 1 & 3.78 & $90 \mathrm{TH}$ & KA 1 & 0.133 & $1,2,3$ \\
\hline 3.24 & $96 \mathrm{~cm}$ & KAI & 1 & 0.114 & 1 & 3.81 & $91 \mathrm{PA}$ & KA2 & 0.134 & 1 \\
\hline 3.30 & $97 \mathrm{BK}$ & KAZ & 1 & 0.116 & 1 & 3.81 & $84 \mathrm{PO}$ & KB2 & 0.134 & 1 \\
\hline 3.30 & $90 \mathrm{TH}$ & KB5 & 1 & 0.116 & 1,2 & 3.81 & 85 AT & KBI & 0.134 & 1 \\
\hline 3.33 & $90 \mathrm{rH}$ & KBI & 1 & 0.117 & $1,2,3$ & 3.87 & $89 A C$ & KAI & 0.136 & 1 \\
\hline
\end{tabular}




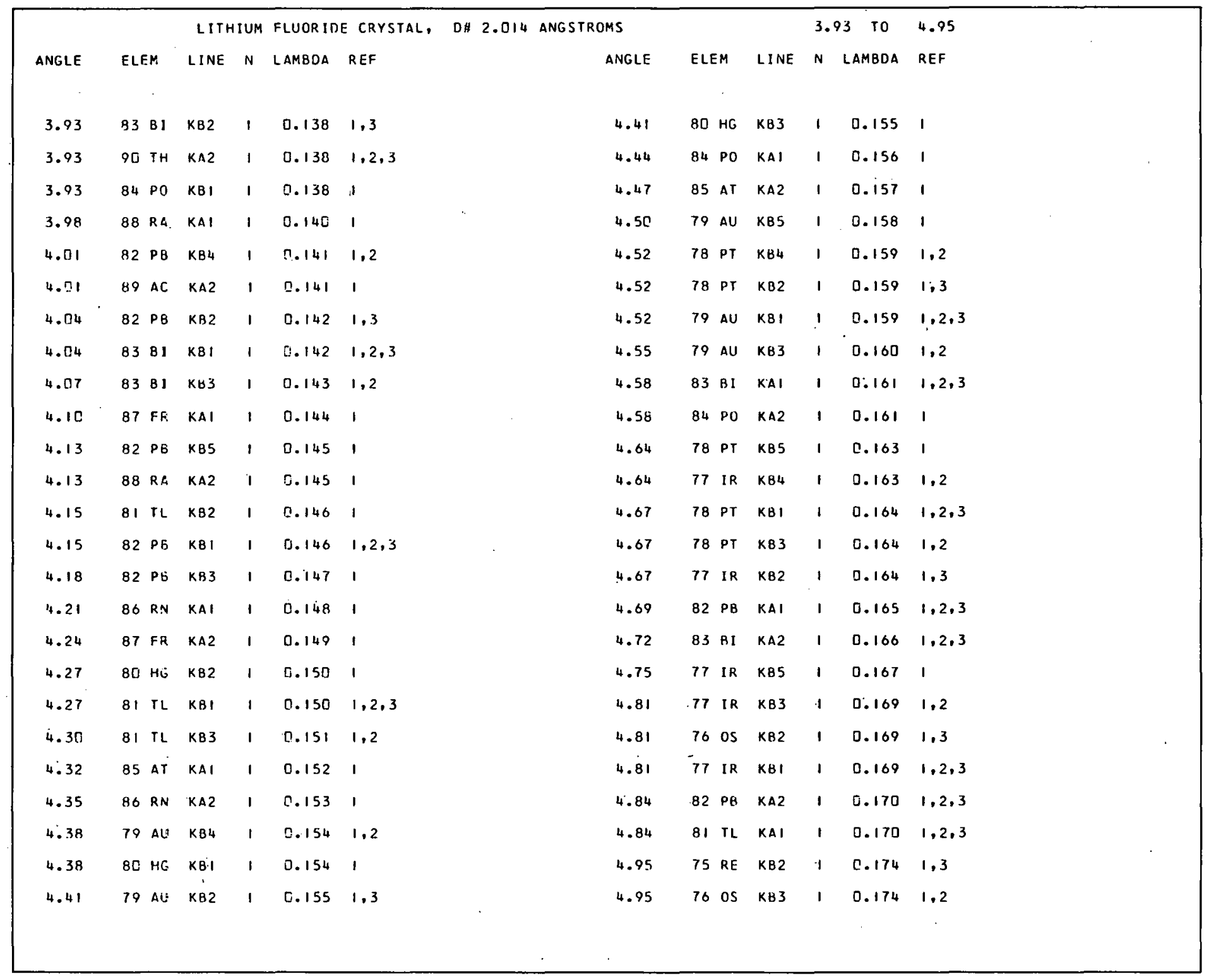




\begin{tabular}{|c|c|c|c|c|c|c|c|c|c|c|c|c|c|}
\hline \multirow[b]{2}{*}{ ANGLE } & \multirow{2}{*}{\multicolumn{2}{|c|}{ ELEM }} & \multicolumn{2}{|c|}{ LITHIUM } & \multirow{2}{*}{$\begin{array}{l}\text { FLUOR IDE } \\
\text { LAMBOA. }\end{array}$} & \multirow{2}{*}{$\begin{array}{l}\text { E CRYSTAL, } \\
\text { REF }\end{array}$} & \multirow[t]{2}{*}{ DH 2.014} & \multicolumn{3}{|l|}{ ANGSTROMS } & \multicolumn{2}{|c|}{4.95 т0 } & \multirow{2}{*}{$\begin{array}{l}6.12 \\
\text { REF }\end{array}$} \\
\hline & & & LINE & N & & & & ANGLE & ELEM & LINE & $\mathrm{N}$ & LAMBDA & \\
\hline 4.95 & 76 & os & $K B:$ & I & .0 .174 & $1,2,3$ & & 5.56 & $72 \mathrm{HF}$ & $K 83$ & 1 & 0.196 & 1,2 \\
\hline 4.98 & 81 & TL & KA2 & 1 & r. 175 & $1,2,3$ & & 5.58 & $72 \mathrm{HF}$ & KBI & 1 & 0.196 & $1,2,3$ \\
\hline 4.99 & $8 E$ & HG & KAI & 1 & 5.175 & 1 & & 5.58 & $77 \mathrm{IR}$ & KA2 & 1 & 0.196 & $1,2,3$ \\
\hline 5.09 & 74 & $w$ & $\mathrm{~KB} 4$ & 1 & 0.179 & 1 & & 5.56 & $96 \mathrm{CM}$ & KBZ & 2 & 0.098 & 1 \\
\hline 5.09 & 75 & $R E$ & $K 83$ & 1 & C.175 & 1,2 & & 5.58 & $97 \mathrm{BK}$ & KBI & 2 & 0.098 & 1 \\
\hline 5.19 & 75 & RE & $\mathrm{KBI}$ & 1 & 0.175 & $1,2,3$ & & 5.31 & 76 os & KAI & 1 & 0.197 & $1,2,3$ \\
\hline 5.09 & 74 & $w$ & KB2 & 1 & 0.175 & $1,2,3$ & & 5.31 & $71 \mathrm{LU}$ & Ke 2 & 1 & 0.197 & $1,2,3$ \\
\hline 5.12 & 79 & $A U$ & KAI & 1 & $0.18 c$ & $1,2,3$ & & 5.69 & 95 AM & KE.2 & 2 & 0.100 & $\mathbf{I}$ \\
\hline 5.12 & 80 & HG & KA2 & 1 & 0.180 & 1 & & 5.75 & 76 OS & $K A 2$ & 1 & 0.202 & $1,2,3$ \\
\hline 5.21 & 74 & $w$ & KB5 & 1 & 0.183 & 1 & & 5.75 & $71 \mathrm{LU}$ & KEI & 1 & 0.202 & $1,2,3$ \\
\hline 5.24 & 73 & TA & $K B 4$ & 1 & 0.182 & 1 & & 5.75 & $96 \mathrm{~cm}$ & KBI & 2 & 0.101 & 1 \\
\hline 5.24 & 74 & $w$ & KBI & 1 & 0.184 & $1,2,3$ & & 5.78 & $75 \mathrm{RE}$ & $\mathrm{K} 4 \mathrm{I}$ & 1 & 0.203 & $1,2,3$ \\
\hline 5.26 & 73 & TA & K82 & 1 & 0.185 & $1,2,3$ & & 5.78 & $71 \mathrm{LU}$ & $K B 3$ & 1 & 0.203 & 1,2 \\
\hline 5.26 & 74 & $w$ & KB 3 & 1 & 0.185 & 1,2 & & 5.81 & $70 \mathrm{YB}$ & KB2 & 1 & 0.204 & $1,2,3$ \\
\hline 5.26 & 79 & $A U$ & $K A 2$ & 1 & 0.185 & $1,2,3$ & & 5.86 & $95 \mathrm{AM}$ & KBI & 2 & 0.103 & 1 \\
\hline 5.29 & 98 & $C F$ & KB2 & 2 & 0.093 & 1 & & 5.86 & 94 PII & KB2 & 2 & 0.103 & 1 \\
\hline 5.29 & 78 & PT & KAI & 1 & $? .18 .5$ & $1,2,3$ & & 5.92 & $75 \mathrm{RE}$ & KA2 & 1 & 0.208 & $1,2,3$ \\
\hline 5.38 & 73 & $T A$ & KBS & 1 & 0.187 & 1 & & 5.95 & $70 \mathrm{YB}$ & $K B I$ & 1 & 0.208 & $1,2,3$ \\
\hline 5.41 & 97 & $B K$ & $\mathrm{KB2}$ & 2 & 0.0 .95 & 1 & & 5.95 & $70 Y 8$ & KB3 & 1 & 0.209 & 1,2 \\
\hline 5.41 & 78 & $P_{T}$ & $K \Delta 2$ & 1 & 0.190 & $1,2,3$ & & 5.95 & $74 \mathrm{~W}$ & KAI & 1 & 0.209 & $1,2,3$ \\
\hline 5.41 & 73 & $T A$ & KEI & 1 & $0.19 \mathrm{~J}$ & $1,2,3$ & & 5.98 & $69 \mathrm{TM}$ & $k 32$ & 1 & 0.210 & 1 \\
\hline 5.44 & 72 & $\mathrm{HF}$ & $k: 2$ & 1 & 0.191 & $1,2,3$ & & 5.98 & $93 \mathrm{NP}$ & $\kappa 32$ & 2 & 0.105 & 1 \\
\hline 5.44 & 77 & IR & $K . .1$ & 1 & 0.191 & $1,2,3$ & & 6.03 & $94 \mathrm{PU}$ & KBI & 2 & 0.106 & 1 \\
\hline 5.44 & 73 & rA & KB 3 & 1 & 0.191 & 1,2 & & 0.09 & $74 \mathrm{~W}$ & KA2 & 1 & 0.214 & $1,2,3$ \\
\hline 5.52 & 98 & $C F$ & KBI & 2 & 0.097 & 1 & & 0.12 & $73 \mathrm{TA}$ & KAI & 1 & 0.215 & $1,2,3$ \\
\hline
\end{tabular}




\begin{tabular}{|c|c|c|c|c|c|c|c|c|c|c|c|c|c|}
\hline \multirow[b]{2}{*}{ ANGLE } & \multirow[b]{2}{*}{ ELEM } & \multicolumn{2}{|c|}{ LITHIUM } & \multirow{2}{*}{$\begin{array}{l}\text { FLUOR IDE } \\
\text { LAMBDA }\end{array}$} & \multirow{2}{*}{$\begin{array}{l}\text { E CRYSTAL, } \\
\text { REF }\end{array}$} & \multirow[t]{2}{*}{$0 \$ 2.014$} & \multicolumn{4}{|l|}{ ANGSTROMS } & \multicolumn{2}{|c|}{$6.12 \mathrm{T0}$} & \multirow{2}{*}{$\begin{array}{l}7.17 \\
\text { REF }\end{array}$} \\
\hline & & LINE & $N$ & & & & ANGLE & ELE & & LINE & $\mathrm{N}$ & LAMBDA & \\
\hline 6.12 & $69 \mathrm{TM}$ & KBi & 1 & 0.215 & $1,2,3$ & & 6.66 & 90 & $T H$ & $K B 1$ & 2 & 0.117 & $1,2,3$ \\
\hline 6.15 & $98 \mathrm{CF}$ & KAI & 2 & 0.108 & 1 & & 6.66 & 95 & $A M$ & KAI & 2 & 0.117 & 1 \\
\hline 6.15 & $69 \mathrm{TM}$ & KB3 & 1 & 0.216 & 1,2 & & 6.66 & 71 & LU & KA2 & 1 & 0.234 & $1,2,3$ \\
\hline 6.18 & $68 \mathrm{ER}$ & K甘2 & 1 & 0.217 & $1,2,3$ & & 0.66 & 89 & $A C$ & $K B 2$ & 2 & 0.117 & 1 \\
\hline $6.2 \pi$ & $92 \mathrm{U}$ & $K B 2$ & 2 & 0.109 & $1,2,3$ & & 6.72 & 90 & $T H$ & $K B 3$ & 2 & 0.118 & 1,2 \\
\hline 6.20 & $93 \mathrm{NP}$ & $K 81$ & 2 & ธ. 109 & 1 & & 6.75 & 66 & DY & KBI & 1 & 0.237 & $1,2,3$ \\
\hline $6.2 \mathrm{~h}$ & $73 \mathrm{TA}$ & KA2 & 1 & 0.220 & $1,2,3$ & & 0.75 & 70 & $Y_{B}$ & KAI & 1 & 0.237 & $1,2,3$ \\
\hline 6.32 & $92 \mathrm{U}$ & KBI & 2 & 9.111 & $1,2,3$ & & 0.77 & 66 & DY & $\mathrm{KB3}$ & 1 & 0.238 & 1,2 \\
\hline 6.32 & $72 \mathrm{HF}$ & KAI & 1 & 0.222 & $1,2,3$ & & 6.77 & 96 & $C M$ & KA2 & 2 & 0.119 & 1 \\
\hline 6.32 & $91 \mathrm{PA}$ & KB2 & 2 & 0.111 & 1 & & 6.83 & 88 & RA & $K B 2$ & 2 & 0.120 & 1 \\
\hline 6.32 & $978 \mathrm{k}$ & KAI & 2 & 0.111 & 1 & & 6.83 & 94 & PU & KAI & 2 & 0.120 & 1 \\
\hline 6.35 & $68 \mathrm{ER}$ & $K B I$ & 1 & 0.223 & $1,2,3$ & & 0.83 & 65 & TB & KB2 & 1 & 0.240 & $1,2,3$ \\
\hline 6.35 & O४ ER. & $K 83$ & 1 & 0.223 & 1,2 & & 6.86 & 70 & YB & KA2 & 1 & 0.241 & $1,2,3$ \\
\hline 6.38 & $92 \mathrm{U}$ & KB 3 & 2 & 0.112 & 1,2 & & 0.89 & 89 & $A C$ & KB! & 2 & 0.121 & $i$ \\
\hline 6.38 & $67 \mathrm{HC}$ & KB2 & 1 & 0.224 & 1 & & 6.95 & 95 & $A M$ & KA2 & 2 & 0.122 & 1 \\
\hline 6.43 & $98 \mathrm{CF}$ & KA2 & 2 & 0.113 & 1 & & 6.95 & 69 & $T M$ & KAI & 1 & 0.244 & $1,2,3$ \\
\hline 6.46 & $72 \mathrm{HF}$ & KA2 & 1 & 0.227 & $1,2,3$ & & 7.00 & 65 & TB & KB3 & 1 & 0.246 & 1,2 \\
\hline 6.49 & $90 \mathrm{TH}$ & KB2 & 2 & 0.1114 & 1,3 & & 7.00 & 93 & NP & KAI & 2 & 0.123 & 1 \\
\hline 6.49 & $96 \mathrm{Cin}$ & KAI & 2 & 0.114 & 1 & & 7.00 & 65 & TB & KEI & 1 & 0.246 & $1,2,3$ \\
\hline 6.49 & $91 \mathrm{PA}$ & KBi & 2 & 0.114 & 1 & & 7.06 & 88 & RA & KB I & 2 & 0.124 & 1 \\
\hline 6.52 & $7 \mathbf{L U}$ & KAI & 1 & 0.229 & $1,2,3$ & & 7.06 & 64 & $G D$ & KB2 & 1 & 0.248 & $1,2,3$ \\
\hline 6.55 & $67 \mathrm{HO}$ & KBI & 1 & 3.230 & 1 & & 7.06 & 87 & $F R$ & KB2 & 2 & 0.124 & 1 \\
\hline 0.60 & $3 \cap \mathrm{TH}$ & KB5 & 2 & 0.116 & 1,2 & & 7.09 & 69 & $T M$ & KA2 & 1 & 0.249 & $1,2.3$ \\
\hline 6.60 & $97 \mathrm{BK}$ & KA2 & 2 & 0.116 & 1 & $t$ & 7.12 & 94 & PU & KA2 & 2 & 0.125 & 1 \\
\hline 6.67 & 66 DY & KB2 & 1 & 0.232 & $1,2,3$ & & 7.17 & 68 & $E R$ & KAI & 1 & 0.252 & $1,2,3$ \\
\hline
\end{tabular}




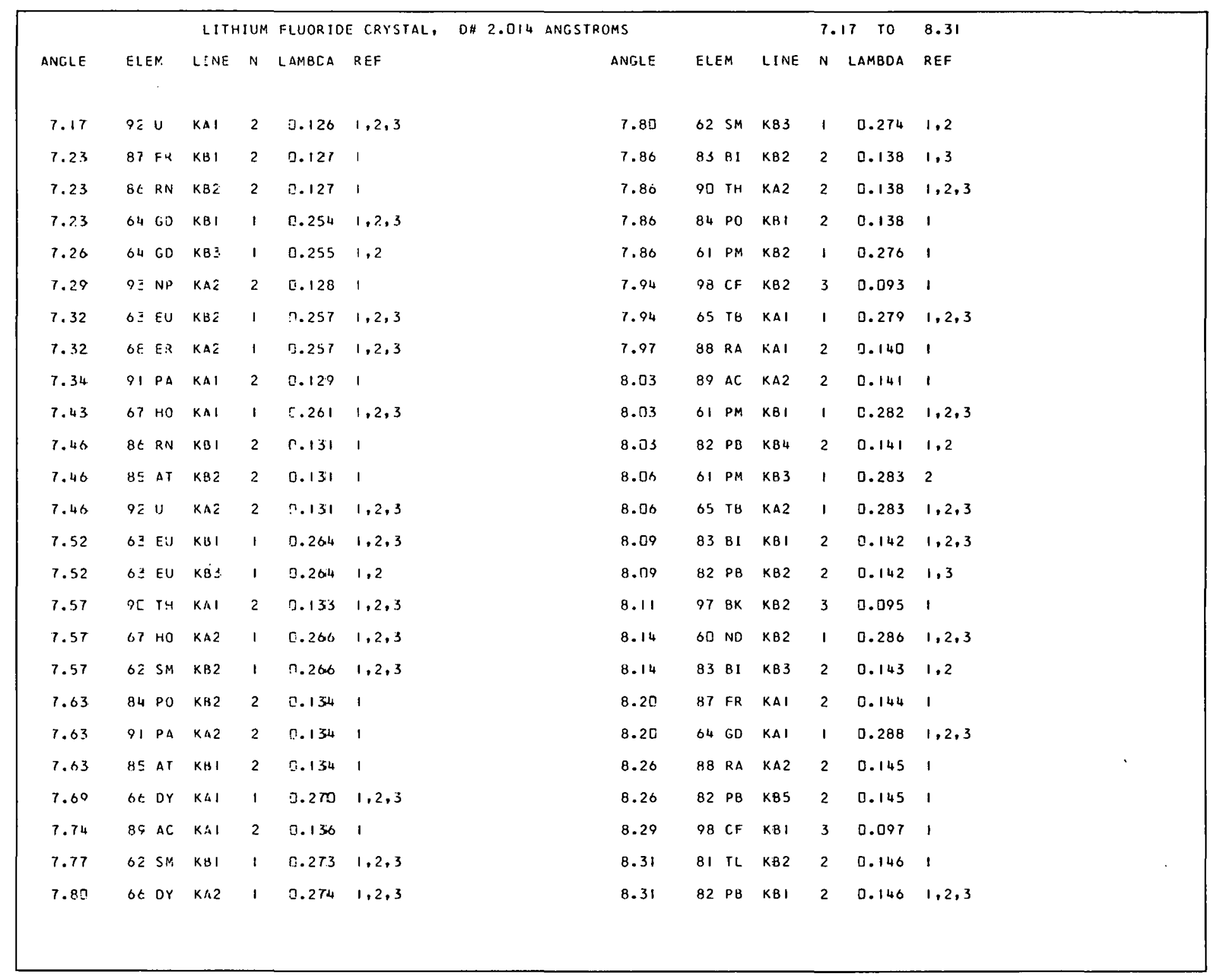




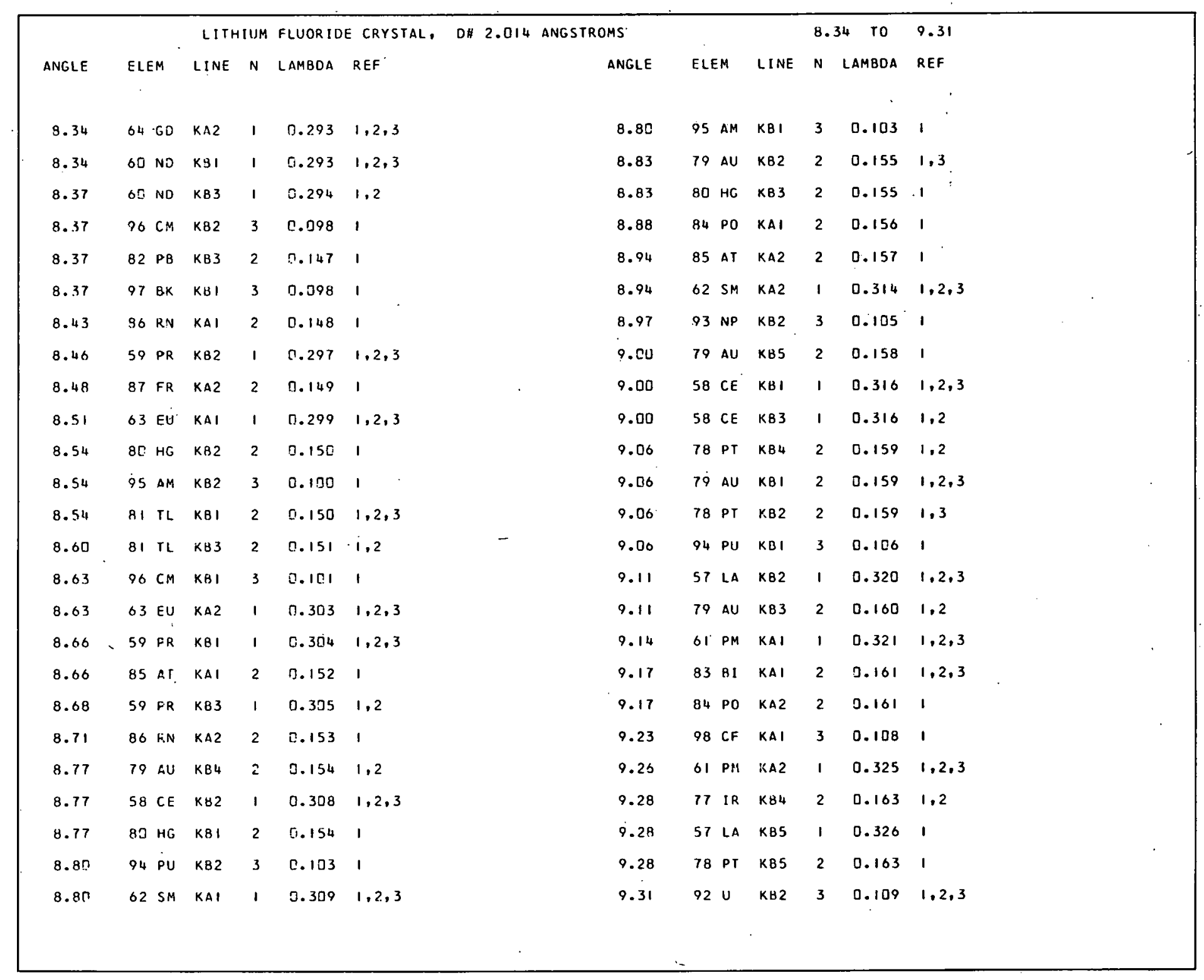




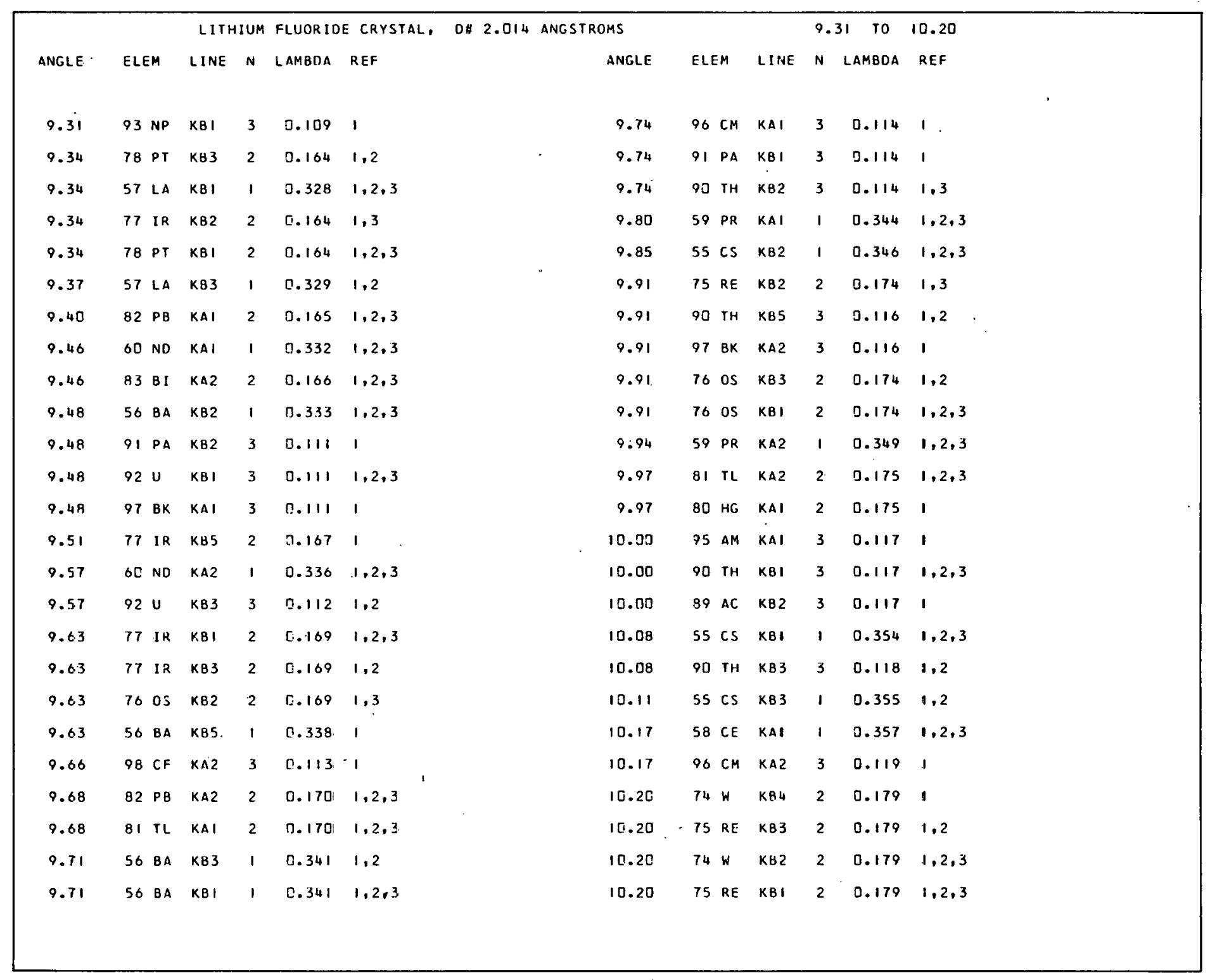




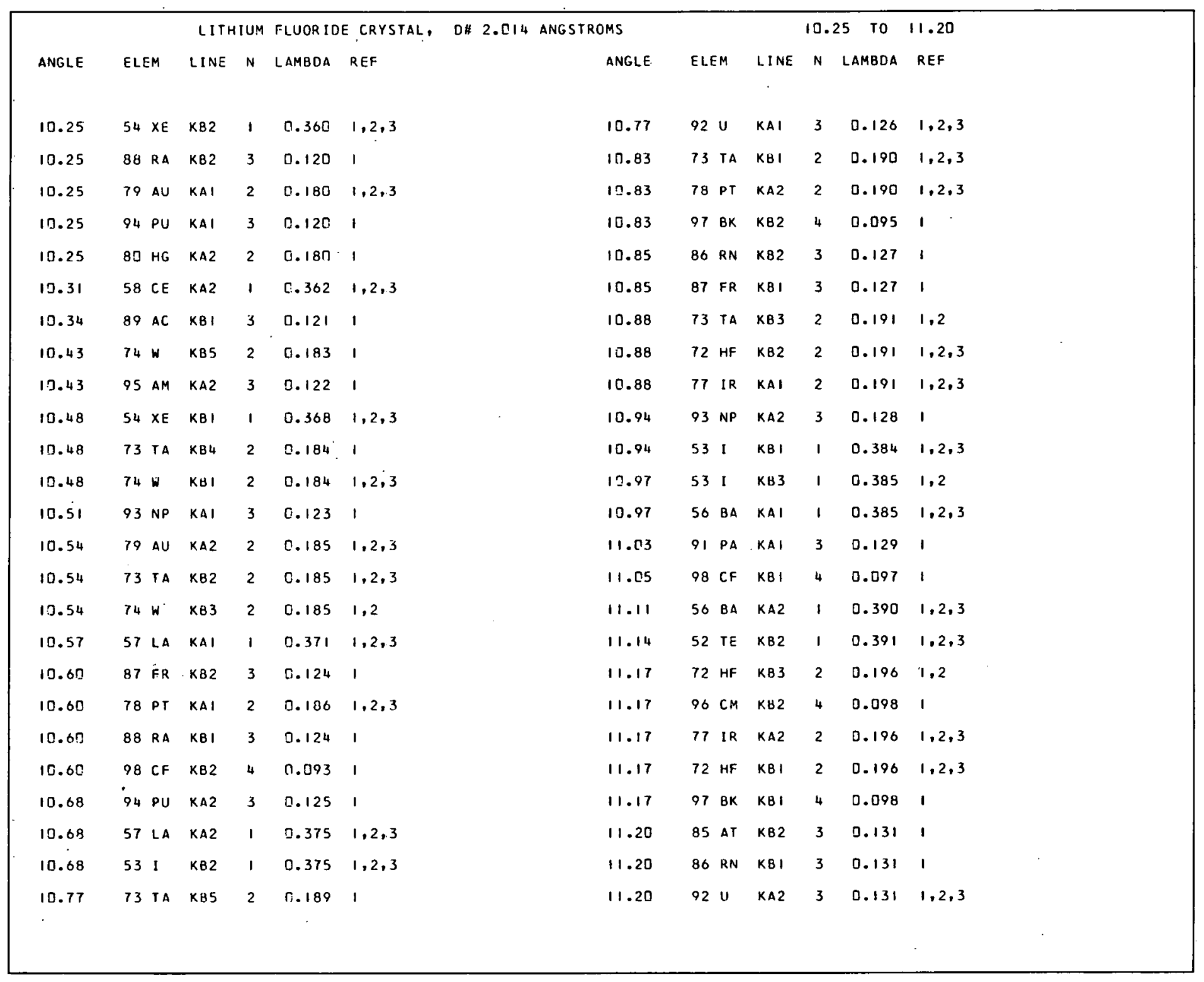




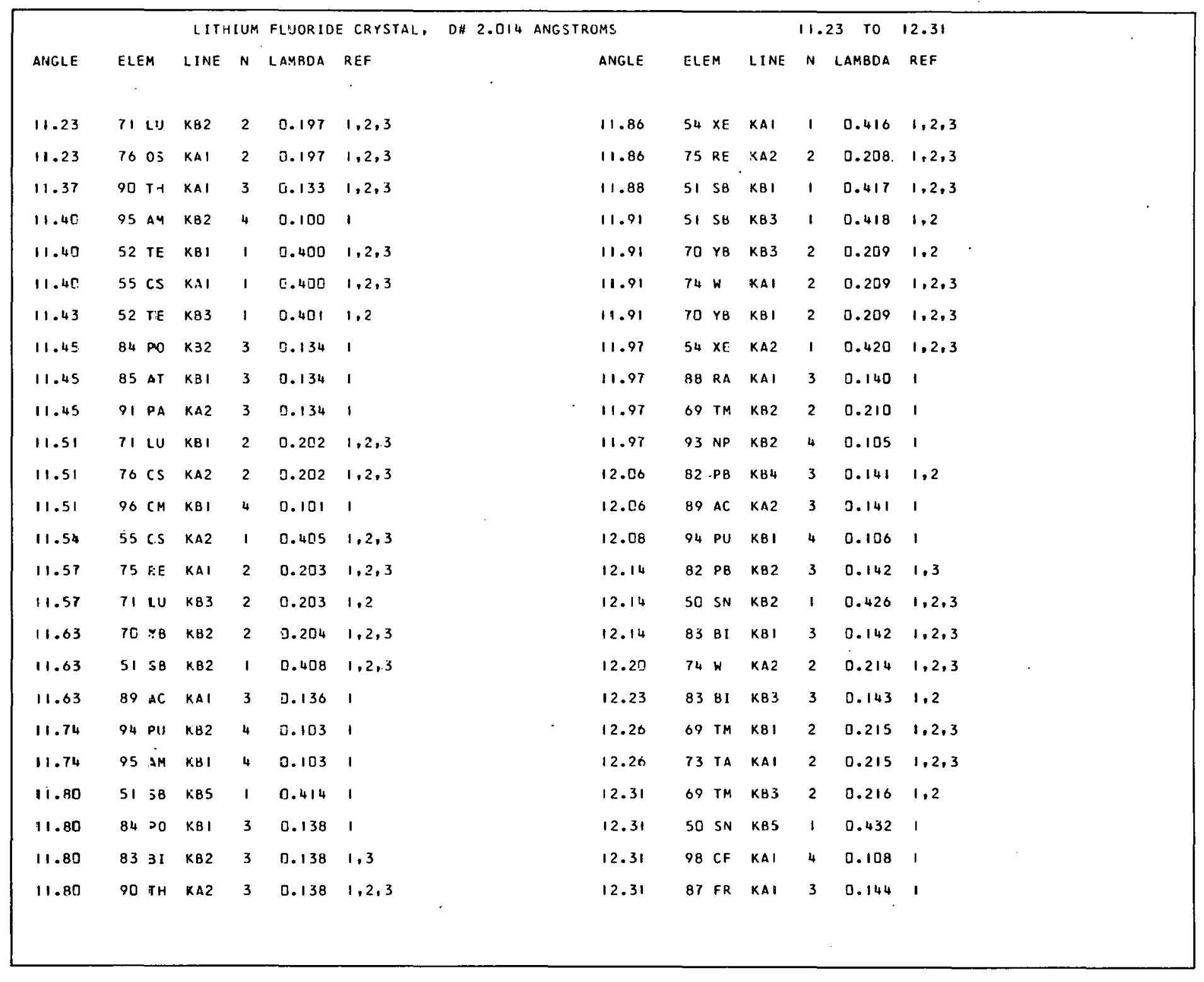




\begin{tabular}{|c|c|c|c|c|c|c|c|c|c|c|c|c|c|c|}
\hline & & LITH & IUM & FLUOR 10 & E CRYSTAL, & $0 \# 2.014$ & ANGSTROMS & & & & 12. & 34 TO & 13.26 & \\
\hline ANGLE & ELEM & LINE & $\mathbf{N}$ & LAMBDA & REF & & ANGLE & ELE & $E M$ & LINE & $N$ & LAMBDA & REF & \\
\hline & & & & & & & & & & & & & 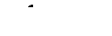 & \\
\hline 12.34 & $53 \mathrm{I}$ & KAI & 1 & 0.433 & $1,2,3$ & & 12.83 & 81 & $\mathrm{TL}$ & K8I & 3 & 0.150 & $1,2,3$ & \\
\hline 12.37 & $68 E R$ & K82 & 2 & 0.217 & $1,2,3$ & & 12.86 & 49 & IN & KBS & 1 & 0.451 & 1 & \\
\hline 12.40 & $88 R A$ & KA2 & 3 & 0.145 & 1 & . & 12.86 & 52 & $T E$ & KAI & 1 & 0.451 & $1,2,3$ & \\
\hline 12.40 & $50 \mathrm{SN}$ & KBI & 1 & 0.435 & $1,2,3$ & & 12.89 & 98 & $\mathrm{CF}$ & KA2 & 4 & 0.113 & 1 & \\
\hline 12.40 & $82 \mathrm{~PB}$ & KB5 & 3 & 0.145 & 1 & & 12.91 & 81 & $\mathrm{TL}$ & KB 3 & 3 & 0.151 & 1,2 & \\
\hline 12.43 & $92 \mathrm{U}$ & KB2 & 4 & 0.109 & $1,2,3$ & & 12.94 & 72 & $\mathrm{HF}$ & KA2 & 2 & 0.227 & $1,2,3$ & \\
\hline 12.43 & $50 \mathrm{SN}$ & KB3 & 1 & 0.436 & 1,2 & & 12.97 & 49 & IN & K83 & 1 & 0.455 & 1,2 & \\
\hline 12.43 & $93 \mathrm{NP}$ & KBI & 4 & 0.109 & 1 & & 12.97 & 4.9 & IN & KBI & 1 & 0.455 & $1,2,3$ & \\
\hline 12.48 & $81 \mathrm{TL}$ & $K B 2$ & 3 & 0.146 & 1 & & 13.00 & 91 & $P A$ & KB I & 4 & 0.114 & 1 & \\
\hline 12.48 & $53 \mathrm{I}$ & KA2 & 1 & 0.438 & $1,2,3$ & & 13.00 & 90 & $\mathrm{TH}$ & K82 & 4 & 0.114 & 1,3 & \\
\hline 12.48 & $82 P B$ & KBI & 3 & 5.146 & $1,2,3$ & & 13.00 & 52 & $T E$ & KA2 & 1 & 0.456 & $1,2,3$ & . \\
\hline 12.54 & $73 \mathrm{TA}$ & KA2 & 2 & $0.22 \mathrm{D}$ & $1,2,3$ & & 13.00 & 85 & AT & KAI & 3 & 0.152 & 1 & \\
\hline 12.57 & $82 \mathrm{~PB}$ & KB3 & 3 & 0.147 & 1. & & 13.00 & 96 & $C M$ & KAI & 4 & 0.114 & 1 & \\
\hline 12.66 & $97 \mathrm{BK}$ & KAI & 4 & 0.111 & 1 & & 13.06 & 71 & LU & XAI & 2 & 0.229 & $1,2,3$ & \\
\hline 12.66 & $86 \mathrm{RN}$ & KAI & 3 & 0.148 & 1 & & 13.09 & 86 & RN & KA2 & 3 & 0.153 & 1 & \\
\hline 12.66 & $91 \mathrm{PA}$ & KB2 & 4 & 0.111 & 1 & · & 13.11 & 67 & HO & KBI & 2 & 0.230 & 1 & \\
\hline 12.66 & $72 \mathrm{HF}$ & KAI & 2 & 0.222 & $1,2,3$ & & 13.17 & 79 & $\Delta U$ & KB4 & 3 & 0.154 & 1,2 & \\
\hline 12.66 & $92 \mathrm{U}$ & KBI & 4 & 0.111 & $1,2,3$ & & 13.17 & 80 & HG & KBI & 3 & 0.154 & 1 & \\
\hline 12.69 & 49 IN & KB2 & 1 & 0.445 & $1,2,3$ & & 13.23 & 66 & DY & KB2 & 2 & 0.232 & $1,2,3$ & \\
\hline 12.71 & $68 \mathrm{ER}$ & KB3 & 2 & 0.223 & 1,2 & & 13.23 & 97 & BK & KA2 & 4 & 0.116 & 1 & \\
\hline 12.71 & $68 \mathrm{ER}$ & KBI & 2 & 0.223 & $1,2,3$ & & 13.23 & 90 & TH & KBS & 4 & 0.116 & 1,2 & \\
\hline 12.74 & $87 \mathrm{FR}$ & KA2 & 3 & 0.149 & 1 & & 13.26 & 98 & $C F$ & KB2 & 5 & 0.093 & 1 & \\
\hline 12.77 & $92 \cup$ & $K B 3$ & 4 & 0.112 & 1,2 & & 13.26 & 80 & $H G$ & KB3 & 3 & 0.155 & 1 & \\
\hline 12.77 & $67 \mathrm{HO}$ & KB2 2 & 2 & 0.224 & 1 & & 13.26 & 79 & $A U$ & KB2 2 & 3 & 0.155 & 1,3 & \\
\hline 12.83 & $8 \mathrm{CHG}$ & KB2 & 3 & 0.150 & 1 & & 13.26 & 48 & $C D$ & $K \cdot B 2$ & 1 & 0.465 & $1,2,3$ & \\
\hline
\end{tabular}




\begin{tabular}{|c|c|c|c|c|c|c|c|c|c|c|c|c|c|c|}
\hline & & LITH & IU:1 & & E CRYSTAL, & DE $2.014 \mathrm{~A}$ & ANGSTROMS & & & & 13. & $34 T 0$ & 14.17 & \\
\hline ANGLE & ELEM & LINE & $N$ & LCMBOA & REF & & ANGLE & ELE & & I. INE & N & LAMBDA & REF & \\
\hline 13.34 & $84 P 0$ & KAI & 3 & 0.156 & 1 & & 13.77 & 83 & BI & KAI & 3 & 0.161 & $1,2,3$ & \\
\hline 13.34 & 95 AM & KAI & 4 & 0.117 & 1 & & 13.77 & 84 & PO & KA2 & 3 & 0.161 & 1 & \\
\hline 13.34 & $9[\mathrm{TH}$ & KBI & 4 & 3.117 & $1,2,3$ & & 13.80 & 89 & $A C$ & $K B I$ & 4 & 0.121 & 1 & \\
\hline 13.34 & $89 A C$ & $K B 2$ & 4 & 0.117 & 1 & & 13.83 & 98 & CF & KBI & 5 & 0.097 & 1 & \\
\hline 13.34 & $71 \mathrm{LU}$ & KA2 & 2 & 0.234 & $1,2,3$ & & 13.89 & 47 & $A G$ & KB2 & 1 & 0.487 & $1,2,3$ & \\
\hline 13.45 & $51 \mathrm{SB}$ & KAI & 1 & 3.475 & $1,2,3$ & & 13.92 & 69 & TM & KAI & 2 & 0.244 & $1,2,3$ & \\
\hline 13.43 & $85 \mathrm{AT}$ & KA2 & 3 & $0: 157$ & 1 & & 13.92 & 95 & $A M$ & KA2 & 4 & 0.122 & 1 & \\
\hline 13.46 & $90 \mathrm{TH}$ & KB3 & 4 & 0.118 & 1,2 & & 13.95 & 78 & PT & KBS & 3 & 0.163 & 1 & \\
\hline 13.52 & of or & KBI & 2 & 0.237 & $1,2,3$ & & 13.95 & 77 & I R & $K .84$ & 3 & 0.163 & 1,2 & \\
\hline 13.52 & $79 \mathrm{AU}$ & KBS & 3 & 0.158 & 1 & & 13.97 & 96 & CM & $K 82$ & 5 & 0.098 & 1 & \\
\hline 13.52 & $70 Y 9$ & KAI & 2 & {$[.237$} & $1,2,3$ & & 13.97 & 97 & $B K$ & KBI & 5 & 0.098 & 1 & \\
\hline 13.54 & $48 \mathrm{CD}$ & KBI & 1 & 0.475 & $1,2,3$ & & 14.00 & 50 & SN & KAI & 1 & 0.491 & $1,2,3$ & \\
\hline 13.54 & 5153 & KA2 & 1 & 0.475 & $1,2,3$ & & 14.03 & 65 & $T B$ & KBI & 2 & 0.246 & $1,2,3$ & \\
\hline 13.54 & $97 \mathrm{BK}$ & $\mathrm{KB2}$ & 5 & 0.095 & 1 & & 14.03 & 65 & TH & KB 3 & 2 & 0.246 & 1,2 & \\
\hline 13.57 & 4800 & $K B 3$ & 1 & 0.476 & 1.2 & & 14.03 & 78 & PT & $K B I$ & 3 & 0.164 & $1,2,3$ & \\
\hline 13.57 & $66 \mathrm{Dr}$ & KB3 & 2 & 0.238 & 1,2 & & 14.03 & 77 & IR & KB2 & 3 & 0.164 & 1,3 & \\
\hline 13.57 & $96 \mathrm{C.4}$ & KA2 & 4 & 3.118 & 1 & & 14.03 & 93 & NP & KaI & 4 & 0.123 & 1 & \\
\hline $13.6 r_{3}$ & $78 \mathrm{PT}$ & KB2 & 3 & 0.159 & 1,3 & & 14.03 & 78 & PT & $\mathrm{KB3}$ & 3 & 0.164 & 1.2 & $\cdots$ \\
\hline $13.6 \pi$ & $78 \mathrm{PI}$ & KB 4 & 3 & 0.159 & 1,2 & & 14.06 & 47 & $A G$ & KB5 & 1 & 0.493 & 1 & \\
\hline $13: 60$ & $79 \mathrm{~A}^{9} \mathrm{~J}$ & KBI & 3 & 6.159 & $1,2,3$ & & 14.12 & 82 & PB & KAI & 3 & 0.165 & $1,2,3$ & \\
\hline 13.69 & $65 \mathrm{~TB}$ & $\mathrm{~KB} 2$ & 2 & $\Xi .240$ & $1,2.3$ & & 14.12 & 50 & SN & $K A 2$ & 1 & 0.495 & $1,2,3$ & \\
\hline 13.69 & $88 R A$ & KB 2 & 4 & 5.120 & 1 & & 14.15 & 87 & $F R$ & $\mathrm{~K} 32$ & 4 & 0.124 & 1 & \\
\hline 13.69 & $79 \mathrm{AJ}$ & $K 83$ & 3 & 0.160 & 1,2 & & 14.15 & 64 & GD & $k 32$ & 2 & 0.248 & $1,2,3$ & \\
\hline 13.65 & $94 \mathrm{PU}$ & KAI & 4 & $r .1 \leq 0$ & 1 & & 14.15 & 88 & $R A$ & K३3 I & 4 & 0.124 & 1 & \\
\hline 13.74 & $70 \mathrm{YB}$ & KAZ & 2 & 0.241 & $1,2,3$ & & 14.17 & 47 & $A G$ & $K B I$ & 1 & 0.497 & $1,2,3$ & \\
\hline
\end{tabular}




\begin{tabular}{|c|c|c|c|c|c|c|c|c|c|c|c|c|c|}
\hline \multirow[b]{2}{*}{ ANGLE } & \multirow[b]{2}{*}{ ELEM } & \multicolumn{2}{|c|}{ LITHIUM } & \multirow{2}{*}{$\begin{array}{l}\text { FLUOR IOEE } \\
\text { LAMBDA }\end{array}$} & \multirow{2}{*}{$\begin{array}{l}\text { E CRYSTAL, } \\
\text { REF }\end{array}$} & \multirow[t]{2}{*}{$D \$ 2.014$} & \multicolumn{4}{|l|}{ ANGSTROMS } & \multicolumn{3}{|c|}{14.20 TO 15.26} \\
\hline & & LINE & $N$ & & & & ANGLE & ELEI & & LINE & $N$ & LAMBDA & REF \\
\hline 14.20. & $83 \mathrm{BI}$ & KA2 & 3 & 0.166 & $1,2,3$ & & 14.69 & 95 & $A M$ & KBI & 5 & 0.103 & 1. \\
\hline $14.20^{\circ}$ & $69 \mathrm{TM}$ & KA2 & 2 & 0.249 & $1,2,3$ & & 14.72 & 911 & PA & KAI & 4 & 0.129 & 1 \\
\hline 14.20 & $47 \triangle G$ & KB3 & 1 & $\pi .498$ & 1,2 & & 14.75 & 49 & IN & KA2 & 1 & 0.517 & $1,2,3$ \\
\hline 14.26 & $94 \mathrm{PU}$ & KA2 & 4 & 0.125 & 1 & & 14.75 & 46 & PD & $K B 5$ & 1 & 0.517 & 1 \\
\hline 14.26 & 95 Ailt & KB2 & 5 & 0.100 & 1 & & 14.86 & 461 & PD & KBI & 1 & 0.521 & $1,2,3$ \\
\hline 14.29 & 7718 & K85 & 3 & 0.167 & 1 & & 14.86 & 46 & PD & KB3 & 1 & 0.521 & 1,2 \\
\hline 14.38 & $92 \mathrm{U}$ & KAI & 4 & 0.126 & $1,2,3$ & & 14.89 & 76 & os & KB 3 & 3 & 0.174 & 1,2 \\
\hline 14.38 & $68 \mathrm{ER}$ & KAI & 2 & 0.252 & $1,2,3$ & & 14.89 & 76 & os & $K B I$ & 3 & 0.174 & $1,2,3$ \\
\hline 14.40 & $96 \mathrm{~cm}$ & $K B I$ & 5 & 0.101 & 1 & & 14.89 & 75 & RE & KB2 & 3 & 0.174 & 1,3 \\
\hline 14.46 & 77 IR & KBI & 3 & 0.169 & $1,2,3$ & & 14.89 & 671 & HO & KAI & 2 & 0.261 & $1,2,3$ \\
\hline 14.46 & 7718 & KB3 & 3 & $n .169$ & 1,2 & & 14.95 & 86 & RN & KB I & 4 & 0.131 & 1 \\
\hline 14.46 & 7605 & KB2 & 3 & 0.169 & 1,3 & . & 14.95 & 85 & $A T$ & KB2 & 4 & 0.131 & 1 \\
\hline 14.49 & 6460 & KB I & 2 & 0.254 & $1,2,3$ & & 14.95 & 921 & u & KA2 & 4 & 0.131 & $1,2,3$ \\
\hline 14.49 & 86 RN & KB2 & 4 & 0.127 & 1 & & 14.98 & 801 & HG & KAI & 3 & 0.175 & 1 \\
\hline 14.49 & $87 \mathrm{FR}$ & KBI & 4 & C.127 & 1 & & 14.98 & 931 & NP & KB2 & 5 & 0.105 & 1 \\
\hline 14.55 & $81 \mathrm{IL}$ & KAI & 3 & 0.170 & $1,2,3$ & & 14.98 & 81 & $\mathrm{TL}$ & KA2 & 3 & 0.175 & $1,2,3$ \\
\hline 14.55 & 6460 & KB3 & 2 & 0.255 & 1,2 & & 15.06 & 63 & EU & KB3 & 2 & 0.264 & 1.2 \\
\hline 14.55 & $40 P 0$ & KB2 & 1 & 5.510 & $1,2,3$ & & 15.06 & 63 & EU & KB I & 2 & 0.264 & $1,2,3$ \\
\hline 14.55 & $82 \mathrm{~PB}$ & KA2 & 3 & 0.170 & $1,2,3$ & & 15.12 & 94 & PU & KEI & 5 & 0.106 & 1 \\
\hline 14.60 & $93 \mathrm{NP}$ & KA2 & 4 & 0.128 & 1 & & 15.12 & 97 & BK & LYI & 1 & 0.530 & 1 \\
\hline $14.6 ?$ & 49 IN & KAI & 1 & 0.512 & $1,2,3$ & & 15.18 & 62 & SM & KB2 & 2 & 0.266 & $1,2,3$ \\
\hline 14.66 & $63 \mathrm{EU}$ & KB2 & 2 & c. 257 & $1,2,3$ & & 15.18 & 671 & HO & KA2 & 2 & 0.266 & $1,2,3$ \\
\hline 14.66 & $68 E R$ & KA2 & 2 & $\because .257$ & $1,2,3$ & & 15.18 & 90 & TH & KAI & 4 & 0.133 & $1,2,3$ \\
\hline 14.69 & $98 \mathrm{CF}$ & LYI & 1 & 0.515 & 1 & & 15.26 & 45 & RH & $K B 2$ & 1 & 0.535 & $1,2,3$ \\
\hline 14.69 & $94 \mathrm{PU}$ & KB2 & 5 & {$[\because .103$} & 1 & & 15.26 & 48 & CO & KAI & 1 & 0.535 & $1,2,3$ \\
\hline
\end{tabular}




\begin{tabular}{|c|c|c|c|c|c|c|c|c|c|c|c|c|c|}
\hline \multirow[b]{2}{*}{ ANGLE } & \multicolumn{3}{|c|}{ LITHIUM } & \multirow{2}{*}{\multicolumn{2}{|c|}{$\begin{array}{l}\text { FLUORIDE CRYSTAL, } \\
\text { LAMBDA REF }\end{array}$}} & \multirow{2}{*}{ D* 2.014} & \multirow{2}{*}{$\begin{array}{l}\text { ANGSTROMS } \\
\text { ANGLE }\end{array}$} & \multicolumn{5}{|c|}{15.29 TO } & \multirow{2}{*}{$\begin{array}{l}16.18 \\
\text { REF }\end{array}$} \\
\hline & ELEM & LINE & $N$ & & & & & ELE & & LINE & $N$ & LAḾBDA & \\
\hline 15.29 & $84 P 0$ & KB2 & 4 & 0.134 & 1 & & 15.75 & 01 & PM & KB2 & 2 & 0.276 & 1 \\
\hline 15.29 & $85 \mathrm{AT}$ & $K B 1$ & 4 & 0.134 & 1 & & 15.75 & 84 & po & KBI. & 4 & 0.138 & 1 \\
\hline 15.29 & $91 P 9$ & KA2 & 4 & c. 134 & 1 & & 15.75 & 83 & 81 & KB2 & 4 & 0.138 & 1,3 \\
\hline 15.32 & $75 R E$ & KBI & 3 & 0.179 & $1,2,3$ & & 15.75 & 73 & TA & $K B 4$ & 3 & 0.184 & 1 \\
\hline 15.32 & $7: 4 \quad H$ & $K 82$ & 3 & 0.179 & $1,2,3$ & & 15.84 & 74 & $w$ & $K B 3$ & 3 & 0.185 & 1,2 \\
\hline 15.32 & $75 R \equiv$ & $\mathrm{KB} 3$ & 3 & 0.179 & 1.2 & & 15.84 & 91 & PA & $K B 2$ & 5 & 0.111 & 1 \\
\hline 15.32 & $74 \mathrm{~W}$ & $K B 4$ & 3 & 0.179 & 1 & & 15.84 & 97 & BK & KAI & 5 & 0.111 & 1 \\
\hline 15.38 & $48 \mathrm{CO}$ & $K 42$ & 1 & {$[. .5 \leq 9$} & $1,2,3$ & & 15.84 & 73 & TA & $k 32$ & 3 & 0.185 & $1,2,3$ \\
\hline 15.41 & $66 \mathrm{DY}$ & KAI & 2 & 0.270 & $1,2,3$ & & 15.84 & 92 & $u$ & $k 31$ & 5 & 0.111 & $1,2,3$ \\
\hline 15.41 & 794.5 & KAI & 3 & ㅂ. $18 \mathrm{E}$ & $1,2,3$ & & 15.84 & 79 & $A U$ & KA2 & 3 & 0.185 & $1,2,3$ \\
\hline 15.41 & $8 D H G$ & $k \wedge 2$ & 3 & 0.180 & 1 & & 15.93 & 78 & $P T$ & KAI & 3 & 0.186 & $1,2,3$ \\
\hline 15.41 & $98 \mathrm{CF}$ & KAI & 5 & $0.1[8$ & 1 & & 15.93 & 65 & TB & KAI & 2 & 0.279 & $1,2,3$ \\
\hline 15.44 & $45 \mathrm{RH}$ & KBS & 1 & 0.541 & 1 & & 15.95 & 47 & $\Delta G$ & KAI & 1 & 0.559 & $1,2,3$ \\
\hline 15.52 & $89 \mathrm{AC}$ & KAI & 4 & 0.126 & 1 & & 15.98 & 92 & u & KB 3 & 5 & 0.112 & 1.2 \\
\hline 15.55 & $92 \mathrm{U}$ & $K B 2$ & 5 & $0.1[9$ & 1.2 .3 & & 15.98 & 88 & $R A$ & KAI & 4 & 0.140 & 1 \\
\hline 15.55 & $93 \mathrm{NP}$ & KBI & 5 & 0.15 .9 & 1 & & 16.04 & 95 & $A M$ & LYI & 1 & 0.562 & 1,3 \\
\hline $15.5 F$ & $45 \mathrm{RH}$ & KB3 & 1 & 0.510 & 1.2 & & 16.04 & 44 & RU & KB2 & 1 & 0.562 & $1,2,3$ \\
\hline $15.5 E$ & $45 \mathrm{RH}$ & KBI & 1 & 7.51 .6 & $1,2,3$ & & 16.10 & 61 & PM & $K B I$ & 2 & 0.282 & $1,2,3$ \\
\hline $15.5 E$ & $96 \mathrm{CM}$ & $|Y|$ & 1 & $0.5^{2} 6$ & 1 & & 10.10 & 47 & $A G$ & KA2 & 1 & 0.564 & $1,2,3$ \\
\hline $15.5 E$ & $62 \mathrm{SM}$ & KBI & 2 & 0.273 & $1,2,3$ & & 16.10 & 82 & PB & KB 4. & 4 & 0.141 & 1,2 \\
\hline $15.6 \mathrm{t}$ & $62 \mathrm{SM}$ & Kв 3 & 2 & 0.274 & 1,2 & & 16.10 & 89 & $A C$ & KA2 & 4 & 0.141 & 1 \\
\hline $15.6 \mathrm{~L}$ & to DY & $K A Z$ & 2 & 0.274 & $1,2,3$ & & 16.13 & 98 & CF & KA2 & 5 & 0.113 & 1 \\
\hline 15.67 & $74 \mathrm{~W}$ & KBS & 3 & 0.183 & 1 & & 16.15 & 65 & $T B$ & KA2 & 2 & 0.283 & $1,2,3$ \\
\hline 15.75 & $74 \mathrm{~W}$ & $K H I$ & 3 & 0.184 & $1,2,3$ & & 16.15 & 61 & PM & KB3 & 2 & 0.283 & 2 \\
\hline 15.75 & $90 \mathrm{TH}$ & $K A 2$ & 4 & 0.138 & $1,2,3$ & & 16.18 & 73 & TA & KBS & 3 & 0.189 & 1 \\
\hline
\end{tabular}




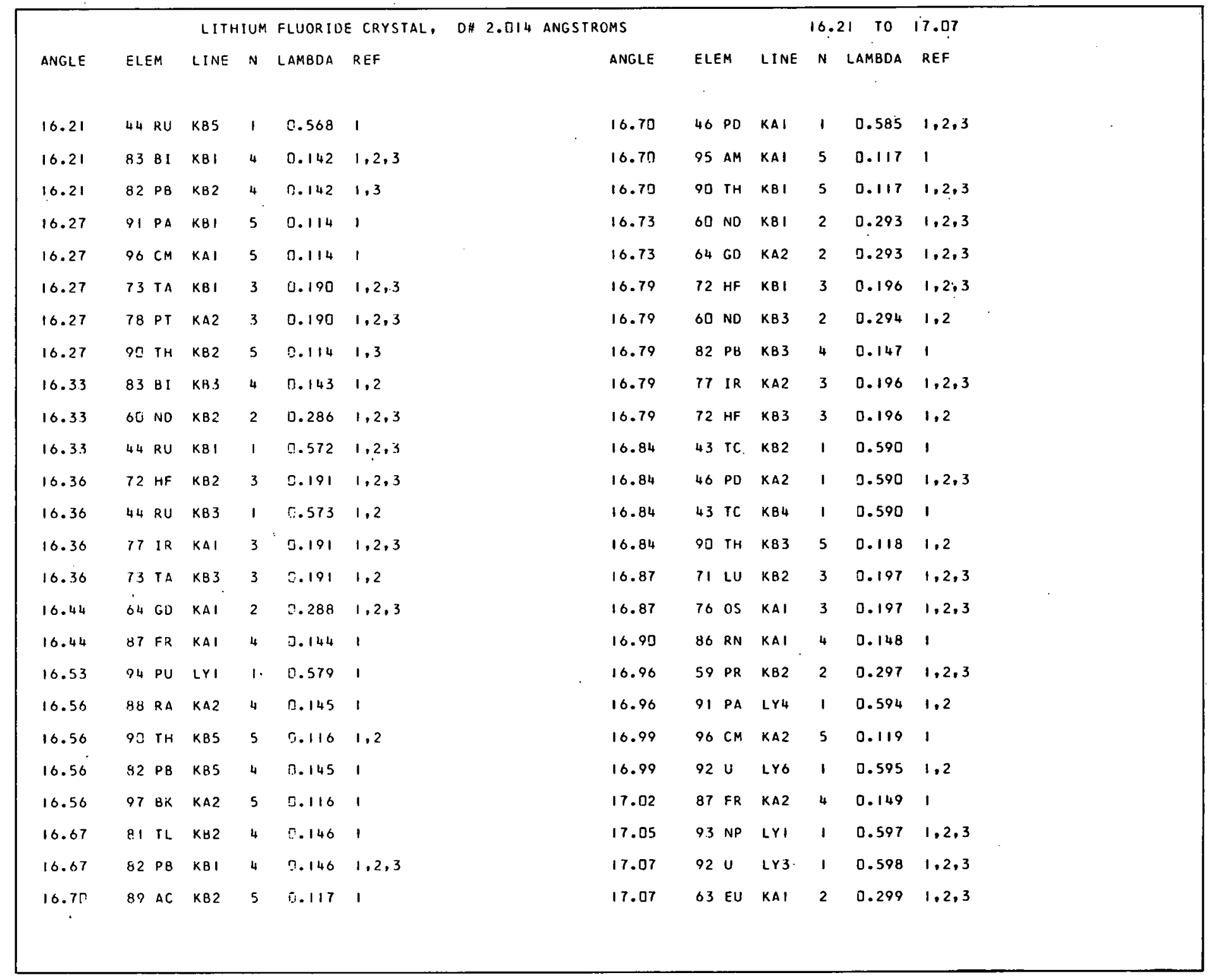




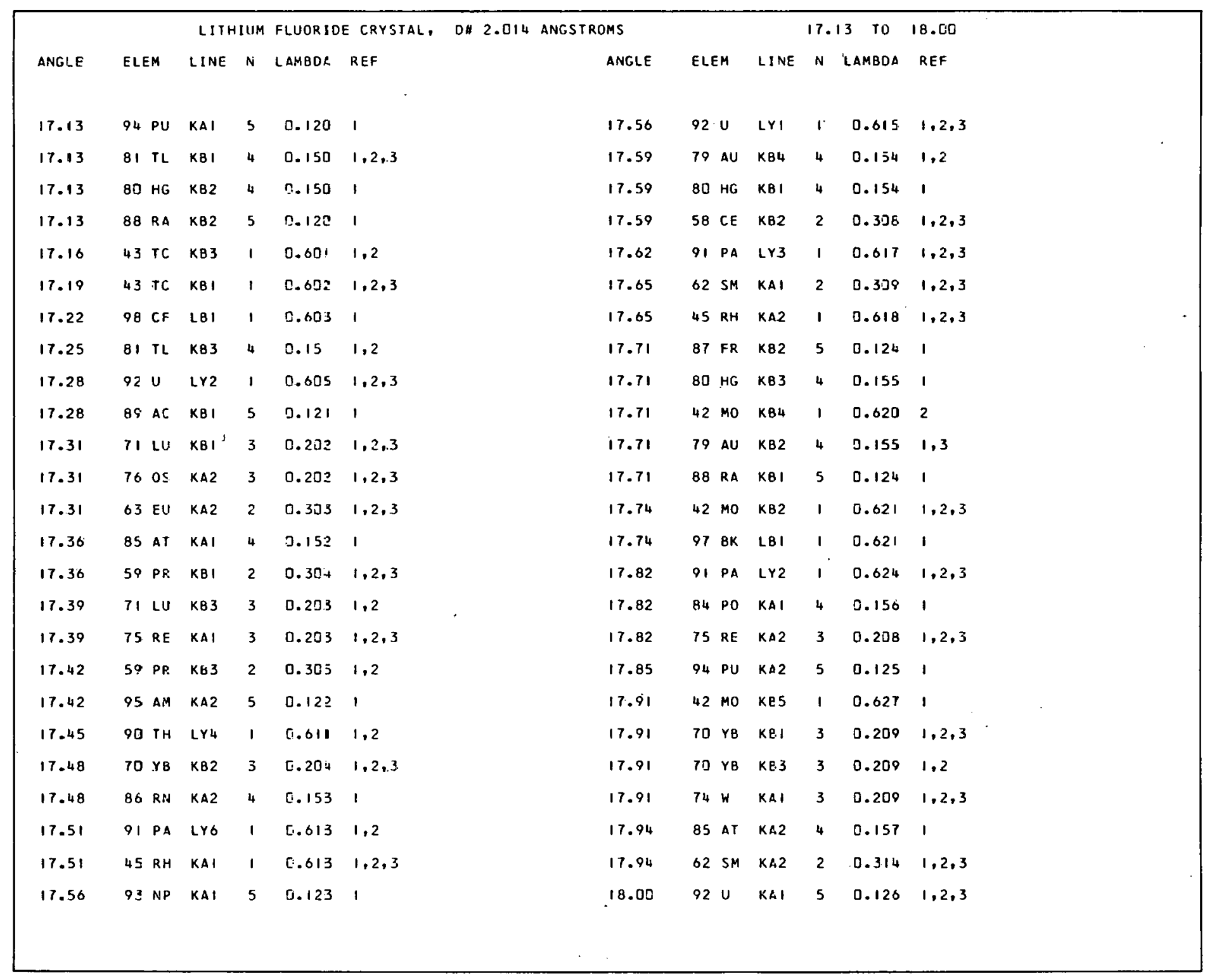




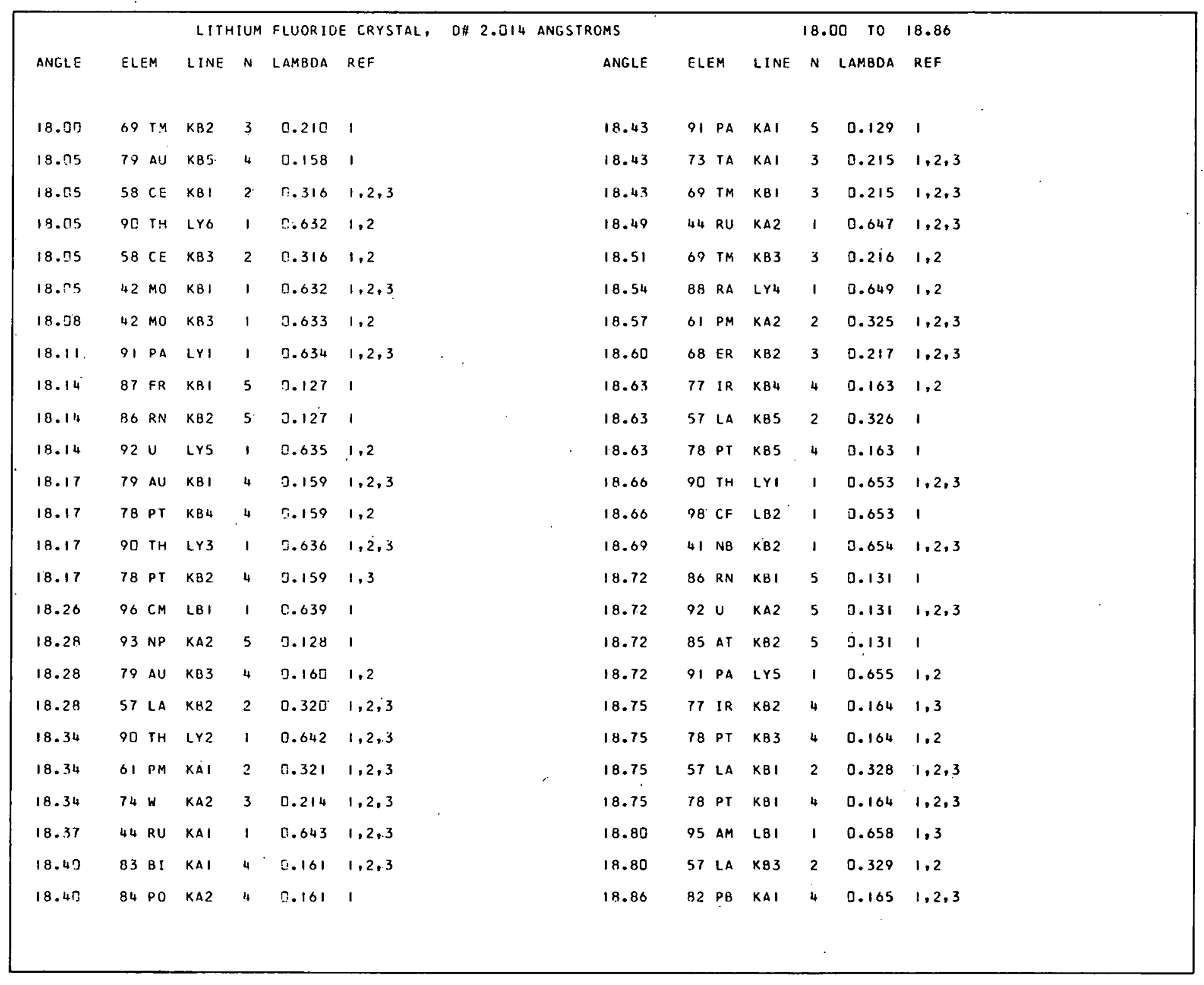




\begin{tabular}{|c|c|c|c|c|c|c|c|c|c|c|c|c|}
\hline & & LITH & IUM & FLUOR IDE & E CRYSTAL, & D*2.014 & AIIGSTROMS & & & 18. & TO & 19.90 \\
\hline ANGLE & ELEM & LINE & $N$ & LAMBDA & REF & & ANGLE & ELEM & LIVE & $N$ & LAMBDA & REF \\
\hline 18.86 & $73 \mathrm{TA}$ & KA2 & 3 & 0.220 & $1,2,3$ & & 19.32 & 77 IR & KBI & .4 & 0.189 & $1,2,3$ \\
\hline 18.36 & $41 \mathrm{NB}$ & KB5 & 1 & 0.660 & 1 & & 19.32 & $43 \mathrm{TC}$ & KA2 & 1 & 0.676 & 2,3 \\
\hline 18.78 & $60 \mathrm{ND}$ & KAI & 2 & 0.332 & $1,2,3$ & & 19.32 & $56 \mathrm{BA}$ & KBS & 2 & 0.338 & 1 \\
\hline 18.78 & $83 \mathrm{BI}$ & $K A 2$ & 4 & 0.166 & $1,2,3$ & & 19.38 & $94 \mathrm{PU}$ & LBI & 1 & $0.67 \varepsilon$ & 1 \\
\hline 19.30 & $9[T H$ & KAI & 5 & 0.133 & $1,2,3$ & & 19.44 & $89 \mathrm{AC}$ & KAI & 5 & 0.136 & 1 \\
\hline 19.13 & $72 \mathrm{HF}$ & KAI & 3 & 0.222 & $1,2,3$ & & 19.44 & $81 \mathrm{TL}$ & KAI & 4 & 0.170 & $1,2,3$ \\
\hline 19.03 & $56 \mathrm{BA}$ & $K B 2$ & 2 & 0.333 & $1,2,3$ & & 19.44 & $82 \mathrm{~PB}$ & KA2 & 4 & 0.170 & $1,2,3$ \\
\hline 19.03 & $41 \mathrm{NB}$ & KBI & 1 & C.660 & $1,2,3$ & & 19.47 & $92 \mathrm{U}$ & LB9 & 1 & 0.681. & 1.2 \\
\hline 19.03 & $41 \mathrm{NB}$ & $K B 3$ & 1 & 0.660 & 1,2 & & 19.47 & $72 \mathrm{HF}$ & $K A 2$ & 3 & 0.227 & $1,2,3$ \\
\hline 19.09 & 77 If & KBS & 4 & 0.167 & 1 & & 19.50 & $568 A$ & KB3 & 2 & 0.341 & 1,2 \\
\hline 19.12 & $68 \mathrm{ER}$ & $K \in 3$ & 3 & 0.223 & 1,2 & & 19.50 & $8 B R A$ & LY2 & 1 & 0.682 & $1,2,3$ \\
\hline 19.12 & $97 \mathrm{BK}$ & LB2 & 1 & 0.667 & 1 & & 19.50 & $56 B A$ & Ke.I & 2 & 0.341 & $1,2,3$ \\
\hline 19.12 & $68 \mathrm{ER}$ & KBI & 3 & 0.223 & $1,2,3$ & & 19.58 & $96 \mathrm{~cm}$ & $L 82$ & 1 & 0.685 & 1 \\
\hline 19.15 & $91 \mathrm{PA}$ & KA2 & 5 & {$[.134$} & 1 & & 19.64 & $71 \mathrm{LU}$ & $K A I$ & 3 & 0.229 & $1,2,3$ \\
\hline 19.15 & $85 \mathrm{AT}$ & KBI & 5 & 0.134 & 1 & & 19.64 & $92 \mathrm{U}$ & L6ID & 1 & 0.687 & 1,2 \\
\hline 19.15 & $84 P O$ & KB2 & 5 & 0.134 & 1 & & 19.67 & $59 \mathrm{PR}$ & KAI & 2 & 0.344 & $1,2,3$ \\
\hline 19.21 & $60 \mathrm{ND}$ & KA2 & 2 & 0.3 .56 & $1,2,3$ & & 19.73 & $40 \mathrm{ZR}$ & KB2 & 1 & 0.690 & $1,2,3$ \\
\hline 19.21 & $67 \mathrm{HO}$ & KB2 & 3 & 0.224 & 1 & & 19.73 & $67 \mathrm{HO}$ & KBI & 3 & 0.230 & 1 \\
\hline 19.24 & $88 \mathrm{RA}$ & LYo & 1 & 0.673 & 1,2 & & 19.73 & $90 \mathrm{TH}$ & $K A 2$ & 5 & 0.138 & $1,2,3$ \\
\hline 19.24 & $43 \mathrm{TC}$ & KAI & 1 & 0.673 & 2,3 & & 19.73 & $84 \mathrm{PO}$ & KBI & 5 & 0.138 & 1 \\
\hline 19.26 & $89 \mathrm{AL}$ & LYI & 1 & 0.67 .4 & 1 & & 19.73 & 8381 & $K B 2$ & 5 & 0.138 & 1,3 \\
\hline 19.29 & $90 \mathrm{TH}$ & LY5 & 1 & 0.675 & 1,2 & & 19.78 & $55 \mathrm{cs}$ & $K B 2$ & 2 & 0.346 & $1,2,3$ \\
\hline 19.29 & $88 R A$ & LY3 & 1 & 0.675 & $1,2,3$ & & 19.87 & 88 RA & $|Y|$ & 1 & 0.695 & $1,2,3$ \\
\hline 19.32 & 76 os & $\mathrm{K} 82$ & 4 & $0.1 \in 9$ & 1,3 & & 19.90 & $402 R$ & KBS & 1 & 0.696 & 1 \\
\hline 19.32 & $77 \mathrm{IR}$ & $K B 3$ & 4 & $0.1 \in 9$ & 1,2 & & 19.90 & 76 OS & $K 83$ & 4 & 0.174 & 1,2 \\
\hline
\end{tabular}




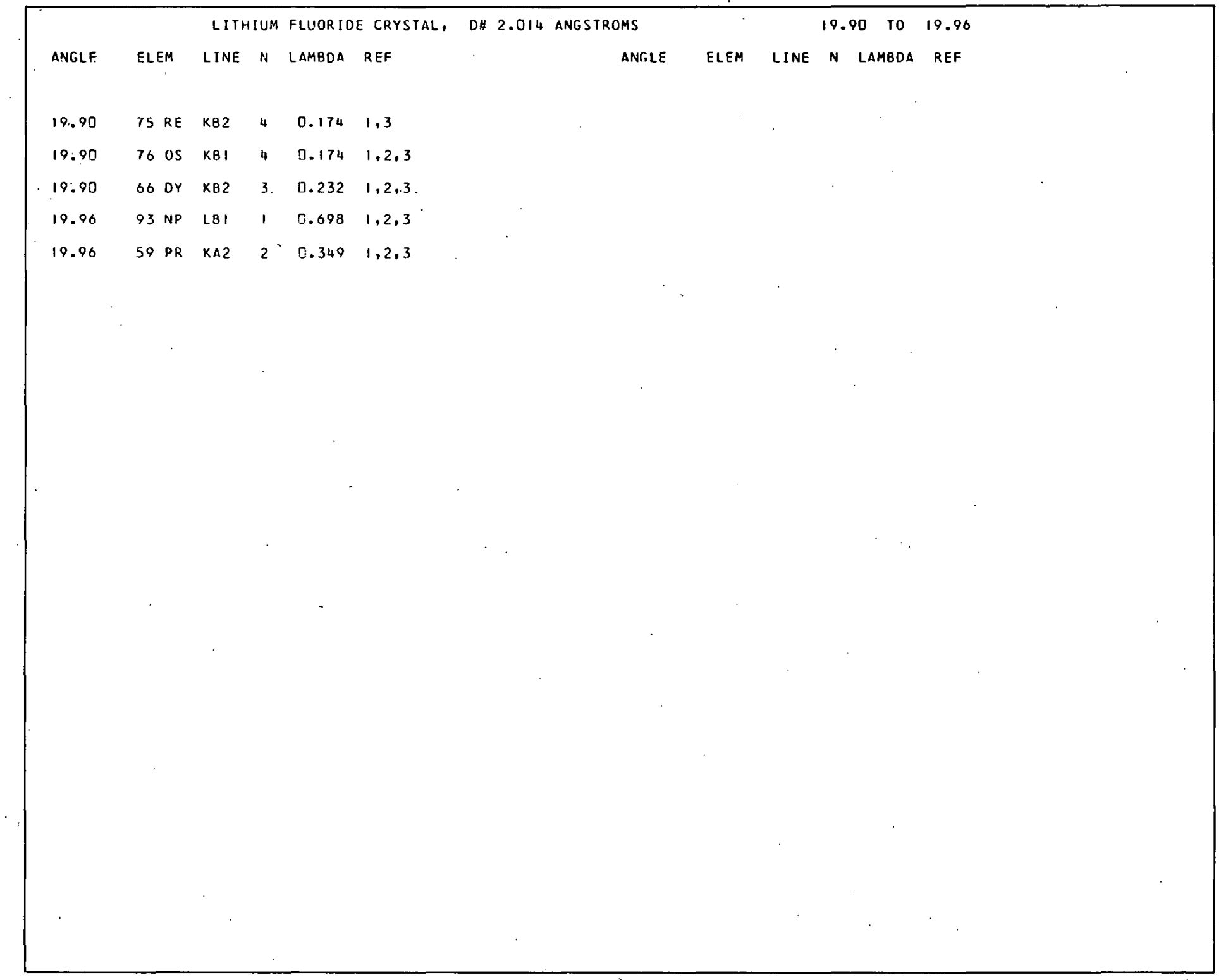




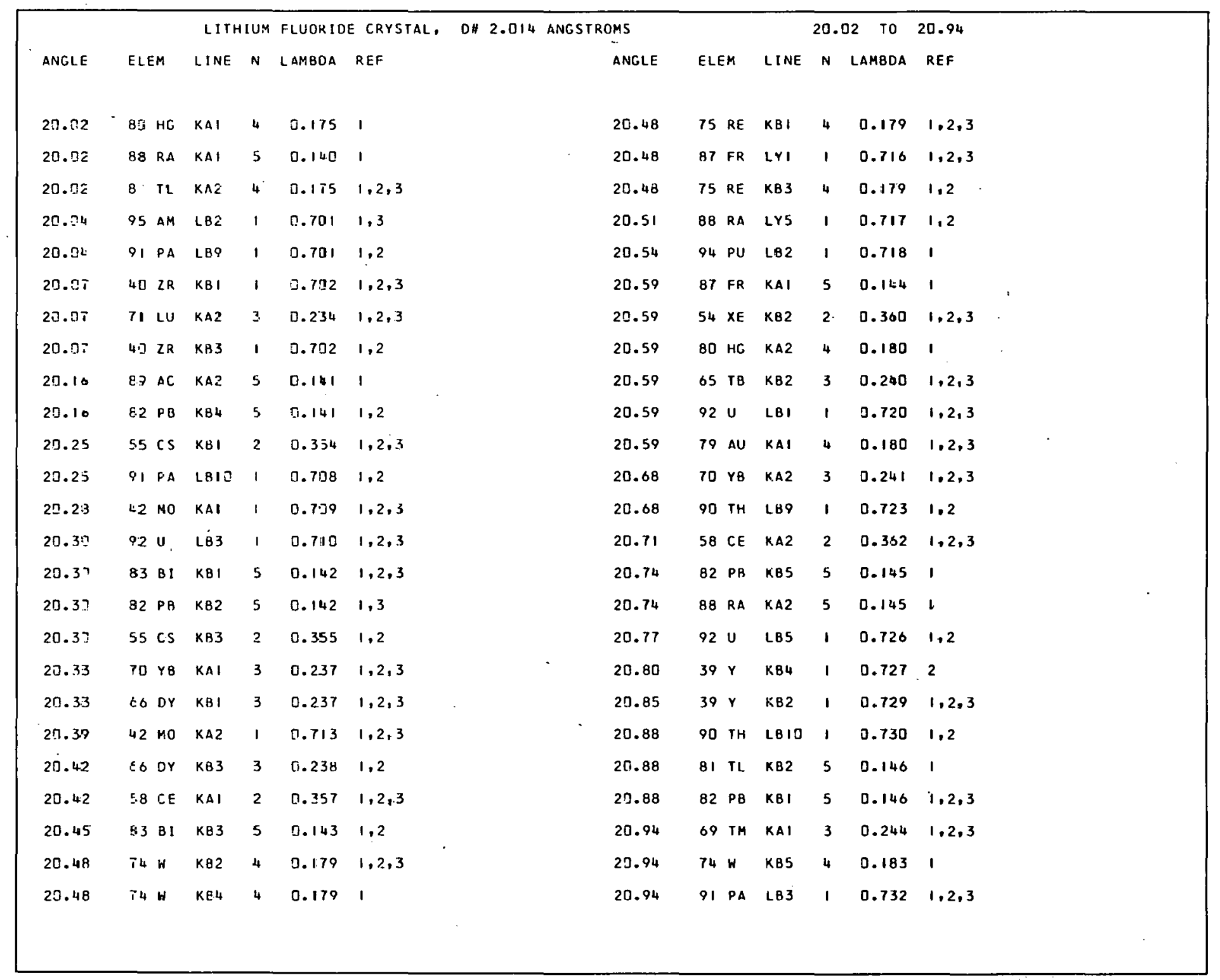




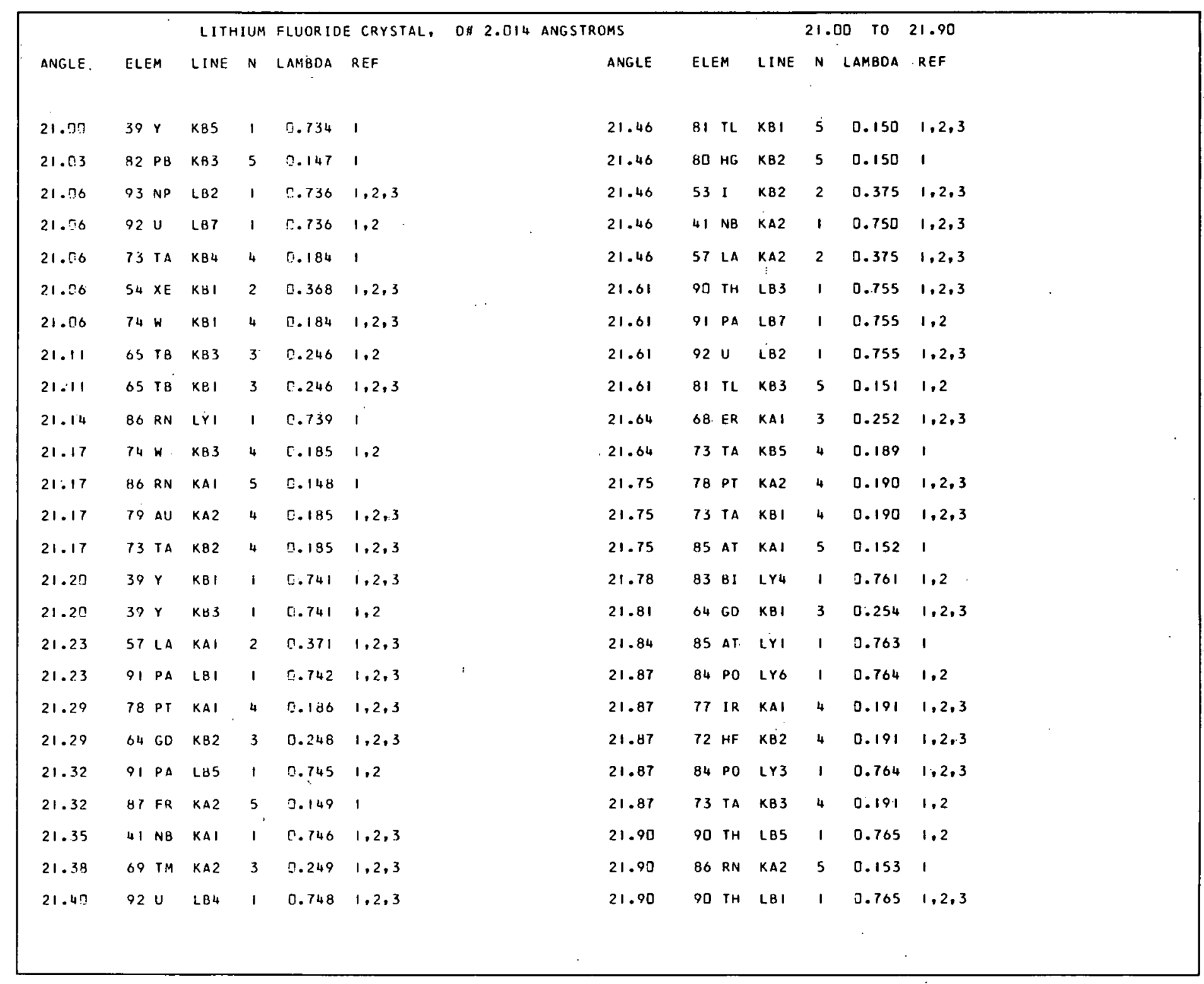




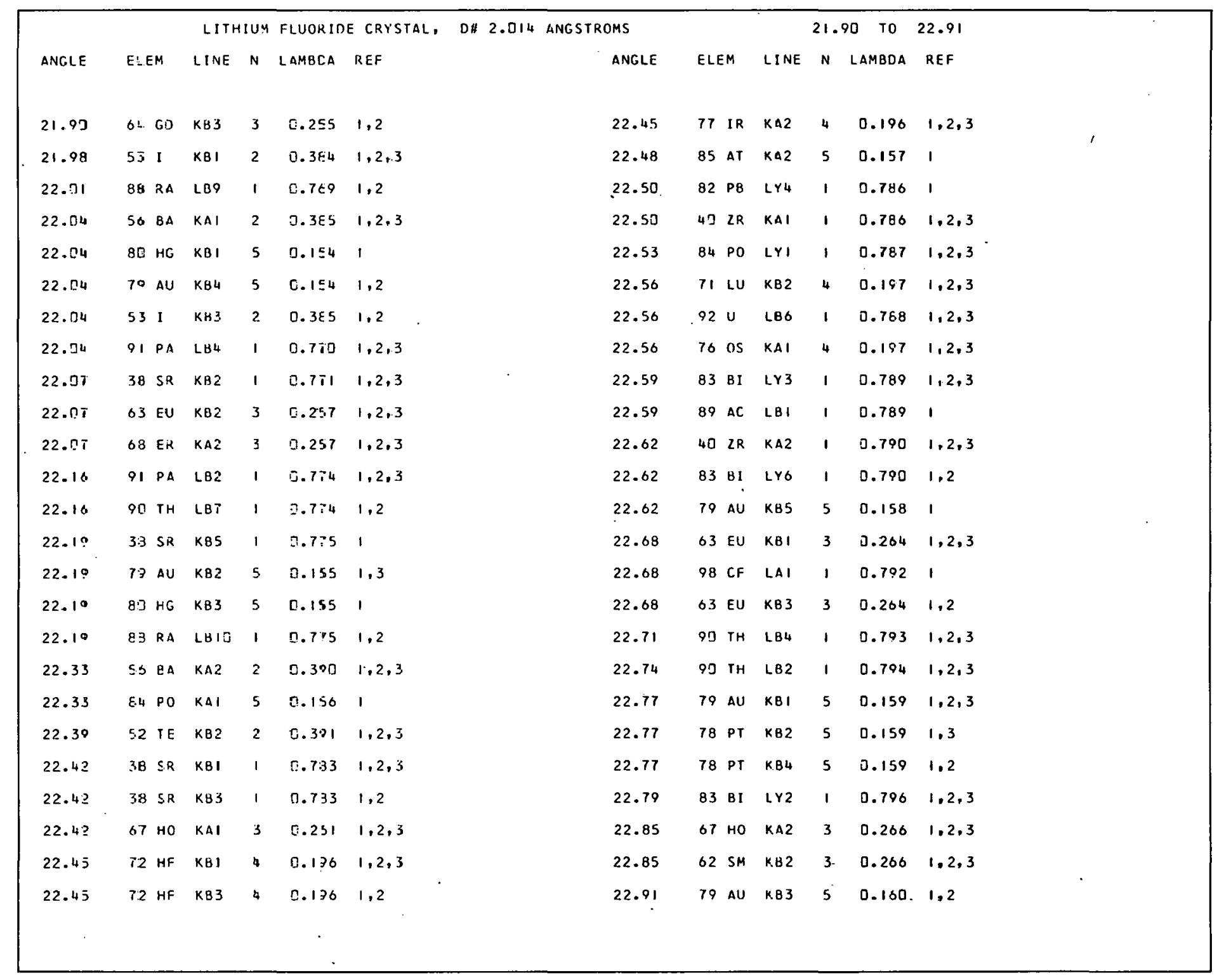




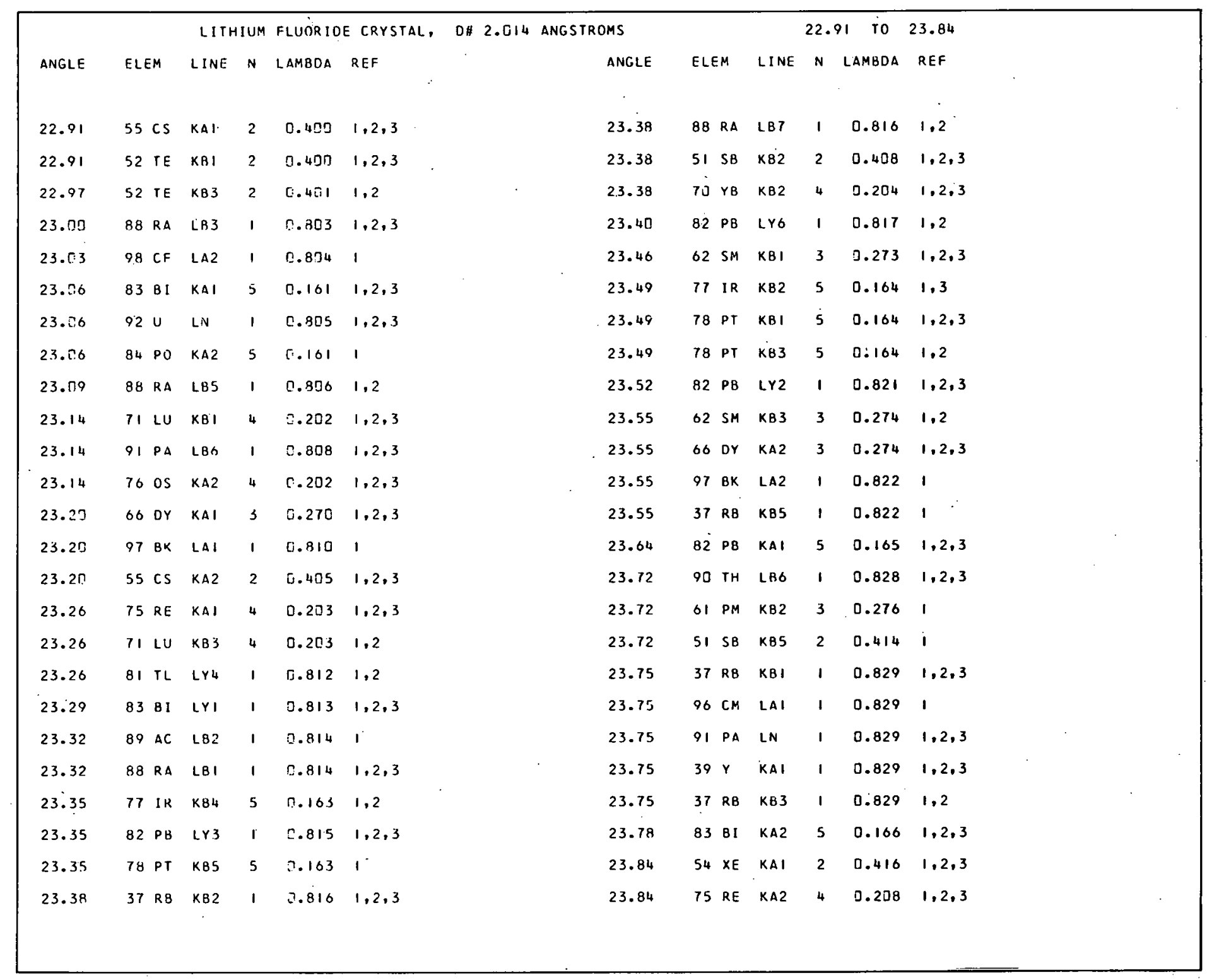




\begin{tabular}{|c|c|c|c|c|c|c|c|c|c|c|c|c|c|c|}
\hline & & LITH & ItU:4 & FLUORIDE & E CRYSTAL, & D\# 2.014 & ANGSTROMS & & & & 23. & 87 TO & 24.95 & \\
\hline ANCLE & ELEM & LINE & $N$ & LAMBDA & REF & & ANGLE & ELE & & L I NE & $\mathbf{N}$ & LAMBDA & REF & \\
\hline 23.87 & $35 Y$ & KA2 & 1 & 0.833 & $1,2,3$ & & 24.34 & 65 & TB & KA2 & 3 & 0.283 & $1,2,3$ & \\
\hline 23.90 & $51 \mathrm{SB}$ & KBI & 2 & 0.417 & $1,2,3$ & & 24.34 & 61 & PM & KB3 & 3 & 0.283 & 2 & \\
\hline 23.93 & 77 IR & KBS & 5 & 0.167 & 1 & & 24.36 & 81 & IL & KAI & 5 & 0.170 & $1,2,3$ & \\
\hline 23.93 & $8 E R A$ & LB2 & 1 & 0.835 & $1,2,3$ & & 24.36 & 82 & PB & KA2 & 5 & 0.170 & 1.2 .3 & \\
\hline 23.96 & $7[\mathrm{YB}$ & KBI & 4 & 0.200 & $1,2,3$ & & 24.42 & 50 & SN & KB2 & 2 & 0.426 & $1,2,3$ & \\
\hline 23.96 & $7[$ YB & KB 3 & 4 & 0.209 & 1,2 & $\cdot$ & 24.48 & 90 & TH & LN & 1 & 0.854 & $1,2,3$ & \\
\hline 23.96 & $50 \mathrm{SB}$ & KB3 & 2 & 0.418 & 1,2 & & 24.54 & 74 & $w$ & KA2 & 4 & 0.214 & $1,2,3$ & \\
\hline 23.96 & $74 W$ & KAI & 4 & 0.2009 & $1,2.3$ & & 24.63 & 60 & ND & KB2 & 3 & 0.286 & $1,2,3$ & \\
\hline 23.99 & $6 \subseteq T B$ & KAI & 3 & 0.279 & $1,2,3$ & & 24.60 & 87 & $F R$ & LB2 & 1 & 0.853 & $1,2,3$ & \\
\hline 24.04 & $8 \geq B \mathrm{I}$ & LYS & 1 & 0.8399 & 1,2 & & 24.66 & 95 & $A M$ & LA2 & 1 & 0.860 & 1,3 & \\
\hline 24.04 & $8[\mathrm{HG}$ & LY4 & 1 & 0.839 & 1,2 & & 24.66 & 73 & TA & KAI & 4 & 0.215 & $1,2,3$ & · \\
\hline 24.97 & $8 \overline{\angle P B}$ & LYI & 1 & 0.840 & $1,2,3$ & & 24.66 & 69 & $T M$ & KBI & 4 & 0.215 & $1,2,3$ & \\
\hline 24.07 & $87 \mathrm{FR}$ & LBI & 1 & 0.840 & $1,2,3$ & & 24.77 & 69 & rM & KB 3 & 4 & 0.215 & 1,2 & \\
\hline 24.07 & $54 \times E$ & KA2 & 2 & 0.420 & $1,2,3$ & & 24.77 & 50 & SN & KB5 & 2 & 0.432 & 1 & \\
\hline 24.07 & $68 \mathrm{TM}$ & KB2 & 4 & 0.210 & 1 & & 24.77 & 64 & GD & KAI & 3 & 0.288 & $1,2,3$ & \\
\hline 24.10 & QE $\mathrm{CH}$ & LA2 & 1 & 0.84 .1 & 1 & & 24.83 & 36 & $K R$ & $K B 2$ & 1 & 0.866 & $1,2,3$ & \\
\hline 24.10 & $8 \mathrm{I} \cdot \mathrm{TL}$ & LY3 & 1 & 0.84 .1 & $1,2.3$ & & 24.83 & 36 & KR & KB4 & 1 & 0.866 & 2 & \\
\hline 24.10 & $8 \mathrm{RA}$ & LB4 & 1 & 0.84 .1 & $1,2,3$ & & 24.83 & 86 & $\mathrm{RN}$ & LBI & 1 & 0.866 & 1 & \\
\hline 24.19 & $81 \mathrm{TL}$ & LYo & 1 & 0.844 & 1,2 & & 24.83 & 53 & I & KAI & 2 & 0.433 & $1,2,3$ & \\
\hline 24.22 & $7 E$ os & KB2 2 & 5 & 0.169 & 1,3 & & 24.83 & 82 & PB & LY5 & 1 & 0.866 & 1,2 & \\
\hline 24.22 & $7 T$ IR & KB3 & 5 & 0.169 & 1,2 & & 24.89 & 94 & PU & LAI & 1 & 0.868 & 1 & \\
\hline 24.22 & 77 IR & KBI & 5 & 0.168 & $1,2,3$ & & 24.89 & 81 & TL & LYI & 1 & 0.868 & $1,2,3$ & \\
\hline 24.25 & OI PM & KBI & 3 & C:. 282 & $1,2,3$ & & 24.89 & 68 & ER & $k 62$ & 4 & 0.217 & $1,2,3$ & \\
\hline 24.31 & $81 \mathrm{TL}$ & $\operatorname{LY} 2$ & 1 & {$[.849$} & $1,2,3$ & & 24.92 & 80 & HG & $\operatorname{Lr} 3$ & 1 & 0.860 & $1,2,3$ & \\
\hline 24.31 & $95 \mathrm{AM}$ & LAI & 1 & 6.848 & 1,3 & & 24.95 & 76 & os & KEI & 5 & 0.174 & $1,2,3$ & \\
\hline
\end{tabular}




\begin{tabular}{|c|c|c|c|c|c|c|c|c|c|c|c|c|c|c|}
\hline & & LITH & [I]N & FLUOR ID & E CRYSTAL, & $D \# 2.014$ & ANGSTROMS & & & & 24.9 & 95 TO & 26.03 & \\
\hline ANGLE. & ELEM & LINE & is & LAMBDA & REF & . & ANGLE & ELE & & LINE & $N$ & LAMBDA & REF & \\
\hline 24.95 & 76 OS & KB3 & 5 & 0.474 & 1.2 & & 25.59 & 68 & ER & K83 & 4 & 0.223 & 1,2 & \\
\hline 24.95 & 75 RE & $\mathrm{K} 82$ & 5 & $\pi .174$ & 1,3 & & 25.59 & 68 & $E R$ & KBI & 4 & 0.223 & $1,2,3$ & \\
\hline 24.95 & $50 \mathrm{SN}$ & KBI & 2 & 0.435 & $1,2,3$ & & 25.65 & 85 & $\Delta \mathrm{r}$ & LBI & 1 & 0.894 & 1 & \\
\hline 24.98 & $36 \mathrm{KR}$ & KB5 & 1 & 0.871 & 1.2 & & 25.68 & 81 & $\mathrm{IL}$ & LY5 & 1 & 0.895 & 1.2 & \\
\hline 24.98 & $8 B R A$ & LB6 & 1 & 0.871 & $1,2,3$ & & 25.68 & 74 & $w$ & KB2 & 5 & 0.179 & $1,2,3$ & \\
\hline חת. & $50 \mathrm{SN}$ & $K \forall 3$ & 2 & 0.436 & 1,2 & & 25.68 & 74 & $H$ & KB4 & 5 & 0.179 & 1 & \\
\hline 25.03 & $80 \mathrm{HG}$ & LYS & 1 & 0.873 & 1,2 & & 25.68 & 75 & RE & KB3 & 5 & 0.179 & 1.2 & \\
\hline 25.09 & $80 \mathrm{HG}$ & LY2 & 1 & 0.875 & $1,2,3$ & & 25.68 & 75 & RE & $K 81$ & 5 & 0.179 & $1,2,3$ & \\
\hline 25.09 & $385 R$ & KAI & 1 & 0.875 & $1,2,3$ & & 25.70 & 67 & HO & $K B 2$ & 4 & 0.224 & 1 & \\
\hline 25.09 & $80 \mathrm{HG}$ & KAI & 5 & 0.175 & 1 & & 25.73 & 78 & $\rho_{T}$ & LY4 & 1 & 0.897 & 1.2 & \\
\hline 25.09 & BI TL & KA2 & 5 & 0.175 & $1,2,3$ & & 25.73 & 80 & $\mathrm{HG}$ & LYI & 1 & 0.897 & $1,2,3$ & \\
\hline 25.12 & 531 & KA2 & 2 & 0.438 & $1,2,3$ & & 25.73 & 63 & EU & KAI & 3 & 0.299 & $1,2,3$ & \\
\hline 25.18 & $36 \mathrm{KR}$ & KBI & 1 & 0.878 & $1,2,3$ & & 25.76 & 83 & BI & LB9 & 1 & 0.898 & 1.2 & \\
\hline 25.21 & $64 G D$ & KA2 & 3 & 0.293 & $1,2,3$ & & 25.76 & 79 & $\Delta U$ & $1 Y 3$ & 1 & 0.898 & $1,2,3$ & \\
\hline 25.21 & $36 \mathrm{KR}$ & KB 3 & 1 & 0.879 & 1,2 & & 25.79 & 84 & Po & LB5 & 1 & 0.899 & 1,2 & \\
\hline 25.21 & $38 \mathrm{SR}$ & KA2 & 1 & 0.879 & $1,2,3$ & & 25.82 & 79 & AU & KAI & 5 & 0.180 & $1,2,3$ & \\
\hline 25.21 & 60 ND & KBI & 3 & 0.293 & $1,2,3$ & & 25.82 & 80 & HG & KA2 & 5 & 0.180 & 1 & \\
\hline 25.24 & $73 \mathrm{IA}$ & KA2 & 4 & D. 220 & $1,2,3$ & & 25.85 & 93 & NP & LA2 & 1 & 0.901 & 1 & \\
\hline 25.24 & $94 \mathrm{PU}$ & LA2 & 1 & 0.880 & 1 & & 25.88 & 49 & IN & $K 85$ & 2 & 0.451 & 1 & \\
\hline 25.27 & $86 \mathrm{RN}$ & LB2 & 1 & $r_{j} .881$ & $!$ & & 25.88 & 52 & $\mathrm{TE}$ & KAI & 2 & 0.451 & $1,2,3$ & \\
\hline 25.30 & 60 ND & $K B 3$ & 3 & 0.294 & 1,2 & & 25.91 & 79 & $A U$ & LYo & 1 & 0.903 & 1,2 & \\
\hline 25.47 & $72 \mathrm{HF}$ & KAI & 4 & 0.222 & $1,2,3$ & & 25.94 & 79 & $A U$ & $\operatorname{LY} 2$ & 1 & 0.904 & $1,2,3$ & \\
\hline 25.50 & $93 \mathrm{NP}$ & LAI & 1 & 0.889 & $1,2,3$ & & 25.97 & 85 & $A T$ & LB2 & 1 & 0.905 & 1 & \\
\hline 25.53 & 49 IN & KB2 & 2 & 0.445 & $1,2,3$ & & 25.97 & 83 & $B 1$ & 1810 & 1 & 0.905 & 1,2 & \\
\hline 25.56 & $59 \mathrm{PR}$ & KB2 & 3 & 0.297 & $1,2,3$ & & 26.03 & 88 & $\mathrm{RA}$ & LN & 1 & 0.997 & $1,2,3$ & \\
\hline
\end{tabular}




\begin{tabular}{|c|c|c|c|c|c|c|c|c|c|c|c|c|c|c|}
\hline & & LITH & IIUM & FLUORID & E CRYSTALL, & DA 2.014 & ANGSTROMS & & & & 26. & Tо & 27.22 & \\
\hline ANGLE & ELE Y & LINE & $\mathbf{N}$ & LAMBDA & REF & & ANGLE. & ELE & EM & LINE & $N$ & LAMBDA & REF & \\
\hline 26.05 & $72 \mathrm{HF}$ & KA2 & 4 & 0.227 & $1,2,3$ & & 26.61 & 62 & SM & KAI & 3 & 0.309 & $1,2,3$ & - \\
\hline 26.08 & $83 \mathrm{EU}$ & KA2 & 3 & 0.303 & $1,2,3$ & & 26.61 & 77 & IR & LY4 & 1 & 0.927 & 1,2 & \\
\hline 26.08 & $84 \mathrm{PO}$ & LB3 & 1 & 0.909 & $1,2,3$ & & 26.61 & 79 & $A U$ & $|Y|$ & 1 & 0.927 & $1,2,3$ & \\
\hline 26.11 & $49 \mathrm{IN}$ & KB3 & 2 & 0.455 & 1,2 & & 26.61 & 82 & PB & LBG & 1 & 0.927 & 1,2 & \\
\hline 26.11 & 49 II N & KB I & 2 & 0.455 & $1,2,3$ & & 26.64 & 66 & DY & $K B Z$ & 4 & 0.232 & $1,2,3$ & \\
\hline 26.14 & $92 \mathrm{U}$ & LAI & 1 & 0.911 & $1,2,3$ & & 26.64 & 78 & PT & LY3 & 1 & 0.928 & $1,2,3$ & \\
\hline 26.17 & $52 \mathrm{TE}$ & KA2 & 2 & 0.456 & $1,2,3$ & & 26.67 & 84 & Po & L.E 2 & 1 & 0.929 & $1,2,3$ & \\
\hline 26.17 & $59 P R$ & KBI & 3 & 0.304 & $1,2,3$ & & $26.7 \pi$ & 37 & $\mathrm{RB}$ & $K A 2$ & 1 & 0.930 & $1,2,3$ & \\
\hline 26.26 & $59 P R$ & KB3 & 3 & 0.305 & 1,2 & & 26.70 & 48 & $C D$ & KB2 & 2 & 0.46 .5 & $2,2,3$ & \\
\hline 26.26 & $74 w$ & KB5 & 5 & 0.183 & 1 & & 26.70 & 78 & PT & KAI & 5 & 0.18 .6 & $1,2,3$ & \\
\hline 26.29 & $71 \mathrm{LU}$ & KAI & 4 & 0.229 & $1,2,3$ & & 26.79 & 91 & PA & LAI & 1 & 0.933 & $1,2,3$ & \\
\hline 26.51 & $67 \mathrm{HO}$ & KBI & 4 & 0.230 & 1 & & 26.79 & 35 & $B R$ & $K B_{1}$ & .1 & 0.933 & $1,2,3$ & \\
\hline 26.41 & $74 W$ & KBI & 5 & 0.184 & $1,2,3$ & & 26.79 & 35 & BR & KB 3 & 1 & 0.933 & 1,2 & \\
\hline 26.41 & $73 \mathrm{rA}$ & KB4 & 5 & 0.184 & 1 & & 26.81 & 78 & $P T$ & LYO & 1 & 0.934 & 1.2 & \\
\hline 26.43 & $35 \mathrm{BR}$ & KB2 & 1 & 0.921 & $1,2,3$ & & 26.81 & 78 & PT & LY2 & 1 & 0.934 & $1,2,3$ & \\
\hline 26.46 & $92 \mathrm{U}$ & LA2 & 1 & 0.922 & $1,2,3$ & & 26.81 & 82 & PB & LBIO & 1 & 0.934 & 1.2 & \\
\hline 26.46 & $84 \mathrm{PO}$ & LBI & 1 & 0.922 & $1,2,3$ & & 26.84 & 83 & B I & LB7 & 1 & 0.935 & 1,2 & \\
\hline 26.52 & $80 \mathrm{HG}$ & LYS & 1 & 0.924 & 1,2 & & 26.87 & 71 & LU & $K A 2$ & 4 & 0.234 & $1,2,3$ & \\
\hline 26.52 & $58 \mathrm{CE}$ & KB2 & 3 & $0.30 \varepsilon$ & $1,2,3$ & & 26.96 & 83 & $\mathrm{BI}$ & LB3 & 1 & 0.939 & $1,2,3$ & \\
\hline 26.55 & 79 AU & KA2 & 5 & c. 185 & $1,2,3$ & & 26.99 & 51 & SB & KAI & 2 & 0.470 & $1,2,3$ & \\
\hline 26.55 & $35 \mathrm{BR}$ & KBS & 1 & 0.925 & 1.2 & & 27.05 & 62 & $S M$ & KAZ & 3 & 0.314 & $1,2,3$ & \\
\hline 26.55 & $73 \mathrm{TA}$ & KB2 & 5 & 0.185 & $1,2,3$ & & 27.14 & 91 & $P A$ & LA2 & 1 & 0.945 & $1,2,3$ & \\
\hline 26.55 & $74 W$ & KB3 & 5 & 0.185 & 1,2 & & 27.14 & 73 & TA & KBS & 5 & 0.189 & 1 & \\
\hline 26.58 & $83 \mathrm{BI}$ & LBS & 1 & 0.920 & 1.2 & & 27.20 & 84 & PO & LB4 & 1 & 0.947 & $1,2,3$ & \\
\hline 26.58 & $37 \mathrm{RB}$ & KAI & 1 & 0.926 & $1,2,3$ & & 27.22 & 66 & DY & KB I & 4 & 0.237 & $1,2,3$ & \\
\hline
\end{tabular}




\begin{tabular}{|c|c|c|c|c|c|c|c|c|c|c|c|c|c|c|}
\hline & & LITH & IUM & FLUOR IDE & E CRYSTIAL, & $D \# 2.014$ & ANGSTROMS & & & & 27. & Tо & 28.28 & \\
\hline ANGLE & ELEM & LINE & N & LAMBDA & REF & & ANGLE & ELE & & LINE & N & LAMBDA & REF & \\
\hline 27.22 & $58 \mathrm{CE}$ & $K B 3$ & 3 & 0.316 & 1,2 & & 27.69 & 81 & $T L$ & $\angle B 10$ & 1 & 0.964 & 1,2 & \\
\hline 27.22 & 70 YB & KAI & 4 & 0.237 & $1,2,3$ & & 27.69 & 70 & YB & KA2 & 4 & 0.241 & $1,2,3$ & \\
\hline $27.2 ?$ & $58 \mathrm{CE}$ & KBI & 3 & 0.316 & $1,2,3$ & & 27.72 & 77 & IR & LY2 & 1 & 0.965 & $1,2,3$ & \\
\hline 27.28 & $73 \mathrm{TA}$ & KBI & 5 & 0.190 & $1,2,3$ & & 27.78 & 77 & IR & LY6 & 1 & 0.967 & 1,2 & \\
\hline 27.28 & $78 \mathrm{PI}$ & KA2 & 5 & 0.190 & $1,2,3$ & & 27.78 & 84 & Po & LB6 & 1 & 0.967 & $1,2,3$ & \\
\hline 27.28 & $48 \mathrm{CD}$ & KBI & 2 & 0.475 & $1,2,3$ & & 27.81 & 90 & $\mathrm{TH}$ & LA2 & 1 & 0.968 & $1,2,3$ & \\
\hline 27.28 & SI SB & KA2 & 2 & 0.475 & $1,2,3$ & & 27.84 & 82 & PB & LB3 & 1 & 0.969 & $1,2,3$ & \\
\hline 27.34 & $48 \mathrm{CD}$ & $K B 3$ & 2 & 0.476 & 1,2 & & 27.99 & 47 & $A G$ & $K B 2$ & 2 & 0.487 & $1,2,3$ & \\
\hline 27.34 & $66 \mathrm{OY}$ & KB3 & 4 & 0.238 & 1,2 & & 28.02 & .61 & $P M$ & KA2 & 3 & 0.325 & $1,2,3$ & \\
\hline 27.34 & $83 \mathrm{BI}$ & LBI & 1 & 0.952 & $1,2,3$ & & 28.04 & 69 & $I M_{i}$ & KAI & 4 & 0.244 & $1,2,3$ & \\
\hline 27.37 & $82 \mathrm{~PB}$ & L85 & 1 & 0.953 & 1.2 & & 28.07 & 83 & B I & 184 & 1 & 0.977 & $1,2,3$ & \\
\hline 27.43 & $72 \mathrm{HF}$ & KB2 & 5 & c.191 & $1,2,3$ & & 28.10 & 57 & LA & $K 85$ & 3 & 0.326 & 1 & \\
\hline 27.43 & 77 IR & KAI & 5 & 0.191 & $1,2,3$ & & 28.16 & 72 & HF & $K B 3$ & 5 & 0.196 & 1,2 & \\
\hline 27.43 & $73 T A$ & K83 & 5 & 0.191 & 1,2 & & 28.16 & 72 & $\mathrm{HF}$ & KBI & 5 & 0.196 & $\cdot 1,2,3$ & \\
\hline 27.43 & $83 \mathrm{BI}$ & $\angle B 2$ & 1 & 0.955 & $1,2,3$ & & 28.16 & 34 & $S E$ & $\mathrm{~K} 82$ & 1 & 0.980 & $1,2,3$ & \\
\hline 27.46 & $90 \mathrm{TH}$ & LAI & 1 & 0.956 & $1,2,3$ & . & 28.16 & 89 & $A C$ & LAI & 1 & 0.980 & 1 & \\
\hline 27.46 & 79. AU & LYS & 1 & 0.956 & 1,2 & & 28.16 & 77 & IR & KA2 & 5 & 0.196 & $1,2,3$ & \\
\hline 27.4 .9 & $81 \mathrm{TL}$ & LBO & 1 & 0.957 & 1,2 & . & 28.16 & 36 & $K R$ & KAI & 1 & 0.980 & $1,2,3$ & . \\
\hline 27.52 & $78 \mathrm{PT}$ & LrI & 1 & 0.958 & $1,2,3$ & & 28.19 & 81 & TL & LBS & 1 & 0.981 & 1,2 & \\
\hline 27.55 & 7605 & $1 Y_{4}$ & 1 & 0.959 & 1,2 & & 28.22 & 50 & SN & KAI & 2 & 0.491 & $1,2,3$ & \\
\hline 27.55 & $77 \mathrm{IR}$ & LY3 & 1 & 0.959 & $1,2,3$ & & 28.22 . & 82 & PB & LBI & 1 & 0.982 & $1,2,3$ & \\
\hline 27.58 & $65 \mathrm{TH}$. & Kв2 & 4 & $r_{i} .240$ & $1,2,3$ & & 28.25 & 82 & PB & LB2 & 1 & 0.983 & $1,2,3$ & \\
\hline 27.58 & $57 \mathrm{LA}$ & KB2 & 3 & $0.32 E$ & $1,2,3$ & & 28.28 & 57 & LA & KBI & 3 & 0.328 & $1,2,3$ & \\
\hline 27.63 & $82 \mathrm{~PB}$ & LBT & 1 & 0.962 & 1,2 & & 28.28 & 65 & $T_{B}$ & KB3 & 4 & 0.246 & 1,2 & \\
\hline 27.66 & $61 P M$ & KAI & 3 & 0.321 & $1,2,3$ & & 28.28 & 36 & KR & KA2 & 1 & 0.984 & $1,2,3$ & \\
\hline
\end{tabular}




\begin{tabular}{|c|c|c|c|c|c|c|c|c|c|c|c|c|}
\hline \multirow[b]{2}{*}{ ANGLE } & \multirow[b]{2}{*}{ ELEM } & \multicolumn{2}{|c|}{ LITHIUM } & \multicolumn{2}{|c|}{ FLUORIDE CRYSTAL, } & \multirow[t]{2}{*}{ D\#2.014 } & \multicolumn{3}{|l|}{ ANCSTROMS } & \multicolumn{3}{|c|}{28.28 TO 29.42} \\
\hline & & LINE & $N$ & LAMBDA & REF & & ANGLE & ELEM & LINE & $N$ & LAMBDA & REF \\
\hline 28.28 & $34 S E$ & KB5 & 1 & 0.988 & 1,2 & & 28.78 & 7605 & LYo & 1 & 1.001 & 1,2 \\
\hline 28.28 & $65 \mathrm{~TB}$ & $\mathrm{KBI}$ & 4 & 0.240 & $1,2,3$ & & 28.78 & $81 \mathrm{TL}$ & LB3 & 1 & 1.001 & $1,2,3$ \\
\hline 28.31. & $760 \mathrm{OS}$ & KAI & 5 & $0.19^{\circ}$ & $1,2,3$ & & 28.90 & $88 R A$ & LAI & 1 & 1.005 & $1,2,3$ \\
\hline 28.31 & $71 \mathrm{LU}$ & KB2 & 5 & $0.19^{\circ}$ & $1,2,3$ & & 28.95 & $82 \mathrm{~PB}$ & L.84 & 1 & 1.007 & $1,2,3$ \\
\hline 28.34 & $47 A G$ & K85 & 2 & 0.493 & 1 & & 28.98 & 68 ER & KAI & 4 & 0.252 & $1,2,3$ \\
\hline 28.34 & $80 \mathrm{HG}$ & LB9 & 1 & 0.98 .5 & 1,2 & & 28.98 & 60 ND & $K A .2$ & 3 & 0.336 & $1,2,3$ \\
\hline 28.37 & $57 \mathrm{LA}$ & $K B 3$ & 3 & 0.320 & 1,2 & & 29.04 & $81 \mathrm{TL}$ & LE. 2 & 1 & 1.010 & 1.2 .3 \\
\hline 28.40 & 78. PT & LYS & 1 & 0.983 & 1,2 & & 29.04 & $80 \mathrm{HG}$ & L.65 & 1 & 1.0 .10 & 1,2 \\
\hline 28.46 & $50 \mathrm{SN}$. & KA2 & 2 & 0.495 & 1,$2 ; 3$ & & 29.04 & 76 os & $\mathrm{KH2}$ & 5 & 0.202 & $1,2,3$ \\
\hline 28.46 & $81 \mathrm{TL}$ & LB7 & 1 & {$[.99 .7$} & 1,2 & & 29.04 & $71 \mathrm{LU}$ & KBI & 5 & 0.202 & $1,2,3$ \\
\hline 28.48 & $7 T$ IR & LYI & 1 & $0.99 ! 1$ & $1,2,3$ & & 29.16 & $50 \mathrm{BA}$ & $K 85$ & 3 & 0.338 & 1 \\
\hline 28.51 & $89 \mathrm{AC}$ & LA2 & 1 & 0.992 & 1 & & 29.19 & $71 \mathrm{LU}$ & $K 83$ & 5 & 0.203 & 1,2 \\
\hline 28.51 & $34 \mathrm{SE}$ & KBI & 1 & 0.892 & $1,2,3$ & & 29.19 & $75 \mathrm{RE}$ & KAI & 5 & 0.203 & $1,2.3$ \\
\hline 28.51 & TE OS & LY3 & 1 & 0.992 & $1,2,3$ & & 29.19 & $81 \mathrm{TL}$ & LBI & 1 & 1.015 & $1,2,3$ \\
\hline 28.51 & $7 \subseteq R E$ & $\mathrm{Lr}_{4}$ & 1 & 0.992 & 1,2 & & 29.22 & $88 \mathrm{RA}$ & LA2 & 1 & 1.016 & $1,2,3$ \\
\hline 28.51 & $64 G D$ & KB2 & 4 & 0.248 & $1,2,3$ & & 29.22 & $64 \mathrm{GD}$ & KBI & 4 & 0.254 & $1,2,3$ \\
\hline 28.54 & $8 \equiv B I$ & LB6 & 1 & 0.993 & $1,2,3$ & & 29.31 & $80 \mathrm{HG}$ & LB? & 1 & 1.019 & 1,2 \\
\hline 28.54 & $3 L \mathrm{SE}$ & KB3 & 1 & 3.993 & 1,2 & & 29.34 & $46 \mathrm{PD}$ & KB2 & 2 & 0.510 & $1,2,3$ \\
\hline 28.57 & $4 T A G$ & KBI & 2 & 0.487 & $1,2,3$ & & 29.34 & $70 \mathrm{YB}$ & $k 32$ & 5 & 0.204 & $1,2,3$ \\
\hline $28.6 ?$ & $4: A G$ & KB3 & 2 & 0.458 & 1,2 & & 29.34 & $64 \mathrm{GD}$ & KB3 & 4 & 0.255 & 1,2 \\
\hline 28.63 & 60 NO & KAI & 3 & $0.3 \geq 2$ & $1,2,3$ & & 29.37 & $79 \mathrm{AU}$ & LB9 & 1 & 1.021 & 1,2 \\
\hline 28.63 & $80 \mathrm{HG}$ & 2810 & 1 & 0.956 & 1,2 & & 29.37 & $82 \mathrm{~PB}$ & LBG & 1 & 1.021 & $1,2,3$ \\
\hline 28.63 & $0^{\circ} \mathrm{TMM}$ & KAZ & 4 & $0.2<9$ & $1,2,3$ & & 29.40 & 77 IR & LYS & 1 & 1.022 & 1,2 \\
\hline 28.69 & 70 OS & LY2 & 1 & 0.998 & $1,2,3$ & & 29.42 & $56 \mathrm{BA}$ & $K B 3$ & 3 & 0.341 & 1,2 \\
\hline 28.72 & Si $8 A$ & KB2 & 3 & 1.333 & $1,2,3$ & & 29.42 & $56 \mathrm{BA}$ & KBI & 3 & 0.341 & $1,2,3$ \\
\hline
\end{tabular}




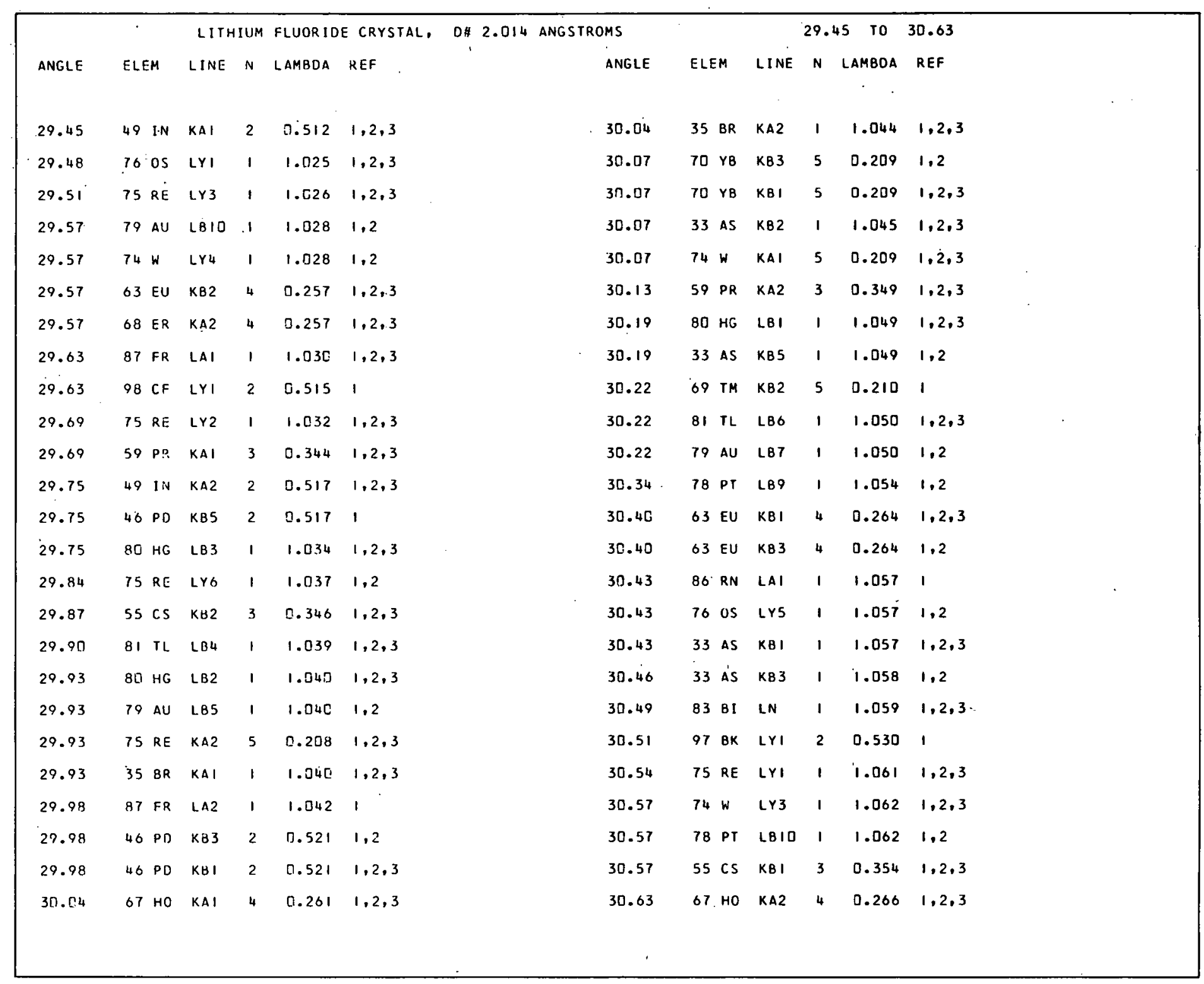




\begin{tabular}{|c|c|c|c|c|c|c|c|c|c|c|c|c|c|c|c|}
\hline & & LITH & IUM & FLUORIOE & E CRYSTAL, & DH & 2.014 & ANGSTROMS & & & & 30. & 63 T0 & 31.84 & \\
\hline ANGLE & ELEM & LINE & $N$ & LAMBDA & REF & & & ANGLE & ELE & & LINE & N & LAMEDA & REF & \\
\hline 30.63 & $625 M$ & $\mathrm{KB2}$ & 4 & $C .265$ & $1,2,3$ & & & 31.25 & 68 & $E R$ & KB2 & 5 & 0.217 & $1,2,3$ & \\
\hline 30.66 & $55 \mathrm{cs}$ & K83 & 3 & {$[.355$} & 1,2 & & & 31.25 & 85 & $A T$ & LAI & 1 & 1.085 & 1 & \\
\hline 30.66 & $73 \mathrm{TA}$ & LYH & 1 & 1.0 .65 & 1,2 & & & 31.28 & 58 & CE & KA2 & 3 & 0.362 & $1,2,3$ & \\
\hline 30.72 & $92 \mathrm{U}$ & LL & 1 & 1.067 & 2,3 & & & 31.40 & 77 & IR & 289 & 1 & 1.090 & 1.2 & \\
\hline 30.75 & 78 AU & LB 3 & 1 & 1.968 & $1,2,3$ & & & 31.43 & 91 & PA & LL & 1 & 1.091 & $1,2,3$ & \\
\hline 30.75 & $74 \mathrm{~W}$ & LY2 & 1 & 1.068 & $1,2,3$ & & . & 31.46 & 82 & PB & LN & 1 & 1.092 & $1,2,3$ & \\
\hline 30.78 & $8 E \mathrm{RN}$ & LA2 & 1 & 1.069 & 1 & & & 31.46 & 96 & CM & LYYI & 2 & 0.546 & $\mathbf{I}$ & \\
\hline 30.81 & $4 \varepsilon C D$ & KAI & 2 & 0.535 & $1,2,3$ & & & 31.46 & 45 & RH & KB3 & 2 & 0.546 & 1,2 & \\
\hline 30.81 & $7 * w$ & KA2 & 5 & 0.214 & $1,2,3$ & & & 31.46 & 45 & RH & KBI & 2 & 0.546 & $1,2,3$ & \\
\hline 30.81. & $4 \equiv R H$ & KB2 & 2 , & 0.535 & $1,2,3$ & & & 31.46 & 62 & SM & KBI & 4 & 0.273 & $1,2,3$ & 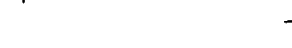 \\
\hline 30.81 & $7 \equiv A U$ & L82 & 1 & 1.070 & $1,2,3$ & & & 31.52 & 75 & RE & Lrs & 1 & 1.094 & 1,2 & \\
\hline 30.84 & $5 E C E$ & KAI & 3 & 0.357 & $1,2,3$ & & & 31.58 & 62 & SM & Kз3 & 4 & 0.274 & 1,2 & \\
\hline 30.87 & $7 E P T$ & LBS & 1 & 1.072 & 1,2 & & & 31.58 & 66 & or & KA2 & 4 & 0.274 & $1,2,3$ & \\
\hline 30.87 & $89 \mathrm{HG}$ & LB4 & 1 & 1.072 & $1,2,3$ & & & 31.81 & 85 & $A T$ & $\operatorname{LA2}$ & $\mathbf{I}$ & 1.097 & 1 & \\
\hline 30.9 .3 & $7 \mathrm{~K} \mathrm{~W}$ & LYG & 1 & 1.074 & 1,2 & & & 31.61 & 77 & IR & LBIO & 1 & 1.097 & 1,2 & \\
\hline $30.9 \mathrm{c}$ & $6^{\circ} \mathrm{TM}$ & KBI & 5 & ח.2 15 & $1,2,3$ & & & 31.67 & 74 & $H$ & LYI & 1 & 1.099 & $1,2,3$ & \\
\hline $30.9 t$ & $7 B$ TA & KAI & 5 & 5.215 & $1,2,3$ & & & 31.67 & 73 & TA & LY3 & 1 & 1.099 & $1,2,3$ & \\
\hline 31.05 & $48 \mathrm{CD}$ & $K A \bar{C}$ & 2 & 0.559 & $1,2,3$ & & & 31.70 & 73 & TA & KA2 & 5 & 0.220 & $1,2,3$ & \\
\hline 31.15 & $8 \mathrm{C} \mathrm{HG}$ & $\angle B 6$ & 1 & 1.080 & $1,2,3$ & & & 31.75 & 78 & PT & LB 2 & 1 & 1.102 & $1,2,3$ & \\
\hline 31.15 & 60 OY & KAI & 4 & 0.270 & $1,2,3$ & & & 31.78 & 72 & $\mathrm{HF}$ & $\mathrm{Lr}_{4}$ & 1 & 1.103 & 1,2 & \\
\hline 31.10 & $54 \times E$ & KB2 & 3 & 0.300 & $1,2,3$ & & & 31.81 & 54 & $X E$ & $K B I$ & 3 & 0.368 & $1,2,3$ & \\
\hline 31.10 & $62 T M$ & $\mathrm{~KB} 3$ & 5 & 3.216 & 1,2 & & & 31.81 & 78 & PT & LB3 & 1 & 1.104 & $1,2,3$ & \\
\hline 31.16 & $45 \mathrm{RH}$ & KBS & 2 & 2.5 上 1 & 1 & & & 31.81 & 61 & $P M$ & $K B 2$ & 4. & 0.270 & 1 & \\
\hline 31.10 & $7.3 \mathrm{PT}$ & 187 & 1 & 1.082 & 1.2 & & & 31.84 & 34 & SE & KAI & 1 & 1.105 & $1,2,3$ & \\
\hline 31.22 & $7.8 \mathrm{AU}$ & LB! & 1 & 1.084 & $1,2,3$ & & & 31.84 & 73 & TA & $.2 Y 2$ & 1 & 1.105 & $1,2,3$ & \\
\hline
\end{tabular}




\begin{tabular}{|c|c|c|c|c|c|c|c|c|c|c|c|c|c|c|c|}
\hline & & LITH & IUM & FLUORIDE & E CRYSTAL, & $\mathrm{OH}$ & 2.014 & ANGSTROMS & & & & 31.8 & 87 TO & 33.15 & \\
\hline ANGLE & ELEM & LINE & $N$ & LAMBDA & REF & & & ANGLE & ELE & & LINE & $N$ & LAMBDA & REF & \\
\hline 31.87 & $77 \mathrm{IR}$ & LBS & 1 & 1.106 & 1,2 & & & 32.52 & .47 & $\Delta G$ & KA2 & 2 & 0.564 & $1,2,3$ & \\
\hline 31.90 & $79 \mathrm{AU}$ & LB 4 & 1 & 1.107 & $1,2,3$ & & & 32.52 & 81 & TL & LN & 1 & 1.128 & $1,2,3$ & \\
\hline 31.96 & $34 S E$ & KA2 & 1. & 1.109 & $1,2,3$ & & & 32.55 & 32 & GE & KB:I & 1 & 1.129 & $1,2,3$ & \\
\hline 31.99 & $72 \mathrm{HF}$ & KAI & 5 & 0.222 & $1,2,3$ & & & 32.55 & 32 & GE & KB 3 & 1 & 1.129 & 1,2 & \\
\hline 32.02 & $79 \mathrm{AU}$ & LBO & 1 & 1.111 & $1,2,3$ & & & 32.64 & 74 & $w$ & LYS & 1 & 1.132 & 1,2 & \\
\hline 32.7 .8 & $57 \mathrm{LA}$ & KAI & 3 & 0.371 & $1,2,3$ & & & 32.64 & 61 & PM & KB 3 & 4 & 0.283 & 2 & \\
\hline 32.11 & $73 \mathrm{TA}$ & LYO & 1 & 1.114 & 1,2 & & & 32.64 & 65 & TB & KA2 & 4 & 0.283 & $1,2,3$ & \\
\hline 32.11 & $84 \mathrm{PO}$ & LAI & 1 & 1.114 & $1,2,3$ & & & 32.67 & 76 & os & $\angle B 10$ & 1 & 1.133 & 1.2 & \\
\hline 32.14 & 77 IR & $\angle B T$ & 1 & 1.115 & 1,2 & & & 32.73 & 77 & IR & LB2 & 1 & 1.135 & $1,2,3$ & \\
\hline 32.14 & $90 \mathrm{TH}$ & LL & 1 & 1.115 & $1,2,3$ & & & 32.73 & 72 & $\mathrm{HF}$ & KA2 & 5 & 0.227 & $1,2,3$ & \\
\hline 32.14 & $68 E R$ & KE3 & 5 & 0.223 & 1.2 & & & 32.76 & 44 & RU & KB5 & 2 & 0.568 & 1 & \\
\hline 32.14 & $68 \mathrm{ER}$ & KBI & 5 & 0.223 & $1,2,3$ & & & 32.82 & 73 & iA & $|y|$ & 1 & 1.138 & $1,2,3$ & \\
\hline 32.17 & $65 \mathrm{rB}$ & KAI & 4 & 0.279 & $1,2,3$ & & & 32.82 & 721 & $\mathrm{HF}$ & LY3 & 1 & 1.138 & 1.2 .3 & \\
\hline $32.2 \pi$ & $32 \mathrm{GE}$ & KB2 & 1 & 1.117 & $1,2,3$ & & & 32.88 & 76 & os & ¿B5 & 1 & 1.140 & 1,2 & \\
\hline 32.23 & $47 \Delta G$ & KAI & 2 & 0.559 & $1,2,3$ & & & 32.91 & 77 & IR & LB3 & 1 & 1.141 & $1,2,3$ & \\
\hline 32.26 & $32 \mathrm{GE}$ & KB5 & 1 & 1.119 & 1,2 & & & 32.94 & 78 & PT & 284 & 1 & 1.142 & $1,2,3$ & \\
\hline 32.29 & $67 \mathrm{HO}$ & KB 2 & $s$ & 0.224 & 1 & & & 32.97 & 71 & LU & $2 Y_{4}$ & 1 & 1.143 & 1 & \\
\hline 32.29 & $78 \mathrm{PT}$ & LBI & 1 & 1.120 & $1,2,3$ & & & 33.00 & 83 & B I & LAI & 1 & 1.144 & $1,2.3$ & \\
\hline 32.41 & $95 \Delta M$ & $|Y|$ & 2 & 0.562 & 1,3 & & & 33.00 & 44 & RU & KBI & 2 & 0.572 & $1,2,3$ & \\
\hline 32.41 & $44 \mathrm{RU}$ & KB2 & 2 & 8.562 & $1,2,3$ & & & 33.00 & 78 & PT & LBG & 1 & 1.144 & $1,2,3$ & \\
\hline 32.44 & $57 \mathrm{LA}$ & KA2 & 3 & 0.375 & $1,2,3$ & & & 33.00 & 72 & $\mathrm{HF}$ & LY2 & 1 & 1.144 & $1,2,3$ & \\
\hline 32.44 & 531 & $K \forall 2$ & 3 & $r_{1} .375$ & $1,2,3$ & & & 33.00 & 65 & ND & KB 2 & 4 & 0.286 & $1,2,3$ & \\
\hline 32.47 & 76 OS & LBQ & 1 & 1.126 & 1.2 & & & 33.03 & 71 & LU & KAI & 5 & 0.229 & $1,2,3$ & \\
\hline 32.47 & $84 P O$ & LA2 & 1 & 1.126 & $1,2,3$ & & & 33.06 & 44 & RU & KB3 & 2 & 0.573 & 1,2 & \\
\hline 32.52 & $61 \mathrm{PM}$ & KBI & 4 & $\because 282$ & $1,2,3$ & & & 33.15 & 76 & os & $\angle B 7$ & 1 & 1.149 & 1.2 & \\
\hline
\end{tabular}




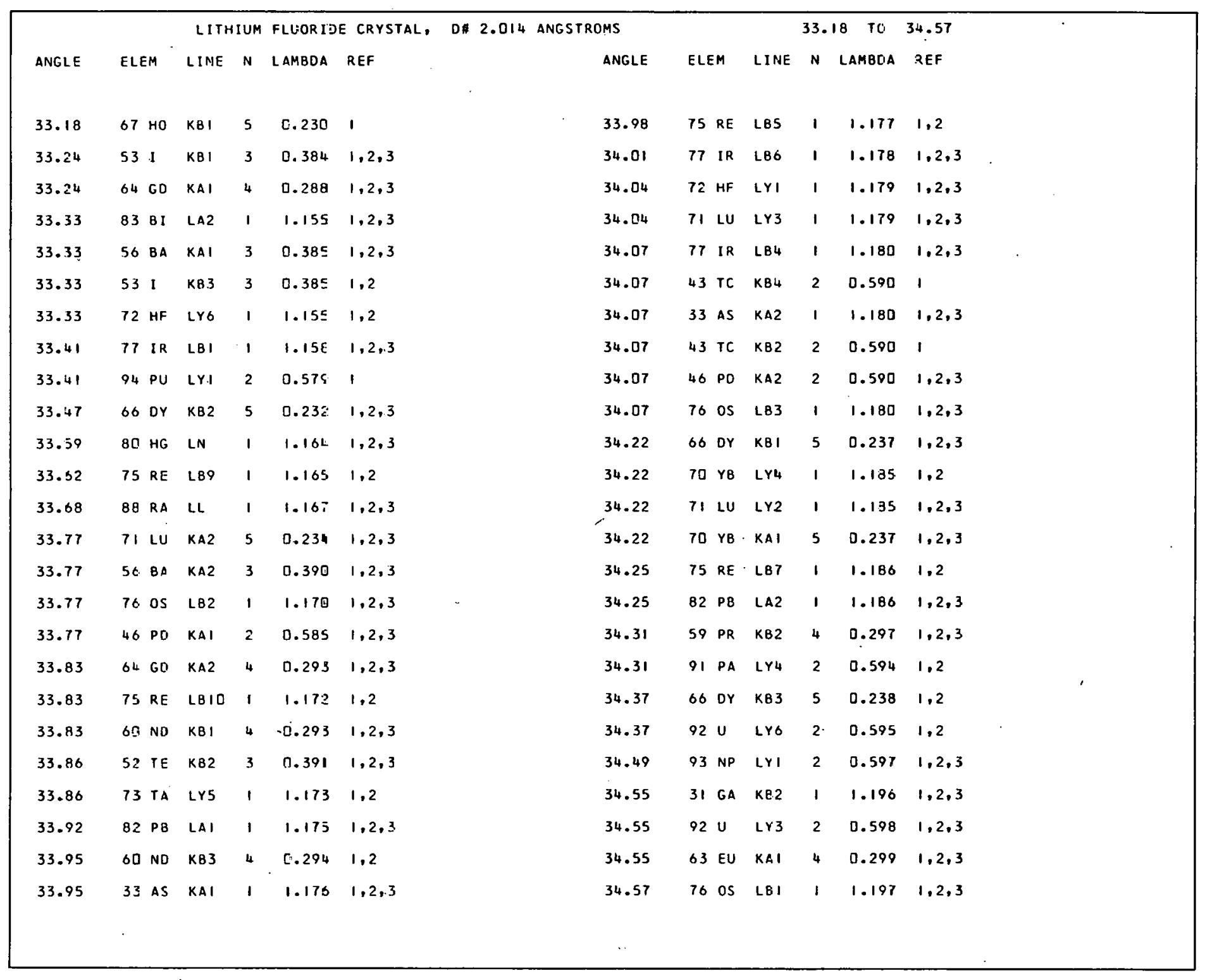




\begin{tabular}{|c|c|c|c|c|c|c|c|c|c|c|c|c|c|}
\hline \multirow[b]{2}{*}{ ANGLE } & \multirow[b]{2}{*}{ ELEM } & \multicolumn{2}{|c|}{ LITHIUM } & \multirow{2}{*}{$\begin{array}{l}\text { FLUOR IDE } \\
\text { LAMBDA }\end{array}$} & \multirow{2}{*}{$\begin{array}{l}\text { E CRYSTAL, } \\
\text { REF }\end{array}$} & \multirow[t]{2}{*}{ D\# 2.014} & \multicolumn{4}{|l|}{ ANGSTROMS } & \multicolumn{3}{|c|}{$34.57 \quad 10 \cdot 35.92$} \\
\hline & & LINE & $N$ & & & & ANGLE & ELE & & LINE & N & LAMBDA & REF \\
\hline 34.57 & $31 G A$ & KBS & 1 & 1.197 & 1.2 & & 35.23 & 81 & TL & LA2 & 1 & 1.219 & $1,2,3$ \\
\hline $34.6 !$ & $71 \mathrm{LU}$ & LYG & 1 & 1.198 & 1,2 & & 35.26 & 59 & PR & KB 3 & 4 & 0.305 & 1,2 \\
\hline 34.66 & $55 \mathrm{cs}$ & KAI & 3 & 9.400 & $1,2,3$ & & 35.26 & 69 & TM & KAI & 5 & 0.244 & $1,2,3$ \\
\hline 34.66 & 65 TB & KB2 & 5 & 0.240 & $1,2,3$ & & 35.26 & 75 & RE & LB3 & 1 & 1.220 & $1,2,3$ \\
\hline 34.66 & $S 2 T E$ & KBI & 3 & 0.400 & $1,2,3$ & & 35.32 & 90 & TH & $L Y_{4}$ & 2 & 0.611 & 1,2 \\
\hline 34.72 & $43 \mathrm{TC}$ & KB3 & 2 & 8.601 & 1,2 & & 35.32 & 71 & LU & LYI & 1 & 1.222 & $1,2,3$ \\
\hline 34.75 & $52 \mathrm{TE}$ & XB3 & 3 & 0.401 & 1,2 & & 35.32 & 70 & YB & $\operatorname{Lr} 3$ & 1 & 1.222 & $1,2,3$ \\
\hline 34.75 & $73 \mathrm{AU}$ & LN & 1 & 1.203 & $1,2,3$ & & 35.38 & 74 & W & LB7 & 1 & 1.224 & 1,2 \\
\hline 34.78 & $74 \mathrm{~W}$ & $\angle B 9$ & 1 & 1.204 & 1,2 & & 35.38 & 51 & SB & KB2 & 3 & 0.408 & $1,2,3$ \\
\hline 34.78 & $43 \mathrm{TC}$ & KBI & 2 & 0.602 & $1,2,3$ & & 35.44 & 91 & PA & LYo & 2 & 0.613 & 1,2 \\
\hline 34.81 & $70 Y 8$ & KA2 & 5 & 0.241 & $1,2,3$ & & 35.44 & 45 & RH & KAI & 2 & 0.613 & $1,2,3$ \\
\hline 34.84 & $98 \mathrm{CF}$ & LBI & 2 & 0.60 .3 & 1 & & 35.53 & 69 & $T M$ & LY4 & 1 & 1.229 & 1 \\
\hline 34.87 & $75 \mathrm{RE}$ & LB2 & 1 & 1.207 & $1,2,3$ & & 35.53 & 70 & YB & LY2 & 1 & 1.229 & $1,2,3$ \\
\hline 34.87 & $81 \mathrm{TL}$ & EAI & 1 & 1.207 & $1,2,3$ & & 35.56 & 92 & u & $(Y)$ & 2 & 0.615 & $1,2,3$ \\
\hline 34.90 & $31 G A$ & KB3 & 1 & 1.270 & 1.2 & & 35.56 & 65 & TB & KB3 & 5 & 0.246 & 1,2 \\
\hline 34.90 & $316 A$ & KBI & 1 & 1.208 & $1,2,3$ & & 35.56 & 65 & TB & KBI & 5 & 0.246 & $1,2,3$ \\
\hline 34.96 & $92 \mathrm{U}$ & LYZ & 2 & 0.605 & $1,2,3$ & & 35.62 & 58 & CE & $K B 2$ & 4 & 0.308 & $1,2,3$ \\
\hline $35^{\circ} .92$ & $63 \mathrm{EU}$ & KA2 & 4 & 0.303 & $1,2,3$ & & 35.68 & 91 & PA & LY3 & 2 & 0.617 & $1,2,3$ \\
\hline $35 . ? 2$ & $74 \mathrm{~W}$ & $2 B 10$ & 1 & 1.212 & 1,2 & & 35.74 & 62 & $S M$ & KAI & 4 & 0.309 & $1,2,3$ \\
\hline 35.05 & 76 OS & L86 & 1 & 1.213 & $1,2,3$ & & 35.74 & 45 & $\mathrm{RH}$ & KA2 & 2 & 0.618 & $1,2,3$ \\
\hline 35.11 & $72 \mathrm{HF}$ & LYS & 1 & 1.215 & 1,2 & & 35.83 & 75 & RE & $\lfloor 81$ & 1 & 1.239 & $1,2,3$ \\
\hline 35.11 & $74 \mathrm{~W}$ & LBS & 1 & 1.215 & 1,2 & & 35.86 & 42 & MO & KB4 & 2 & 0.620 & 2 \\
\hline 35.11 & $55 \mathrm{cs}$ & KA2 & 3 & 0.405 & $1,2,3$ & & 35.86 & 64 & GD & $K B 2$ & 5 & 0.248 & $1,2,3$ \\
\hline 35.14 & $59 \mathrm{PR}$ & KBI & 4 & 0.304 & $1,2,3$ & & 35.89 & 80 & HG & LAI & 1 & 1.241 & $1,2,3$ \\
\hline 35.20 & 76 os & LB4 & 1 & 1.218 & $1,2,3$ & & 35.92 & 51 & SB & KBS & 3 & 0.414 & 1 \\
\hline
\end{tabular}




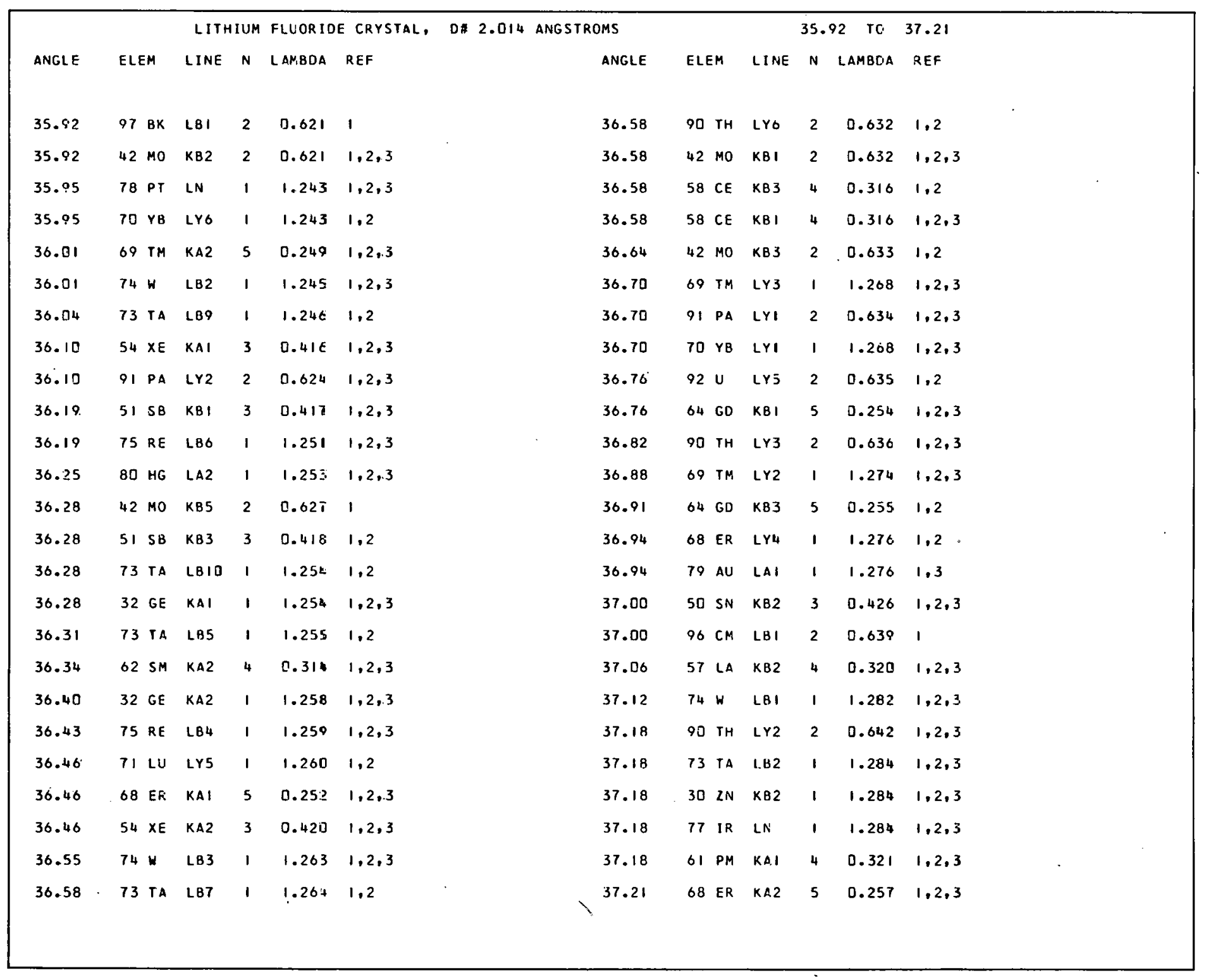




\begin{tabular}{|c|c|c|c|c|c|c|c|c|c|c|c|c|c|c|}
\hline & & LITH & IUM & FLUORID & E CRYSTAL, & $D \# 2.014$ & ANGSTROMS & & & & 37. & TO & 38.62 & \\
\hline ANGLE & ELEM & LINE & $\mathrm{N}$ & LAMBDA & REF & & ANGLE & ELE & & LINE & N & LAMBDA & REF & \\
\hline 37.21 & 63 EU & KB2 & 5 & 0.257 & $1,2,3$ & & 37.90 & 50 & SN & KB3 & 3 & 0.436 & 1.2 & \\
\hline 37.21 & $302 \mathrm{~N}$ & KB5 & 1 & 1.285 & 1,2 & & 37.96 & 91 & PA & L.Y5 & 2 & 0.655 & 1,2 & \\
\hline 37.24 & $44 \mathrm{RU}$ & KAI & 2 & 0.643 & $1,2,3$ & & 38.02 & 57 & LA & KBI & 4 & 0.328 & $1,2,3$ & \\
\hline 37.30 & $79 \mathrm{AU}$ & LA2 & 1 & 1.288 & $1,2,3$ & & 38.05 & 78 & PI & LAI & 1 & 1.313 & $1,2,3$ & \\
\hline 37.36 & $72 \mathrm{HF}$ & LB9 & 1 & 1.290 & 1,2 & . & 38.08 & 53 & 1 & KA2 & 3 & 0.438 & $1,2,3$ & \\
\hline 37.36 & $74 \mathrm{~W}$ & 286 & 1 & 1.290 & $1,2,3$ & & 38.11 & 68 & ER & LY3 & 1 & 1.315 & $1,2,3$ & \\
\hline 37.36 & 69 TM & LYG & 1 & 1.290 & 1 & & 38.11 & 69 & $T M$ & LY! & 1 & 1.315 & $1,2,3$ & \\
\hline 37.48 & $44 \mathrm{RU}$ & KA2 & 2 & 0.647 & $1,2,3$ & & 38.14 & 57 & LA & KB3 & 4 & 0.329 & 1,2 & \\
\hline 37.51 & $30 \mathrm{ZN}$ & KBI & 1 & 1.295 & $1,2,3$ & & 38.14 & 83 & $B I$ & LL & 1 & 1.316 & $1,2,3$ & \\
\hline 37.54 & $50 \mathrm{SN}$ & KB5 & 3 & 0.432 & 1 & & 38.14 & 95 & AM & LBI & 2 & 0.658 & 1,3 & \\
\hline 37.60 & $72 \mathrm{HF}$ & LBIJ & 1 & 1.298 & 1,2 & & 38.26 & 41 & NB & KBS & 2 & 0.660 & 1 & \\
\hline 37.60 & $72 \mathrm{HF}$ & LB5 & 1 & 1.298 & 1.2 & & 38.26 & 63 & EU & $k 83$ & 5 & 0.264 & 1.2 & \\
\hline 37.60 & $88 R A$ & LY4 & 2 & 0.649 & 1,2 & & 38.26 & 63 & EU & KB I & 5 & 0.264 & $1,2,3$ & \\
\hline 37.63 & .531 & KAI & 3 & 0.433 & $1,2,3$ & & 38.29 & 68 & ER & LY2 & 1 & 1.321 & $1,2,3$ & \\
\hline 37.66 & $61 \mathrm{PM}$ & KA2 & 4 & 0.325 & $1,2,3$ & & 38.32 & 67 & HO & $L Y_{4}$ & 1 & 1.322 & 1,2 & \\
\hline 37.72 & $74 \mathrm{~W}$ & L배 & 1 & 1.302 & $1,2,3$ & & 38.38 & 78 & PT & LA2 & 1 & 1.324 & $1,2,3$ & \\
\hline 37.78 & $57 \mathrm{LA}$ & K85 & 4 & 0.326 & 1 & & 38.44 & 72 & HF & LB2 & 1 & 1.326 & $1,2,3$ & \\
\hline 37.81 & $67 \mathrm{HO}$ & KAI & 5 & 0.261 & $1,2,3$ & & 38.47 & 73 & TA & LBI & 1 & 1.327 & $1,2,3$ & \\
\hline 37.81 & 50 sid & $K B I$ & 3 & 0.435 & $1,2,3$ & & 38.50 & 76 & OS & LN & 1 & 1.328 & $1,2,3$ & \\
\hline 37.84 & $70 \mathrm{YB}$ & LYS & 1 & 1.306 & 1.2 & & 38.50 & 60 & ND & KAI & 4 & 0.332 & $1,2,3$ & \\
\hline 37.84 & $90 \mathrm{TH}$ & LYI & 2 & 0.653 & $1,2,3$ & & 38.56 & 62 & SM & KB2 & 5 & 0.266 & $1,2,3$ & \\
\hline 37.84 & $98 \mathrm{CF}$ & LB2 & 2 & {$[\because .653$} & 1 & & 38.56 & 67 & HO & KA2 & 5 & 0.266 & $1,2,3$ & \\
\hline 37.84 & $72 \mathrm{HF}$ & L87 & 1 & 1.326 & 1,2 & & 38.59 & 73 & TA & LB6 & 1 & 1.331 & $1,2,3$ & \\
\hline 37.87 & 73 TA & LB3 & 1 & 1.307 & $1,2,3$ & & 38.62 & 56 & BA & $\mathrm{KB2}$ & 4 & 0.333 & $1,2,3$ & \\
\hline 37.91 & $41 N B$ & $K B 2$ & 2 & 0.654 & $1,2,3$ & & 38.62 & 41 & NB & KB3 & 2 & 0.666 & 1,2 & \\
\hline
\end{tabular}




\begin{tabular}{|c|c|c|c|c|c|c|c|c|c|c|c|c|c|}
\hline \multirow[b]{2}{*}{ ANGLE } & \multirow{2}{*}{ ELEM } & \multicolumn{2}{|c|}{ LITHIUM } & \multirow{2}{*}{\multicolumn{2}{|c|}{$\begin{array}{l}\text { FLUORIDE CRYSTAL, } \\
\text { LAMBOA REF }\end{array}$}} & \multirow{2}{*}{ D\# 2.014} & \multirow{2}{*}{$\begin{array}{l}\text { ANGSTROMS } \\
\text { ANGLE }\end{array}$} & \multicolumn{5}{|c|}{38.62 TO } & \multirow{2}{*}{$\begin{array}{l}40.07 \\
\text { REF }\end{array}$} \\
\hline & & LINE & $N$ & & & & & ELEM & & LINE & $N$ & LAMBDA & \\
\hline 38.62 & $41 \mathrm{NB}$ & KB I & 2 & 0.665 & $1,2,3$ & & 39.34 & $94 p$ & PU & LEI & 2 & 0.678 & 1 \\
\hline 38.71 & 49 IN & $K B 2$ & 3 & 0.445 & $1,2,3$ & & 39.53 & 771 & I R & $L 42$ & 1 & 1.362 & $1,2,3$ \\
\hline 38.74 & $71 \mathrm{LU}$ & LB9 & 1 & 1.335 & 1,2 & & 39.53 & $92 \mathrm{U}$ & u & LB9 & 2 & 0.681 & 1.2 \\
\hline 38.80 & $97 \mathrm{BR}$ & LB2 & 2 & ᄃ. 667 & 1 & $\cdot$ & 39.59 & $08 \mathrm{E}$ & ER & $L Y \mid$ & 1 & 1.364 & $1,2,3$ \\
\hline 38.86 & $31 \mathrm{GA}$ & KAI & 1 & $1.34 \mathrm{~J}$ & $1,2,3$ & & 39.59 & 568 & $B A$ & KBI & 4 & 0.341 & $1,2,3$ \\
\hline 38.92 & $71 \mathrm{LU}$ & LBS & 1 & 1.342 & 1,2 & & 39.59 & $67 \mathrm{H}$ & HO & LY3 & 1 & 1.364 & 1.2 .3 \\
\hline 38.95 & $71 L U$ & LBIS & 1 & 1.343 & 1,2 & & 39.59 & $56 \mathrm{~B}$ & $B A$ & $\mathrm{~KB} 3$ & 4 & 0.341 & 1,2 \\
\hline 38.98 & 60 ND & KA 2 & 4 & 0.336 & $1,2,3$ & & 39.59 & $88 R$ & RA & LYZ & 2 & 0.682 & $1,2,3$ \\
\hline 38.98 & $31 \mathrm{GA}$ & KA2 & 1 & 1.344 & $1,2, .3$ & & 39.62 & $49 \mathrm{I}$ & IN & KB3 & 3 & 0.455 & 1,2 \\
\hline 39.04 & $73 \mathrm{TA}$ & $\mathrm{LB}_{4}$ & 1 & 1.346 & $1,2,3$ & & 39.62 & $62 \mathrm{~s}$ & $S M$ & KBI & 5 & 0.273 & $1,2,3$ \\
\hline 39.04 & $83 \mathrm{RA}$ & Lro & 2 & 0.673 & 1,2 & & 39.62 & 491 & IN & KBI & 3 & 0.455 & $1,2,3$ \\
\hline 39.04 & $43 \mathrm{rC}$ & KAI & 2 & 0.673 & 2,3 & & 39.71 & $52 T$ & TE & KA2 & 3 & 0.456 & $1,2,3$ \\
\hline 39.10 & 87 AC & LYI & 2 & 0.674 & 1 & & 39.77 & $67 \mathrm{H}$ & HO & $\operatorname{Lr} 2$ & 1 & 1.370 & $1,2,3$ \\
\hline 39.13 & ניا וד & LB? & 1 & 1.349 & 1,2 & & 39.77 & $66 \mathrm{D}$ & DY & KA2 & 5 & 0.274 & $1,2,3$ \\
\hline 39.16 & $82 \mathrm{~PB}$ & $\mathrm{LL}$ & 1 & $1.3 \leq 0$ & $1,2,3$ & & 39.77 & $62 \mathrm{~s}$ & SM & K33 & 5 & 0.274 & 1,2 \\
\hline $39.1 t$ & $90 \mathrm{TH}$ & LYS & 2 & 0.675 & 1,2 & & 39.77 & $96 \mathrm{C}$ & $C M$ & L32 & 2. & 0.685 & 1 \\
\hline $39.1 \epsilon$ & $88 R A$ & $\operatorname{LY} 3$ & 2 & 0.675 & $1,2,3$ & & 39.77 & 712 & LU & 282 & 1 & 1.370 & $1,2,3$ \\
\hline 39.16 & o6 or & KAI & 5 & 0.270 & $1,2,3$ & & 39.86 & $75 R$ & RE & LN & 1 & 1.373 & $1,2,3$ \\
\hline 39.18 & $77 \mathrm{IR}$ & $\lfloor A \mid$ & 1 & 1.351 & $1,2,3$ & & 39.89 & 660 & DY & $\mathrm{LY}_{4}$ & 1 & 1.374 & 1,2 \\
\hline 39.22 & $43 \mathrm{TC}$ & $K A 2$ & 2 & 0.676 & 2,3 & & 39.89 & $72 \mathrm{H}$ & HF & LB6 & 1 & 1.374 & $1,2,3$ \\
\hline 39.22 & $56 \mathrm{BA}$ & KB5 & 4 & 0.358 & 1 & & 39.89 & $72 \mathrm{H}$ & $\mathrm{HF}$ & $|B|$ & 1 & 1.374 & $1,2,3$ \\
\hline 39.25 & $49 \mathrm{IN}$ & K85 & 3 & 3.451 & 1 & & 39.89 & $92 \mathrm{U}$ & $\mathbf{u}$ & LBIO & 2 & 0.687 & 1,2 \\
\hline 39.25 & $72 \mathrm{HF}$ & $\angle B 3$ & 1 & 1.353 & $1,2,3$ & & 39.95 & $59 p$ & $P R$ & KAI & 4 & 0.344 & $1,2,3$ \\
\hline 39.25 & $52 \mathrm{TE}$ & KAL & 3 & 0.451 & $1,2,3$ & & 40.07 & $61 P$ & PM & KB2 & 5 & 0.276 & 1. \\
\hline 39.37 & $69 \mathrm{TM}$ & LYS & 1 & 1.355 & 1,2 & & 40.07 & 402 & $2 R$ & KB2 & 2 & 0.690 & $1,2,3$ \\
\hline
\end{tabular}




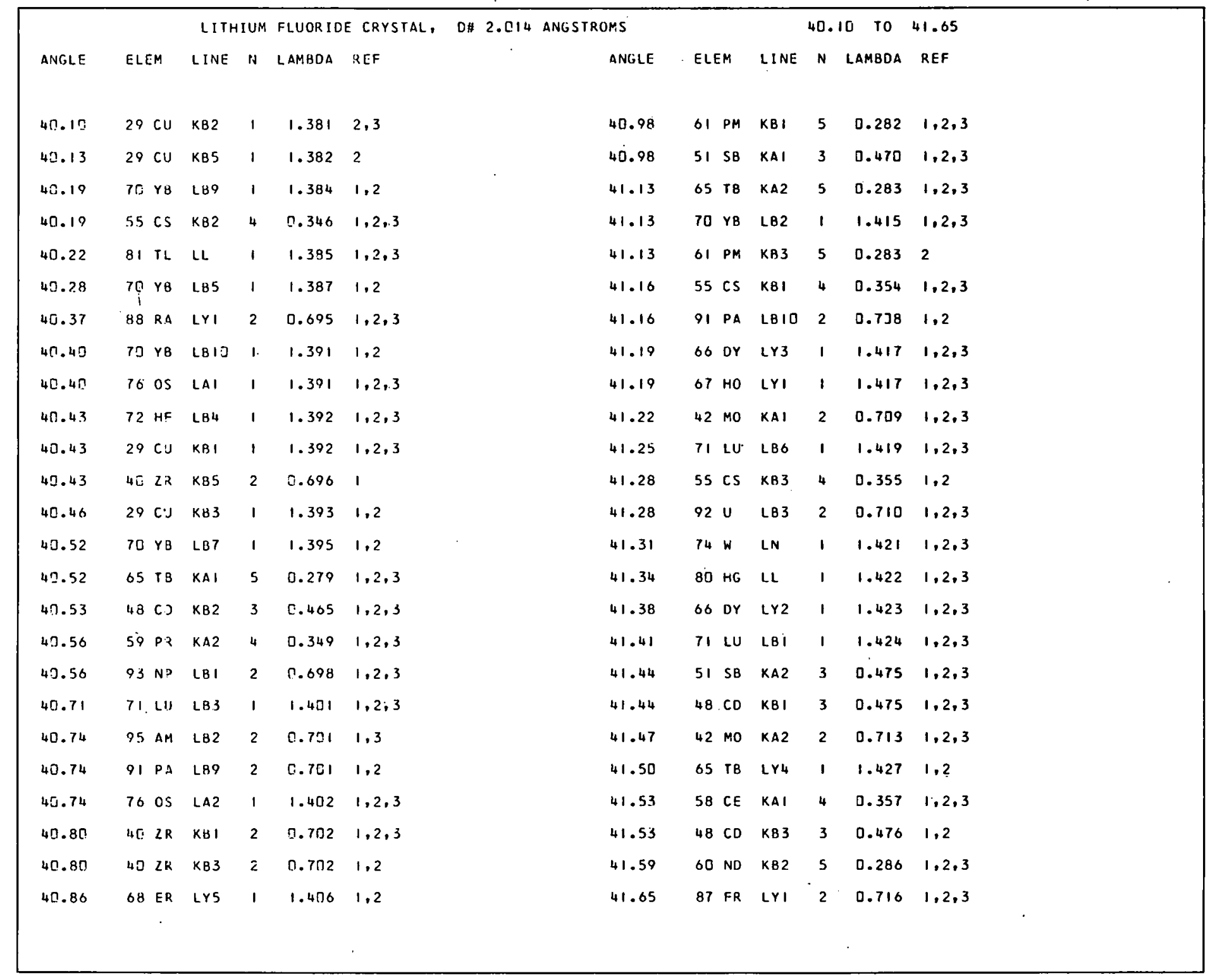




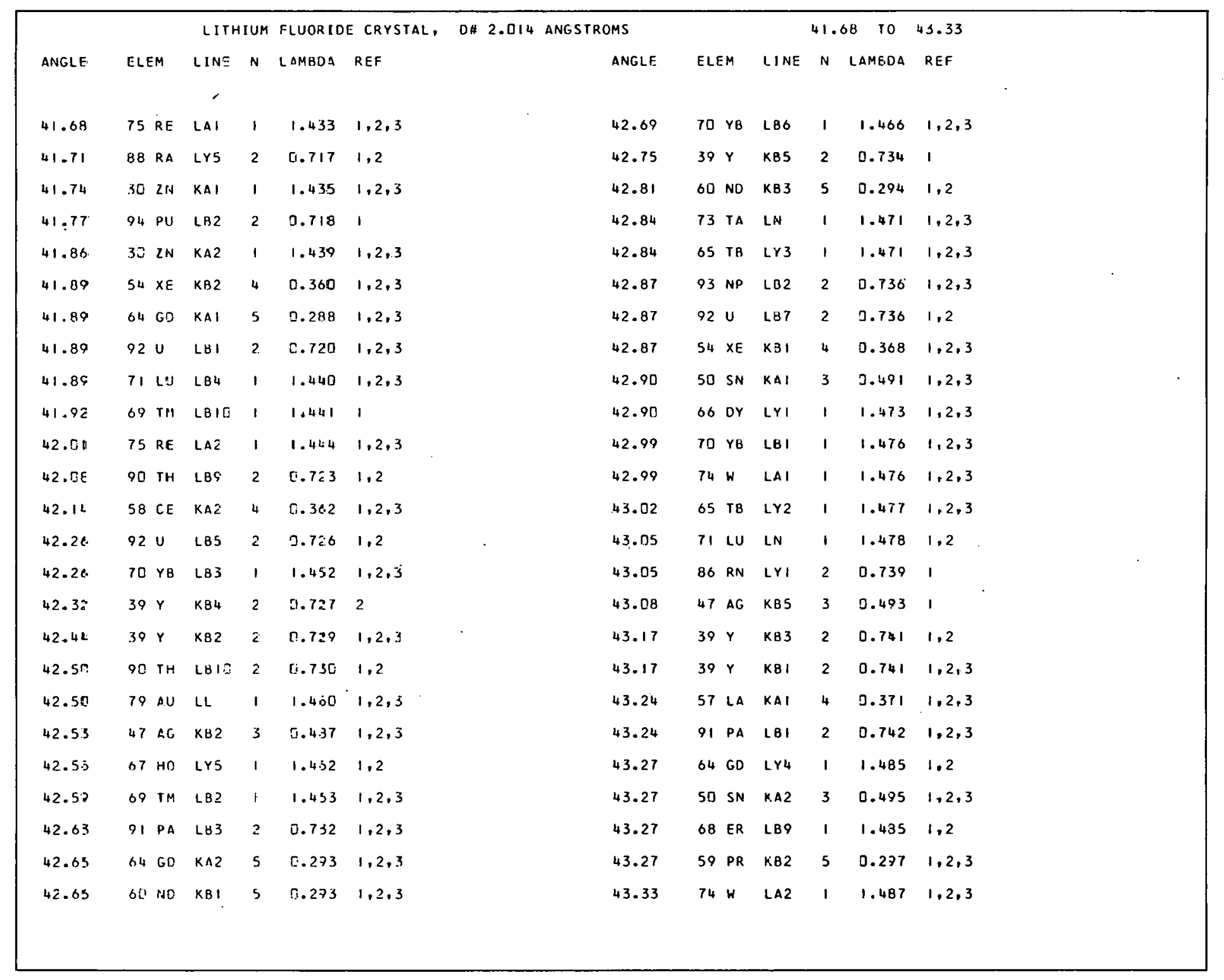




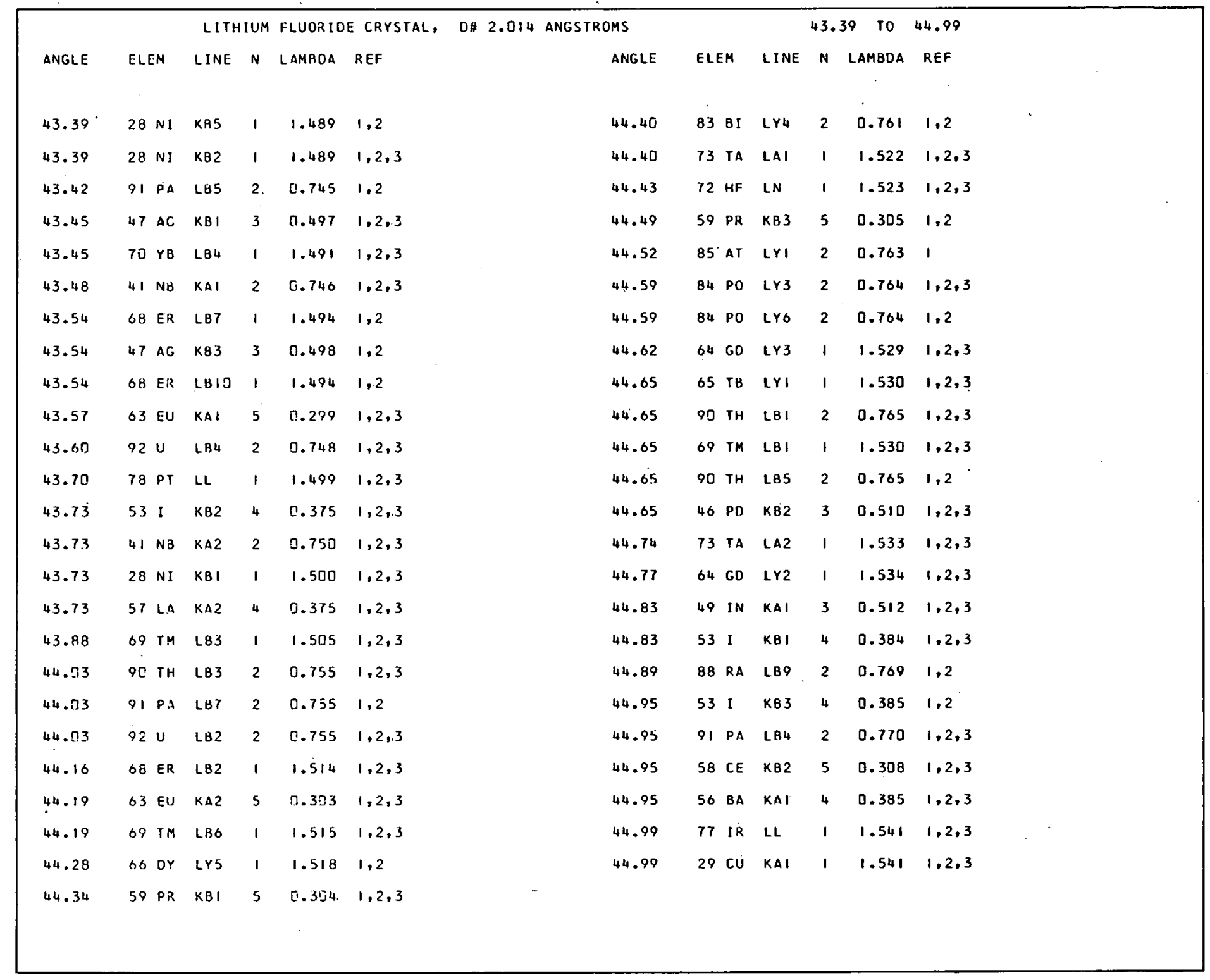




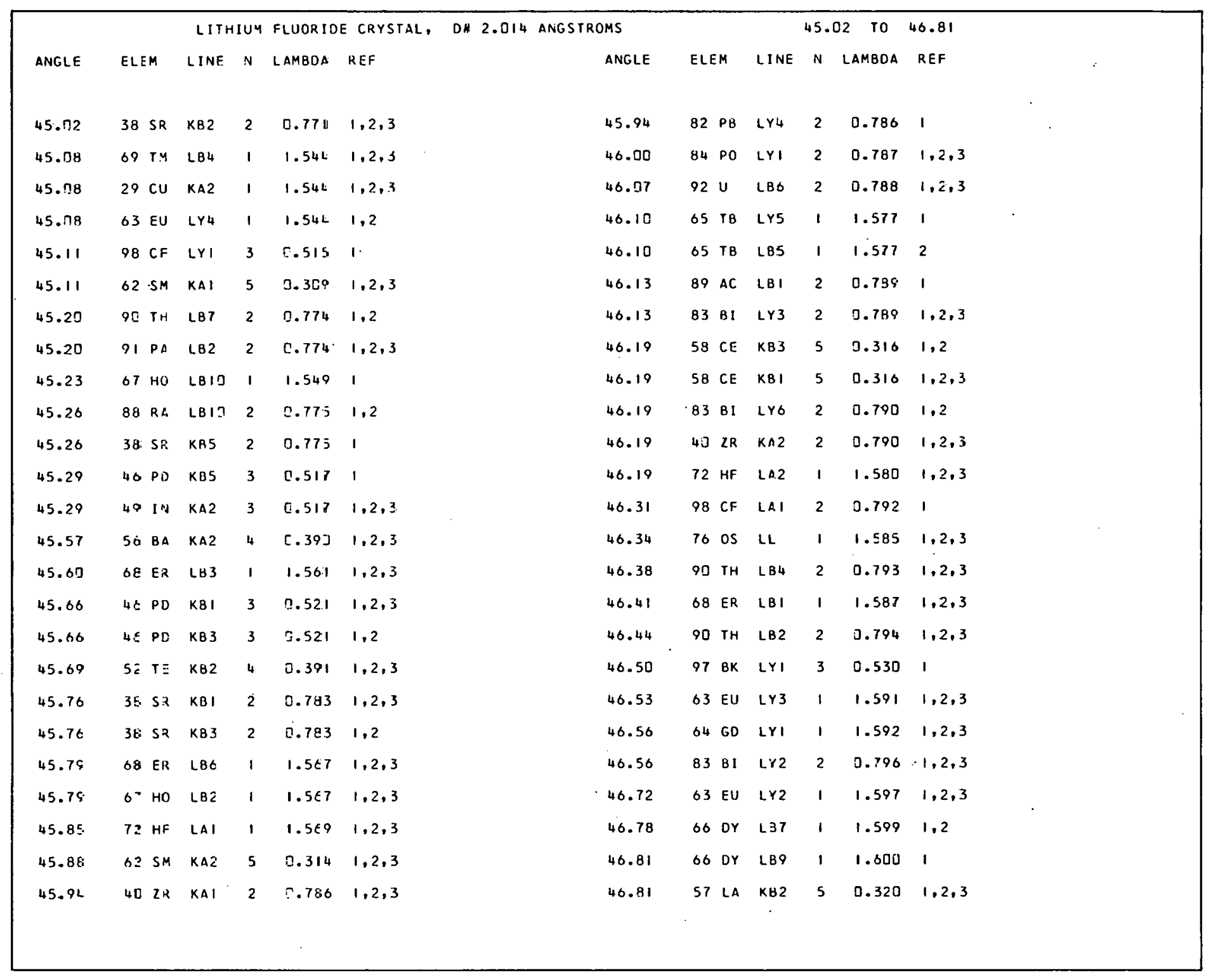




\begin{tabular}{|c|c|c|c|c|c|c|c|c|c|c|c|c|c|c|}
\hline$\cdot$ & & LITH & IIUM & FLUORID & E CRYSTAL, & D\# 2.014 & ANGSTROMS & & & & 46. & To & 48.55 & \\
\hline ANGLE & ELEM & LINE & N & LAMBDA & REF & & ANGLE & ELE & & LINE & N & LAMBDA & REF & \\
\hline 46.81 & $55 \mathrm{cs}$ & $K A \mid$ & 4 & 0.400 & $1,2,3$ & & 47.58 & 61 & PM & KA2 & 5 & 0.325 & $1,2,3$ & \\
\hline 46.81 & 52 I & $\mathrm{KBI} \mathrm{I}^{\circ}$ & 4 & 0. & $1,2,3$ & . & 47.62 & 83 & 81 & $L Y \mid$ & 2 & 0.813 & $1,2,3$ & \\
\hline 46.84 & $.68 \mathrm{ER}$ & LB4 & 1 & 1.601 & $1,2,3$ & & 47.68 & 89 & $A C$ & LB2 & 2 & 0.814 & 1 & \\
\hline 46.93 & $52 \mathrm{TE}$ & $K B 3$ & 4 & 0.401 & 1,2 & & 47.68 & 88 & RA & LBI & 2 & 0.814 & $1,2,3$ & \\
\hline 46.96. & $48 \mathrm{CD}$ & KAI & 3 & 0.535 & $1,2,3$ & & 47.74 & 71 & LU & LA2 & 1 & 1.630 & $1,2,3$ & \\
\hline 46.96 & $61 \mathrm{PM}$ & KAI & 5 & 0.321 & $1,2,3$ & & 47.74 & 82 & PH & LY3 & 2 & 0.815 & $1,2,3$ & \\
\hline 46.96 & $45 \mathrm{RH}$ & KB2 & 3 & 0.535 & $1,2,3$ & & 47.74 & 75 & RE & LL & 1 & 1.630 & $1,2,3$ & \\
\hline 46.99 & $62 \mathrm{SM} . \mathrm{H}$ & LY4 & 1 & 1.606 & 1,2 & & 47.711 & 57 & LA & KBS & 5 & 0.326 & 1 & \\
\hline 46.99 & $88 \mathrm{RA}$ & LB3 & 2 & 0.80 .3 & $1,2,3$ & . & 47.80 & 88 & RA & LB7 & 2 & 0.816 & 1,2 & \\
\hline 47.03 & 66 OY & LBIJ & 1 & 1.607 & 1 & & 47.80 & 51 & SB & KB2 & 4 & 0.408 & $1,2.3$ & \\
\hline 47.56 & $98 \mathrm{CF}$ & LA2 & 2 & 0.804 & 1 & & 47.80 & 37 & RB & KB2 & 2 & 0.816 & $1,2,3$ & \\
\hline 47.09 & $27 \mathrm{CO}$ & KB5 & 1 & 1.609 & 1,2 & & 47.86 & 82 & PB & LYG & 2 & 0.817 & 1,2 & \\
\hline 47.12 & 920 & LN & 2 & 0.805 & $1,2,3$ & & 47.93 & 70 & $Y B$ & LN & 1 & 1.636 & $1,2,3$ & \\
\hline 47.18. & $88 R A$ & LB5 & 2 & 0.806 & 1,2 & & 47.99 & 45 & $\mathrm{RH}$ & $K B 3$ & 3 & 0.546 & 1,2 & \\
\hline 47.30 & $91 \mathrm{PA}$ & LB6 & 2 & 0.828 & $1,2,3$ & & 47.99 & 96 & $C M$ & $|Y|$ & 3 & 0.546 & 1 & \\
\hline 47.34 & $48 C D$ & KA2 & 3 & $\pi .539$ & $1,2,3$ & & 47.99 & 45 & $\mathrm{RH}$ & KBI & 3 & 0.546 & $1,2,3$ & \\
\hline 47.40 & $71 \mathrm{LIJ}$ & LAL & 1 & 1.619 & $1,2,3$ & & 48.05 & 57 & LA & KBI & 5 & 0.328 & $1,2,3$ & \\
\hline 47.40 & $67 \mathrm{HO}$ & LB 3 & 1 & 1.619 & $1,2,3$ & & 48.08 & 64 & GD & LYS & 1 & 1.641 & 1 & \\
\hline 47.43 & $55 \mathrm{cs}$ & KA2 & 4 & 0.405 & $1,2,3$ & & 48.11 & 82 & PB & LY2 & 2 & 0.821 & $1,2,3$ & \\
\hline 47.43 & $97 \mathrm{bK}$ & LAI & 2 & 0.810 & 1 & & 48.18 & 37 & RB & K85 & 2 & 0.822 & 1 & \\
\hline 47.46 & $27 \mathrm{CO}$ & $K B_{1}$ & 1 & 1.621 & $1,2,3$ & & 48.18 & 97 & $B K$ & LA2 & 2 & 0.822 & 1 & \\
\hline 47.49 & $67 \mathrm{HO}$ & LB6 & 1 & 1.622 & $1,2,3$ & & 48.21 & 57 & LA & K.B 3 & 5 & 0.329 & 1,2 & . \\
\hline 47.52 & $66 \mathrm{DY}$ & LB2 & 1 & 1.623 & $1,2,3$ & & 48.27 & 67 & HO & LB I & 1 & 1.647 & $1,2,3$ & \\
\hline 47.52 & $45 \mathrm{RH}$ & KB5 & 3 & 0.541 & 1 & & 48.52 & 62 & SM & LY3 & 1 & 1.655 & $1,2,3$ & \\
\hline 47.55 & $81 \mathrm{TL}$ & Lru & 2 & 0.812 & 1,2 & & 48.55 & 51 & SB & KBS & 4 & 0.414 & 1 & \\
\hline
\end{tabular}




\begin{tabular}{|c|c|c|c|c|c|c|c|c|c|c|c|c|c|c|c|}
\hline & & LITH & IUM & FLUOR ID & E CRYSTAL, & $D \# 2.014$ & ANGSTROMS & & & & 48. & $55 \mathrm{ra}$ & 53.18 & & \\
\hline ANGLE & ELEM & LINE & $N$ & $\angle A M B D A$ & REF & & ANGLE & ELE & & LINE & N & LAMBDA & REF & & \\
\hline 48.55 & $9 \mathrm{C} \mathrm{TH}$ & LBO & 2 & $0.82 \varepsilon$ & $1,2,3$ & & 49.30 & 82 & $P B$ & $L Y \mid$ & 2 & 0.840 & $1,2,3$ & & \\
\hline 48.61 & $91 P A$ & $L N$ & 2 & 0.829 & $1,2,3$ & . & 49.30 & 87 & $F R$ & LBI & 2 & 0.840 & $1,2,3$ & & \\
\hline 48.01 & $37 \mathrm{RB}$ & XB3 & 2 & 0.826 & 1,2 & & 49.30 & 60 & No & $K A 2$ & 5 & 0.336 & $1,2,3$ & , & . \\
\hline 48.51 & $37 \mathrm{RA}$ & $K B 1$ & 2 & $0.82 \%$ & $1,2,3$ & & 49.30 & 54 & $X E$ & $K A 2$ & 4 & 0.420 & $1,2,3$ & & \\
\hline 48.51 & $96 \mathrm{CM}$ & LA I & 2 & 0.829 & 1 & & 49.33 & 66 & OY & LBS & 1 & 1.681 & $1,2,3$ & & \\
\hline 48.51 & $39 Y$ & KAI & 2 & 0.825 & $1,2,3$ & & 49.33 & 66 & DY & $L 83$ & 1 & 1.681 & $1,2,3$ & & \\
\hline 48.31 & $63 \mathrm{EU}$ & LYI & 1 & 1.658 & $1,2,3$ & & 49.36 & 96 & $C M$ & LA2 & 2 & 0.841 & 1 & & \\
\hline 48.61 & $28 \mathrm{NI}$ & KAI & 1 & 1.658 & $1,2,3$ & & 49.36 & 65 & TB & L82 & 1 & 1.632 & $1,2,3$ & & \\
\hline 48.64 & $67 \mathrm{HO}$ & $\mathrm{LBH}_{4}$ & 1 & $1.65^{\circ}$ & $1,2,3$ & & 49.36 & 81 & TL & $\operatorname{LY} 3$ & 2 & 0.841 & $1,2,3$ & & \\
\hline 48.64 & $65 T 8$ & LB7 & 1 & $1.65^{\circ}$ & 1,2 & & 49.36 & 88 & RA & L84 & 2 & 0.841 & $1,2,3$ & & \\
\hline 48.64 & $62 \mathrm{SN}$. & LYZ & 1 & $1.65^{\circ}$ & $1,2,3$ & & 49.39 & 79 & YE & LA2 & 1 & 1.083 & $1,2,3$ & & \\
\hline 48.68 & 6.: NO & KAI & 5 & 0.332 & $1,2,3$ & & 49.49 & 95 & $A M$ & LYI & 3 & 0.562 & 1,3 & & \\
\hline 48.74 & $28 \mathrm{NI}$ & $K A 2$ & 1 & 1.662 & $1,2,3$ & & 49.49 & 44 & $\mathrm{RU}$ & $\mathrm{KB} 2$ & 3 & 0.562 & $1,2,3$ & & \\
\hline 48.80 & $54 \times E$ & KAI & 4 & 0.41 .5 & $1,2,3$ & & 49.55 & 81 & $\mathrm{TL}$ & LYO & 2 & 0.844 & 1,2 & & \\
\hline 48.83 & $56 \mathrm{BA}$ & KB2 & 5 & $\therefore .333$ & $1,2,3$ & & 49.61 & 56 & $8 A^{-}$ & Kes & 5 & 0.338 & 1 & & \\
\hline 48.86 & $39 \mathrm{Y}$ & KA2 & 2 & 0.833 & $1,2,3$ & & 49.68 & 47 & $A G$ & $K A .2$ & 3 & 0.564 & $1,2,3$ & & 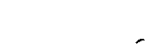 \\
\hline 48.89 & $6 S T B$ & LOIE & 1 & 1.067 & 1,2 & & 49.80 & 81 & $\mathrm{TL}$ & LY2 & 2 & 0.848 & $1,2,3$ & & \\
\hline 48.92 & SI SB & Кв I & 4 & 0.417 & $1,2,3$ & & 49.85 & 95 & $A M$ & $(A)$ & 2 & 0.848 & 1,3 & & $\cdot$ \\
\hline 48.99 & $B E R A$ & LB2 & 2 & 0.835 & $1,2,3$ & & 49.8[ & 69 & $T M$ & LN & 1 & 1.696 & $1,2,3$ & & \\
\hline 49.05 & $51 S B$ & KB3 & 4 & 0.418 & 1,2 & & 50.05 & 44 & $R U$ & KAS & 3 & 0.563 & 1 & & \\
\hline 49.05 & $7\left[Y_{B}\right.$ & $(A)$ & 1 & 1.672 & $1,2,3$ & & 50.05 & 50 & $S N$ & $K B 2$ & 4 & 0.423 & $1,2,3$ & & \\
\hline 49.21 & 4? $A G$ & KAI & 3 & 0.559 & $1,2,3$ & & 50.08 & 56 & $B A$ & $K B I$ & 5 & 0.341 & $1,2,3$ & & \\
\hline 49.24 & $80 \mathrm{HG}$ & LY4 & 2 & 0.8 .59 & 1,2 & & 50.28 & 56 & $B A$ & $\mathrm{~KB} 3$ & 5 & 0.341 & 1,2 & & \\
\hline 49.24 & $7 \mathrm{LW}$ & LL & 1 & 1.678 & $1,2,3$ & & 50.18 & 90 & $\mathrm{TH}$ & LN & 2 & 0.854 & $1,2,3$ & & \\
\hline 49.24 & 8381 & LYS & 2 & 0.839 & 1,2 & - & 50.18 & 63 & EU & LYS & 1. & 1.708 & 1,2 & & \\
\hline
\end{tabular}




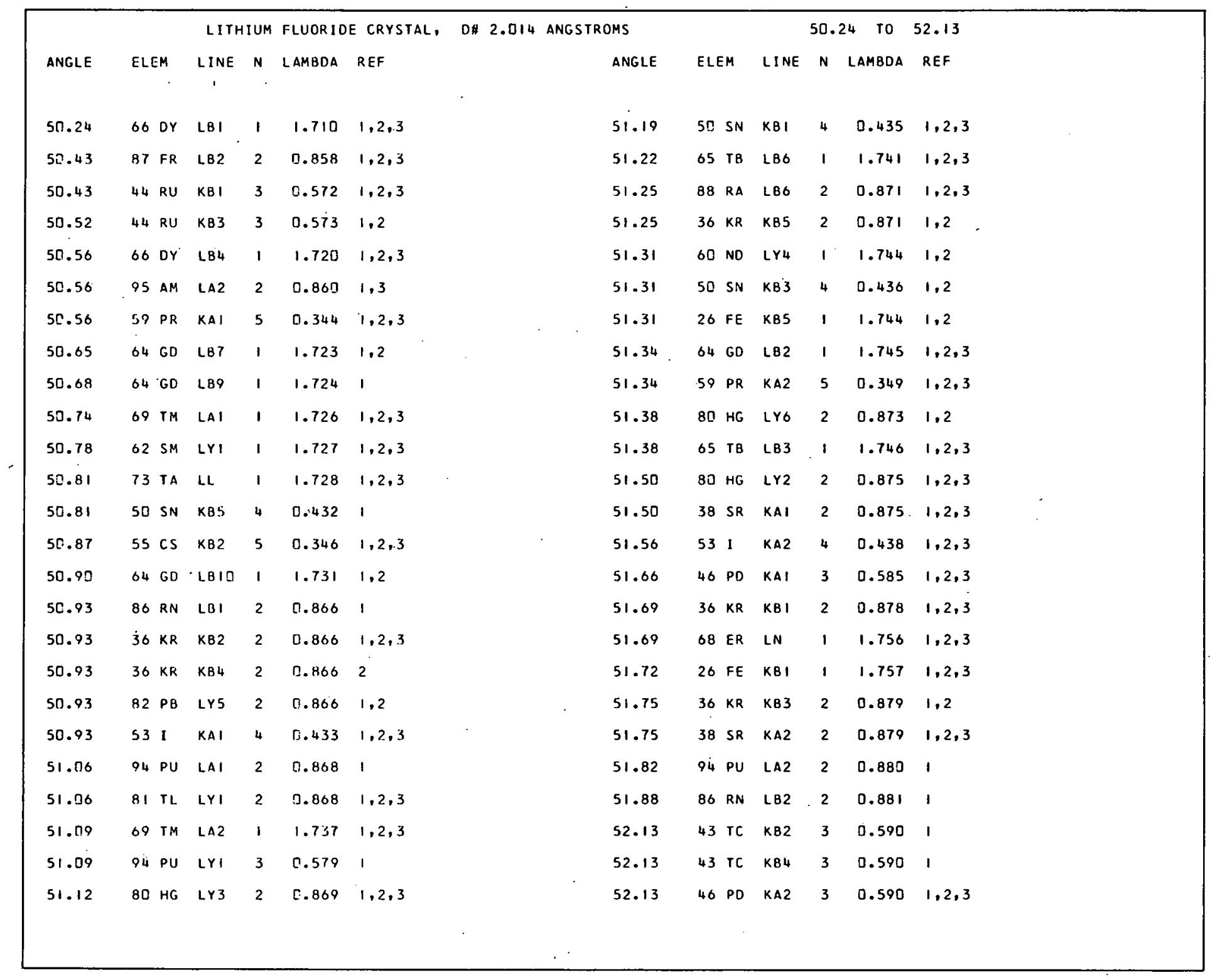




\begin{tabular}{|c|c|c|c|c|c|c|c|c|c|c|c|c|c|c|c|c|}
\hline & & LITH & IUN & FLUOR:D & E CRYSTAL, & DH 2 & 2.014 & ANGSTROMS & & & & 52. & 13 T0 & 53.69 & & \\
\hline ANCLE & F:LEM & LINE & $N$ & LAMBDA & REF & & & ANGLE & ELE & EM & LINE & $\mathbf{N}$ & LAMBDA & REF & & 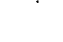 \\
\hline 52.13 & $55 \mathrm{Cs}$ & KBI & 5 & c.35t & $1,2,3$ & & & 52.96 & 83 & BI & LE. & 2 & 0.898 & 1,2 & & \\
\hline 52.29 & $5.5 \mathrm{cs}$ & KB.3 & 5 & 5.355 & 1,2 & & & 52.96 & 60 & No & LY3 & 1 & 1.796 & $1,2,3$ & & \\
\hline 52.32 & $65 \mathrm{~TB}$ & LBI & 1 & 1.775 & $1,2,3$ & & & 53.52 & 84 & PO & LB5 & 2 & 0.899 & 1.2 & - & \\
\hline 52.39 & $93 \mathrm{NH}$ & LAI & 2 & $\because .887$ & $1,2,3$ & & & 53.09 & 54 & $X E$ & $K B 2$ & 5 & $0.361]$ & $1,2,3$ & & \\
\hline 52.42 & $625 \mathrm{si}$ & LB5 & 1 & 1.779 & 2 & & & 53.09 & 63 & EU & LBID & 1 & 1.800 & 1.2 & & \\
\hline $52.1,2$ & $62 \mathrm{SH}$ & LY5 & 1 & 1.779 & 1 & . & & 53.12 & 60 & NO & LY2 & 1 & 1.801 & $1,2,3$ & & \\
\hline 52.45 & $49 ! 1:$ & $\mathrm{K} 82$ & 4 & 5.445 & $1,2,3$ & & & 53.15 & 93 & NP & LA2 & 2 & 0.901 & 1 & & \\
\hline 52.48 & $72 \mathrm{HF}$ & $\mathrm{Li}$ & 1 & 1.781 & $1,2,3$ & & & 53.18 & 43 & $T C$ & $\mathrm{~KB} 3$ & 3 & 0.601 & 1,2 & & \\
\hline 52.51 & $91 \mathrm{PA}$ & LY4 & 3 & 0.594 & 1,2 & & & 53.21 & 49 & IN & KBS & 4 & 0.451 & 1 & & \\
\hline $52.5 \varepsilon$ & 66 ER & LAI & 1 & 1.784 & $1,2,3$ & & & 53.21 & 52 & $T E$ & $K \cap 1$ & 4 & 0.451 & $1,2,3$ & & \\
\hline 52.61 & $65 \mathrm{~TB}$ & LBH & 1 & 1.785 & $1,2,3$ & & & 53.28 & 79 & $A U$ & LYo & 2 & 0.903 & 1,2 & & \\
\hline 52.61 & $92 \mathrm{U}$ & LYG & 3 & 0.555 & 1,2 & & & 53.28 & 43 & IC & KBI & 3 & 0.302 & $1,2,3$ & & \\
\hline 52.61 & $58 \mathrm{CE}$ & KA! & 5 & 0.35 .7 & $1,2,3$ & & & 53.31 & 64 & GD & LB 6 & 1 & 1.307 & $1,2,3$ & & \\
\hline 52.70 & OS EI & LBT & 1 & 1.788 & 1,2 & & & 53.34 & 79 & $A U$ & LY2 & 2 & 0.704 & $1,2,3$ & & \\
\hline 52.70 & $B 5 \mathrm{AT}$ & LEI & 2 & $5.8 a^{\circ}$ & 1 & & & 53.37 & 98 & $\mathrm{CF}$ & LBI & 3 & 0.603 & 1 & & \\
\hline 52.74 & $27 \mathrm{CO}$ & KAI & 1 & 1.789 & $1,2,3$ & & & $53.4 \mathrm{C}$ & 83 & $B I$ & LBIO & 2 & 0.905 & 1,2 & & \\
\hline $52.7^{7}$ & $B I \mathrm{TL}$ & LYS & 2 & 0.805 & 1,2 & & & 53.40 & 58 & $C E$ & KA2 & 5 & 0.362 & $1,2,3$ & & \\
\hline 52.83 & $93 \mathrm{~N} 9$ & $|Y|$ & 3 & 0.507 & $1,2,3$ & & & 53.40 & 85 & $A T$ & LB2 & 2 & 0.905 & 1 & & \\
\hline 52.83 & $63 \mathrm{EU}$ & LBQ & 1 & 1.7 .02 & 1,2 & & & 53.47 & 63 & EU & LB2 & 1 & 1.812 & $1,2,3$ & & \\
\hline 52.83 & $27 \mathrm{co}$ & $K \Lambda \geq$ & 1 & 1.773 & $1,2,3$ & & & 53.53 & 88 & RA & LN & 2 & 0.907 & $1,2,3$ & & \\
\hline 52.910 & $78 \mathrm{PT}$ & $\mathrm{LY}_{4}$ & 2 & 0.877 & 1,2 & & & 53.56 & 92 & $u$ & LY2 & 3 & 0.605 & $1,2,3$ & & \\
\hline 52.92 & 8. HG & $|r|$ & 2 & 0.877 & $1,2,3$ & & $:$ & 53.56 & 64 & GD & LB3 & 1 & 1.815 & $1,2,3$ & & \\
\hline 52.93 & $92 \mathrm{U}$ & LY3 & 3 & 5.578 & $1,2,3$ & & & 53.60 & 61 & $P M$ & $|Y|$ & 1 & 1.816 & 1 & & . \\
\hline 52.96 & OH ER & LA2 & 1 & 1.796 & $-1,2,3$ & & & 53.66 & 84 & PO & LB3 & 2 & 0.9 .39 & $1,2,3$ & & \\
\hline 52.76 & 79 All & LY3 & 2 & $\because 898$ & $1,2,3$ & & & 53.69 & 59 & $\mathrm{PR}$ & LYu & 1 & 1.819 & 1.2 & . & \\
\hline
\end{tabular}




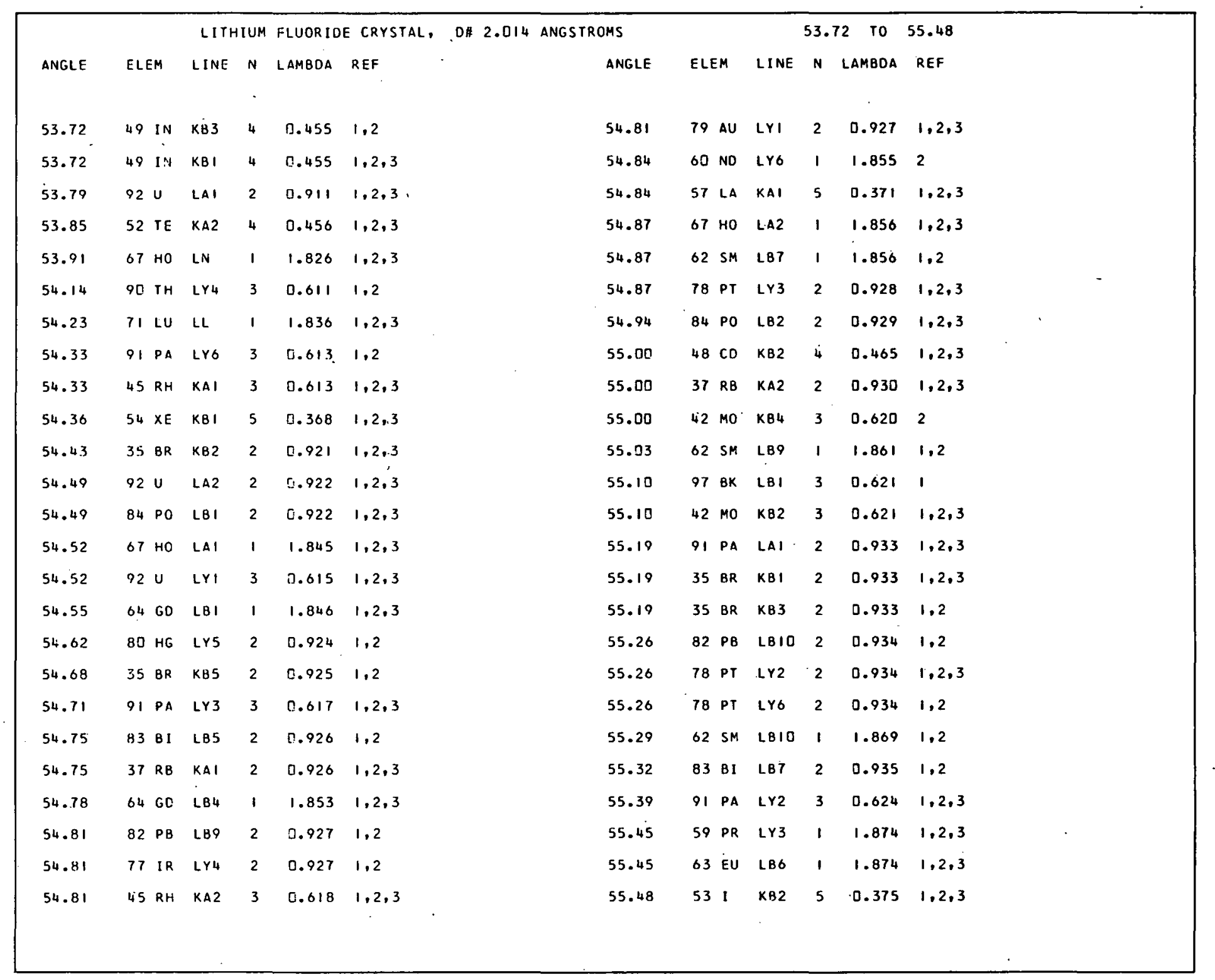




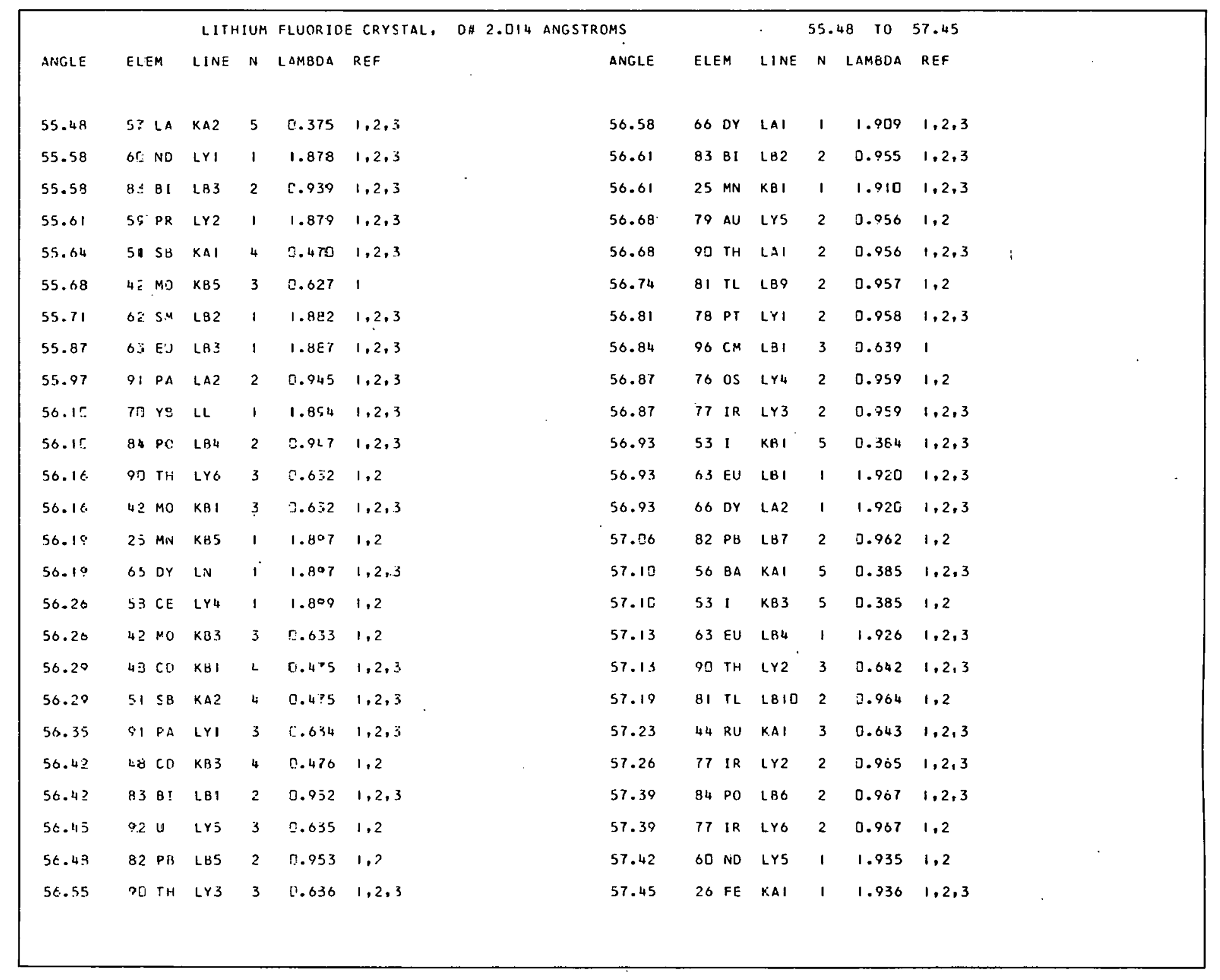




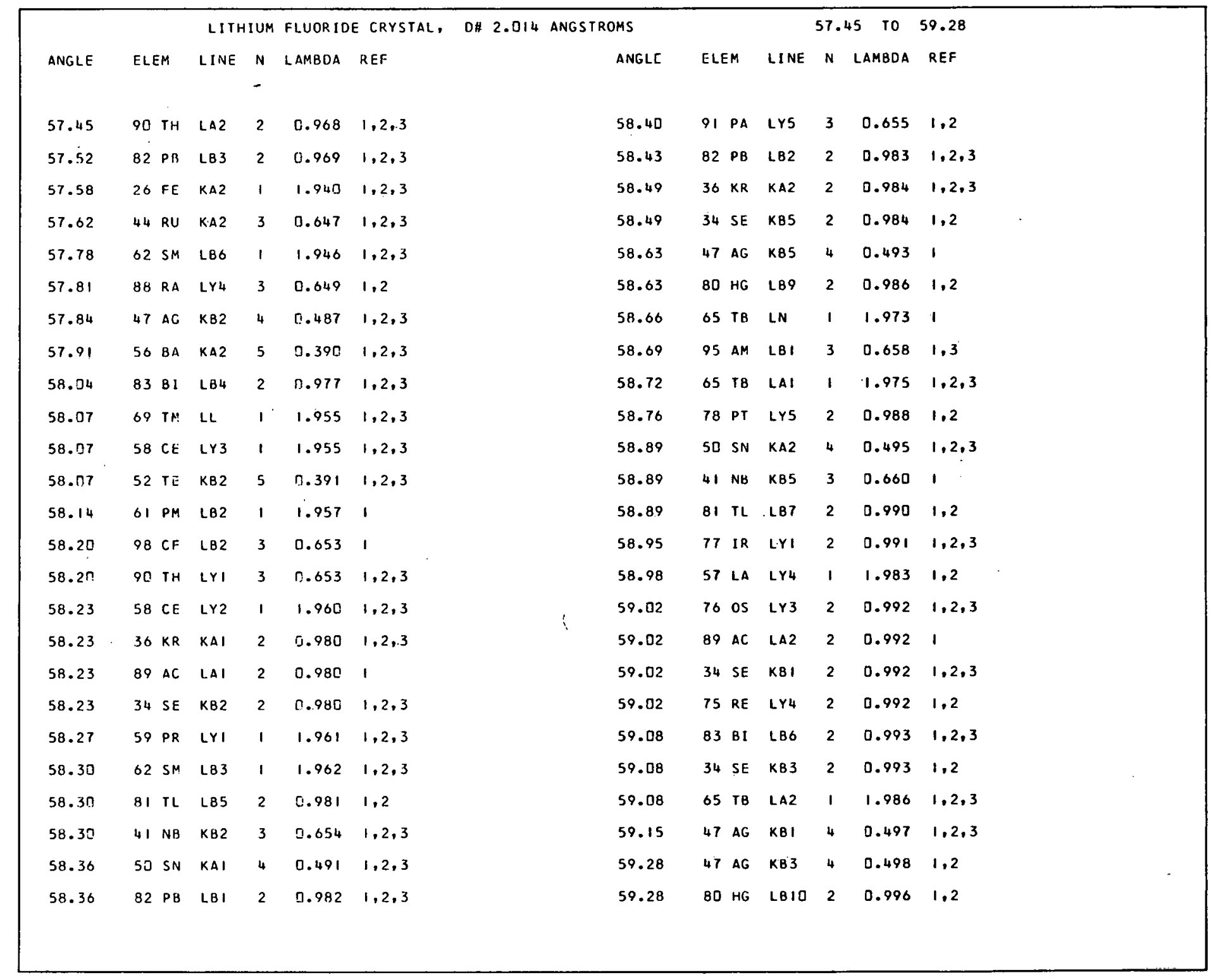




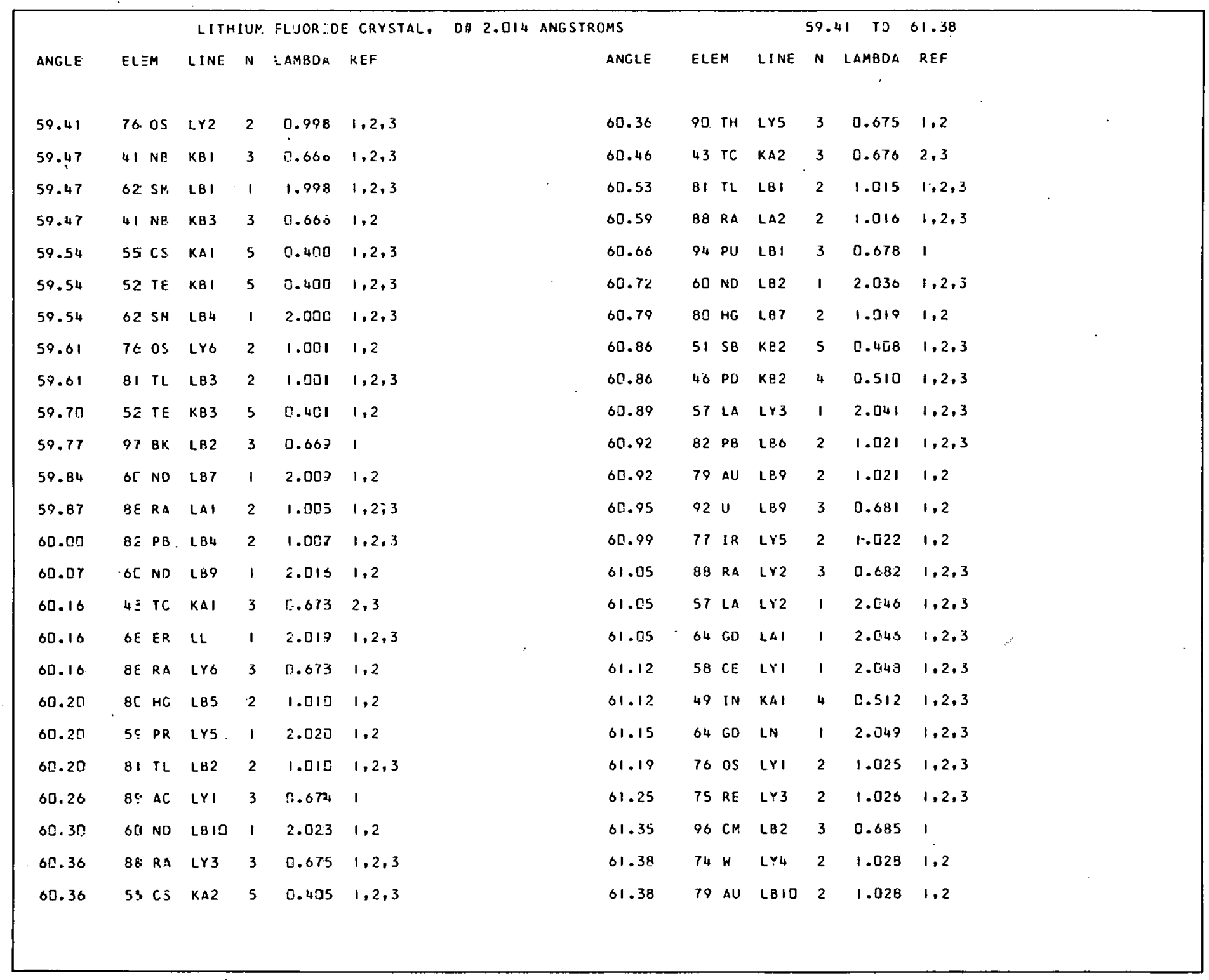




\begin{tabular}{|c|c|c|c|c|c|c|c|c|c|c|c|}
\hline \multirow[b]{2}{*}{ ANGLE } & \multirow[b]{2}{*}{ ELEM } & \multicolumn{2}{|c|}{ LITHIUM } & \multicolumn{2}{|c|}{ FLUORIOE CRYSTAL, } & D\# 2.014 ANGSTROMS & \multicolumn{5}{|c|}{01.42 T0 63.31} \\
\hline & & LINE & N & LAMBDA & REF & ANGLE: & ELEM & LINE & $\mathrm{N}$ & LAMBDA & REF \\
\hline 61.42 & 6460 & LA2 & 1 & 2.057 & $1,2,3$ & 62.35 & $24 \mathrm{CR}$ & KB3 & 1 & 2.085 & 1,2 \\
\hline 6.1 .52 & $98 \mathrm{CF}$ & LYI & 4 & 0.515 & 1 & 62.38 & $67 \mathrm{HO}$ & LL & 1 & 2.086 & $1,2,3$ \\
\hline 61.52 & $87 \mathrm{FR}$ & LAI & 2 & 1.030 & $1,2,3$ & 02.45 & $40 \mathrm{ZR}$ & KBS & 3 & 0.096 & 1 \\
\hline 61.55 & $92 \mathrm{U}$ & LBIO & 3 & 0.687 & 1,2 & 62.45 & $35 \mathrm{BR}$ & KA2 & 2 & 1.044 & $1,2,3$ \\
\hline 61.65 & $75 \mathrm{RE}$ & LY2 & 2 & 1.032 & $1,2,3$ & 62.51 & 33 AS & KB2 & 2 & 1.045 & 1.2 .3 \\
\hline 61.78 & $80 \mathrm{HG}$ & L83 & 2 & 1.034 & $1,2,3$ & 62.51 & 5158 & KB3 & 5 & 0.418 & 1,2 \\
\hline 01.78 & $46 \mathrm{PD}$ & KBS & 4 & 0.517 & 1 & 62.55 & $59 \mathrm{PR}$ & LB7 & 1 & 2.091 & 1.2 \\
\hline 01.78 & $49 \mathrm{IN}$ & KA2 & 4 & 0.517 & $1,2,3$ & 02.65 & $93 \mathrm{NP}$ & LBI & 3 & 0.698 & $1,2,3$ \\
\hline 61.85 & $402 R$ & KB2 & 3 & $\Gamma .690$ & $1,2,3$ & 62.78 & 33 AS & KB5 & 2 & 1.049 & 1,2 \\
\hline 61.85 & $51 \mathrm{SB}$ & KBS & 5 & 0.414 & 1 & 62.78 & $80 \mathrm{HG}$ & L8I & 2 & 1.049 & $1,2,3$ \\
\hline 61.88 & $24 \mathrm{CR}$ & KB5 & 1 & 2.071 & 1,2 & 62.85 & $79 \mathrm{AU}$ & LB7 & 2 & 1.050 & 1,2 \\
\hline 61.98 & $75 \mathrm{RE}$ & LYO & 2 & 1.037 & 1.2 & 62.85 & $59 \mathrm{PR}$ & $\angle B Q$ & 1 & 2.100 & 1.2 \\
\hline 62.01 & $56 \mathrm{BA}$ & LY4 & 1 & 2.075 & 1.2 & 62.85 & $81 \mathrm{TL}$ & LB6 & 2 & 1.050 & $1,2,3$ \\
\hline 62.11 & $81 \mathrm{TL}$ & LB4 & 2 & 1.039 & $1,2,3$ & 62.85 & $54 \times E$ & KA2 & 5 & 0.420 & $1,2,3$ \\
\hline 62.18 & $79 \mathrm{AU}$ & LBS & 2 & 1.040 & 1,2 & 62.91 & $25 \mathrm{MN}$ & KAI & 1 & 2.102 & $1,2,3$ \\
\hline 62.18 & $80 \mathrm{HG}$ & LB2 & 2 & 1.040 & $1,2,3$ & 62.95 & $91 \mathrm{PA}$ & L49 & 3 & 0.701 & 1.2 \\
\hline 62.18 & $54 \times E$ & KAI & 5 & 0.416 & $1,2.3$ & 62.95 & $95 \mathrm{AM}$ & LB2 & 3 & 0.701 & 1,3 \\
\hline 02.18 & $35 \mathrm{BR}$ & KAI & 2 & 1.040 & $1,2,3$ & 62.98 & $60 \mathrm{ND}$ & LB6 & 1 & 2.104 & $1,2.3$ \\
\hline 02.21 & $61 \mathrm{PM}$ & LBI & 1 & 2.081 & $1,2,3$ & 63.05 & $25 \mathrm{MN}$ & KA2 & 1 & 2.106 & $1,2,3$ \\
\hline 62.31 & $46 \mathrm{PD}$ & KB3 & 4 & 0.521 & 1,2 & 63.05 & $40 \mathrm{ZR}$ & KB3 & 3 & 0.702 & 1,2 \\
\hline 62.31 & $46 P D$ & KBI & 4 & 0.521 & $1,2,3$ & 63.25 & $402 R$ & KBI & 3 & 0.702 & $1,2,3$ \\
\hline 62.31 & $87 \mathrm{FR}$ & LAZ & 2 & 1.042 & 1 & 03.08 & $59 \mathrm{PR}$ & L810 & 1 & 2.107 & 1,2 \\
\hline 62.35 & $24 \mathrm{CR}$ & KBI & 1 & 2.085 & $1,2,3$ & 03.11 & $78 \mathrm{PT}$ & L89 & 2 & 1.054 & 1,2 \\
\hline 62.35 & $88 \mathrm{RA}$ & LYI & 3 & 0.695 & $1,2,3$ & 63.18 & $58 \mathrm{CE}$ & LYS & 1 & 2.110 & 1,2 \\
\hline 62.35 & 5158 & KBI & 5 & 0.417 & $1,2,3$ & 63.31 & $33 \mathrm{AS}$ & KBI & 2 & 1.057 & $1,2,3$ \\
\hline
\end{tabular}




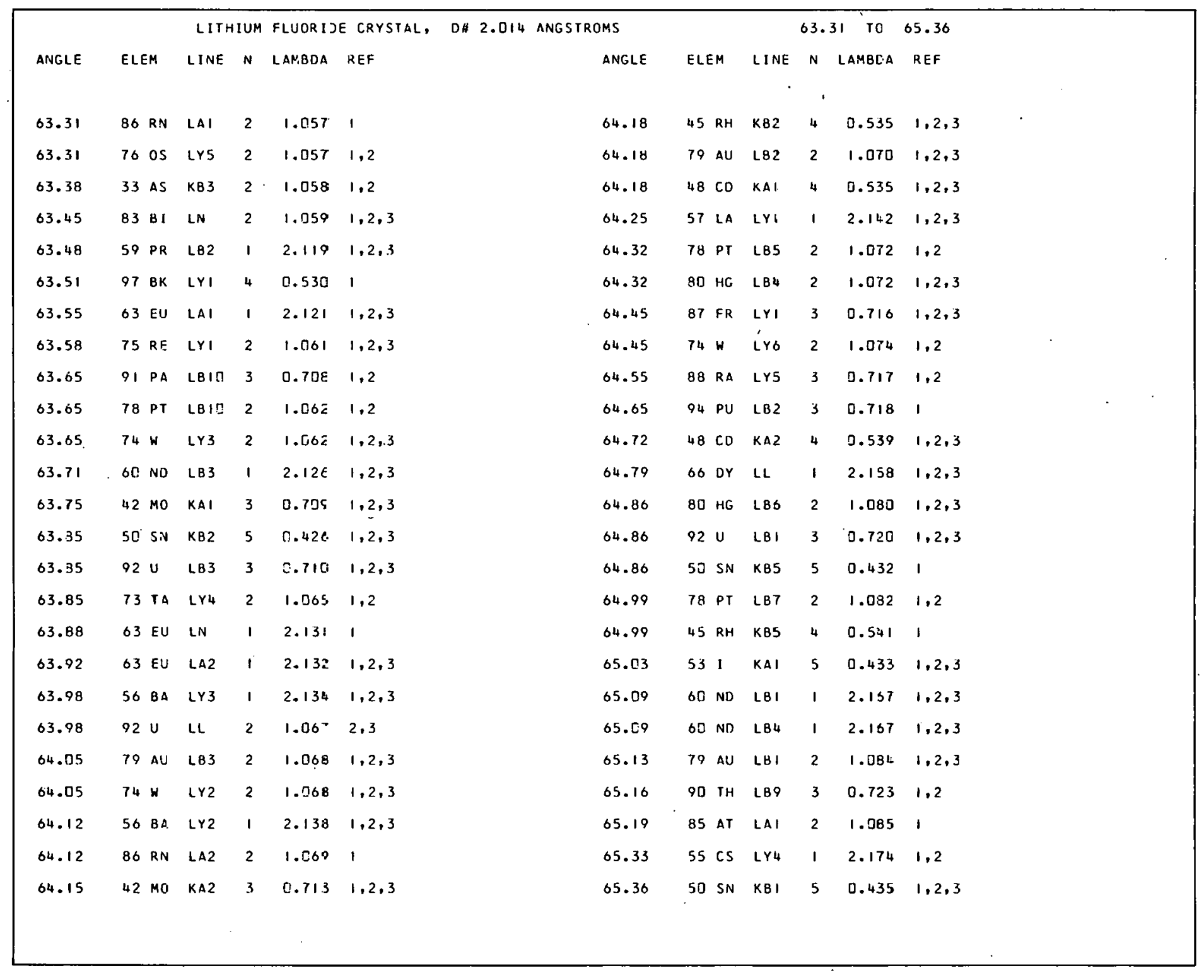




\begin{tabular}{|c|c|c|c|c|c|c|c|c|c|c|c|c|c|c|}
\hline \multirow[b]{2}{*}{ ANGLE } & \multirow{2}{*}{\multicolumn{2}{|c|}{ ELEM. }} & \multicolumn{2}{|c|}{ LITHIUM } & \multicolumn{2}{|c|}{ FLUORIDE CRYSTAL, } & \multirow{2}{*}{$\begin{array}{l}\text { ANGSTROMS } \\
\text { ANGLE }\end{array}$} & \multicolumn{6}{|c|}{65.46 TO 67.33} & \\
\hline & & & LINE & $\mathbf{N}$ & LAMBDA & $0 * 2.014$ & & ELEI & & LINE & $N$ & LAMBDA & REF & \\
\hline 65.46 & 92 & $u$ & L85 & 3 & .0 .726 & 1,2 & 66.38 & 571 & LA & LY5 & 1 & 2.205 & 1,2 & \\
\hline 65.53 & 50 & SN & KB3 & 5 & 0.436 & 1,2 & 66.41 & 72 & $\mathrm{HF}$ & LY4 & 2 & 1.103 & 1,2 & \\
\hline 65.53 & 58 & $C \varepsilon$ & 287 & 1 & 2.180 & 1,2 & 66.43 & 921 & & LB7 & 3 & 0.736 & 1.2 & \\
\hline 65.53 & 77 & IR & LB9 & 2 & 1.090 & 1,2 & 66.48 & 931 & NP & LB2 & 3 & 0.736 & $1,2,3$ & $\cdot$ \\
\hline 65.57 & 39 & Y & KB4 & 3 & 0.727 & 2 & 66.48 & 78 & PT & $L 83$ & 2 & 1.104 & $1,2,3$ & \\
\hline 65.60 & 91 & PA & LL & 2 & 1.091 & $1,2,3$ & 66.52 & 58 & CE & LB2 & 1 & 2.209 & $1,2,3$ & \\
\hline 65.67 & 82 & PB & $L N$ & 2 & 1.092 & $1,2,3$ & 66.55 & 73 & TA & LY2 & 2 & 1.105 & $1,2,3$ & \\
\hline 65.67 & 45 & RH & KBI & 4 & 0.546 & $1,2,3$ & 66.55 & 34 & SE & KAI & 2 & 1.105 & $1,2,3$ & \\
\hline 65.67 & 45 & $\mathrm{RH}$ & KB3 & 4 & 0.546 & 1,2 & 66.55 & 62 & SM & LA2 & 1 & 2.210 & $1,2,3$ & \\
\hline 65.67 & 96 & $C M$ & LYI & 4 & 0.546 & 1 & 66.62 & 77 & IR & LBS & 2 & 1.106 & 1,2 & . \\
\hline 65.77 & 39 & $Y$ & KB2 & 3 & 0.729 & $1,2,3$ & 66.69 & 79 & $A U$ & $\angle 84$ & 2 & 1.107 & $1,2,3$ & \\
\hline 65.80 & 75 & $R E$ & LYS & 2 & 1.094 & 1,2 & 66.79 & 86 & RN & $|y|$ & 3 & 0.739 & 1 & \\
\hline 65.80 & 58 & $\mathrm{CE}$ & 189 & 1 & 2.188 & 1,2 & 66.79 & 59 & $P R$ & LB3 & 1 & 2.217 & $1,2,3$ & \\
\hline 65.87 & 59 & $P R$ & LB6 & 1 & 2.190 & $1,2,3$ & 66.82 & 34 & SE & KA2 & 2 & 1.109 & $1,2,3$ & \\
\hline 65.87 & 53 & I & KA2 & 5 & 0.438 & $1,2,3$ & 66.82 & 62 & 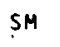 & LN & 1 & 2.218 & $1,2,3$ & \\
\hline 65.87 & 90 & $\mathrm{TH}$ & LBIO & 3 & 0.730 & 1,2 & 66.96 & 79 & $A U$ & $\angle B 6$ & 2 & 1.111 & $1,2,3$ & \\
\hline 66.01 & 85 & $A T$ & LA2 & 2 & 1.097 & 1 & 66.99 & 39 & $y$ & KB 3 & 3 & 0.741 & 1,2 & \\
\hline 66.01 & 77 & $I R$ & LBIO & 2 & 1.097 & 1,2 & 66.99 & 39 & $r$ & KB I & 3 & 0.741 & $1,2,3$ & \\
\hline 66.04 & 58 & $C E$ & LBIO & 1 & 2.195 & 1,2 & 67.06 & 49 & IN & $\mathrm{K} B 2$ & 5 & 0.445 & $1,2,3$ & \\
\hline 66.07 & 91 & PA & L83 & 3 & 0.732 & $1,2,3$ & 67.09 & 91 & $P A$ & LBI & 3 & 0.742 & $1,2,3$ & \\
\hline 66.14 & 73 & TA & LY3 & 2 & 1.099 & $1,2,3$ & 67.16 & 73 & IA & LYo & 2 & 1.114 & 1,2 & \\
\hline 66.14 & 74 & $H$ & LYI & 2 & 1.099 & $1,2,3$ & 67.16 & 84 & Po & $|A|$ & 2 & 1.114 & $1,2,3$ & \\
\hline 66.18 & 62 & $S M$ & LAI & 1 & 2.199 & $1,2,3$ & 67.23 & $9 D$ & $\mathrm{TH}$ & LL & 2 & 1.115 & $1,2,3$ & \\
\hline 66.28 & 39 & $Y$ & $K B 5$ & 3 & 0.734 & 1 & 67.23 & 77 & IR & $\lfloor B 7$ & 2 & 1.115 & 1.2 & \\
\hline 66.35 & 78 & $P T$ & LB2 & 2 & 1.102 & $1,2,3$ & 67.33 & 55 & CS & LY3 & 1 & 2.233 & $1,2,3$ & \\
\hline
\end{tabular}




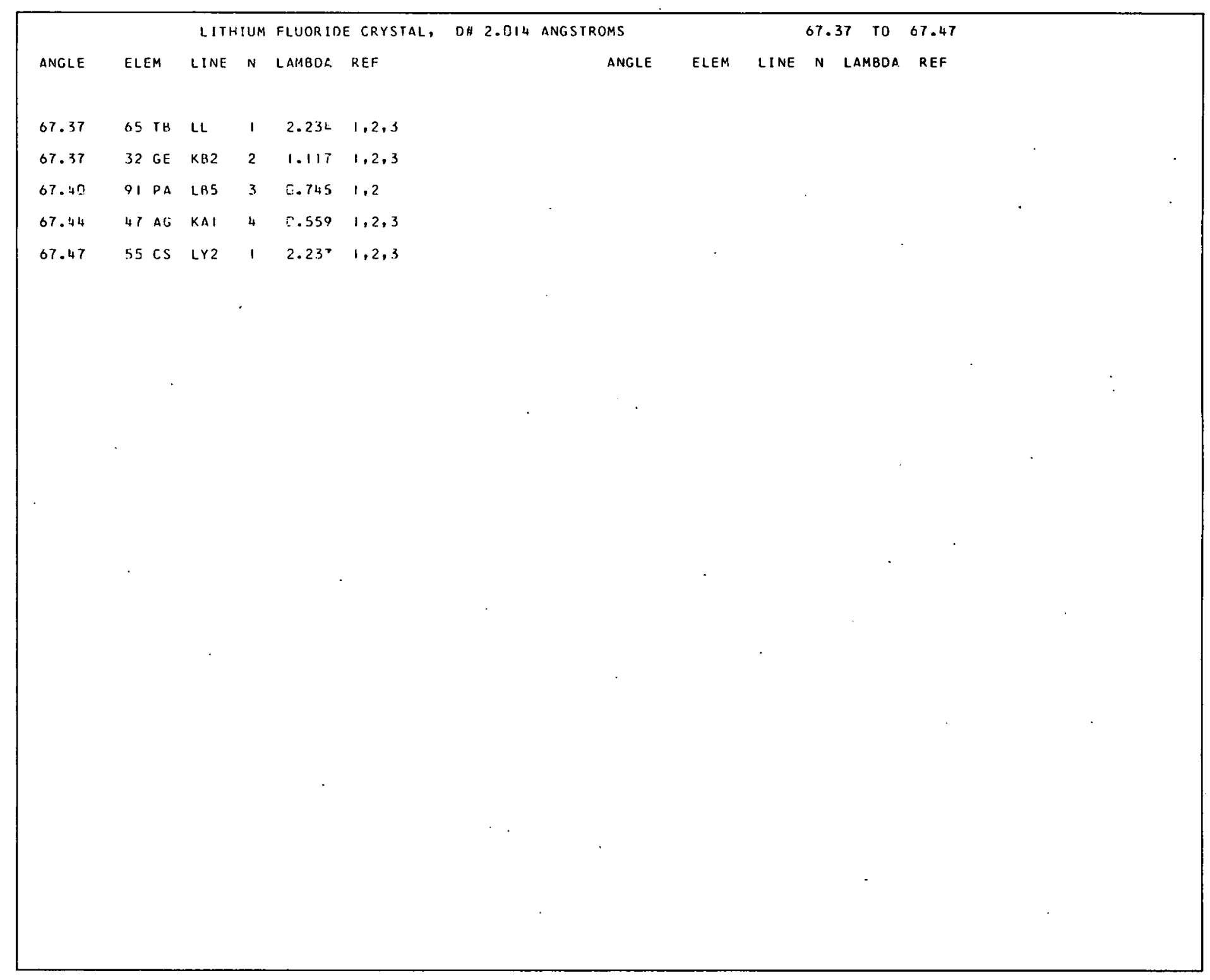




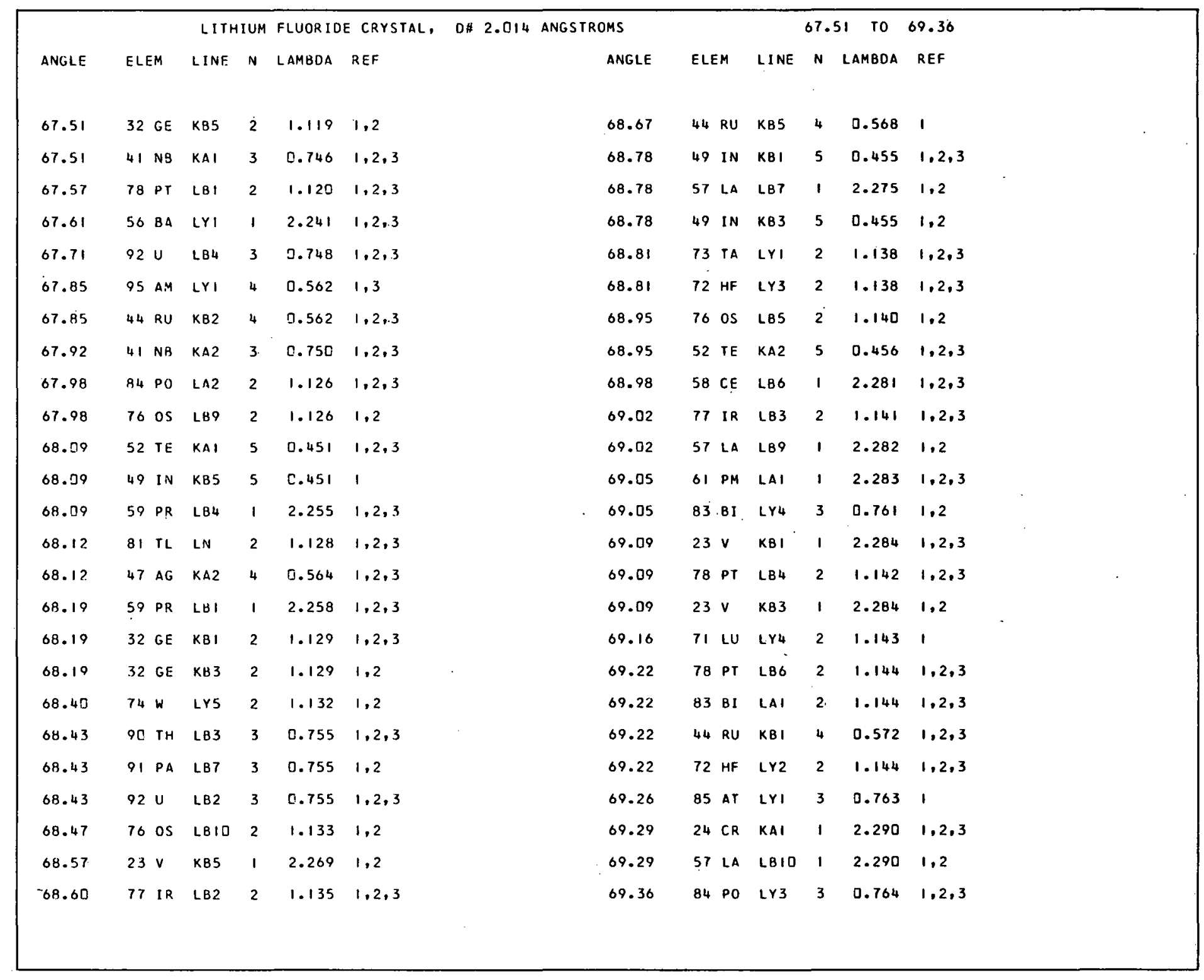




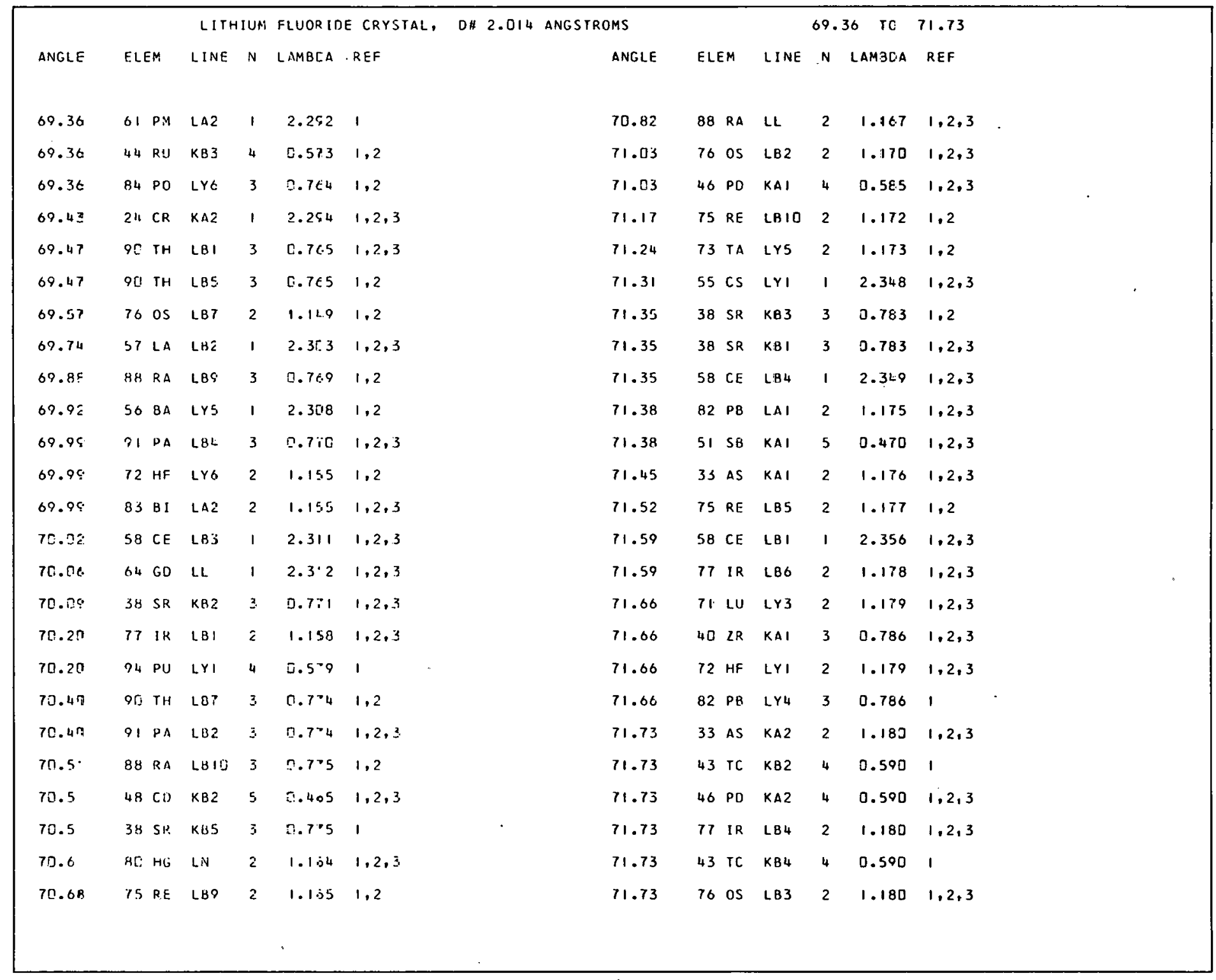




\begin{tabular}{|c|c|c|c|c|c|c|c|c|c|c|c|c|c|c|c|c|}
\hline \multirow[b]{2}{*}{ ANGLE } & \multirow{2}{*}{\multicolumn{2}{|c|}{ ELEM }} & \multicolumn{2}{|c|}{ LITHIUM } & \multirow{2}{*}{$\begin{array}{l}\text { FLUORIOE } \\
\text { LAMBDA }\end{array}$} & \multirow{2}{*}{$\begin{array}{l}\text { E CRYSTAL, } \\
\text { REF }\end{array}$} & \multirow{2}{*}{\multicolumn{2}{|c|}{ D\#2.014 }} & \multirow{2}{*}{$\begin{array}{l}\text { ANGSTROMS } \\
\text { ANGLE }\end{array}$} & \multirow{2}{*}{\multicolumn{2}{|c|}{ ELEM }} & \multicolumn{4}{|c|}{71.77 10 73.85} & \\
\hline & & & LINE & N & & & & & & & & LINE & $\cdot \mathbf{N}$ & LAMBOA & REF & \\
\hline 71.77 & 84 & PO & LYI & 3 & 0.787 & $1,2,3$ & & & 72.82 & 53 & & LY4 & 1 & 2.391 & 1,2 & \\
\hline 71.87 & 92 & & LB6 & 3 & 0.788 & $1,2,3$ & & & 72.86 & 31 & GA & KB2 & 2 & 1.196 & $1,2,3$ & \\
\hline 71.98 & 89 & $A C$ & L甘I & 3 & 0.789 & 1 & & & 72.86 & 92 & $u$ & $\operatorname{LY} 3$ & 4 & 0.598 & 1.2 .3 & \\
\hline 71.98 & 83 & BI & LY3 & 3 & 0.789 & $1,2,3$ & & & 72.93 & 31 & GA & KB5 & 2 & 1.197 & 1,2 & \\
\hline 72.08 & 60 & NO & $\lfloor A \mid$ & 1 & 2.370 & $1,2,3$ & & & 72.93 & 76 & os & LBI & 2 & 1.197 & $1,2,3$ & \\
\hline 72.08 & 71 & LU & LY2 & 2 & 1.185 & $1,2,3$ & & . & 72.97 & 63 & EU & LL & 1 & 2.395 & $1,2,3$ & \\
\hline 72.08 & 70 & $Y_{3}$ & $2 Y_{4}$ & 2 & 1.185 & 1,2 & & & 73.00 & 71 & $\mathrm{LU}$ & LYo & 2 & 1.198 & 1,2 & \\
\hline 72.08 & 83 & BI & LYo & 3 & 0.790 & 1,2 & & & 73.28 & 43 & $T C$ & KB3 & 4 & 0.601 & 1.2 & \\
\hline 72.08 & 40 & $2 R$ & KA2 & 3 & 0.790 & $1,2,3$ & & & 73.28 & 56 & $B A$ & LB2 & 1 & 2.404 & $1,2,3$ & \\
\hline 72.15 & 82 & $P_{B}$ & LA2 & 2 & 1.186 & $1,2,3$ & & & 73.36 & 79 & $A U$ & $L N$ & 2 & 1.203 & $1,2,3$ & \\
\hline 72.15 & 75 & $R E$ & LB7 & 2 & $1.186^{5}$ & 1,2 & & & 73.43 & 74 & $H$ & LB9 & 2 & 1.204 & 1,2 & \\
\hline 72.26 & 48 & CD & KBI & 5 & 0.475 & $1,2,3$ & & & 73.43 & 43 & TC & KBI & 4 & 0.602 & $1,2,3$ & \\
\hline 72.26 & 51 & $S H$ & KA2 & 5 & 0.475 & $1,2.3$ & & & 73.46 & 60 & ND & LN & 1 & 2.409 & $1,2,3$ & \\
\hline 72.30 & 91 & PA & $2 Y_{4}$ & 4 & 0.594 & 1,2 & & & 73.46 & 88 & $R A$ & L.B3 & 3 & 0.803 & $1,2,3$ & \\
\hline 72.30 & 56 & $B A$ & LB9 & 1 & 2.376 & 1,2 & & & 73.50 & 57 & LA & 283 & 1 & 2.410 & $1,2,3$ & \\
\hline 72.30 & 98 & $\mathrm{CF}$ & $\lfloor A \mid$ & 3 & 0.792 & 1 & & & 73.57 & 98 & $C F$ & LBI & 4 & 0.603 & 1 & \\
\hline 72.40 & 90 & $T_{H}$ & LB4 & 3 & 0.793 & $1,2,3$ & & & 73.57 & 98 & $C F$ & LA2 & 3 & 0.804 & 1 & \\
\hline 72.40 & 57 & LA & LHO & 1 & 2.379 & $1,2,3$ & & & 73.64 & 75 & RE & LB2 & 2 & 1.207 & $1,2,3$ & \\
\hline 72.44 & 60 & ND & LA2 & 1 & 2.380 & 1.3 & & & 73.64 & 81 & $\mathrm{TL}$ & LAI & 2 & 1.207 & $1,2,3$ & \\
\hline 72.44 & 48 & $C D$ & KB3 & 5 & 0.476 & 1,2 & & & 73.68 & 92 & $u$ & $\ln$ & 3 & 0.805 & $1,2,3$ & \\
\hline 72.44 & 92 & $u$ & LYo & 4 & 0.595 & 1,2 & & & 73.71 & 31 & GA & KBI & 2 & 1.208 & $1,2,3$ & \\
\hline 72.51 & 90 & $T H$ & LB2 & 3 & 0.794 & $1,2,3$ & & & 73.71 & 31 & $G A$ & $K B 3$ & 2 & 1.208 & 1,2 & \\
\hline 72.68 & 56 & $B A$ & LBIT & 1 & 2.387 & 1,2 & & & 73.75 & 55 & $c s$ & LYS & 1 & 2.417 & 1,2 & \\
\hline 72.72 & 83 & 81 & $\operatorname{LY} 2$ & 3 & 0.796 & $1,2,3$ & & & 73.78 & 88 & $R \dot{A}$ & L.B5 & 3 & 0.806 & 1.2 & \\
\hline 72.72 & 93 & $N P$ & LY & 4 & 0.597 & $1,2,3$ & & & 73.85 & 92 & $u$ & LY2 & 4 & 0.605 & $1,2,3$ & - \\
\hline
\end{tabular}




\begin{tabular}{|c|c|c|c|c|c|c|c|c|c|c|c|c|c|c|c|}
\hline & & & LITH & IUM & FLUORIDE & E CRYSTAL, & DA 2.014 & ANGSTROMS & & & & 74.0 & TO & 76.15 & \\
\hline ANGLE & ELEM & EM & LINE & $\mathbf{N}$ & $\angle A .4 B D A$ & REF & & ANGLE & ELEF & $E M$ & LINE & $N$ & LAMBDA & REF & \\
\hline 74.00 & $74 k$ & $W$ & LB 10 & 2 & 1.212 & 1.2 & & 75.00 & 918 & PA & LYo & 4 & 0.613 & 1,2 & \\
\hline 74.00 & $91 P$ & $P A$ & LB6 & 3 & 0.808 & $1,2,3$ & & 75.00 & $45 R$ & RH & KAI & 4 & 0.613 & $1,2,3$ & \\
\hline 74.07 & 760 & OS & LBG & 2 & 1.213 & $1,2,3$ & & 75.10 & $50 \mathrm{~s}$ & SN & KAI & 5 & 0.491 & $1,2.3$ & \\
\hline 74.21 & 74 & $w$ & L85 & 2 & 1.215 & 1,2 & & 75.21 & $70 \mathrm{r}$ & Y8 & $\operatorname{LY} 2$ & 2. & 1.229 & $1,2,3$ & \\
\hline 74.21 & 72 & HF & LY5 & 2 & 1.215 & 1.2 & & 75.21 & 57 & LA & LEI & 1 & 2.458 & $1,2,3$ & \\
\hline 74.21 & 978 & BK. & LA & 3 & 0.910 & 1 & & 75.21 & 691 & $T M$ & $\operatorname{Lr} 4$ & 2 & 1.220 & 1 & \\
\hline 74.39 & $47 \mathrm{~A}$ & $A G$ & KB2 & 5 & 0.487 & $1,2,3$ & & 75.28 & 921 & $u$ & $1 . Y 1$ & 4 & 0.615 & $1,2,3$ & \\
\hline 74.42 & 811 & TL & LY4 & 3 & 0.812 & 1,2 & & 75.36 & $54 x$ & $X E$ & LYI & 1 & 2.462 & 1 & \\
\hline 74.42 & 760 & os & LB4 & 2 & 1.213 & $1,2,3$ & & 75.39 & 82. & PB & LY2 & 3 & 0.821 & $1,2,3$ & \\
\hline 74.50 & 817 & TL & LA2 & 2 & 1.217 & $1,2,3$ & & 75.39 & $59 \mathrm{~F}$ & $P R$ & LAI & 1 & 2.463 & $1,2,3$ & \\
\hline 74.53 & 831 & B $:$ & $|Y|$. & 3 & 0.813 & $1,2,3$ & & 75.46 & 47 & $A G$ & K65 & 5 & 0.493 & 1 & \\
\hline 74.57 & $75 F$ & $R E$ & LB3 & 2 & $1.22 \mathrm{E}$ & $1,2,3$ & & 75.50 & $37 \mathrm{~F}$ & RB & KBS & 3 & 0.822 & 1 & \\
\hline 74.64 & $88 \mathrm{~F}$ & RA & LB I & 3 & 0.814 & $1,2,3$ & & 75.50 & 97 & $B K$ & LA2 & 3 & 0.822 & 1 & \\
\hline 74.64 & 89 & $A C$ & LB2 & 3 & 0.814 & 1 & & 75.57 & 915 & $P A$ & LY3 & 4 & 0.617 & $1,2,3$ & \\
\hline 74.71 & 712 & LU & $\mid Y Y$ & 2 & 1.222 & $1,2,3$ & & 75.72 & $45 F$ & $\mathrm{RH}$ & KA2 & 4 & 0.618 & $1,2,3$ & \\
\hline 74.71 & $70 r$ & $Y B$ & LY3 & 2 & 1.222 & $1,2,3$ & & 75.75 & 59 & $P R$ & LA2 & 1 & 2.473 & $1,2,3$ & \\
\hline 74.71 & 901 & $\mathrm{TH}$ & LY4 & 4 & 0.611 & 1,2 & & 75.82 & 50 & $S N$ & KA2 & 5 & 0.495 & $1,2,3$ & 1 \\
\hline 74.75 & $82 F$ & $P 3$ & LY 3 & 3 & 0.815 & $1,2,3$ & & 75.93 & 55 & CS & L89 & 1 & 2.478 & 1,2 & \\
\hline 74.82 & 53 & 1 & LY3 & 1 & 2.447 & 1,2 & & 75.93 & 75 & RE & LBI & 2 & 1.239 & $1,2,3$ & \\
\hline 74.82 & 53 & I & LY2 & 1 & 2.447 & $1,2,3$ & & 76.00 & 421 & мо & KB 4 & 4 & 0.020 & 2 & $\cdot$ \\
\hline 74.85 & $88 \mathrm{~F}$ & RA & LB7 & 3 & 0.816 & 1,2 & & 76.08 & 62 & SM & LL & 1 & 2.482 & $1,2,3$ & \\
\hline 74.85 & 37 & $\mathrm{RA}$ & KB2 & 3 & 0.816 & $1,2,3$ & & 76.08 & 56 & $B A$ & L86 & 1 & 2.482 & $1,2,3$ & \\
\hline 74.85 & 74 & $W$ & $\angle B\}$ & 2 & 1.224 & 1,2 & & 76.08 & 80 & $H G$ & LAI & 2 & 1.241 & $1,2,3$ & \\
\hline 74.85 & 57 & $L A$ & $\mathrm{LB}_{4}$ & 1 & 2.449 & $1,2,3$ & & 76.15 & 421 & MO & KB2 & 4 & 0.621 & $1,2,3$ & \\
\hline 74.98 & 82 & $P_{8}$ & LYG & 3 & 0.817 & 1,2 & & 76.15 & 97 & BK & LBI & 4 & 0.621 & 1 & \\
\hline
\end{tabular}




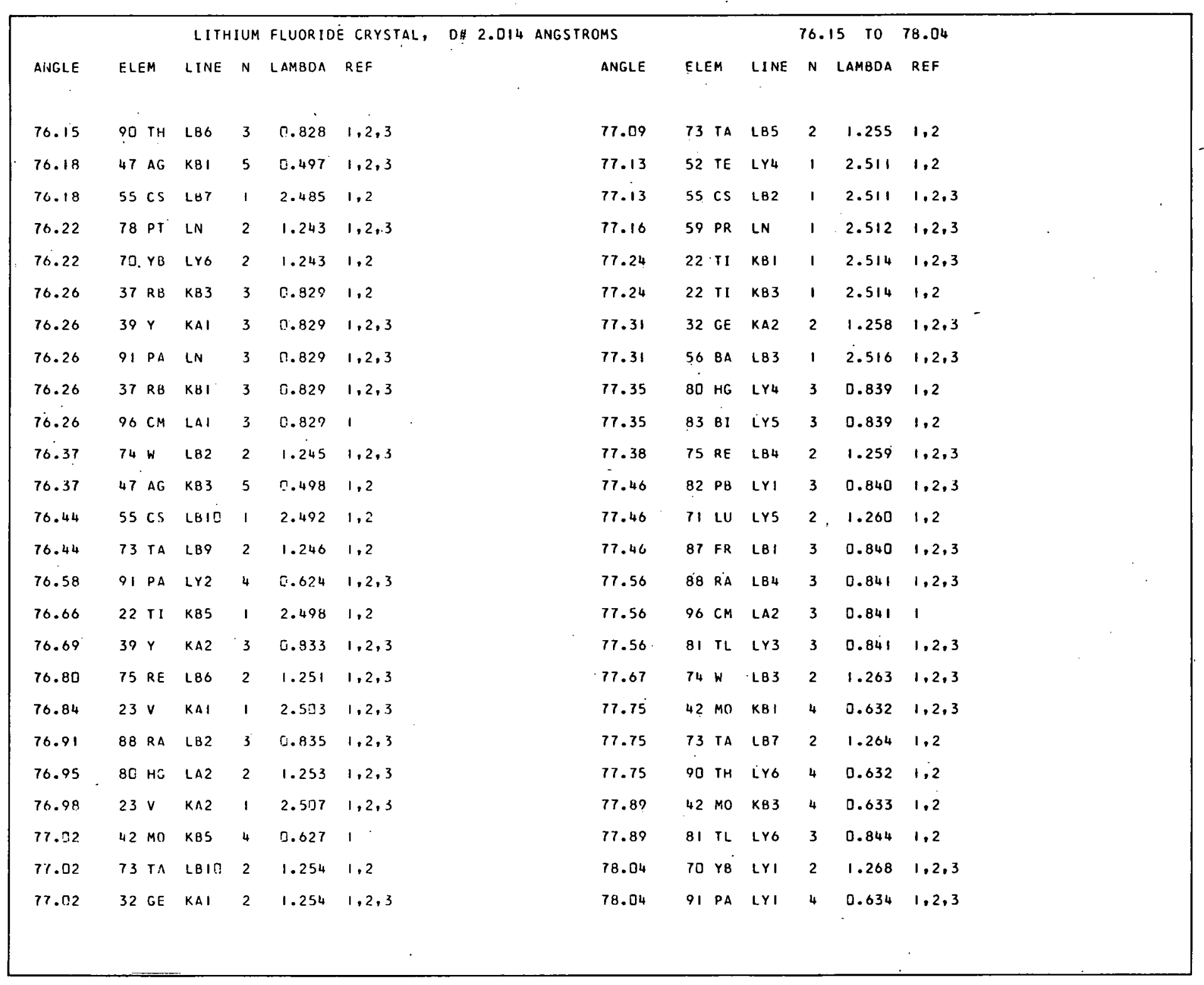




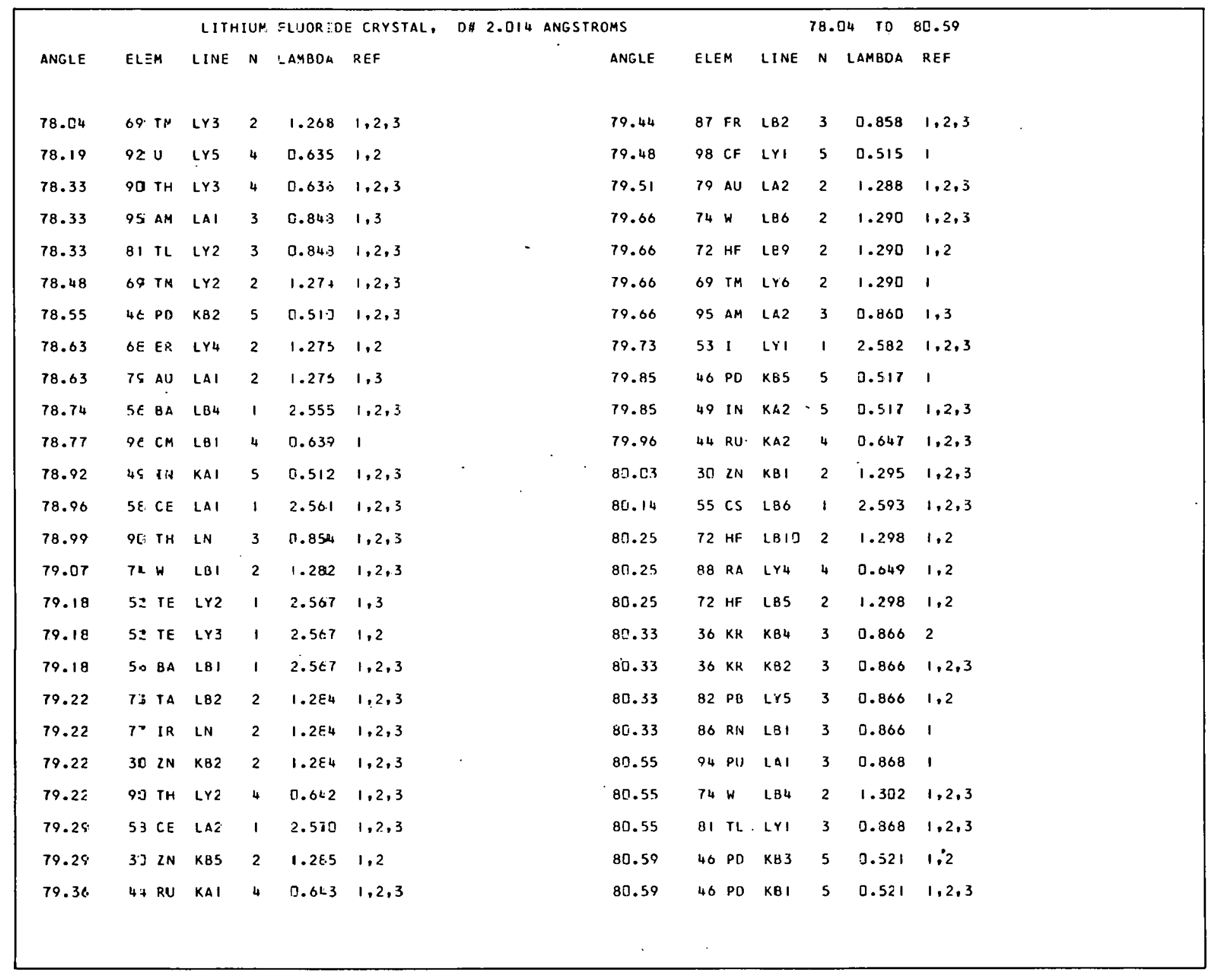




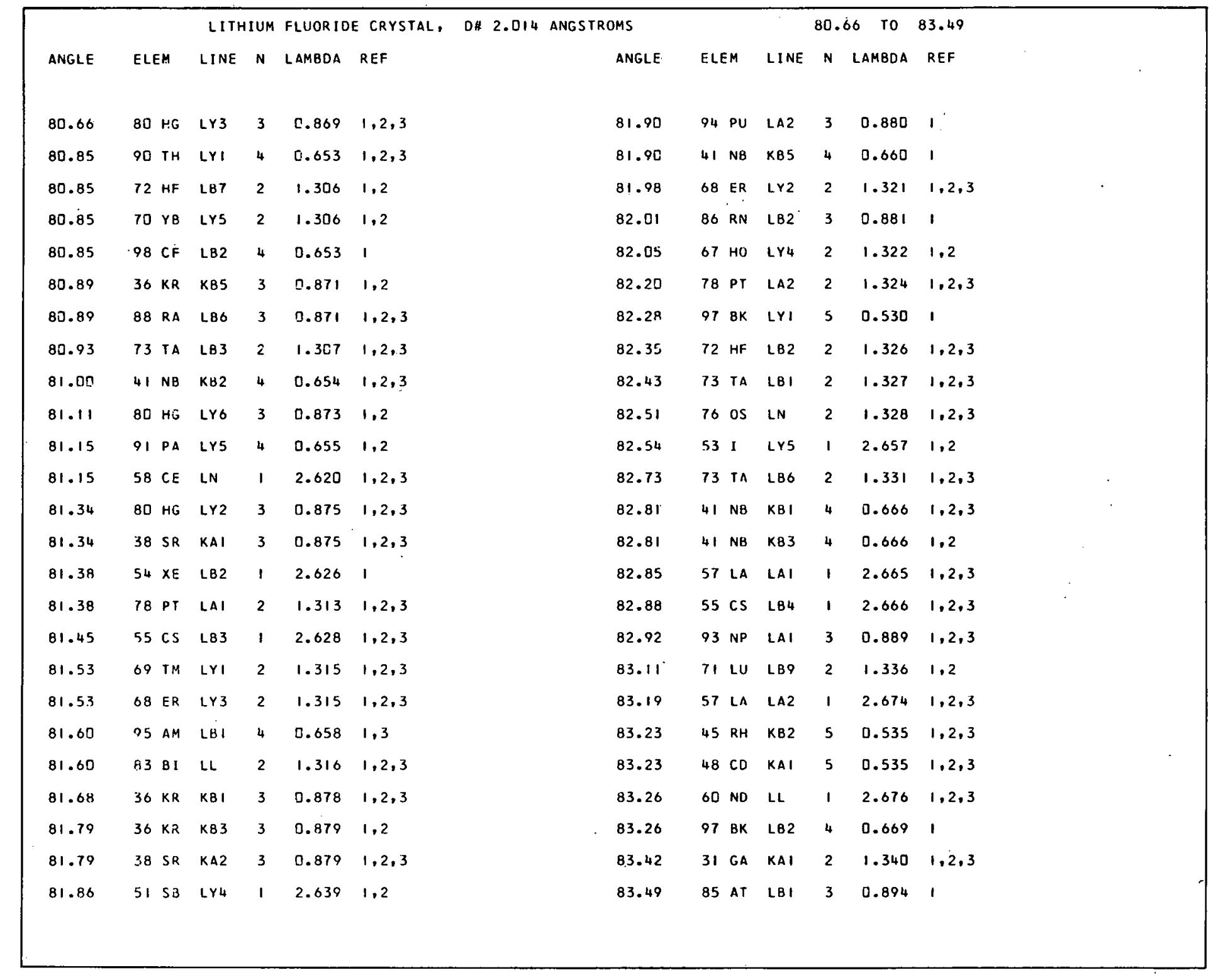




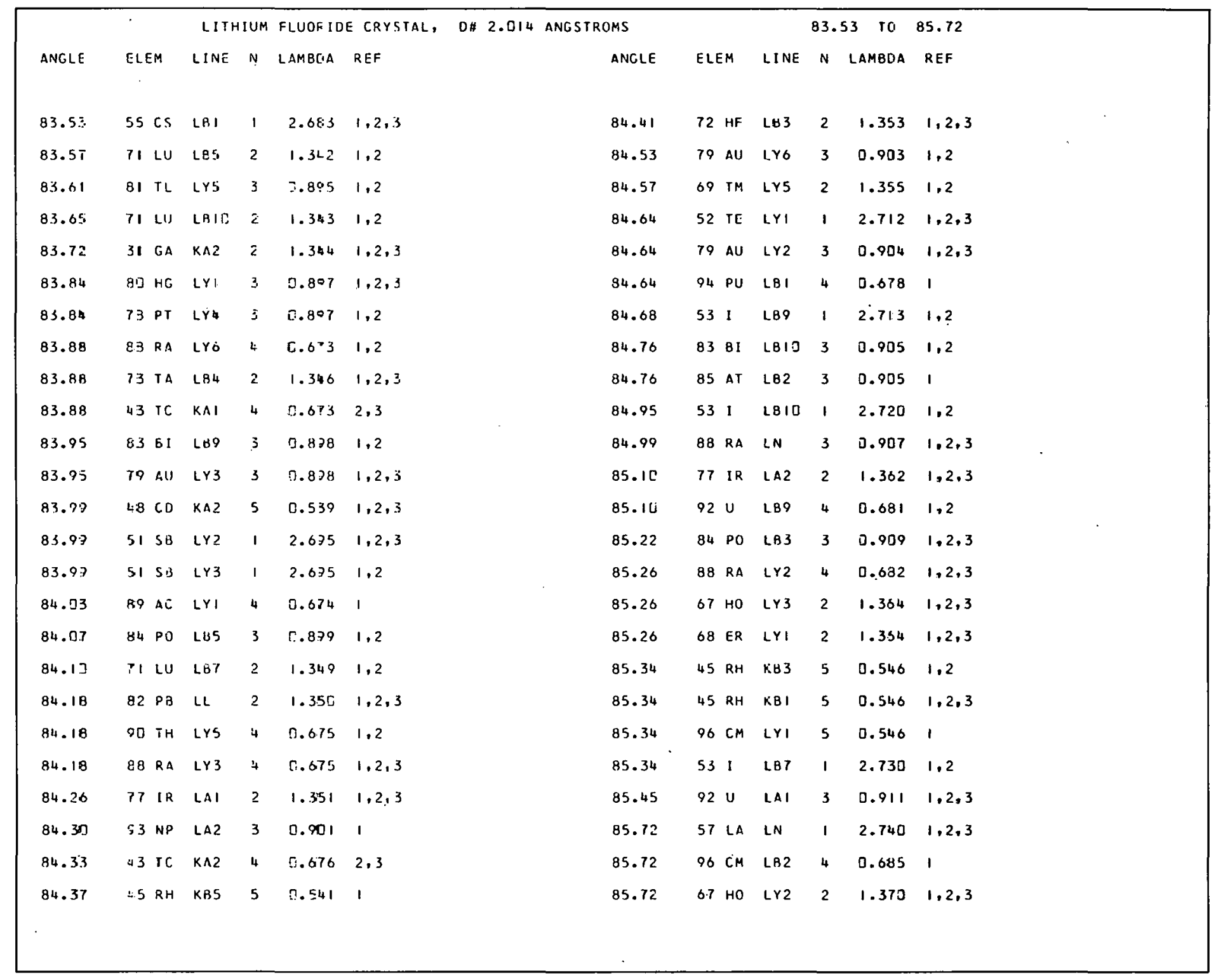




\begin{tabular}{|c|c|c|c|c|c|c|c|c|c|c|c|c|c|c|}
\hline \multirow[b]{2}{*}{ ANGLE } & \multirow[b]{2}{*}{ ELEM } & \multicolumn{2}{|c|}{ LITHIUM. } & \multicolumn{2}{|c|}{ FLUORIDE CRYSTAL, } & \multirow{2}{*}{\multicolumn{2}{|c|}{$\begin{array}{l}\text { DH } 2.014 \text { ANGSTROMS } \\
\text { ANGLE }\end{array}$}} & \multirow{2}{*}{\multicolumn{2}{|c|}{ ELEM }} & \multicolumn{3}{|c|}{85.72 TO } & \multirow{2}{*}{$\begin{array}{l}88.15 \\
\text { REF }\end{array}$} & \\
\hline & & LINE & N & LAMBOA & REF & & & & & LINE & $N$ & LAMBDA & & \\
\hline 85.72 & $71 \mathrm{LU}$ & LB2 & 2 & 1.370 & $1,2,3$ & & 87.29 & 21 & sc & KBI & 1 & 2.780 & $1,2,3$ & \\
\hline 85.96 & $75 \mathrm{RE}$ & LN & 2 & 1.373 & $1,2,3$ & & 87.29 & 88 & RA & $|Y|$ & 4 & 0.695 & $1,2,3$ & \\
\hline 86.03 & 66 DY & $\mathrm{Lr}_{4}$ & 2 & 1.374 & 1,2 & & 87.33 & 77 & I R & LY4 & 3 & 0.927 & 1,2 & \\
\hline 86.03 & 920 & โ8I] & 4 & 0.687 & 1,2 & & 87.33 & 82 & PB & L89 & 3 & 0.927 & 1,2 & \\
\hline 86.03 & $72 \mathrm{HF}$ & LBI & 2 & 1.374 & $1,2,3$ & & 87.33 & 79 & $A U$ & LYI & 3 & 0.927 & $1,2,3$ & \\
\hline 86.03 & $22 \mathrm{TI}$ & KAI & 1 & 2.748 & $1,2,3$ & & 87.37 & 76 & os & LAI & 2 & 1.391 & $1,2,3$ & \\
\hline 86.03 & $72 \mathrm{HF}$ & LB6 & 2 & 1.374 & $1,2.3$ & & 87.37 & 70 & YB & LBIO & 2 & 1.391 & 1,2 & \\
\hline 86.11 & 531 & LB2 & 1 & 2.750 & $1,2,3$ & & 87.44 & 72 & $\mathrm{HF}$ & LB4 & 2 & 1.392 & $1,2,3$ & \\
\hline 86.19 & $22 \mathrm{TI}$ & KA2 & 1 & 2.752 & $1,2,3$ & & -87.44 & 59 & $P R$ & LL & 1 & 2.784 & $1,2,3$ & \\
\hline 86.50 & $402 R$ & Кв 2 & 4 & 0.690 & $1,2,3$ & & 87.44 & 40 & $2 R$ & KB5 & 4 & 0.696 & 1 & \\
\hline 86.58 & $29 \mathrm{CII}$ & КВ2 & 2 & 1.381 & 2,3 & & 87.44 & 78 & PT & $\operatorname{LY} 3$ & 3 & 0.928 & $1,2,3$ & \\
\hline 86.62 & $35 \mathrm{BH}$ & $\mathrm{XB2}$ & 3 & 0.921 & $1,2,3$ & & 87.44 & 29 & $\mathrm{Cu}$ & KBI & 2 & 1.392 & $1,2,3$ & \\
\hline 86.66 & $29 \mathrm{cu}$ & $K \otimes 5$ & 2 & 1.382 & 2 & & 87.48 & 56 & $B A$ & LA? & 1 & 2.785 & $1,2,3$ & \\
\hline 86.66 & $21 \mathrm{SC}$ & KB5 & 1 & 2.764 & 1,2 & & 87.52 & 29 & $\mathrm{cu}$ & KB3 & 2 & 1.393. & 1,2 & \\
\hline 86.74 & $84 \mathrm{PO}$ & LBI & 3 & 0.922 & $1,2,3$ & & 87.56 & 84 & Po & LB 2 & 3 & 0.929 & $1,2,3$ & \\
\hline 86.74 & $92 \mathrm{U}$ & LA2 & 3 & 0.922 & $1,2,3$ & & 87.68 & .52 & TE & LYS & 1 & 2.790 & 1,2 & \\
\hline 86.82 & $70 Y B$ & L89 & 2 & 1.384 & 1,2 & & 87.68 & 37 & $R B$ & KA2 & 3 & 0.930 & $1,2,3$ & \\
\hline 86.89 & $81 \mathrm{TL}$ & LL & 2 & 1.385 & $1,2,3$ & & 87.68 & $70^{\circ}$ & YB & $\lfloor B 7$ & 2 & 1.395 & 1,2 & \\
\hline 86.97 & $80 \mathrm{HG}$ & LYS & 3 & 0.924 & 1,2 & & 87.76 & 93 & NP & LBI & 4 & 0.698 & $1,2,3$ & \\
\hline 87.05 & $70 \mathrm{YB}$ & LB5 & 2 & 1.387 & 1,2 & & 87.88 & 47 & $A G$. & KAI & 5 & 0.559 & $1,2,3$ & \\
\hline 87.09 & $56 \mathrm{BA}$ & $|A|$ & 1 & 2.775 & $1,2,3$ & & 88.04 & 35 & $B R$ & KB I & 3 & 0.933 & $1,2,3$ & \\
\hline 87.09 & $35 \mathrm{BR}$ & KBS & 3 & 0.925 & 1,2 & & 88.04 & 35 & $B R$ & KB3 & 3 & 0.933 & 1,2 & \\
\hline 87.17 & $50 \mathrm{SN}$ & $L_{Y 4}$ & 1 & 2.777 & 1,2 & & 88.04 & 91 & PA & $|A|$ & 3 & 0.933 & $1,2,3$ & \\
\hline 87.21 & 8381 & LOS & 3 & 0.926 & 1,2 & & 88.15 & 71 & LU & LB 3 & 2 & 1.401 & $1,2,3$ & \\
\hline 87.21 & $37 \mathrm{RH}$ & KAI & 3 & 0.926 & $1,2,3$ & & 88.15 & 82 & PB & LBIO & 3 & 0.934 & 1,2 & \\
\hline
\end{tabular}




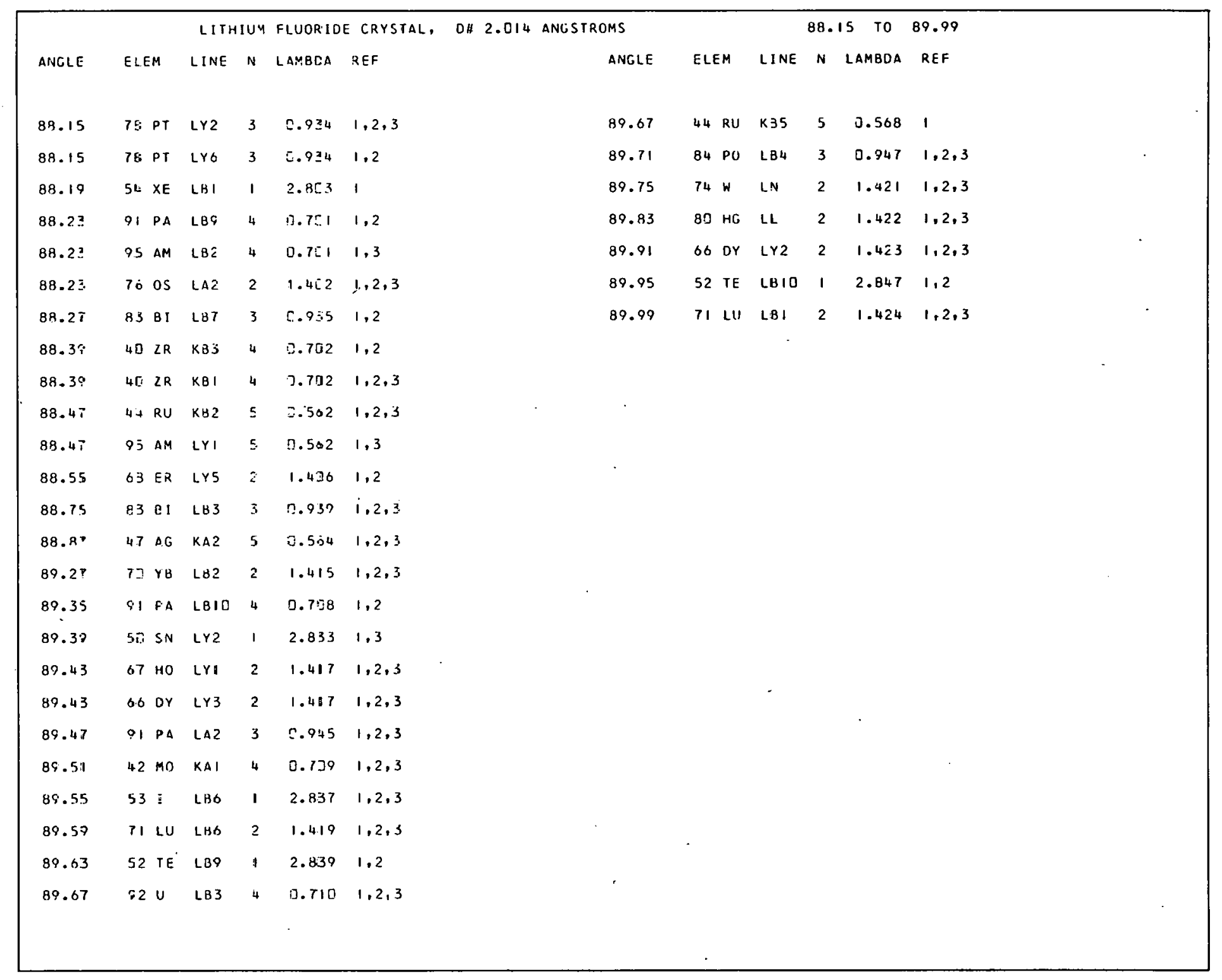




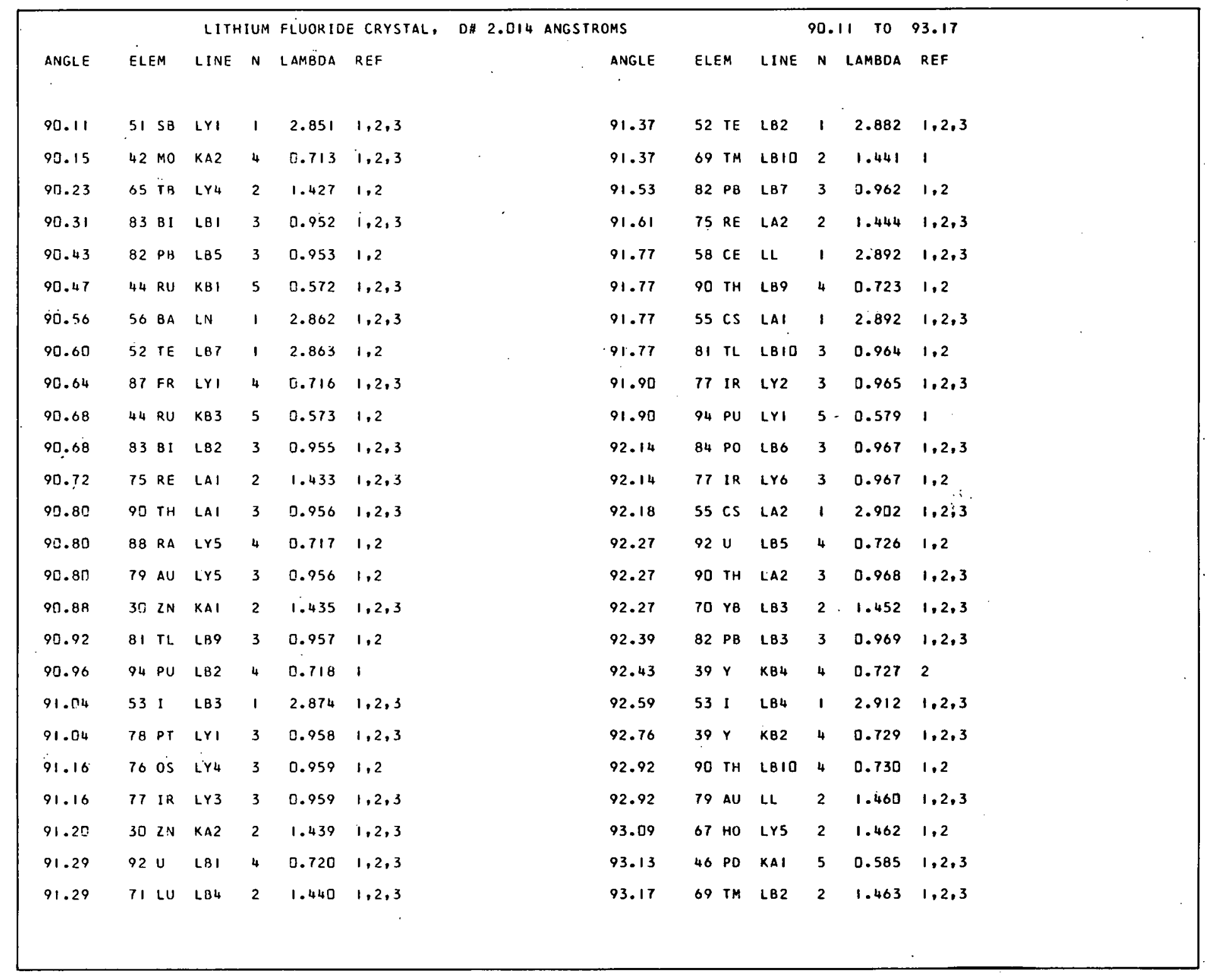




\begin{tabular}{|c|c|c|c|c|c|c|c|c|c|c|c|c|c|c|c|}
\hline & & & LITH & IUM & FLUORIDE & E CRYSTAL, & D\# 2.014 & ANGSTROMS & & & & 93. & $17 \quad 10$ & 95.39 & \\
\hline ANGLE & ELI & & LINE & N & LAMBDA & KEF & & ANGLE & ELE & EM & LINE & N & LAMBDA & REF & \\
\hline 93.17 & 49 & In & $L Y_{4}$ & 1 & 2.925 & 1,2 & & 74.34 & 65 & TB & LY 2 & 2 & 1.477 & $1,2,3$ & \\
\hline 93.26 & 91 & PA & 283 & 4 & 0.732 & $1,2,3$ & & 94.42 & 86 & RN & LYI & 4 & 0.739 & 1 & - \\
\hline 93.38 & 83 & 8[ & LB4 & 3 & $\Upsilon .977$ & $1,2,3$ & & 94.42 & 71 & Lu & LN & 2 & 1.478 & 1,2 & \\
\hline 93.42 & 51 & $S B$ & LYS & 1 & 2.932 & 1,2 & & 94.51 & 80 & $H G$ & LE9 & 3 & 0.986 & 1,2 & \\
\hline 93.42 & 70 & YB & $\angle B 6$ & 2 & 1.463 & $1,2,3$ & & 94.76 & 78 & PT & LY5 & 3 & 0.983 & 1,2 & \\
\hline 93.59 & 30 & Y & KA5 & 4 & 0.734 & 1 & & 94.76 & 39 & $Y$ & $K 63$ & 4 & 0.741 & 1,2 & \\
\hline 93.63 & 53 & I & LBI & 1 & 2.937 & $1,2,3$ & . & 94.76 & 39 & $Y$ & K\$I & 4 & 0.741 & $1,2,3$ & \\
\hline 93.75 & 34 & $S E$ & KB2 & 3 & 0.980 & $1,2,3$ & & 94.93 & 91 & PA & LUI & 4 & 0.742 & $1,2,3$ & \\
\hline 93.75 & 89 & $A C$ & $|A|$ & 3 & {$[.980$} & 1 & & 95.01 & 91 & $P A$ & LY4 & 5 & 0.594 & 1,2 & \\
\hline 93.75 & 36 & Ke & KAI & 3 & 0.980 & $1,2,3$ & & 95.01. & 68 & ER & LB9 & 2 & 1.485 & 1,2 & \\
\hline 93.84 & 65 & $T B$ & $L Y 3$ & 2 & 1.471 & $1,2,3$ & & 95.01 & 81 & $\mathrm{rL}$ & LBT & 3 & 0.990 & 1.2 & \\
\hline 93.84 & 73 & TI & LN & 2 & 1.471 & $1,2,3$ & & 95.01 & 64 & GD & $\mathrm{LY}_{4}$ & 2 & 1.485 & 1,2 & \\
\hline 93.88 & 81 & $\mathrm{TL}$ & L85 & $\dot{3}$ & 5.9881 & 1,2 & & 95.05 & 52 & $\mathrm{TE}$ & LB6 & 1 & 2.971 & $1,2,3$ & \\
\hline 93.92 & 93 & $N ?$ & $\mathrm{LO} 2$ & 4 & 0.736 & $1,2,3$ & & 95.59 & 51 & $S B$ & LB,9 & 1 & 2.972 & 1,2 & \\
\hline 93.92 & 92 & u & $\lfloor\forall 7$ & 4 & R. 736 & 1,2 & & 95.14 & 77 & IR & $\lfloor Y)$ & 3 & 0.991 & $1,2,3$ & \\
\hline 94.00 & 60 & DY & LYI & 2 & 1.473 & $1,2,3$ & & 95.18 & 74 & $w$ & LA2 & 2 & 1.487 & $1,2,3$ & \\
\hline $94.5 \Pi$ & 82 & PB & LB1 & 3 & 0.922 & $1,2,3$ & & 95.22 & 92 & $u$ & LYG & 5 & 0.595 & 1.2 & \\
\hline 94.13 & 82 & PB & LB2 & 3 & 0.983 & $1,2,3$ & & 95.26 & 89 & $A C$ & LA2 & 3 & 0.992 & 1 & \\
\hline 94.17 & 43 & $T C$ & $\mathrm{~KB} 4$ & 5 & ח.550 & 1 & & 95.26 & 76 & os & $L \times 3$ & 3 & 0.092 & $1,2,3$ & \\
\hline 94.17 & 46 & $P D$ & KA2 & 5 & 0.580 & $1,2,3$ & & 95.20 & 34 & SE & $K B I$ & 3 & 0.992 & $1,2.3$ & \\
\hline 94.17 & 43 & TC & $\mathrm{KBz}$ & 5 & 0.590 & 1 & & 95.26 & 75 & RE & $\operatorname{Lr} 4$ & 3 & 0.992 & 1.2 & \\
\hline 94.25 & 75 & YB & LBI & 2 & $1.4 \pi 6$ & $1,2,3$ & & 95.35 & 28 & $\mathrm{NI}$ & K35 & 2 & 1.489 & 1,2 & . \\
\hline 94.25 & 34 & SE & $K B 5$ & 3 & $\because .9 \varepsilon 4$ & 1,2 & & 95.35 & 28 & $\mathrm{NI}$ & $K 32$ & 2 & .1 .489 & $1,2,3$ & \\
\hline 94.25 & 74 & $w$ & LAI & 2 & 1.476 & $1,2,3$ & & 95.39 & 34 & SE & K33 & 3 & 0.093 & 1,2 & \\
\hline 94.25 & 36 & $K R$ & KA2 & 3 & $\Gamma .96 .4$ & $1,2,3$ & & 95.34 & 51 & 58 & LBIE & 1 & 2.979 & 1,2 & \\
\hline
\end{tabular}




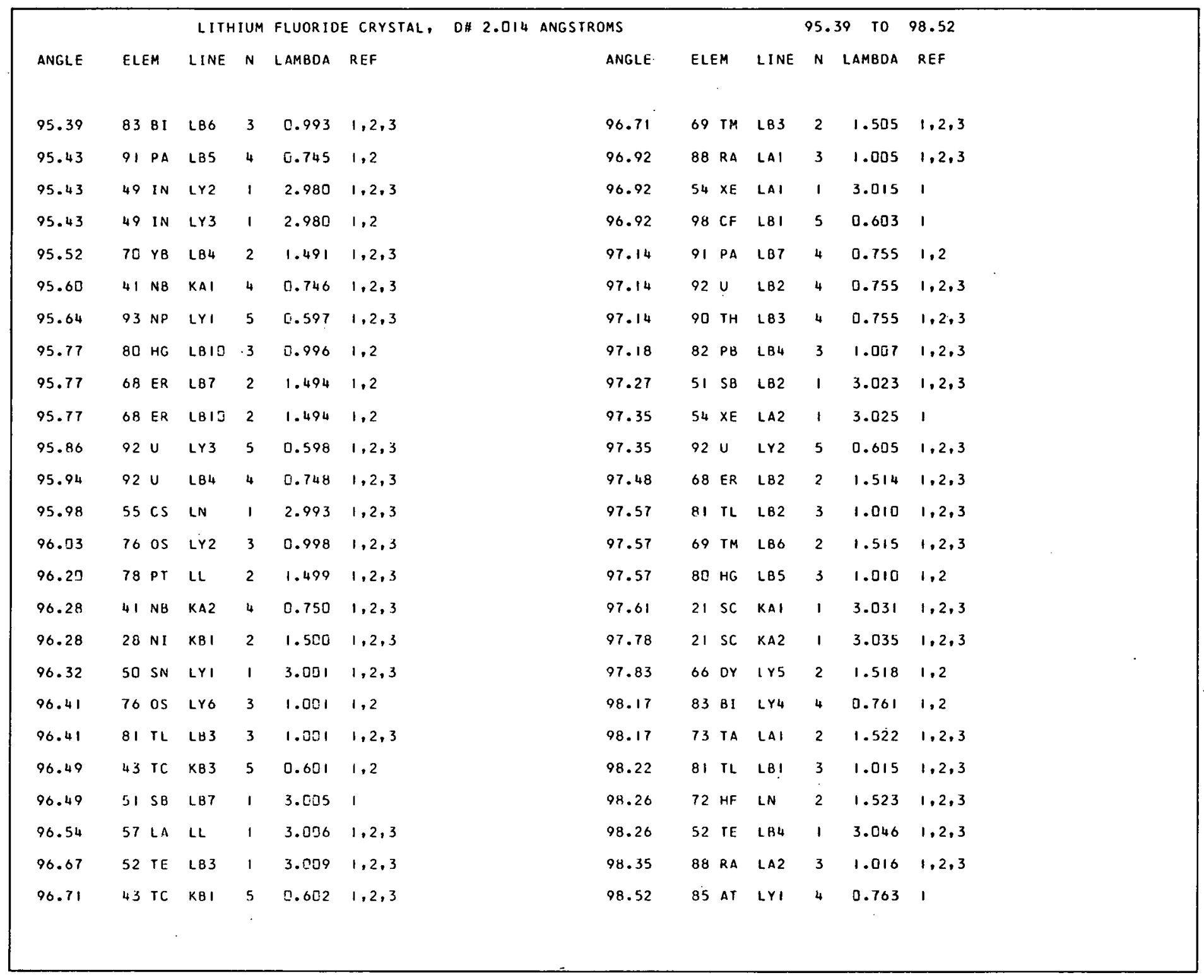




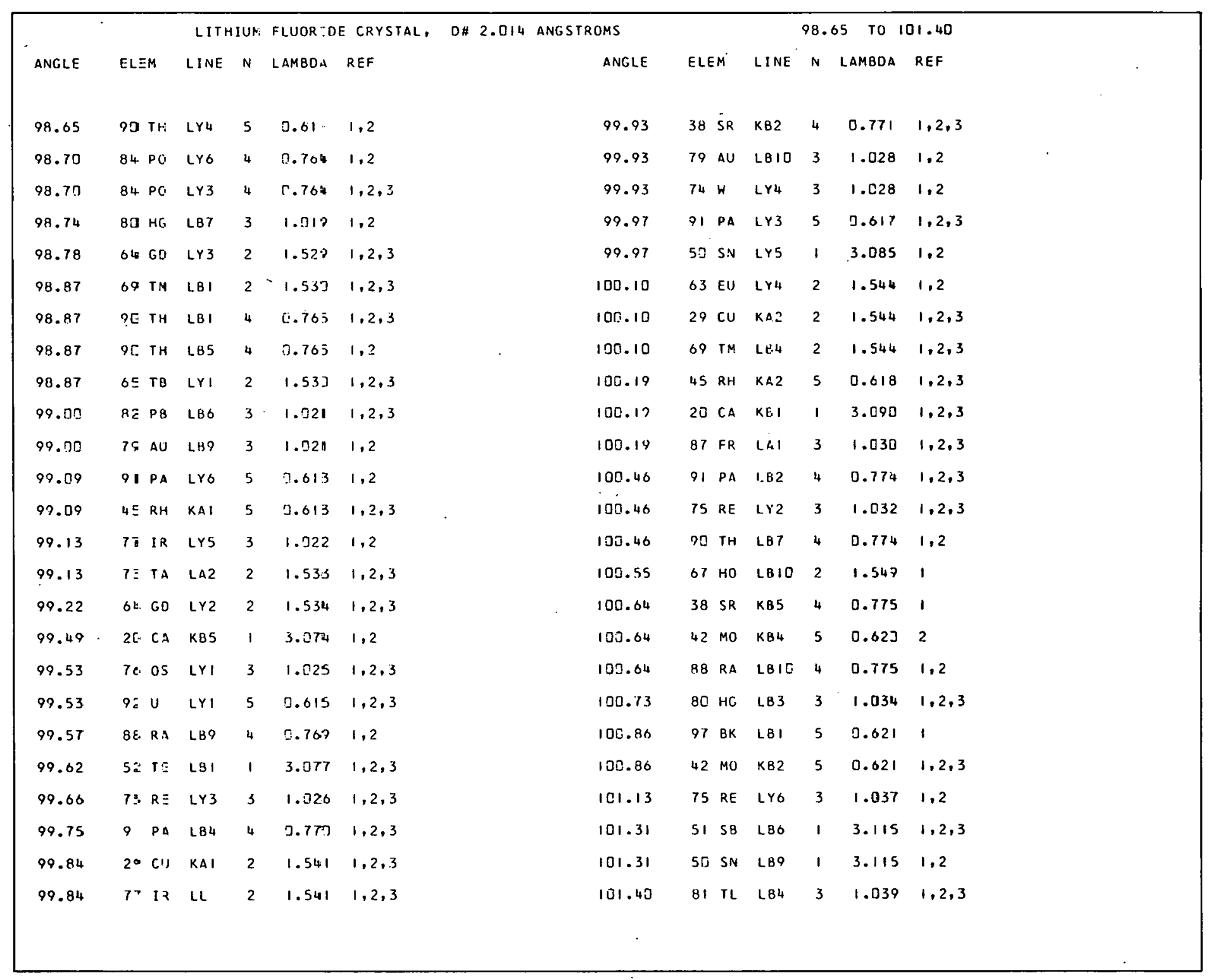




\begin{tabular}{|c|c|c|c|c|c|c|c|c|c|c|c|c|c|c|}
\hline \multirow[b]{2}{*}{ ANGLE } & \multirow[b]{2}{*}{ ELEM } & \multicolumn{2}{|c|}{ LITHIUM } & \multirow{2}{*}{$\begin{array}{l}\text { FLUOR IOE } \\
\text { LAMBDA }\end{array}$} & \multirow{2}{*}{$\begin{array}{l}\text { E CRYSTAL, } \\
\text { REF }\end{array}$} & \multirow{2}{*}{$0: 2.014$} & \multirow{2}{*}{$\begin{array}{l}\text { ANGSTROMS } \\
\text { ANGLE }\end{array}$} & \multirow{2}{*}{\multicolumn{2}{|c|}{ ELEM }} & \multicolumn{4}{|c|}{101.53 T0 103.99} & \\
\hline & & LINE & $N$ & & & & & & & LINE & N & LAMBDA & REF & \\
\hline 101.53 & $8 D \mathrm{HG}$ & LB2 & 3 & 1.040 & $1,2,3$ & & 102.89 & 81 & TL & LB6 & 3 & 1.050 & $1,2,3$ & \\
\hline 101.53 & $91 \mathrm{PA}$ & LYZ & 5 & 0.624 & $1,2,3$ & & 102.98 & 51 & SB & LB3 & 1 & 3.152 & $1,2,3$ & \\
\hline 101.53 & $79 \mathrm{AU}$ & LBS & 3 & 1.040 & 1,2 & & 102.98 & 921 & u & LB6 & 4 & 0.788 & $1,2,3$ & \\
\hline 101.53 & $35 \mathrm{BR}$ & KAI & 3 & 1.040 & $1,2,3$ & & 103.08 & 65 & TB & LYS & 2 & 1.577 & 1 & \\
\hline 101.58 & $50 \mathrm{SN}$ & L810 & 1 & 3.121 & 1,2 & & 103.08 & 65 & TB & LB5 & 2 & 1.577 & 2 & \\
\hline 101.62 & $68 \mathrm{ER}$ & LB3 & 2 & 1.561 & $1,2,3$ & & 103.12 & 50 & SN & $\angle B 7$ & 1 & 3.155 & 1,2 & \\
\hline 101.80 & $87 \mathrm{FR}$ & LA2 & 3 & 1.042 & 1 & & 103.17 & 83 & BI & LY3 & 4 & 0.789 & $1,2,3$ & \\
\hline 102.07 & $35 B R$ & KA2 & 3 & 1.044 & $1,2,3$ & & 103.17 & 89 & $A C$ & LBI & 4 & 0.789 & 1 & \\
\hline 102.07 & $38 \mathrm{SR}$ & KBI & 4 & 0.783 & $1,2,3$ & & 103.26 & 53 & I & LA2 & 1 & 3.158 & $1,2,3$ & \\
\hline 102.07 & $38 \mathrm{SR}$ & KB3 & 4 & 0.783 & 1,2 & & 103.35 & 40 & $2 R$ & KA2 & 4 & 0.790 & $1,2,3$ & \\
\hline 102.16 & $67 \mathrm{HO}$ & LB2 & 2 & 1.567 & $1,2,3$ & & 103.35 & 721 & HF & LA2 & 2 & 1.580 & $1,2,3$ & \\
\hline 102.16 & $O B E R$ & LBG & 2 & 1.567 & $1,2,3$ & & 103.35 & 42 & Mo & KBI & 5 & 0.632 & $1,2,3$ & \\
\hline 102.21 & 42 MO & KBS & 5 & 0.627 & 1 & & 103.35 & 90 & TH & LYO & 5 & 0.632 & 1,2 & \\
\hline 102.21 & 33 AS & $K B 2$ & 3 & 1.045 & $1,2,3$ & & 103.35 & 83 & BI & LYo & 4 & 0.790 & 1,2 & \\
\hline 102.21 & $56 \mathrm{BA}$ & LL & 1 & 3.135 & $1,2,3$ & & 103.44 & 49 & IN & $|Y|$ & 1 & 3.162 & $1,2,3$ & \\
\hline 102.30 & $48 C D$ & LY3 & 1 & 3.137 & 1,2 & & 103.44 & 78 & PT & LBQ & 3 & 1.054 & 1,2 & \\
\hline 102.35 & $72 \mathrm{HF}$ & LAI & 2 & 1.567 & $1,2,3$ & & 103.58 & 42 & Mo & $K B \cdot 3$ & 5 & 0.633 & 1,2 & \\
\hline 102.35 & $48 C D$ & LY2 & 1 & 3.138 & $1,2,3$ & & 103.72 & 98 & CF & LA & 4 & 0.792 & 1 & \\
\hline 102.62 & $402 R$ & KAI & 4 & 0.786 & $1,2,3$ & & 103.81 & 76 & os & LL & 2 & 1.585 & $1,2,3$ & \\
\hline 102.62 & $82 \mathrm{~PB}$ & LY4 & 4 & 0.786 & 1 & & 103.81 & 915 & PA & LYI & 5 & 0.634 & $1,2,3$ & \\
\hline 102.76 & 33 AS & $K 85$ & 3 & 1.049 & 1,2 & & 103.86 & 33 & AS & KBI & 3 & 1.057 & $1,2,3$ & \\
\hline 102.76 & $85 \mathrm{HG}$ & L81 & 3 & 1.049 & $1,2,3$ & & 103.86 & 76 & os & LYS & 3 & 1.057 & 1,2 & \\
\hline 102.82 & $53 \mathrm{I}$ & LA I & 1 & 3.148 & $1,2,3$ & & 103.86 & 86 & RN & LAI & 3 & 1.057 & 1 & \\
\hline 102.80 & $84 P O$ & LYI & 4 & 0.787 & $1,2,3$ & & 103.90 & 90 & TH & L84 & 4 & 0.793 & $1,2,3$ & \\
\hline 102.89 & 79 AU & Lㅂ7 & 3 & 1.050 & 1,2 & & 103.99 & 33 & AS & KB 3 & 3 & 1.058 & 1.2 & \\
\hline
\end{tabular}




\begin{tabular}{|c|c|c|c|c|c|c|c|c|c|c|c|c|c|}
\hline & & LITH & IUM & FLUORIDE & E CRYSTAL, & D\# 2.014 & ANSSTROMS & & & & 03. & 99 ro 1 & 07.53 \\
\hline ANGLE & ELEM & LINE & $N$ & LAMBCA & REF & & ANGLE & ELE & & LINE & $N$ & LAMBCAA & REF \\
\hline 103.99 & $68 \mathrm{ER}$ & $L B \mid$ & 2 & 1.58 .7 & $1,2,3$ & & 105.77 & 88 & RA & LB3 & 4 & $0.3 \mathrm{C} 3$ & 1.2 .3 \\
\hline 104.04 & 50 SN & $\angle B \bar{C}$ & 1 & 3.175 & $1,2,3$ & & 105.77 & 62 & SM & $\mathrm{LY}_{4}$ & 2 & 1.5016 & 1,2 \\
\hline 104.34 & $92 \mathrm{U}$ & LYS & 5 & $0.6 \lesssim 5$ & 1,2 & & 105.86 & 66 & or & LBIO & 2 & 1.60 .7 & 1 \\
\hline 104.05 & $90 \mathrm{TH}$ & $\angle B 2$ & 4 & 0.754 & $1,2,3$ & & 105.91 & 44 & RU & KAI & 5 & 0.643 & $1,2,3$ \\
\hline 104.13 & 83 B I & LN & 3 & 1.059 & $1,2,3$ & & 105.96 & 98 & CF & LA2 & 4 & 0.804 & 1 \\
\hline 104.27 & $9 \mathrm{TH}$ & $\operatorname{LY} 3$ & 5 & 3.636 & $1,2,3$ & & 105.96 & 78 & PT & LBS & 3 & 1.072 & 1,2 \\
\hline 104.36 & $63 \mathrm{EU}$ & LY3 & 2 & 1.501 & $1,2,3$ & & 105.96 & 80 & HG & LB4 & 3 & 1.072 & $1,2,3$ \\
\hline .104 .41 & $7 ذ \mathrm{RE}$ & $|Y|$ & 4 & 1.061 & $1,2,3$ & & 106.05 & 27 & co & KBS & 2 & 1.509 & 1.2 \\
\hline 104.46 & $83 \mathrm{BI}$ & $L Y 2$ & 4 & 0.706 & $1,2,3$ & & 106.15 & 92 & $u$ & LN & 4 & 0.805 & $1,2.3$ \\
\hline 104.40 & $6+60$ & LY: & 2 & 1.502 & $1,2,3$ & & 100.24 & 74 & $w$ & LYO & 3 & 1.074 & 1,2 \\
\hline 104.55 & $74 \mathrm{~W}$ & LY3 & 3 & 1.002 & $1,2,3$ & & 106.34 & 88 & RA & $\therefore 85$ & 4 & 0.806 & 1,2 \\
\hline 104.55 & $73 \mathrm{PT}$ & LBIO & 3 & 1.002 & 1,2 & & 100.43 & 51 & SB & LB I & 1 & 3.226 & $1,2,3$ \\
\hline 104.74 & $51 S B$ & LB4 & 1 & 3.100 & $1,2,3$ & & 156.72 & 91 & PA & LBO & 4 & 0.808 & $1,2,3$ \\
\hline 104.92 & $63 \mathrm{EU}$ & LY2 & 2 & 1.507 & $1,2,3$ & & 106.86 & 44 & Ru & KA2 & 5 & 0.647 & $1,2,3$ \\
\hline $104.9 ?$ & St CM & LBI & 5 & 0.639 & 1 & & 107.00 & 71 & LU & LAI & 2 & 1.619 & $1,2,3$ \\
\hline 104.97 & $73 \mathrm{TA}$ & LY4 & 3 & 1.055 & 1,2 & & 107.00 & 67 & HO & LB3 & 2 & 1.619 & $1,2,3$ \\
\hline 105.11 & $66 \mathrm{Dr}$ & LB7 & 2 & 1.579 & 1,2 & & 107.10 & 80 & HG & LBG & 3 & 1.080 & $1,2,3$ \\
\hline $105.2 n$ & 66 or & LBO & 2 & סרב & 1 & & 107.10 & 97 & $B K$ & LAI & 4 & 0.810 & 1 \\
\hline 105.25 & $92 \mathrm{U}$ & $\mathrm{LL}$ & 3 & 1.057 & 2,3 & & 107.19 & 27 & co & K.BI & 2 & 1.621 & $1,2,3$ \\
\hline 105.39 & $68 \mathrm{ER}$ & LB4 & 2 & 1.631 & $1,2,3$ & & 107.29 & 67 & HO & LB6 & 2 & 1.622 & $1,2,3$ \\
\hline 105.37 & $.4 \mathrm{~W}$ & LY2 & 3 & 1.058 & $1,2,3$ & & 107.34 & 88 & $R A$ & $\mathrm{LY}_{4}$ & 5 & 0.649 & 1,2 \\
\hline 105.37 & TQ AU & LB3 & 3 & 1.058 & $1,2,3$ & & 107.39 & 66 & DY & $\angle B 2$ & 2 & 1.623 & $1,2,3$ \\
\hline 195.53 & $86 \mathrm{RN}$ & LA2 & 3 & 1.058 & 1 & & 107.37 & 78 & PT & LB7 & 3 & 1.092 & 1,2 \\
\hline 105.67 & $79 \mathrm{AU}$ & LB2 & 3 & $1.07 \mathrm{j}$ & $1,2,3$ & & 107.48 & 81 & TL & LY4 & 4 & 0.812 & 1,2 \\
\hline 105.67 & PO TH & LY2 & 5 & 0.642 & $1,2,3$ & & 107.53 & 49 & IN & LYS & 1 & 3.249 & 1,2 \\
\hline
\end{tabular}




\begin{tabular}{|c|c|c|c|c|c|c|c|c|c|c|c|c|c|c|}
\hline \multirow[b]{2}{*}{ ANGLE } & \multirow[b]{2}{*}{ ELEM } & \multicolumn{2}{|c|}{ LITHIUM } & \multirow{2}{*}{$\begin{array}{l}\text { FLUORIDE } \\
\text { LAMBDA }\end{array}$} & \multirow{2}{*}{$\begin{array}{l}\text { E CRYSTAL, } \\
\text { REF }\end{array}$} & \multirow{2}{*}{ D\#2.014 } & \multirow{2}{*}{$\begin{array}{l}\text { ANGSTROMS } \\
\text { ANGLE }\end{array}$} & \multicolumn{6}{|c|}{$107.67 \quad 10 \quad 110.82$} & \\
\hline & & LINE & $N$ & & & & & ELE & & LINE & $\mathbf{N}$ & LAMBDA & REF & \\
\hline 107.67 & $79 \mathrm{AU}$ & $\angle B:$ & 3 & 1.084 & $1,2,3$ & & 109.13 & 84 & GD & LYS & 2 & 1.641 & 1 & \\
\hline 107.67 & $83 \mathrm{BI}$ & $L Y \mid$ & 4 & 0.813 & $1,2,3$ & & 109.23 & 82 & PB & LY2 & 4 & 0.821 & $1,2,3$ & \\
\hline 107.82 & BS AT & LAI & 3 & 1.085 & 1 & & 109.43 & 97 & $8 K$ & LA2 & 4 & 0.822 & 1 & \\
\hline 107.87 & $89 \mathrm{AC}$ & L82 & 4 & 0.814 & 1 & & 109.43 & 37 & RB & KB5 & 4 & 0.822 & 1 & \\
\hline 167.87 & $88 R A$ & L81 & 4 & 0.814 & $1,2,3$ & & 109.48 & 52 & TE & LAI & $!$ & 3.289 & $1,2,3$ & \\
\hline 199.06 & $71 \mathrm{LU}$ & LA2 & 2 & 1.630 & $1,2,3$ & & 109.53 & 95 & $A M$ & LBI & 5 & 0.658 & 1,3 & \\
\hline 108.06 & $75 \mathrm{RE}$ & LL & 2 & 1.630 & $1,2,3$ & & 109.58 & 77 & IR & L810 & 3 & 1.097 & 1,2 & \\
\hline 108.06 & $82 \mathrm{~PB}$ & $\operatorname{LY} 3$ & 4 & 0.815 & $1,2.3$ & & 109.58 & 85 & $A T$ & LA2 & 3 & 1.097 & 1 & \\
\hline 108.26 & 88 RA & LB 7 & 4 & 0.816 & 1,2 & & 109.73 & 67 & HO & LBI & 2 & 1.647 & $1,2,3$ & \\
\hline 108.26 & 37 RB & KB2 & 4 & 0.816 & $1,2,3$ & & 109.87 & 73 & TA & LY3. & 3 & 1.099 & $1,2,3$ & \\
\hline 108.30 & $98 \mathrm{CF}$ & LB2 & 5 & 0.653 & 1 & & 109.87 & 74 & iw & LYYI & 3 & 1.099 & $1,2,3$ & \\
\hline 108.30 & $90 \mathrm{TH}$ & LYI & 5 & 0.653 & $1,2,3$ & & 109.92 & 52 & TE & LA2 & 1 & 3.298 & $1,2,3$ & \\
\hline 108.45 & $55 \mathrm{CS}$ & LL & 1 & 3.267 & $1,2,3$ & & 110.02 & 41 & NB & KB5 & 5 & 0.660 & 1 & \\
\hline 108.45 & $82 \mathrm{~PB}$ & LYG & 4 & 0.817 & 1,2 & & 110.32 & 78 & PT & LB2 & 3 & 1.102 & $1,2,3$ & \\
\hline 108.45 & $49 \mathrm{lin}$ & LBQ & 1 & 3.268 & 1,2 & & 110.32 & 47 & $A G$ & LY2 & 1 & 3.306 & 1,3 & \\
\hline 108.50 & $50 \mathrm{SNN}$ & LB6 & 1 & 3.269 & $1,2,3$ & & $110.32^{\circ}$ & 50 & SN & LB3 & 1 & 3.306 & $1,2,3$ & \\
\hline 108.55 & $41 \mathrm{NB}$ & $K B 2$ & 5 & 0.654 & $1,2,3$ & & 110.32 & 47 & $A G$ & LY3 & 1 & 3.306 & 1.2 & \\
\hline 108.55 & $77 \mathrm{IR}$ & LBO & 3 & 1.090 & 1,2 & & 110.47 & 72 & $\mathrm{HF}$ & $\mathrm{LY}_{4}$ & 3 & 1.103 & 1,2 & \\
\hline 108.64 & $70 Y B$ & Liv & 2 & 1.036 & $1,2,3$ & & 110.52 & 62 & $S M$ & LY3 & 2 & 1.655 & $1,2,3$ & \\
\hline 108.69 & $Q 1 P A$ & LL & 3 & 1.091 & $1,2,3$ & & 110.62 & 78 & PT & LB3 & 3 & 1.104 & $1,2,3$ & \\
\hline 108.74 & $49 \mathrm{IN}$ & LBIO & 1 & 3.274 & 1,2 & & 110.62 & 90 & $\mathrm{TH}$ & LBO & 4 & 0.828 & $1,2,3$ & \\
\hline 108.79 & $91 \mathrm{PA}$ & LYS & 5 & 0.655 & 1,2 & & 110.77 & 34 & SE & KAI & 3 & 1.105 & $1,2,3$ & \\
\hline 108.84 & $82 \mathrm{Pl}$ & LN & 3 & 1.092 & $1,2,3$ & & 110.77 & 73 & TA & LY2 & 3 & 1.105 & $1,2,3$ & \\
\hline 109.04 & $53 \mathrm{I}$ & LN & 1 & 3.280 & $1,2,3$ & & 110.82 & 96 & $C M$ & LAI & 4 & 0.829 & 1 & \\
\hline 109.13 & $75 R E$ & LYS & 3 & 1.094 & 1,2 & & 110.82 & 39 & $r$ & KAI & 4 & 0.829 & $1,2,3$ & \\
\hline & & & & & & & & & & & & & $\cdot$ & t \\
\hline
\end{tabular}




\begin{tabular}{|c|c|c|c|c|c|c|c|c|c|c|c|c|c|c|}
\hline \multirow[b]{2}{*}{ ANGLE: } & \multirow[b]{2}{*}{ ELEM } & \multicolumn{2}{|c|}{ LITHIUY } & \multirow{2}{*}{\multicolumn{2}{|c|}{$\begin{array}{l}\text { FLUORIOE CRYSTAL, } \\
\text { LAMBCA REF }\end{array}$}} & \multirow{2}{*}{\multicolumn{2}{|c|}{ D\# 2.014}} & \multirow{2}{*}{$\begin{array}{l}\text { ANGSTROMS } \\
\text { ANGLE }\end{array}$} & \multirow[b]{2}{*}{ ELEM } & \multicolumn{4}{|c|}{.$\quad 110.82$ to 112.29} & \\
\hline & & LINE & $\mathrm{N}$ & & & & & & & LINE & $\mathbf{N}$ & LAMBDA & REF & \\
\hline 115.82 & $91 P A$ & L.N & 4 & 0.829 & $1,2,3$ & & & 112.29 & $97 \mathrm{BK}$ & L32 & 5 & 0.869 & 1 & \\
\hline & $63 \mathrm{EJ}$ & LYI & 2 & 1.658 & $1,2,3$ & & & 112.29 & $90 \mathrm{TH}$ & LL & 3 & 1.115 & $1,2,3$ & - \\
\hline 110.82 & $37 \mathrm{RB}$ & $K B ?$ & 4 & $0.8 \leq 9$ & 1,2 & & & & & & & & & \\
\hline 110.82 & $3 i R B$ & KBI & 4 & $3.8 \div 9$ & $1,2,3$ & & & & & & & & & \\
\hline 110.82 & $28 \mathrm{NI}$ & KAI & 2 & 1.858 & $1,2,3$ & & & & & & & & & \\
\hline 110.92 & 70 IR & LB5 & 3 & 1.106 & 1,2 & & & & & & & & & \\
\hline $110.9 \hat{c}$ & $62 \mathrm{SM}$ & LYż & 2 & 1.659 & $1,2,3$ & & & & & & & & & \\
\hline $110.9 \bar{c}$ & $65 \mathrm{TH}$ & LB7 & 2 & 1.659 & 1,2 & & & & & & & & & \\
\hline $110.9 \bar{c}$ & $67 \mathrm{HO}$ & LBL & 2 & 1.659 & $1,2,3$ & & & & & & & & & \\
\hline 111.07 & $78 \mathrm{AU}$ & $\mathrm{LB} 4$ & 3 & 1.187 & $1,2,3$ & & & & & & & & & \\
\hline $111.2 \%$ & $23 \mathrm{NI}$ & $K A 2$ & 2 & 1.602 & $1,2,3$ & & & & & & & & & \\
\hline הל.1ו1 & $3+S \bar{E}$ & $K A 2$ & 3 & 1.109 & $1,2,3$ & & & & & & & & & \\
\hline 111.52 & $41 \mathrm{NB}$ & KB3. & 5 & 0.606 & 1,2 & & & & & & & $\cdot$ & & \\
\hline 111.52 & $41 N \mathrm{NB}$ & $K 81$ & 5 & 9.006 & $1,2,3$ & & & & & & & & & \\
\hline 111.63 & $37 \mathrm{Y}$ & KA2 & L & 0.833 & $1,2, \overline{3}$ & & & & & . & & & . & \\
\hline 111.68 & $79 \mathrm{AU}$ & LBó & 3 & 1.111 & $1,2,3$ & & & & & & & & & \\
\hline 111.73 & 6.5 I B & LBIO & 2 & 1.637 & 1,2 & & . & ' & & & & & & \\
\hline 111.83 & $48 C 0$ & LYI & 1 & 3.336 & $1,2,3$ & & & & & & & & & \\
\hline 111.93 & 49 IN & LB 2 & 1 & 3.338 & $1,2,3$ & & & & & & & & & \\
\hline 112.53 & $88 \mathrm{KA}$ & LB2 & 4 & 0.835 & $1,2,3$ & & & & & & & & & \\
\hline 112.13 & $13 \mathrm{TA}$ & LYS & 3 & 1.1114 & 1,2 & & & & & & & & & \\
\hline 112.13 & $84 P O$ & LAI & 3 & 1.114 & $1,2,3$ & & & & & & & & & \\
\hline 112.13 & $5 \mathrm{SN}$ & LB4 & 1 & 3.343 & $1,2,3$ & & & & & & & & & . \\
\hline 112.24 & $T C Y B$ & LAI & 2 & 1.672 & $1,2,3$ & & & & & & & & & \\
\hline 112.27 & $Y 7 I R$ & LB7 & 3 & 1.115 & 1,2 & & & & & & & & & \\
\hline
\end{tabular}




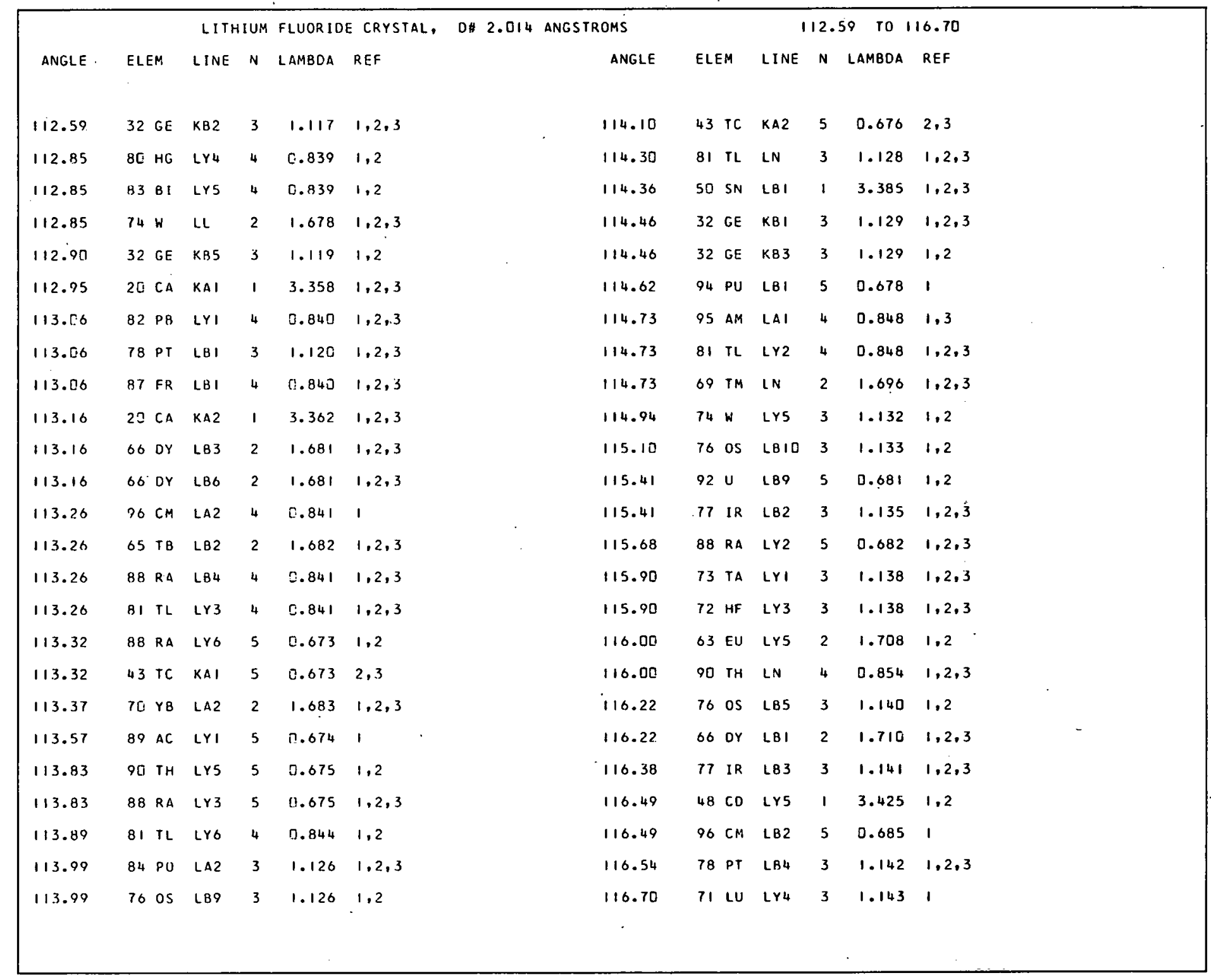




\begin{tabular}{|c|c|c|c|c|c|c|c|c|c|c|c|c|c|c|}
\hline \multirow[b]{2}{*}{ ANGLE } & \multirow[b]{2}{*}{ ELEM } & \multicolumn{2}{|c|}{ LITHIUM } & \multirow{2}{*}{$\begin{array}{l}\text { FLUORIDE } \\
\text { LAMBOA }\end{array}$} & \multirow{2}{*}{$\begin{array}{l}\text { E CRYSIAL, } \\
\text { REF }\end{array}$} & \multirow{2}{*}{$D * 2.014$} & \multirow{2}{*}{$\begin{array}{l}\text { ANGSTROMS } \\
\text { ANGLE }\end{array}$} & \multirow{2}{*}{\multicolumn{2}{|c|}{ ELEM }} & \multicolumn{4}{|c|}{$116.76 \quad$ ro 120.38} & \\
\hline & & LINE & N. & & & & & & & LINE & $N$ & LAMBDA & REF & \\
\hline 116.76 & $48 \mathrm{CD}$ & LB9 & 1 & 3.430 & 1,2 & & 118.63 & $36 r$ & KR & KB2 & 4 & 0.806 & $1,2,3$ & \\
\hline 116.87 & $87 \mathrm{FR}$ & LB2 & 4 & 0.858 & $1,2,3$ & & 118.63 & 82 & PB & LYS & 4 & 0.856 & 1,2 & \\
\hline 116.87 & 8381 & LA! & 3 & 1.144 & $1,2,3$ & & 118.68 & 83 & $B I$ & LA2 & 3 & 1.155 & $1,2,3$ & \\
\hline 116.87 & $78 \mathrm{PT}$ & LB6 & 3 & 1.144 & $1,2,3$ & & 118.68 & 72 & HF & LYo & 3 & 1.155 & 1.2 & \\
\hline 116.87 & $72 \mathrm{MF}$ & LY2 & 3 & 1.144 & $1,2,3$ & & 118.96 & 49 & IN & LB3 & 1 & 3.470 & $1,2,3$ & \\
\hline 117.03 & 920 & 1810 & 5 & $0.68 \mathrm{r}$ & 1,2 & & 119.08 & 81 & $\mathrm{TL}$ & LYI & 4 & 0.868 & $1,2,3$ & \\
\hline 117.08 & 49 IN & LB6 & 1 & 3.435 & $1,2,3$ & & 119.08 & 94 & PU & $|A|$ & 4 & 0.868 & 1 & \\
\hline 117.14 & $48 \mathrm{CD}$ & LBIJ & 1 & 3.437 & 1,2 & & 119.19 & 77 & IR & $L B I$ & 3 & 1.158 & $1,2,3$ & \\
\hline 117.19 & $52 \mathrm{TE}$ & LN & 1 & 3.433 & $1,2,3$ & & 119.19 & 69. & $T M$ & LA2 & 2 & 1.737 & $1,2,3$ & \\
\hline 117.25 & $515 B$ & $|A|$ & 1 & 3.437 & $1,2,3$ & & 119.24 & 88 & RA & LYI & 5 & 0.695 & $1,2,3$ & \\
\hline 117.30 & $95 \mathrm{AM}$ & LA2 & 4 & 0.867 & 1,3 & & 119.30 & 80 & HG & $\operatorname{lr} 3$ & 4 & 0.869 & $1,2,3$ & \\
\hline 117.30 & 66 OY & LBH & 2 & 1.723 & $1,2,3$ & & 119.53 & 40 & $2 R$ & K65 & 5 & 0.698 & 1 & \\
\hline 117.36 & $10 \mathrm{~K}$ & KB5 & 1 & 3.441 & 1,2 & & 119.64 & 65 & TB & 186 & 2 & 1.741 & $1,2,3$ & \\
\hline 117.63 & $64 \mathrm{GD}$ & $\angle B 7$ & 2 & 1.723 & 1,2 & & 119.75 & 361 & $K R$ & KB5 & 4 & 0.871 & 1.2 & . \\
\hline 117.69 & 76 os & LB 7 & 3 & 1.149 & 1,2 & & 119.75 & 88 & $R A$ & 186 & 4 & 0.871 & $1,2,3$ & \\
\hline 117.74 & 6460 & LB9 & 2 & 1.724 & 1 & $\cdot$ & 119.98 & 26 & $\mathrm{FE}$ & KB5 & 2 & 1.744 & 1,2 & \\
\hline 117.74 & 5158 & LA2 & 1 & 3.448 & $1,2,3$ & & 119.98 & 601 & NO & LY4 & 2 & 1.744 & 1,2 & \\
\hline 117.85 & $402 R$ & KB2 & 5 & 0.690 & $1,2,3$ & & 120.04 & 46 & PD & LY2 & 1 & 3.489 & $1,2,3$ & \\
\hline 117.96 & $67 \mathrm{IM}$ & LAI & 2 & 1.726 & $1,2,3$ & & 120.04 & 46 & PD & $\operatorname{Lr} 3$ & 1 & 3.489 & 1,2 & \\
\hline 118.07 & 625.4 & LYI & 2 & 1.727 & $1,2,3$ & & 120.09 & 931 & NP & LBI & 5 & 0.098 & $1.2,3$ & \\
\hline 118.97 & $19 \mathrm{~K}$ & KBI & 1 & $3.4 \leq 4$ & $1,2,3$ & & 120.09 & 64 & GD & LB2 & 2 & 1.745 & 1.2 .3 & \\
\hline 118.18 & $73 \mathrm{TA}$ & $\mathbf{L L}$ & 2 & 1.728 & $1,2,3$ & & 120.21 & 65 & $T B$ & LB3 & 2. & 1.746 & $1,2,3$ & \\
\hline 118.52 & $64 \mathrm{GD}$ & LBID & 2 & $1.7 \geq 1$ & 1,2 & & 120.21 & 80 & HG & $\operatorname{LN}$ & 3 & 1.164 & $1,2,3$ & \\
\hline 118.63 & $86 \mathrm{RN}$ & LBI & 4 & 0.86 .6 & 1 & & 120.21 & 80 & HG & LYG & 4 & 0.873 & 1,2 & \\
\hline 118.63 & $36 \mathrm{KR}$ & KB 4 & 4 & 0.866 & 2 & & 120.38 & 75 & $R E$ & LBQ & 3 & 1.165 & 1,2 & \\
\hline
\end{tabular}




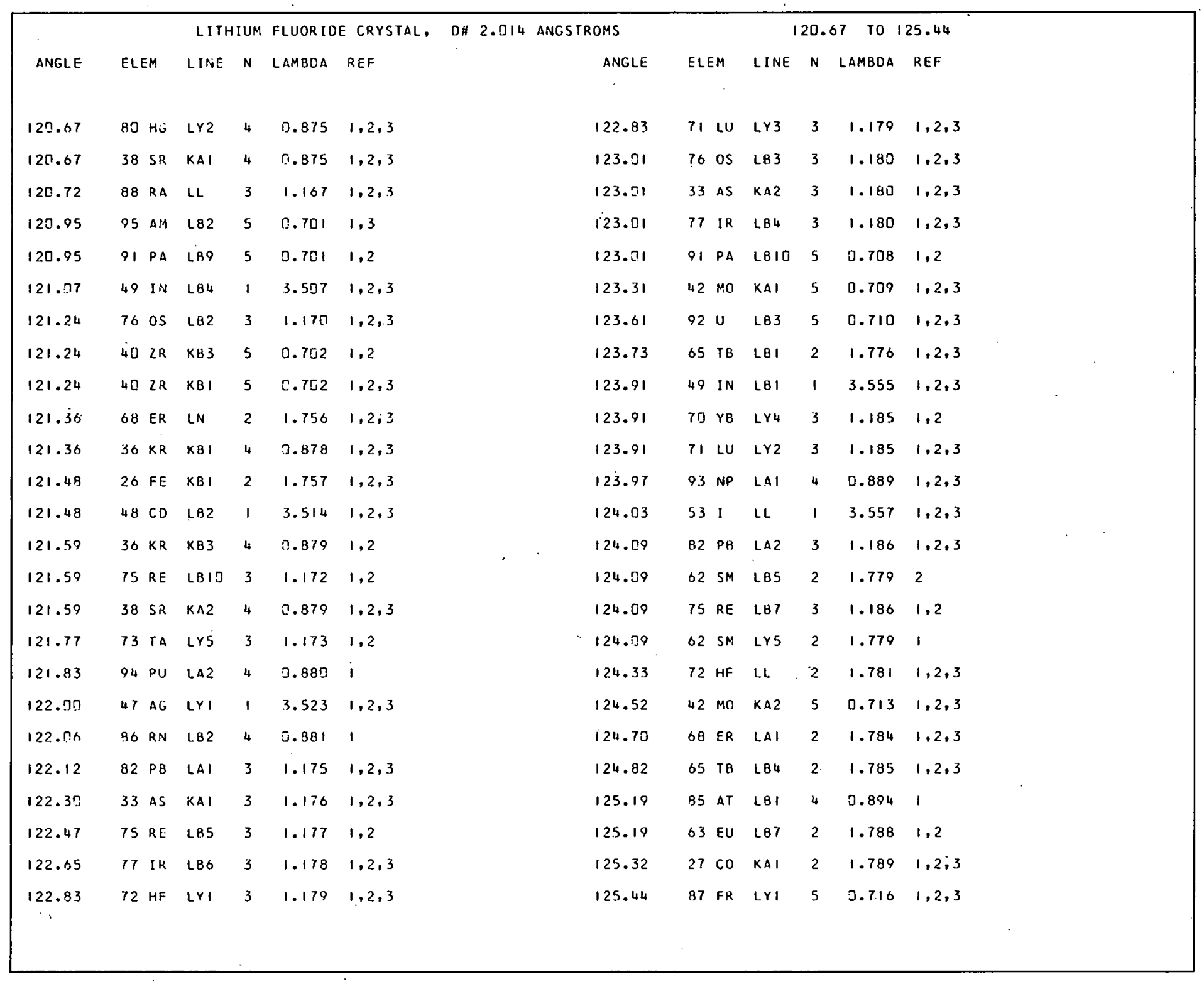




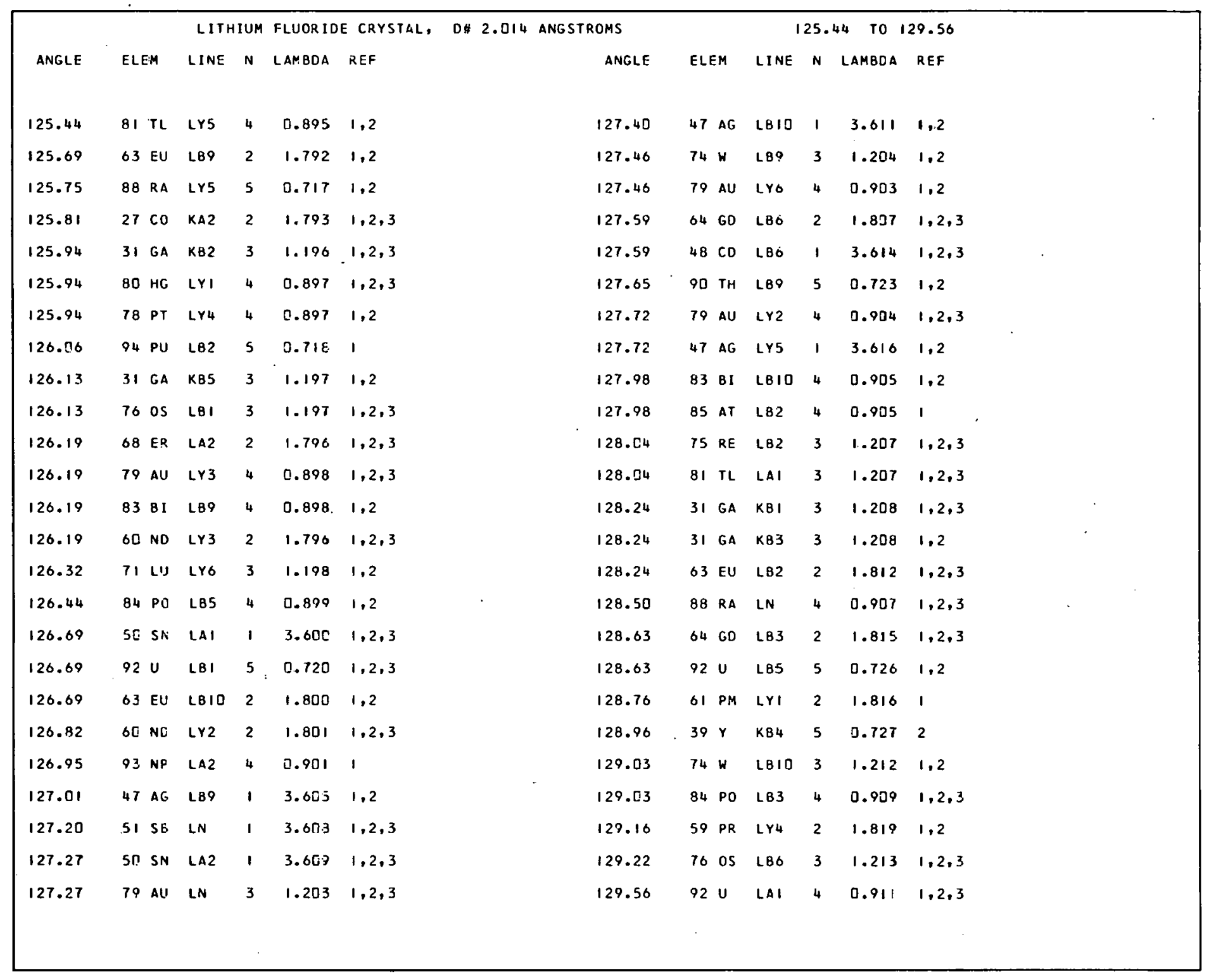




\begin{tabular}{|c|c|c|c|c|c|c|c|c|c|c|c|c|}
\hline & & LITH & IUM & FLUOK IOE & E CRYSTAL, & $0 \# 2.014$ & ANGSTROMS & & & 29. & TO 13 & 34.90 \\
\hline ANGLE & ELEM & LINE & $N$ & LAMBDA & REF & & ANGLE & ELEM & LINE & $N$ & LAMBDA & REF \\
\hline 129.62 & $48 \mathrm{Cl}$ & LB3 & 1 & 3.645 & $1,2,3$ & & 132.72 & $67 \mathrm{HO}$ & LAI & 2 & 1.845 & $1,2,3$ \\
\hline 129.62 & $72 \mathrm{HF}$ & LYS & 3 & 1.215 & 1,2 & & 132.86 & $64 \mathrm{GD}$ & LBI & 2 . & 1.846 & $1,2,3$ \\
\hline 129.62 & $39 \mathrm{Y}$ & KB2 & 5 & 0.729 & $1,2,3$ & & 133.08 & $86 \mathrm{RN}$ & $\lfloor Y)$ & 5 & 0.739 & 1 \\
\hline 129.62 & $74 \mathrm{~W}$ & LBS & 3 & 1.215 & 1,2 & & 133.15 & 80 HG & LYS & 4 & 0.924 & 1,2 \\
\hline 129.96 & $9[\mathrm{TH}$ & LAIC & 5 & 0.730 & 1,2 & & 133.44 & $35 \mathrm{BR}$ & KB5 & 4 & 0.925 & 1,2 \\
\hline 130.09 & $67 \mathrm{HO}$ & LN & 2 & 1.826 & $1,2,3$ & & 133.65 & $47 A G$ & L82 & 1 & 3.703 & $1,2,3$ \\
\hline 130.23 & 7605 & LA4 & 3 & 1.218 & $1,2,3$ & & 133.72 & $83 B 1$ & LB5 & 4 & 0.926 & 1,2 \\
\hline 130.43 & $81 \mathrm{TL}$ & LA2 & 3 & 1.21 .9 & $1,2,3$ & & 1.33 .72 & $37 R B$ & $\mathrm{KAI}$ & 4 & 0.926 & $1,2,3$ \\
\hline 130.64 & $91 \mathrm{PA}$ & L83 & 5 & {$[1.732$} & $1,2,3$ & & 133.80 & $39 \mathrm{Y}$ & KaI & 5 & 0.741 & $1,2,3$ \\
\hline 130.64 & $75 R E$ & LB3 & 3 & 1.220 & $1,2,3$ & & 133.80 & $39 \mathrm{r}$ & KB3 & 5 & 0.741 & 1,2 \\
\hline 131.05 & $71 \mathrm{L1}$ & LYI & 3 & 1.222 & $1,2,3$ & & 133.87 & $64 \mathrm{GO}$ & LB4 & 2 & 1.853 & $1,2,3$ \\
\hline $131 . .5$ & $70 Y B$ & LY3 & 3 & 1.222 & $1,2,3$ & & 134.91 & 77 IR & LY4 & 4 & 0.927 & 1,2 \\
\hline 131.32 & $39 \mathrm{Y}$ & KB5 & 5 & 0.734 & 1 & & 134.81 & $79 \mathrm{AU}$ & LYI & 4 & 0.927 & $1,2,3$ \\
\hline 131.46 & $74 \mathrm{~W}$ & L87 & 3 & 1.224 & 1,2 & & 134.01 & 82 PB & L89 & 4 & 0.927 & 1,2 \\
\hline 131.46 & $71 \mathrm{LU}$ & LL & 2 & 1.836 & $1,2,3$ & & 134.16 & $60 \mathrm{ND}$ & LYo & 2 & 1.855 & 2 \\
\hline 132.02 & $92 \mathrm{U}$ & LB7 & 5 & 0.736 & 1.2 & & 134.16 & 91 PA & LBI & 5 & 0.742 & $1,2,3$ \\
\hline 132.02 & $93 \mathrm{NP}$ & LB2 & 5 & 0.736 & $1,2,3$ & & 134.31 & $62 S M$ & L87 & 2 & 1.856 & 1,2 \\
\hline 132.16 & $48 C D$ & LB4 & 1 & 3.682 & $1,2,3$ & & 134.31 & $67 \mathrm{HO}$ & LA2 & 2 & 1.856 & $1,2,3$ \\
\hline 132.37 & $35 B R$ & K82 & 4 & 0.921 & 1.2 .3 & & 134.31 & $78 \mathrm{PT}$ & LY3 & 4 & 0.928 & $1,2,3$ \\
\hline 132.37 & $45 \mathrm{RH}$ & LYj & 1 & 3.685 & 1,2 & & 134.60 & $84 P 0$ & L82 & 4 & 0.929 & $1,2,3$ \\
\hline 132.37 & $45 \mathrm{RH}$ & LY2 & 1 & 3.685 & $1,2,3$ & & 134.67 & $75 \mathrm{RE}$ & $\lfloor B \mid$ & 3 & 1.239 & $1,2,3$ \\
\hline $132: 51$ & $70 \mathrm{Yu}$ & LY2 & 3 & 1.229 & $1,2,3$ & & 134.67 & $52 \mathrm{TE}$ & LL & 1 & 3.717 & $1,2,3$ \\
\hline 132.51 & $69 \mathrm{TM}$ & LYu & 3 & 1.229 & 1 & & 134.90 & $37 \mathrm{RB}$ & KAZ & 4 & 0.930 & $1,2,3$ \\
\hline 132.58 & $84 P 0$ & LBI & 4 & 0.922 & $1,2,3$ & & & & & & & \\
\hline 132.58 & $92 \mathrm{U}$ & LA2 & 4 & 0.922 & $1,2,3$ & & & & & & & \\
\hline
\end{tabular}




\begin{tabular}{|c|c|c|c|c|c|c|c|c|c|c|c|c|c|c|c|c|}
\hline & & LITH & IUM & FLUOR IDE & E CRYSTAL, & D\# 2.014 & ANGSTROMS & & & & 35. & I0 1 & 40.75 & & & \\
\hline ANGLE & ELEM & LINE & $\mathrm{N}$ & LAMBDA & REF & & ANGLE & ELE & $E M$ & LINE & N & LAMBDA & REF & & & \\
\hline 135.05 & $62 \mathrm{SM}$ & LB9 & 2 & 1.861 & 1.2 & & 137.65 & 601 & NO & LYI & 2 & 1.878 & $1,2.3$ & & & . \\
\hline 135.12 & $8 \mathrm{U} H G$ & LAI & 3 & 1.241 & $1,2, .3$ & & 137.65 & 83 & BI & LB3 & 4 & 0.939 & $1,2,3$ & & & \\
\hline 135.19 & $46 P D$ & LYI & 1 & 3.72 .4 & $1,2,3$ & & 137.80 & 59 & PR & LY2 & 2 & 1.879 & $1,2,3$ & & & \\
\hline 135.27 & $91 \mathrm{PA}$ & LB5 & 5 & c. 745 & 1,2 & & 137.88 & 80 & HG & LAL & 3 & 1.253 & $1,2.3$ & & & \\
\hline 135.57 & $70 Y B$ & LY6 & 3 & 1.243 & 1,2 & & 138.12 & 73 & $T A$ & 1810 & 3 & 1.254 & 1.2 & . & & \\
\hline 135.57 & $78 \mathrm{PI}$ & LN & 3 & 1.243 & $1,2,3$ & & 138.12 & 32 & GE & KAI & 3 & 1.254 & $1,2,3$ & & & \\
\hline 135.64 & $41 N 3$. & KAI & 5 & 0.746 & $1,2,3$ & & 138.28 & 62 & $S M$ & $\angle B 2$ & 2 & 1.882 & $1,2,3$ & & & \\
\hline 135.80 & 3582 & $K B 3$ & 4 & 0.933 & 1,2 & & 138.36 & 73 & TA & L85 & 3 & 1.255 & 1,2 & & & \\
\hline 135.80 & $35 B R$ & $K B I$ & 4 & $\bar{u} .933$ & $1,2,3$ & & 138.93 & 49 & IN & LAI & 1 & 3.772 & 1,3 & & & \\
\hline 135.80 & $91 P A$ & $|A|$ & 4 & 0.933 & $1,2,3$ & & 139.09 & 32 & GE & $K 42$ & 3 & 1.258 & $1,2,3$ & & & \\
\hline 136.02 & $74 \mathrm{~W}$ & L82 & 3 & 1.245 & $1,2,3$ & & 139.09 & 63 & EU & L33 & 2 & 1.887 & $1,2,3$ & & & \\
\hline 136.10 & $78 \mathrm{PT}$ & $\operatorname{Lr} 2$ & 4 & 0.934 & $1,2,3$ & & 139.17 & 90 & $T H$ & LB3 & 5 & 0.755 & $1,2,3$ & & & \\
\hline 136.15 & $78 \mathrm{PT}$ & LYE. & 4 & $0.9 \equiv 4$ & 1,2 & & 139.17 & 91 & $P A$ & LB7 & 5 & 0.755 & 1,2 & & & \\
\hline 136.15 & $82 \mathrm{~PB}$ & LBIO & 4 & $0.9 \leqslant 4$ & 1,2 & & 139.17 & 92 & $u$ & $\angle B 2$ & 5 & 0.755 & $1,2,3$ & & & \\
\hline 136.25 & $62 \mathrm{SMM}$ & $\angle 810$ & 2 & 1.88 .9 & 1,2 & & 139.33 & 75 & RE & LB 4 & 3 & 1.259 & $1,2,3$ & & & \\
\hline 136.25 & $73 \mathrm{TA}$ & LBS & 3 & 1.21 .6 & 1,2 & & 139.58 & 91 & $P A$ & LA2 & 4 & 0.945 & $1,2,3$ & & & \\
\hline 136.25 & $48 \mathrm{CD}$ & (B) & 1 & $3.73 \mathrm{~d}$ & $1,2,3$ & & 139.58 & 71 & LU & LYS & 3 & 1.260 & 1,2 & & & \\
\hline $136.4 \pi$ & $92 \mathrm{U}$ & $\llcorner 84$ & 5 & $3.7 \leftarrow 8$ & $1,2,3$ & & 139.66 & 49 & IN & LAZ & 1 & 3.781 & $1,2,3$ & $\cdot$ & & \\
\hline $136.4 \pi$ & $83 \mathrm{BI}$ & LB7 & 4 & 3.935 & 1,2 & & 140.24 & 84 & PO & $\mathrm{LBH}_{4}$ & 4 & 0.947 & $1,2,3$ & & & \\
\hline 136.48 & $19 \mathrm{~K}$ & KAI & 1 & 3.741 & $1,2,3$ & & 140.24 & 70 & $Y B$ & LL & 2 & 1.894 & $1,2,3$ & & & \\
\hline 136.79 & $19 \mathrm{~K}$ & KA2 & 1 & 3.745 & $1,2,3$ & & 140.33 & 74 & $w$ & LB3 & 3 & 1.263 & $1,2,3$ & & & \\
\hline 137.02 & $63 \mathrm{EU}$ & $\angle B 6$ & 2 & 1.874 & $1,2,3$ & & 140.33 & 50 & SN & LN & 1 & 3.789 & $1,2,3$ & & & \\
\hline 137.02 & $59 P R$ & LY3 & 2 & $1.8 \div 4$ & $1,2,3$ & & 140.58 & 46 & PD & LBQ & 1 & 3.792 & 1,2 & & & \\
\hline 137.18 & $41 \mathrm{NB}$ & $\mathrm{KA} 2$ & 5 & 0.750 & $1,2,3$ & & 140.58 & 73 & $T A$ & L87 & 3 & 1.264 & 1,2 & & ․ & \\
\hline 137.41 & $75 \mathrm{RE}$ & LBo & 3 & 1.251 & $1,2,3$ & & 140.75 & 66 & OY & LN & 2 & 1.897 & $1,2,3$ & & & \\
\hline
\end{tabular}




\begin{tabular}{|c|c|c|c|c|c|c|c|c|c|c|c|c|c|c|}
\hline & & LITH & IUM & & E CRYSTAL, & $D \# ?$ & 2.014 & ANGSTROMS & & & & 40. & 75 Tо 1 & 47.80 \\
\hline AINGLE & ELEM & LINE & in & LAMBDA & REF & & & ANGLE & ELE & & LINE & $\mathbf{N}$ & LAMBDA & REF \\
\hline 140.75 & $25, \mathrm{MN}$ & KBS & 2 & 1.897 & 1,2 & & & 144.20 & 47 & $A G$ & LB3 & 1 & 3.833 & $1,2,3$ \\
\hline 141.09 & $58 \mathrm{CE}$ & $\mathrm{LY}_{4}$ & 2 & 1.899 & 1,2 & & & 144.48 & 76 & OS & $1 r_{4}$ & 4 & 0.959 & 1,2 \\
\hline 141.17 & $46 P D$ & LAIO & 1 & 3.799 & 1,2 & & & 144.48 & 77 & IR & $6 Y 3$ & 4 & 0.959 & $1,2,3$ \\
\hline 141.60 & $69 \mathrm{TM}$ & LY3 & 3 & 1.268 & $1,2,3$ & & & 144.85 & 63 & EU & LBI & 2 & 1.920 & $1,2,3$ \\
\hline 141.60 & $70 \mathrm{YB}$ & $L Y \mid$ & 3 & 1.268 & $1,2,3$ & & & 144.85 & 66 & DY & LA2 & 2 & 1.920 & $1,2,3$ \\
\hline 141.69 & 8381 & $\mathrm{LY}_{4}$ & 5 & 0.761 & $i, 2$ & & & 145.33 & 88 & RA & LBQ & 5 & 0.769 & 1,2 \\
\hline 141.95 & $83 \mathrm{BI}$ & $\lfloor B \mid$ & 4 & 0.952 & $1,2,3$ & & & 145.42 & 74 & $w$ & LBI & 3 & 1.282 & $1.2,3$ \\
\hline 141.95 & $47 A G$ & 186 & 1 & 3.808 & $1,2,3$ & & & 145.61 & 82 & $P B$ & LB 7 & 4 & 0.962 & 1,2 \\
\hline 142.30 & $82 \mathrm{~PB}$ & LBS & 4 & 5.953 & 1,2 & & & 145.81 & 91 & $P A$ & L84 & 5 & 0.770 & $1,2,3$ \\
\hline 142.57 & $85 \mathrm{AT}$ & $|Y|$ & 5 & 0.763 & 1 & & & 146.00 & 63 & EU & LB 4 & 2 & 1.926 & $1,2,3$ \\
\hline 142.83 & Oo DY & LAI & 2 & 1.909 & $1,2,3$ & & & 146.00 & 77 & IR & LN & 3 & 1.284 & $1,2,3$ \\
\hline 143.01 & $25 \mathrm{MN}$ & KBI & 2 & 1.910 & $1,2,3$ & & & 146.00 & 73 & TA & LB 2 & 3 & 1.284 & $1,2,3$ \\
\hline 143.01 & $84 P_{0}$ & $\operatorname{Lr} 3$ & 5 & 0.704 & $1,2,3$ & & & 146.00 & 30 & $2 N$ & KB2 & 3 & 1.284 & $1,2,3$ \\
\hline 143.01 & 8381 & LB2 & 4 & 0.955 & $1,2,3$ & & & 146.29 & 30 & $2 N$ & K85 & 3 & 1.285 & 1.2 \\
\hline 143.21 & $84 \mathrm{PO}$ & Lro. & 5 & 0.764 & 1,2 & & & 146.29 & 38 & $S R$ & KB2 & 5 & 0.771 & $1,2,3$ \\
\hline 143.19 & $69 \mathrm{TM}$ & $\operatorname{Lr} 2$ & 3 & 1.274 & $1,2,3$ & & & 146.39 & 81 & $\mathrm{TL}$ & 2810 & 4 & 0.964 & 1,2 \\
\hline 143.19 & $46 P D$ & LYS & 1 & 3.822 & 1,2 & & & 146.79 & 77 & IR & LY2 & 4 & 0.965 & $1,2,3$ \\
\hline 143.37 & $90 \mathrm{TH}$ & $\lfloor A \mid$ & 4 & 0.956 & $1,2,3$ & & & 147.19 & 79 & $A U$ & $i_{A 2}$ & 3 & 1.288 & $1,2,3$ \\
\hline 143.37 & 79 All & LY5 & 4 & 0.956 & $.1,2$ & & & 147.59 & 77 & IR & LYo & 4 & 0.967 & 1,2 \\
\hline 143.46 & $90 \mathrm{TH}$ & $|B|$ & 5 & 5.765 & $1,2,3$ & & & 147.59 & 84 & PO & LBO & 4 & 3.967 & $1,2,3$ \\
\hline 143.46 & $90 \mathrm{TH}$ & L85 & 5 & 0.765 & 1,2 & & & 147.80 & 91 & PA & LB2 & 5 & 0.174 & $1,2,3$ \\
\hline 143.74 & $79 \mathrm{AU}$ & LAI. & 3 & 1.276 & 1,3 & & & 147.80 & 69 & $T M$ & LYo & 3 & 1.290 & 1 \\
\hline 143.74 & $68 \mathrm{ER}$ & $1 Y_{4}$ & 3 & 1.276 & 1,2 & & & 147.80 & 47 & $A G$ & LB4 & 1 & 3.870 & $1,2,3$ \\
\hline 143.74 & $81 \mathrm{TL}$ & LB9 & 4 & 0.957 & 1,2 & & & 147.80 & 12 & HF & 189 & 3 & 1.290 & 1.2 \\
\hline 144.11 & $78 \mathrm{PT}$ & $\lfloor Y \mid$ & 4 & 0.958 & $1,2,3$ & & & 147.80 & 60 & ND & LYS & 2 & 1.935 & 1,2 \\
\hline
\end{tabular}




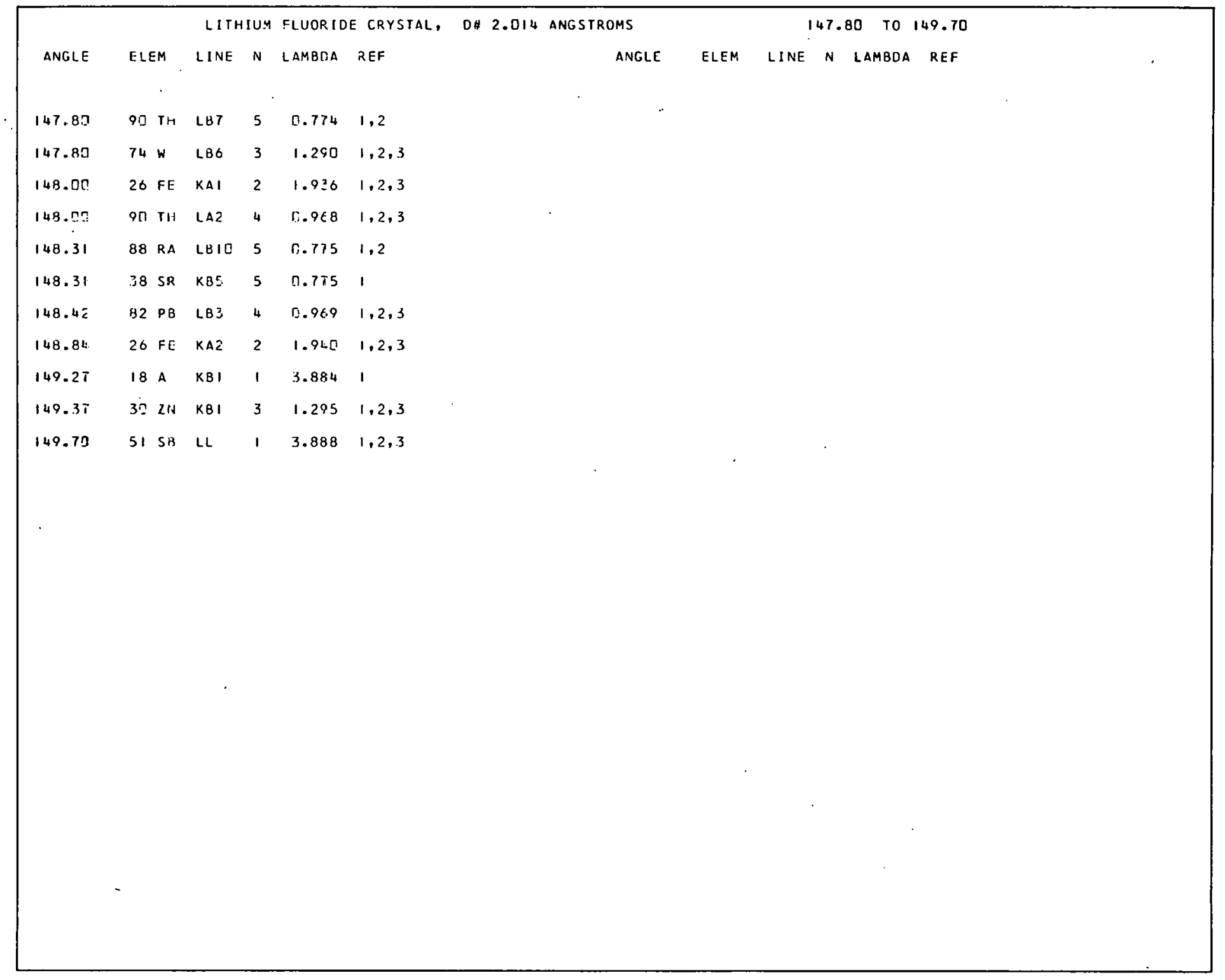

